\title{
Spatial and nonspatial evolutionary games and their applications
}

Citation for published version (APA):

You, L. (2018). Spatial and nonspatial evolutionary games and their applications. [Doctoral Thesis, Maastricht University]. Maastricht University. https://doi.org/10.26481/dis.20180207yl

Document status and date:

Published: 01/01/2018

DOI:

10.26481/dis.20180207yl

Document Version:

Publisher's PDF, also known as Version of record

\section{Please check the document version of this publication:}

- A submitted manuscript is the version of the article upon submission and before peer-review. There can be important differences between the submitted version and the official published version of record.

People interested in the research are advised to contact the author for the final version of the publication, or visit the DOI to the publisher's website.

- The final author version and the galley proof are versions of the publication after peer review.

- The final published version features the final layout of the paper including the volume, issue and page numbers.

Link to publication

\footnotetext{
General rights rights.

- You may freely distribute the URL identifying the publication in the public portal. please follow below link for the End User Agreement:

www.umlib.nl/taverne-license

Take down policy

If you believe that this document breaches copyright please contact us at:

repository@maastrichtuniversity.nl

providing details and we will investigate your claim.
}

Copyright and moral rights for the publications made accessible in the public portal are retained by the authors and/or other copyright owners and it is a condition of accessing publications that users recognise and abide by the legal requirements associated with these

- Users may download and print one copy of any publication from the public portal for the purpose of private study or research.

- You may not further distribute the material or use it for any profit-making activity or commercial gain

If the publication is distributed under the terms of Article $25 \mathrm{fa}$ of the Dutch Copyright Act, indicated by the "Taverne" license above, 


\section{Spatial and Nonspatial Evolutionary Games and Their Applications}

\section{DISSERTATION}

to obtain the degree of Doctor at Maastricht University, on the authority of the Rector Magnificus,

Prof. dr. Rianne M. Letschert, in accordance with the decision of the Board of Deans, to be defended in public on Wednesday 7 February, 2018 at 14:00 hours 


\section{Supervisors:}

Prof. dr. F. Thuijsman

Prof. dr. ir. R.L.M. Peeters

\section{Co-supervisor:}

Dr. K. Staňková

\section{Assessment Committee:}

Prof. dr. G.B. Weiss (chairman)

Prof. dr. A.L.A.J. Dekker

Prof. dr. H.J.M. Peters

Dr. B. Újvári (Deakin University)

Dr. Y. Viossat (Université Paris-Dauphine)

The research reported in this thesis was financially supported by the China Scholarship Council (under the grant No. 201206070054), the European Union's Horizon 2020 research and innovation program (under the Marie Sktodowska-Curie grant agreement No. 690817) and the Department of Data Science and Knowledge Engineering, Maastricht University.

The left picture on the cover is taken from Müller Klieser et al. (2002).

\section{ISBN 978-94-6361-056-8}

(C) Li You, 2018.

All rights reserved. No part of this publication may be reproduced, stored in a retrieval system, or transmitted, in any form or by any means, electronically, mechanically, photocopying, recording or otherwise, without prior permission of the author. 
To my loving parents.

致我摰愛的父母。 

1 Introduction $\quad 1$

1.1 Basics of game theory _. . . . . . . . . . . . . . . . . . 1

1.2 Basics of evolutionary game theory . . . . . . . . . . . . . . 6

1.3 Evolutionary game dynamics . . . . . . . . . . . . . . . . . . . . 9

1.4 Spatial games . . . . . . . . . . . . . . . . . . . . . . 15

1.5 An overview of this thesis . . . . . . . . . . . . 18

1.6 Reading guidelines . . . . . . . . . . . . . . . . . . . . . 21

2 Joining vs. opting out a predator-prey game 23

2.1 Introduction . . . . . . . . . . . . . . . . . . . 25

2.2 The predator-prey game between the predatory mites and the fruit-tree red spider mites . . . . . . . . . . . . . . . . . . . 27

2.2.1 Observations regarding the system of the predatory mites and the fruit-tree red spider mites . . . . . . . . . . . . . . . 27

2.2 .2 The model . . . . . . . . . . . . . . . . . . . 27

2.2.3 Hamilton-Jacobi-Bellman approach to find optimal strategies for the predator and the prey . . . . . . . . . . . . . . . . . 29

2.3 Another way to model the problem . . . . . . . . . . . . . . 39

2.4 Conclusion \& discussion . . . . . . . . . . . . . . . . . . . . 41

3 Discrete-space vs. continuous-space games 45

3.1 Introduction . . . . . . . . . . . . . . . . . . . 47

3.2 The nonspatial game . . . . . . . . . . . . . . . . . . . . . . . . . . . . 49

3.3 Spatial games . . . . . . . . . . . . . . . . . . 50

3.3 .1 Game field . . . . . . . . . . . . . . . . 50

3.3 .2 Spatial game rules . . . . . . . . . . . . . . . . . 51

3.4 Case studies . . . . . . . . . . . . . . . . . . . . . . . 52 
3.4 .1 The generalized RSP game . . . . . . . . . . . . . . . . . . 52

3.4.2 Enhanced RSP game . . . . . . . . . . . . . . . 53

3.4.3 Settings of the case studies . . . . . . . . . . . . . . . 54

3.4.4 Case study 1: Impact of the dispersal conflict . . . . . . . . . . 57

3.4.5 Case study 2: Impact of the interaction radius . . . . . . . . . . . 61

3.4.6 Case study 3: Impact of the lifespan . . . . . . . . . . . . . . 65

3.5 Conclusion \& discussion . . . . . . . . . . . . . . . . . . . . . 67

4 A game-theoretical approach to microbial coexistence $\quad 71$

4.1 Introduction . . . . . . . . . . . . . . . . . . . . . . . . . 73

4.2 The game model . . . . . . . . . . . . . . . . . . . . . 75

4.2.1 The basic model . . . . . . . . . . . . . . 76

4.2 .2 Quorum sensing model . . . . . . . . . . . . . . . . . 77

4.3 Case studies on coexistence . . . . . . . . . . . . . . . . . . . 78

4.3.1 Case study 1: Impact of the interaction matrix density . . . . . . . . 79

4.3.2 Case study 2: The number of neighbors . . . . . . . . . . . . . . . 79

4.3.3 Case study 3: Basic model vs. quorum sensing model . . . . . . . . . 81

4.4 Conclusion \& discussion . . . . . . . . . . . . . . . . . . . . . . . 81

$\begin{array}{lll}5 & \text { Spatial and nonspatial games of tumorigenesis } & 87\end{array}$

5.1 Introduction . . . . . . . . . . . . . . . . . . . . . . . . 89

5.2 Models: Replicator dynamics and its spatial variant . . . . . . . . . . . . 91

5.2.1 Background: Metastatic castrate-resistant prostate cancer . . . . . . 91

5.2 .2 Model basics . . . . . . . . . . . . . . . . . . 92

5.2.3 Replicator dynamics in metastatic castrate-resistant prostate cancer . $\quad 92$

5.2.4 Spatial replicator dynamics in metastatic castrate-resistant prostate cancer . . . . . . . . . . . . . . . . . . 94

5.3 Tumor growth and composition . . . . . . . . . . . . . . 96

5.3.1 The effects of the mortality regime on tumor growth . . . . . . . . 98

5.3.2 Spatial vs. nonspatial dynamics . . . . . . . . . . . . . . 102

5.4 Effects of the frequency-dependence radius, the dispersal radius and the densitydependence radius on spatial equilibria . . . . . . . . . . . . . . . . . 105

5.4 .1 Effect of the frequency-dependence radius . . . . . . . . . . 105

5.4 .2 Effect of the dispersal radius . . . . . . . . . . . . . 106

5.4 .3 Effect of the density-dependence radius . . . . . . . . . . . . . . 106

5.5 Conclusion \& discussion . . . . . . . . . . . . . . . . . . . 108

6 Conclusions $\quad 115$

6.1 Answers to research questions . . . . . . . . . . . . . . . . . . 115

6.2 Extensions and limitations . . . . . . . . . . . . . . . . . 118

6.3 Research avenues more far ahead . . . . . . . . . . . . . . . . 120 
$\begin{array}{ll}\text { A Supplement of Chapter } 1 & 143\end{array}$

B Supplement of Chapter $3 \quad 149$

B.1 Results with respect to varying radii . . . . . . . . . . . . . . . . . . 149

B.2 Results with respect to increased lifespan . . . . . . . . . . . . . . . . 149

C Supplement of Chapter $5 \quad 153$

C.1 The nonspatial model . . . . . . . . . . . . . . . . . . 153

C.2 Sensitivity analysis . . . . . . . . . . . . . . . . . 155

C.3 Tables of transient and saturated equilibria . . . . . . . . . . . . 155

C.4 Effects of zero diagonal . . . . . . . . . . . . . . . . . . . 155

$\begin{array}{ll}\text { Acknowledgements } & 165\end{array}$

$\begin{array}{lr}\text { About the author } & 167\end{array}$ 


\section{Introduction}

This thesis focuses on various spatial and nonspatial game-theoretical models of biological interactions. On the one hand, we are interested in general properties of these models, analyze their eco-evolutionary (population and strategy) dynamics and study the factors that impact these dynamics, and, on the other hand, we apply these models to understand interactions between the predatory mites and the fruit-tree red spider mites, to explain coexistence of bacterial strains, and to model tumorigenesis. For each of these biological problems we use a different game-theoretical model, one which we find most suitable for the problem at hand. We therefore start with an introduction to the basics of game theory.

\subsection{Basics of game theory}

Game theory provides analytic tools to study decision making in situations where the outcome of one's decision may depend not just on one's own choices, but also on the decisions made by others. Such situations, which we will call games, arise in many different contexts. Not only games like cards, chess or sports, but also situations which we do not usually call games, can be modeled and analyzed using game theory. Examples include the pricing of a new product when other firms have similar new products, choosing a route through a transportation network, deciding whether to adopt an aggressive or passive stance in international relations or deciding how to negotiate a job contract.

Game theory was established as a field since the birth of the book "Theory of games and economic behavior" by the mathematician John von Neumann and the economist Oskar Morgenstern (Von Neumann and Morgenstern, 1944). Since the 1950's, game-theoretical 
models have been used in social sciences and economics. From the 1960's onwards, game theory has been also used to describe and understand many different phenomena in biology. To understand some basic notions of game theory, let us start with an example.

Game 1 Suppose two animals, Animal 1 and Animal 2, are engaged in a contest in which they will decide how to divide a piece of food between them. Each animal can choose to act aggressively or friendly and both animals act simultaneously. If both of them act aggressively, they end up being injured and the food is destroyed, thus neither animal gets the food. If both animals act friendly, they share the food evenly. If one animal behaves aggressively while the other one behaves friendly, the aggressive animal obtains most of the food while the friendly one gets the rest. We use numbers to indicate what portion of food an animal can get in this game: $0, \frac{1}{6}, \frac{1}{2}$ and $\frac{5}{6}$ represent the outcomes as getting no food, a minor piece, a half and a major piece of the food, respectively. We represent possible outcomes of the game in Table 1.1, where Animal 1 chooses a row and gets outcomes highlighted in blue and Animal 2 chooses a column and gets outcomes highlighted in red. Clearly, in this contest,

\begin{tabular}{|c|c|c|c|}
\hline \multirow[b]{2}{*}{ Animal 1} & & $\begin{array}{r}\text { Anim } \\
\text { act aggressively }\end{array}$ & 2 \\
\hline & $\begin{array}{l}\text { act aggressively } \\
\text { act friendly }\end{array}$ & $\begin{array}{l}0,0 \\
\frac{1}{6}, \frac{5}{6}\end{array}$ & $\begin{array}{l}\frac{5}{6}, \frac{1}{6} \\
\frac{1}{2}, \frac{1}{2}\end{array}$ \\
\hline
\end{tabular}

Table 1.1: Game 1

the portion of food an animal gets does not depend on this animal's choice only, but also on what the other one decides to do. Therefore, analyzing what both animals are likely to do is necessary for predicting the outcome of this game. The process of considering possible outcomes by taking into account the decisions made by all players (Animal 1 and Animal 2 in Game 1) is a standard procedure of game theory. Let us now introduce the basic elements of a game:

\section{Basic elements of a game}

(i) players - the participants in the game. In Game 1, the two animals are the two players;

(ii) strategies (actions) - the decisions that players take. In Game 1, there are two strategies: "act aggressively" and "act friendly". We denote the strategies by $H$ and $D$, respectively, as Game 1 is an example of is the classical Hawk-Dove game introduced by Maynard Smith (1974), where Hawk and Dove strategies refer to the aggressive and friendly behaviors, respectively;

(iii) payoffs - players' outcomes that may depend on the strategies chosen by other players as well. Each player wants to maximize its ${ }^{1}$ own payoff. In Game 1, the payoffs are defined by the matrix in Table 1.1. We see that the payoffs of the row and column

\footnotetext{
${ }^{1}$ Throughout this thesis we assume players are gender-neutral.
} 
players are symmetric in Table 1.1. Therefore, the game can be fully described by listing the payoffs for the row player only, see Table 1.2. Such games are called symmetric games.

\begin{tabular}{c|cc} 
& $H$ & $D$ \\
\hline$H$ & 0 & $\frac{5}{6}$ \\
$D$ & $\frac{1}{6}$ & $\frac{1}{2}$
\end{tabular}

Table 1.2: The payoff matrix for Game 1

Game 1 is a game with two players and two strategies. The strategies are denoted by $H$ and $D$ and each player has to choose one of them, simultaneously and independently of the other player's choice. Here we assume that Game 1 is played only once. Moreover, we assume that each player is rational and has full knowledge of possible strategies and corresponding outcomes for all players. In general the number of players, as well as the number of strategies, may of course be larger or smaller than 2 .

Best reply and dominant strategy It is easier to decide what to do if one knows, or can predict, what strategies the other players are going to play. In Game 1, if Animal 2 plays $H$, then Animal 1 should play $D$; if Animal 2 plays $D$, then Animal 1 should play $H$, as these choices give Animal 1 the highest payoff when the strategy of Animal 2 is fixed. We observe that $H$ is the best reply to $D$ and $D$ is the best reply to $H$.

Generally, in a game, each player wants its strategy to be a best reply to the strategies chosen by all other players. Imagine a game with $m$ players, where each player can choose a strategy from a set of strategies $S=\{1, \ldots, n\}$. For any player $k \in\{1,2, \ldots m\}$, let $s_{k} \in S$ denote its strategy and $\left(s_{1}, \ldots, s_{m}\right)$ the strategy profile (an $m$-tuple of strategies) for all $m$ players. Let $s_{-k}=\left(s_{1}, \ldots, s_{k-1}, s_{k+1}, \ldots, s_{m}\right)$ denote the strategy profile chosen by all players except for player $k$. From player $k$ 's point of view, $s_{-k}$ is the strategy being played against its own strategy $s_{k}$. Let us denote by $w_{k}\left(s_{k}, s_{-k}\right)$ the payoff that the player $k$ receives when playing $s_{k}$, while the other players are jointly playing $s_{-k}$. We say $s_{k}$ is a best reply to $s_{-k}$, if

$$
w_{k}\left(s_{k}, s_{-k}\right) \geq w_{k}\left(s_{k}^{\prime}, s_{-k}\right), \text { for all } s_{k}^{\prime} \in S
$$

In Game $1, H$ is a best reply to $D$ and $D$ is a best reply to $H$.

However, even if the players are aware of their best replies, Animal 1 may not always be able to choose a best reply to a strategy of Animal 2, since the game is played simultaneously and independently, and it may well be unclear to Animal 1 which strategy Animal 2 will play, for example if there are more of such best strategies. For Animal 1, there is no single strategy that is a best reply to all strategies of Animal 2. This brings us to the concept of strategic dominance, which can be defined in terms of the best reply: We say that a strategy is a so-called dominant strategy, if this strategy is always a best reply to any strategy profile of the other players. In Game 1, neither Animal 1 nor Animal 2 has a dominant strategy. 
Nash equilibrium If for every player $k \in\{1,2, \ldots, m\}$, its strategy $s_{k}$ is a best reply to $s_{-k}$, then the strategy profile $\left(s_{1}, \ldots, s_{m}\right)$ forms a Nash equilibrium, an equilibrium state in which no player can be better off (i.e., getting a higher payoff) by unilaterally deviating from its current strategy. The Nash equilibrium is named after John Nash, who introduced this concept (Nash, 1950). There are two Nash equilibria in Game 1: (1) Animal 1 plays $H$ while Animal 2 plays $D,(2)$ Animal 1 plays $D$ while Animal 2 plays $H$. For brevity, we denote these two Nash equilibria by $(H, D)$ and $(D, H)$, respectively.

Mixed strategy In Game 1 the two Nash equilibria yield different payoffs. It is therefore difficult to predict which of these will be played. While there are other games with multiple Nash equilibria, such as Coordination games (Cooper et al., 1990), there are also games which have no Nash equilibria if each player $k$ has to select a unique strategy $s_{k}$ from the finite set of actions $S$, as for example the Rock-Scissors-Paper game. The game of choosing sides is an example of a coordination game. ${ }^{2}$ Assume two drivers approach each other on a narrow road, and both have to swerve in order to avoid a head-on collision. If both choose the same swerving strategy, Left or Right, they will manage to pass each other, otherwise they will collide. In the payoff matrix depicted in Table 1.3, a successful passing and a collision are represented by payoffs of 10 and 0 , respectively. The Rock-Scissors-Paper game is usually played between two players, in which the players simultaneously and independently, chooses one of the three strategies Rock, Scissors or Paper. The game has the rule "Rock crushes Scissors", "Scissors cuts Paper" and "Paper covers Rock". If both players choose the same strategy, the game is a tie. In the payoff matrix shown in Table 1.4, the outcomes of winning, losing and tying are represented by payoffs of $1,-1$ and 0 , respectively. From this payoff

\begin{tabular}{l|cc} 
& Left & Right \\
\hline Left & 10 & 0 \\
Right & 0 & 10
\end{tabular}

Table 1.3: The payoff matrix for the game of choosing sides

\begin{tabular}{l|ccc} 
& Rock & Scissors & Paper \\
\hline Rock & 0 & 1 & -1 \\
Scissors & -1 & 0 & 1 \\
Paper & 1 & -1 & 0
\end{tabular}

Table 1.4: The payoff matrix for the Rock-Scissors-Paper game

matrix one can see that none of the entries of the Rock-Scissors-Paper game corresponds to a Nash equilibrium. However, if player 2 would randomize over its actions by playing each with probability $\frac{1}{3}$, then the expected payoff to player 1 would be 0 for each of the actions Rock, Scissors and Paper. This means that each of these actions would be a best

\footnotetext{
${ }^{2}$ The game of choosing sides is a coordination game, because the two players' goal is to coordinate on the same strategy.
} 
reply to this particular randomization by player 2 . That is how we arrive at the concept of a mixed strategy, which applies when players are allowed to randomize over their actions. A mixed strategy for a player is simply a probability distribution over the set of all possible strategies for this player. So, each player $k \in\{1, \ldots, m\}$ playing a mixed strategy assigns a probability $p_{k}(l) \geq 0$ to each of its possible strategies $l \in S$, where $\sum_{l=1}^{n} p_{k}(l)=1$. We call $p_{k}=\left(p_{k}(1), \ldots, p_{k}(n)\right)$ a mixed strategy for player $k$. To distinguish the definition of a mixed strategy from the concept of strategy introduced earlier, we call every $l \in S$ a pure strategy. Note that a pure strategy is a trivial case of a mixed strategy. In Game $1, S=\{H, D\}$ is the set of two pure strategies, and $\left(p_{k}(H), 1-p_{k}(H)\right)$ is a mixed strategy for player $k \in\{1,2\}$ playing $H$ with probability $p_{k}(H) \in[0,1]$ and $D$ with probability $1-p_{k}(H)$.

If players use mixed strategies, they do so to maximize their expected payoffs. In Game 1, assuming that Animal 2 plays the mixed strategy $\left(p_{2}(H), 1-p_{2}(H)\right)$, Animal 1 would expect the payoff

$$
w_{H}=p_{2}(H) a_{11}+\left(1-p_{2}(H)\right) a_{12}
$$

if it plays $H$, and

$$
w_{D}=p_{2}(H) a_{21}+\left(1-p_{2}(H)\right) a_{22}
$$

if it plays $D$, where $A=\left(a_{i j}\right)$ denotes the payoff matrix in Table 1.2, and $a_{i j}$ denotes its entry at the $i$-th row and the $j$-th column $(i, j \in\{1,2\})$. Animal 1's expected payoff $w_{H}$ ( or $w_{D}$ ) is linear in Animal 2's probability distribution $\left(p_{2}(H), 1-p_{2}(H)\right.$ ) over the set of strategies $S=\{H, D\}$ and the situation is the same from Animal 2's point of view. In short we can say that the payoffs to players are linear on the other player's strategy space.

Mixed-strategy Nash equilibrium Let us continue with Game 1. If $w_{H}$ is greater than $w_{D}$, Animal 1 should play $H$; if $w_{H}$ is smaller than $w_{D}$, Animal 1 should play $D$. In either case, Animal 1 should play a pure strategy as the best reply to Animal 2's strategy $\left(p_{2}(H)\right.$, $\left.1-p_{2}(H)\right)$. Therefore, when seeking for a mixed-strategy Nash equilibrium for this particular game, we are looking for a pair of strategies for which the expected payoffs should satisfy

$$
w_{H}=w_{D}
$$

i.e.,

$$
p_{2}(H) \cdot 0+\left(1-p_{2}(H)\right) \cdot \frac{5}{6}=p_{2}(H) \cdot \frac{1}{6}+\left(1-p_{2}(H)\right) \cdot \frac{1}{2} .
$$

This equation is satisfied for $p_{2}(H)=\frac{2}{3}$. Since the game is symmetric, the situation is the same from Animal 2's point of view. Thus, the pair $\left(\left(\frac{2}{3}, \frac{1}{3}\right),\left(\frac{2}{3}, \frac{1}{3}\right)\right)$ is the unique mixedstrategy Nash equilibrium in Game 1, while the two Nash equilibria $(H, D)$ and $(D, H)$, can be referred to as the pure-strategy Nash equilibria. In his seminal paper, John Nash proved that a mixed-strategy Nash equilibrium exists in any $m$-person game in which all players have finitely many pure actions (Nash, 1950).

Interpretation of the mixed-strategy Nash equilibrium When we talk about a mixed-strategy Nash equilibrium we are referring to a Nash equilibrium where at least one 
player is playing a mixed strategy. Having derived the mixed-strategy Nash equilibrium for Game 1, it is useful to think of its interpretation. If Game 1 is played only once, the mixed strategy means that an animal plays $H$ or $D$ with a certain probability.

Another interpretation of the mixed-strategy applies when a game is repeated many times: The mixed strategy then indicates how many times from the total number of repetitions each strategy is supposed to be played. Suppose Game 1 is played repeatedly between Animal 1 and Animal 2, where in each repetition both animals play $\left(\frac{2}{3}, \frac{1}{3}\right)$ according to the mixed-strategy Nash equilibrium. Then, on average each animal will actually choose $H \frac{2}{3}$ of the time and $D \frac{1}{3}$ of the time. On average each animal would get $\frac{5}{18}$, while in each of the pure strategy Nash equilibria one of them would get less.

Especially in biological applications of game theory, we are often focusing on populations of individuals, which have fixed strategies that they cannot change. Then, the pure actions are often interpreted as different types of individuals and a mixed strategy as a population distribution over these types. In such problems, we are often interested in equilibrium distributions over the types in the population. The part of game theory investigating such problems is called Evolutionary Game Theory and we will introduce its basics below.

\subsection{Basics of evolutionary game theory}

One of the first and most influential works which made a formal connection between game theory and biology was written by J. Maynard Smith and G. R. Price, who provided considerable insights on behavioral strategies in animal conflicts (Maynard Smith and Price, 1973) and established the domain of Evolutionary Game Theory. Evolutionary game theory applies game-theoretical analysis to study evolution, where games are often played within a population.

Before we proceed to a population version of evolutionary games, let's recall Game 1 and give more thoughts about the payoffs: Suppose that in Game 1 food as a payoff transforms into nutrients, and, therefore, the animal that obtains a larger piece of food will become more fit (assuming both animals process nutrients equally effectively). Bearing this in mind, let us introduce Game 2.

Game 2 Consider a population composed of many animals. Any two of these animals may engage in a pairwise interaction as described in Game 1. Here the individuals do not make explicit decisions, i.e., they do not ask themselves "How do I obtain a piece of food, do I go and fight for it or shall I share with the other?" We rather assume that each animal is hardwired to play one particular strategy throughout its entire life. We now interpret the strategies $H$ and $D$ as different types (such as phenotypes or genotypes). The only way how the population frequencies of these types can evolve over time is via reproduction, where the types $H$ and $D$ are passed onto the offspring. In this game we relate reproduction to interactions of two individuals (i.e., one as a row player and the other a column player). While these interactions directly lead to payoffs determining how fit the row player is, we further assume a fitter individual tends to have a higher rate of reproduction (where its 
offspring is assumed to be of its type). Hence over time a fitter type will likely produce more offspring, causing the frequency of its type in the population to increase.

Game 2 is an example of a population game. We will now introduce the population games in a more formal way.

Population game Suppose $m$ individuals of $n$ distinct types (where $m$ is much greater than $n$, i.e., $m \gg n$ ) participate in a game. The $n$ types are distributed in the population as $^{3} x=\left(x_{1}, x_{2}, \ldots, x_{n}\right) \in \mathbb{R}^{n}$, where $x_{i} \geq 0$ is the proportion or frequency of type $i$ for each $i \in\{1, \ldots, n\}$, and where $\sum_{i=1}^{n} x_{i}=1$. The elements of this game, i.e., players, strategies and payoffs, require different interpretations:

(i) A player can be an individual from the population, e.g., player $k \in\{1,2, \ldots, m\}$; players of the same type $i$ are usually represented by their proportion $x_{i}$, while an entire population is usually represented by its type distribution $x .{ }^{4}$

(ii) Payoffs, termed fitnesses, have a biological meaning. Fitness translates into reproductive success (as described in Game 2) such as the number of offspring or the rate of reproduction (Godfrey Smith, 2009; Zietsch et al., 2014). For a population, its fitness is the expected payoff when it plays against itself. Here the underlying assumption is that for each individual in the population its reproductive success depends on a random interaction with another individual of the same population. For a particular type from a population, its fitness is the expected payoff it receives when playing against an arbitrary type from the population. Within evolutionary game theory, the payoff matrix is often called the fitness matrix.

Population games generally describe situations in which players of distinct types interact and these interactions will determine for each type its success over time, i.e., its ability to persist in the population. The types receiving higher fitness are more successful. But will these types be successful over a long period of time or will they be beaten by another type at some point? This leads to the notion of the Evolutionarily Stable Strategy (ESS), which defines a stable population distribution that would persist once prevalent. The concept of Evolutionarily Stable Strategy (ESS) was introduced by Maynard Smith and Price (1973). We introduce this concept more formally.

Evolutionarily Stable Strategy An ESS is a strategy that, when adopted by an entire population, is immune against invasion by a small number of mutants, who come from the same entire population but use a different strategy. This implies that (an average member of) the mutants should have a lower fitness than (an average member of) the non-mutant

\footnotetext{
${ }^{3}$ In this thesis we write the population distribution, e.g., $x$, as a row vector.

${ }^{4}$ Population models consider proportions as players, assuming populations are well mixed. Population models date back to the 19th century, e.g., Richards (1959), and in the past years these models have been used in evolutionary game theory (Maynard Smith and Price, 1973). Population models do not track information of each individual, but that of an average individual.
} 
population. Since fitness translates into reproductive success, its lower fitness would cause the mutant population to shrink over time and die out.

To formulate ESS, let us consider a population game where $n$ types are distributed according to a population distribution $x$ and where $A$ is the fitness matrix. If in this population $x$ a small proportion $\mu>0$ is replaced by mutants that are distributed as $\hat{x}$ (represented as a row vector over the same $n$ types), then the new population distribution will be given by

$$
x_{\mu \hat{x}}=(1-\mu) x+\mu \hat{x} .
$$

Interpreting the population distributions as strategies, this means that $x$ is an ESS if for any small $\mu>0$ and any mutant $\hat{x}$, strategy $x$ always yields a strictly higher fitness against the strategy $x_{\mu \hat{x}}$ than the mutant strategy $\hat{x}$ does, i.e.,

$$
x A x_{\mu \hat{x}}^{\top}>\hat{x} A x_{\mu \hat{x}}^{\top} .
$$

This can be rewritten as

$$
(1-\mu)\left(x A x^{\top}-\hat{x} A x^{\top}\right)+\mu\left(x A \hat{x}^{\top}-\hat{x} A \hat{x}^{\top}\right)>0 .
$$

Therefore, $x$ is an ESS if and only if the following conditions are both satisfied:

(i) $x A x^{\top} \geq \hat{x} A x^{\top}$ for all $\hat{x}$;

(ii) if $\hat{x} \neq x$ and $x A x^{\top}=\hat{x} A x^{\top}$, then $x A \hat{x}^{\top}>\hat{x} A \hat{x}^{\top}$.

According to (i), $x$ should be a best reply to itself, implying that $(x, x)$ is a Nash equilibrium. According to (ii), if a different strategy $\hat{x}$ is also a best reply to $x$, then $x$ should perform strictly better against $\hat{x}$ than $\hat{x}$ does against itself. This condition prevents the population strategy from drifting away from $x$, and we consider this a condition for evolutionary stability. It is well possible to have a game where $(x, x)$ is a Nash equilibrium, while $x$ is not an ESS because the second condition is violated. While each game always has at least one (possibly mixed-strategy) Nash equilibrium (Nash, 1950), some symmetric games may have no ESS, while others may have more than one (Maynard Smith, 1974).

Let's now re-examine Game 2. Assume the population is distributed as $x=\left(x_{H}, 1-x_{H}\right)$ where $x_{H}$ denotes the proportion of the type $H$ in the population. Recall that this game has two pure-strategy Nash equilibria: $(H, D)$ and $(D, H)$, and one mixed-strategy Nash equilibrium where both players choose $H$ with probability $\frac{2}{3}$.

The fact that $H$ is not a best reply to itself and $D$ is not a best reply to itself, indicates that a population consisting of only $H$-individuals would be invaded by a small number of $D$ mutants, and vice versa. Thus, neither pure strategy of the population (or population strategy) $x=(1,0)$ nor $x=(0,1)$ is an ESS. It remains to be analyzed whether or not $x=\left(\frac{2}{3}, \frac{1}{3}\right)$ is an ESS.

We observe that, for any strategy $\hat{x}=\left(\hat{x}_{H}, 1-\hat{x}_{H}\right)$, where $\hat{x}_{H}$ is the proportion of type 
$H$, when playing against $x=\left(\frac{2}{3}, \frac{1}{3}\right)$, the payoff is always $5 / 18$ :

$$
\begin{aligned}
\hat{x} A x^{\top} & =\left(\hat{x}_{H}, 1-\hat{x}_{H}\right)\left(\begin{array}{cc}
0 & \frac{5}{6} \\
\frac{1}{6} & \frac{1}{2}
\end{array}\right)\left(\begin{array}{c}
\frac{2}{3} \\
\frac{1}{3}
\end{array}\right) \\
& =\left(\hat{x}_{H}, 1-\hat{x}_{H}\right)\left(\begin{array}{c}
\frac{5}{18} \\
\frac{5}{18}
\end{array}\right) \\
& =\frac{5}{18}
\end{aligned}
$$

Because $\hat{x} A x^{\top}=x A x^{\top}$ for any $\hat{x}$, we have to examine the second condition for $x=\left(\frac{2}{3}, \frac{1}{3}\right)$ to be an ESS, i.e. we have to show that $x A \hat{x}^{\top}>\hat{x} A \hat{x}^{\top}$ for all $\hat{x} \neq x$. This follows from

$$
\begin{aligned}
x A \hat{x}^{\top} & =\left(\frac{2}{3}, \frac{1}{3}\right)\left(\begin{array}{cc}
0 & \frac{5}{6} \\
\frac{1}{6} & \frac{1}{2}
\end{array}\right)\left(\begin{array}{c}
\hat{x}_{H} \\
1-\hat{x}_{H}
\end{array}\right) \\
& =\frac{13}{18}-\frac{2}{3} \hat{x}_{H},
\end{aligned}
$$

and

$$
\begin{aligned}
\hat{x} A \hat{x}^{\top} & =\left(\hat{x}_{H}, 1-\hat{x}_{H}\right)\left(\begin{array}{cc}
0 & \frac{5}{6} \\
\frac{1}{6} & \frac{1}{2}
\end{array}\right)\left(\begin{array}{c}
\hat{x}_{H} \\
1-\hat{x}_{H}
\end{array}\right) \\
& =-\frac{1}{2} \hat{x}_{H}^{2}+\frac{1}{2} .
\end{aligned}
$$

Therefore,

$$
\begin{aligned}
x A \hat{x}^{\top}-\hat{x} A \hat{x}^{\top} & =\frac{13}{18}-\frac{2}{3} \hat{x}_{H}+\frac{1}{2} \hat{x}_{H}^{2}-\frac{1}{2} \\
& =\frac{1}{18}\left(3 \hat{x}_{H}-2\right)^{2},
\end{aligned}
$$

and hence $x A \hat{x}^{\top}>\hat{x} A \hat{x}^{\top}$ for all $\hat{x}_{H} \neq \frac{2}{3}$, implying that $x=\left(\frac{2}{3}, \frac{1}{3}\right)$ is an ESS of Game 2 .

While the second condition of the ESS definition relates to the dynamic stability of the population, it does not contain any specification of an actual dynamic process. In the following section we will therefore address the evolutionary game dynamics.

\subsection{Evolutionary game dynamics}

Evolutionary dynamics are very helpful in understanding biological processes. For example, Pfeiffer et al. (2001) did a pioneering work which used game-theoretical approaches in biochemistry. They analyzed two different strategies, respiration and fermentation, in ATPproducing pathways through a game-theoretical model, and they gave insights into why many micro-organisms may rely on fermentation even under aerobic conditions. Dingli et al. (2009) studied interactions between different types of cancer cells and stromal cells in the bone in multiple myeloma. Their dynamical model showed that, targeting the interactions between the tumor and the stromal cells so that those stromal cells can effectively outcompete the tumor, is a promising approach, while traditionally the cure of myeloma is targeting 
directly on the cancer cells. Outside biology there are other scientific fields that make use of evolutionary dynamics. For example, in multiagent systems ${ }^{5}$ decision making may be modeled and evaluated by evolutionary dynamics (Parsons and Wooldridge, 2002).

Before we move to standard forms of evolutionary game dynamics, we will introduce the basics of dynamical systems and stability analysis.

\section{Dynamical systems}

Dynamical systems are used to mathematically describe the time evolution of a process under study, taking all the relevant variables, their interactions and inter-dependencies into account. In the literature, e.g. Olsder (1997), there are many different system classes and representations in use; here we will restrict ourselves to some of the simplest ones that are yet rich enough and sufficiently powerful for the purposes of this thesis.

For dynamics taking place in continuous time, we focus on systems characterized by a system of first-order ordinary differential equations (ODEs):

$$
\dot{x}=f(x) .
$$

The time set $\Gamma$ involved, is an interval (with non-empty interior) in $\mathbb{R}$. We typically choose it to be $\Gamma=[0, \infty)$ which enables us to consider systems starting from some initial state at time $t=0$, for which (among other things) we can study what happens when time goes to infinity. At each time instant $t \in \Gamma$, the state vector $x(t)=\left(x_{1}(t), x_{2}(t), \ldots, x_{n}(t)\right)$ is a point in the state space (also called the phase space) $X \subseteq \mathbb{R}^{n}$. Without going into full details, this space $X$ is assumed to be suitably selected: to be connected and to have a non-empty interior, and such that solutions of the system of ODEs starting in $X$ do not leave $X$. The dynamical map $f: X \rightarrow \mathbb{R}^{n}$ which defines the system is required to be sufficiently smooth for the system of ODEs to have a unique local solution for every initial point $x(0) \in X$. ${ }^{6}$

A state trajectory is defined as the collection of points visited by a solution of the system of ODEs (1.1), starting at some initial point $x_{0}$. It has the form $\zeta\left(x_{0}\right)=\{x(t) \in X \mid x(0)=$ $\left.x_{0}, t \in \Gamma\right\}$, for a choice of initial state $x_{0} \in X$. An equilibrium point $x^{*} \in X$ of the dynamical system (1.1) is a point which satisfies $f\left(x^{*}\right)=0$; but to avoid confusion with game-theoretic equilibria we will rather call this a steady point. Note that steady points can be identified with state trajectories consisting of a single point (i.e., "singleton trajectories"), because solutions to the ODE were assumed to be locally unique. For steady points and state trajectories of

\footnotetext{
${ }^{5}$ In a multiagent system, agents are autonomous entities placed within an environment. These agents perceive through sensors and act upon their environment through actuators, towards achieving some given goal (Bloembergen, 2015).

${ }^{6}$ Because in this set-up $f$ is a function of $x$ alone, it only depends on time implicitly (i.e., through $x(t)$ ); then the system is either called autonomous or time-invariant, depending on what is common in the specific field of research. It implies that if a solution reaches a certain state, from there on it proceeds in one and the same way regardless of its history and of the time at which that state was reached. This allows one to visualize the system dynamics by plotting non-intersecting state trajectories in the state space (phase space). Generalizations of this set-up allow $f$ to depend also on time $t$, or on player strategies (c.q. control inputs) $u$, or both. Other extensions involve the incorporation of time-delays or stochastic terms in the equation. We will not go into this here.
} 
dynamical systems, many different concepts of stability exist (Olsder, 1997). In this thesis we focus on the most common ones only.

A steady point $x^{*}$ is called stable, if for every open neighborhood $U$ of $x^{*}$ in $X$ there exists another open neighborhood $V$ of $x^{*}$ in $X$ such that for all $x_{0} \in V$ the state trajectory $\zeta\left(x_{0}\right)$ is entirely contained in $U$. A steady point $x^{*}$ is called asymptotically stable if in addition all solutions $x(t)$ starting in such $V$ converge to $x^{*}$ for $t$ tending to infinity. If a steady point is stable but not asymptotically stable, it is called neutrally stable. If it is not stable, it is called unstable.

For a state trajectory $\zeta\left(x_{0}\right)$, one can introduce stability concepts in a similar way, by considering neighborhoods of trajectories. If a state trajectory corresponds to a periodic solution $x(t)$ of the dynamical system, one speaks of a cycle. By extension, a state trajectory (e.g., a steady point, a cycle, or any other trajectory) is called stable if all trajectories starting in a sufficiently small neighborhood of $\zeta\left(x_{0}\right)$ remain close to $\zeta\left(x_{0}\right)$ according to some appropriate distance measure. And, it is called asymptotically stable if in addition these trajectories all converge to $\zeta\left(x_{0}\right)$ as time goes to infinity. Other useful stability concepts in Bronshtein et al. (2015) can be more restrictive, e.g., by requiring convergence to occur uniformly over time, but we will not consider them in this thesis.

An asymptotically stable steady point (state trajectory) of a dynamical system (1.1) is also called an attractor. For an unstable steady point (state trajectory), one speaks of a repeller (also known as repellor, see for example Akin (2010); Osipenko (2007); Arrowsmith and Place (1992)) if it turns into an attractor when time is reversed. In that case there exists a neighborhood $U$ (of the steady point c.q. state trajectory) such that for all initial states in $U$ except for the steady point (points on the state trajectory), the resulting trajectory leaves $U$ at some moment in time. Finally, a steady point $x^{*}$ in this thesis is called a saddle point if it is unstable and every neighborhood of $x^{*}$ contains a point $x_{0} \neq x^{*}$ for which the trajectory converges to $x^{*}$ when time goes to infinity.

If the state space is the entire Euclidean space $X=\mathbb{R}^{n}$ and $f: x \mapsto x M$ is a linear map with an $n \times n$ real matrix $M$, we speak of a linear dynamical system. For such systems, the eigenvalue and eigenspace structure of $M$ is well-known to fully determine the nature of the steady point at the origin, $x^{*}=0$. Asymptotic stability holds, and $x^{*}=0$ is an attractor, if and only if all eigenvalues of $M$ are in the open left half of the complex plane (i.e., in $\{z \in \mathbb{C} \mid \operatorname{Re}(z)<0\})$. Stability holds if and only if all eigenvalues of $M$ are in the closed left half of the complex plane while those which are on the imaginary axis (if any) have Jordan blocks of size $1 \times 1$ only (Olsder, 1997). The steady point $x^{*}=0$ is a repeller if and only if all eigenvalues of $M$ are in the open right half of the complex plane.

A non-linear dynamical system is usually approximated by linear equations (linearization): Suppose the smooth dynamical system (1.1) is non-linear, let the Jacobian matrix of (1.1) be an $n \times n$ matrix $J(x)=\left(J_{i j}\right)$ where

$$
J_{i j}=\frac{\partial f_{i}}{\partial x_{j}}, \text { for } i, j \in\{1, \ldots, n\} .
$$

Suppose $x^{*}$ is a steady point of the dynamical system (1.1). We denote by $J\left(x^{*}\right)=\left(J_{i j}^{*}\right)$ the Jacobian matrix of (1.1) evaluated at $x^{*}$. Linearizing the dynamical system (1.1) at $x^{*}$, 
using the first-order Taylor approximation and disregarding the errors of this approximation (Kline, 1990), we get the approximation:

$$
\begin{aligned}
\dot{x}_{1} & \approx J_{11}^{*} x_{1}+J_{12}^{*} x_{2}+\cdots+J_{1 n}^{*} x_{n}, \\
\dot{x}_{2} & \approx J_{21}^{*} x_{1}+J_{22}^{*} x_{2}+\cdots+J_{2 n}^{*} x_{n}, \\
& \vdots \\
\dot{x}_{n} & \approx J_{n 1}^{*} x_{1}+J_{n 2}^{*} x_{2}+\cdots+J_{n n}^{*} x_{n},
\end{aligned}
$$

i.e.,

$$
\dot{x} \approx J\left(x^{*}\right) x^{\top}, \text { for } x \in \mathbb{R}^{n} .
$$

When all eigenvalues of $J\left(x^{*}\right)$ have non-zero real parts, the non-linear system (1.1), close to the steady point $x^{*}$, is similar and behaves in the same way as the linear system (1.2) (for which we are able to find solutions). If all eigenvalues of $J\left(x^{*}\right)$ have real parts less than zero, then the steady point $x^{*}$ is asymptotically stable. If some eigenvalues of $J\left(x^{*}\right)$ have positive real parts while the others have negative real parts, then the steady point $x^{*}$ is unstable. If at least one eigenvalue of $J\left(x^{*}\right)$ is purely imaginary, one needs to consider non-linear terms of the Taylor series of $f(x)(1.1)$, which we will not go into here.

\section{The game dynamics}

Depending on the actual problem, different kinds of evolutionary dynamics may be appropriate. In this thesis we only use the replicator dynamics and the Lotka-Volterra dynamics. Traditionally, the former dynamics describe interactions within one population consisting of different types while the latter dynamics focus on interactions between multiple populations.

Replicator dynamics The replicator dynamics were introduced by Taylor and Jonker (1978). Suppose there is one population consisting of $n$ types, and the type distribution of this population is denoted by $x=\left(x_{1}, \ldots, x_{n}\right)$ and $\sum_{i=1}^{n} x_{i}=1$. Let $f_{i}(x)$ denote the fitness of type $i \in\{1, \ldots, n\}$ and $\bar{f}(x)=\sum_{i=1}^{n} x_{i} f_{i}(x)$ the average fitness for any type. The replicator dynamics describe that the per capita growth rate of type $i$ at any time ${ }^{7}$ is proportional to the difference between $f_{i}(x)$ and $\bar{f}(x)$

$$
\dot{x}_{i}=x_{i}\left(f_{i}(x)-\bar{f}(x)\right) \text {, for all } i=1,2, \ldots, n .
$$

This is the general form of the replicator dynamics. If we assume players are engaging in the population game defined by an $n \times n$ fitness matrix $A$, then $f_{i}(x)=e_{i} A x^{\top}$ (where $e_{i}$ is the $i$-th row of an $n \times n$ identity matrix). We can write the replicator dynamics as

$$
\dot{x}_{i}=x_{i}\left(e_{i} A x^{\top}-x A x^{\top}\right), \text { for } i=1,2, \ldots, n \text {. }
$$

As discussed earlier, when the time derivative in (1.4) is zero, we are at a steady point $x^{*}$, which can either be stable or unstable. But how does this dynamic stability relate to the

\footnotetext{
${ }^{7}$ Where clear from the context, we will suppress the dependence on time $t$ in the equations to follow.
} 
concept of evolutionary stability, initially introduced as a measure which we can calculate directly from the fitness matrix $A$ ? It can be shown that the two concepts do not always overlap (Hofbauer and Sigmund (1998); Cressman (2003); Hofbauer and Sigmund (2003)). If the steady points are dynamically stable, then they are Nash equilibria, e.g., in Game 2 the mixed (population) strategy $\left(\frac{2}{3}, \frac{1}{3}\right)$ is a stable steady point (let's write $x^{*}=\left(\frac{2}{3}, \frac{1}{3}\right)$ ) of the corresponding replicator dynamics (1.4), and $\left(x^{*}, x^{*}\right)$ should be a Nash equilibrium of Game 2 as well. However, the converse does not always hold. There are Nash equilibria which do not correspond to stable steady points. For example, the coordination game described in Table 1.3 has one mixed-strategy Nash equilibrium $\left(\frac{1}{2}, \frac{1}{2}\right)$, which is no stable steady point of the replicator dynamics given by (1.4) (i.e., neither neutrally stable nor asymptotically stable), and the game dynamics (given by (1.4) starting close to the mixed-strategy Nash equilibrium) will converge to one of the two pure-strategy Nash equilibria.

Regarding evolutionary stability, we can state that ESSs are asymptotically stable (or attractors) but the converse is not always true. There are dynamical attractors which are not ESSs. We provide a well-examined game by Zeeman (1980); Bomze (1983); Hofbauer and Sigmund (1998) with the following fitness matrix

$$
\left(\begin{array}{ccc}
0 & 6 & -4 \\
-3 & 0 & 5 \\
-1 & 3 & 0
\end{array}\right) .
$$

The state trajectories of (1.4) with fitness matrix (1.5) are shown in the unit simplex in Figure 1.1. There are two attractors, one is at the center $\left(\frac{1}{3}, \frac{1}{3}, \frac{1}{3}\right)$ and the other one is at the point $(1,0,0)$ (the full red circles). Other steady points include one repeller $(0,1,0)$ (the open circle) and three saddle points $(0,0,1),\left(\frac{4}{5}, 0, \frac{1}{5}\right),\left(0, \frac{5}{8}, \frac{3}{8}\right)$ (the full black circles). We can calculate that $(1,0,0)$ is an ESS by using the first condition for examining evolutionary stability. However, the strategy $\left(\frac{1}{3}, \frac{1}{3}, \frac{1}{3}\right)$ can be invaded by $(1,0,0)$, and this implies that $\left(\frac{1}{3}, \frac{1}{3}, \frac{1}{3}\right)$ is not evolutionary stable. Alternatively, we can rule out $\left(\frac{1}{3}, \frac{1}{3}, \frac{1}{3}\right)$ as an ESS by using the Bishop-Cannings Theorem ${ }^{8}$ (Bishop and Cannings, 1978; Maynard Smith, 1982): If $(1,0,0)$ is an ESS in game 1.5 , then $\left(\frac{1}{3}, \frac{1}{3}, \frac{1}{3}\right)$ cannot be an ESS of this game.

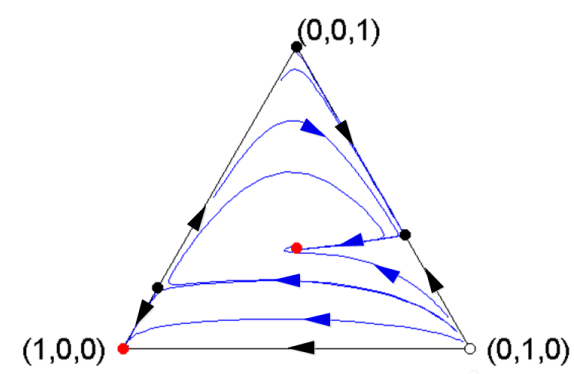

Figure 1.1: Trajectories of the game with an attractor not coinciding with an ESS, depicted in the simplex. The vertices correspond to populations consisting of just one type.

\footnotetext{
${ }^{8}$ The Bishop-Cannings Theorem forbids the occurrence of interior (of the simplex) and boundary ESSs at the same time.
} 
Lotka-Volterra dynamics For our purposes, we will confine ourselves to the LotkaVolterra predator-prey model. This model is commonly used for studying the dynamics between two interacting populations: One is referred to as the predator and another one as the prey. The Lotka-Volterra model that we will introduce here is also sometimes referred to as the predator-prey model, while other predator-prey models exist and not all Lotka-Volterra systems of equations describe predator-prey dynamics.

The Lotka-Volterra model was proposed separately by Lotka (1926) and Volterra (1927).

Formally, let $y_{1} \geq 0$ and $y_{2} \geq 0$ be the prey and predator population sizes, respectively. The per capita growth rates of the two population sizes over time are given as

$$
\begin{aligned}
& \dot{y}_{1}=y_{1}\left(\alpha-\beta y_{2}\right) \\
& \dot{y}_{2}=y_{2}\left(\delta y_{1}-\gamma\right),
\end{aligned}
$$

where $\alpha$ (the growth rate of the prey, in the absence of the predator), $\beta$ (the predation rate), $\delta$ (the rate at which the predator is consuming the prey), and $\gamma$ (the mortality rate of the predators) are positive constants. In the absence of prey, the predator population would decay exponentially to zero due to starvation; but it increases with the prey population. In the absence of predators, the prey population would grow exponentially; but its growth rate decreases linearly as a function of the predator population.

Even though the Lotka-Volterra predator-prey dynamics were introduced earlier than evolutionary game theory was introduced, the dynamical system may fit well to describe evolutionary dynamics generated from predator-prey games. In such games, the predator and the prey populations interact with each other as two players using two strategies, respectively, and through such interactions players influence each other's chances of survival (as fitnesses). For instance, a scarcity of prey will cause the predator population to decrease; as a result, the prey population will increase. This, in turn, will cause the predator population to increase, eventually leading to a decline in the prey, etc.

The model (1.6) can be extended to describe the dynamics of $n$ interacting populations. The $n$ interacting populations can be also referred to as $n$ types. Formally, let $r_{i}$ be the intrinsic growth/decay rate of type $i \in\{1,2, \ldots, n\}$, let each entry of $a_{i j} \in \mathbb{R}$ denote the effect of type $j \in\{1,2, \ldots, n\}$ on type $i$, i.e., type $j$ is enhancing, indifferent to, or inhibiting the growth of type $i$ if $a_{i j}$ is positive, zero, or negative, respectively. We arrive at the generalized predator-prey model

$$
\dot{y}_{i}=y_{i}\left(r_{i}+\sum_{j=1}^{n} a_{i j} y_{j}\right), \text { for } i=1,2, \ldots, n \text {. }
$$

We consider $A=\left(a_{i j}\right)$ being the payoff (or the fitness) matrix, since the effect of type $j$ on type $i$ can be interpreted as a kind of payoff to an individual of type $i$ that results from interacting with an individual of type $j$.

We could calculate the steady points of (1.7), which are useful to analyze the dynamic stability of the predator-prey system for $n$ types. It is possible to convert the replicator dynamics for $n$ types into the Lotka-Volterra model with $n-1$ types, and vice versa. We provide the proof of this equivalence in Appendix A (page 147). Our calculation is based on Hofbauer (1981). 
Other evolutionary dynamics such as adaptive dynamics and best-response dynamics exist and are very interesting to study, however as this thesis focuses mainly on the two dynamics introduced in this section, we will refer the interested reader to Hofbauer and Sigmund (1998) for further study.

\subsection{Spatial games}

In this section we focus on a more recent topic in evolutionary game theory. We introduce the extension of the standard game dynamics (i.e., equations (1.4) and (1.7) in the previous section), which takes into account spatial interactions (of players) in games.

The standard game dynamics hypothesize that the population is well mixed (Bomze, 1983; Malcai et al., 2002; Hauert et al., 2002; Traulsen et al., 2005; Lee et al., 2005; Hofbauer et al., 2009; Hauert, 2010). By a well-mixed population we mean that every individual is equally likely to interact with any other individual in the population. This hypothesis may also imply that an individual either interacts with every other individual or with an average individual which uses the population strategy. In a situation deviating from the well-mixed population hypothesis, interactions depend on space (or a spatial structure) which may not allow one playing against any other individual from the same population. Such space (or spatial structure) dependent interactions are what we call spatial interactions. We will call games that take spatial interactions into account spatial games and standard games nonspatial games.

Spatial games are often used in defining real biological interactions (Boyd and Richerson, 2002; Santos and Pacheco, 2005; Roca et al., 2009; You et al., 2017). In general, there are two approaches to apply spatial models. On the one hand, spatial game dynamics take the form of diffusion processes framed as partial differential equations. The term "diffusion process" may refer to a dispersal process of players, such as migration. On the other hand, interactions can take place over some spatial structures such as grids or graphs. This is usually done via an agent-based approach, where every individual player is treated as a single agent. Dynamics of these agent-based models require specified game rules, which define how each individual player perceives its own surrounding, what information it obtains, and how this information translates into strategy imitation or reproductive success during a play. While it is beyond this thesis to explain all possible spatial games, let us here focus on some of their key representatives.

\section{Games with diffusion processes}

In this approach, game dynamics in space are framed as partial differential equations (Ferriere and Michod, 1995; Wakano et al., 2009; Helbing, 2009). Generally, players are diffusing (i.e., dispersing) randomly in a continuous space and the dynamics are obtained by adding diffusion terms to ordinary differential equations. ${ }^{9}$ For example, the diffusion equations

\footnotetext{
${ }^{9}$ Although in more complex spatial games the players' decisions may be included in the diffusion terms.
} 
regarding the general replicator dynamics (1.3) in a 2-dimensional space are given by

$$
\dot{x}_{i}=d_{i} \nabla^{2} x_{i}+x_{i}\left(f_{i}(x)-\bar{f}(x)\right) \text { for } i=1,2, \ldots, n
$$

where $x_{i}$ denotes the density of type $i$ at a point of a two-dimensional space at time $t, d_{i}$ specifies the diffusion rate for type $i$ and $\nabla^{2} x_{i}$ denotes the diffusion rate of $x_{i}$ in the directions

of the space coordinates $(\chi, \xi)$, i.e., $\nabla^{2} x_{i}=\frac{\partial^{2} x_{i}}{\partial \chi^{2}}+\frac{\partial^{2} x_{i}}{\partial \xi^{2}}$. In this case, the fitness of type $i$ does not merely depend on how type $i$ is playing against an arbitrary type from the population, but also depends on its diffusion rate in the two (or more) directions in space. The space effect incorporated by diffusion equations may change the game dynamics (1.3). Examples include a game of Prisoner's Dilemma with diffusion terms (Wakano and Hauert, 2011) and a Rock-Scissors-Paper game where all three strategies can coexist when diffusion terms are included (Wang et al., 2010). The diffusion equations may be approximated by agentbased simulation of spatial games taking place on certain spatial structures, e.g., Avelino et al. (2014) showed that for a Rock-Scissors-Paper game, dynamics generated by a spatially explicit game over a grid discretizing the continuous two-dimensional space are very close to those of the diffusion equations for the same game.

\section{Games on specific spatial structures}

The well-mixed population hypothesis likely does not apply in many real-life situations. For example, geographical or physical constraints may limit the interactions between players (Axelrod et al., 2002; Onnela et al., 2011; Singh and Marx, 2013). Also, social group formation may challenge the idea of interacting with everyone equally (Ashforth and Mael, 1989; Backstrom et al., 2006; Tajfel, 2010).

Hence, it is adequate to come up with certain discrete-space or continuous-space structures (of the field where games take place) which define who is allowed to interact with whom. Discrete-space games are usually played over a grid composed of identical cells, such as identical squares or hexagons, or vertices in a graph. The individuals are then residing in these cells or vertices and in the simplest case only adjacent individuals are allowed to interact with each other. In a continuous space, individuals can reside at any coordinate of the game field. Two individuals can interact with each other if their coordinates are within a certain distance (Cardillo et al., 2012).

As opposed to nonspatial games, in spatial games the interactions are usually between players and their neighbors. In studies of social behaviors, player payoffs depend on their local interactions and the players may modify their strategies by mimicking their neighbors' strategies in order to get higher payoffs. The corresponding evolutionary outcomes may be very different from those generated by nonspatial game dynamics.

Next, we list some examples of spatial games with local interactions. We first introduce games on grids and (regular) graphs. As a result of structural regularity, spatial games on grids and (regular) graphs enjoy remarkable popularity due to mathematical simplicity and/or simulation efficiency. Examples of evolutionary games on grids include the spatial variants of Prisoner's Dilemma (Nowak and May, 1992; Lindgren and Nordahl, 1994; Hutson 
and Vickers, 1995; Grim, 1996; Nakamaru et al., 1997; Szabó and Tőke, 1998; Brauchli et al., 1999; Cohen et al., 2001; Schweitzer et al., 2002), the Snowdrift game ${ }^{10}$ and the Hawk-Dove game (Killingback and Doebeli, 1996; Hauert and Doebeli, 2004; Sysi-Aho et al., 2005). We are going to introduce a few of these examples. Nowak and May (1992) introduced a spatial version of the standard Prisoner's Dilemma game, in which a player can choose either Cooperation or Defection. They considered the payoff matrix :

$$
\begin{aligned}
& \text { Cooperation Defection } \\
& \begin{array}{l}
\text { Cooperation } \\
\text { Defection }
\end{array}\left(\begin{array}{ll}
1 & 0 \\
q & z
\end{array}\right) \text {, }
\end{aligned}
$$

with $1<q<2$ and $0<z \ll 1$. While the nonspatial dynamics of the Prisoner's Dillema tend to favor the defectors (i.e., full defection of the population is an ESS for the nonspatial game), Nowak and May (1992) demonstrated that local interactions can lead to maintaining cooperation. The authors observed formation of clusters of cooperators on a 2-dimensional grid, which gave cooperators enough payoff to survive when surrounded by some defectors. However, a spatial structure may not always have a positive influence on cooperation. Hauert

\begin{tabular}{|c|c|c|}
\hline & Cooperation & Defection \\
\hline Cooperation & 1 & $2-q$ \\
\hline Defection & $q$ & 0 \\
\hline
\end{tabular}
and Doebeli (2004) studied the following Snowdrift game on a grid:

with $1<q<2$. The authors observed that the promotion of cooperators was only seen for low $q(\approx 1)$, whereas for medium $q(\approx 1.5)$ and high $q(\approx 2)$ cooperation did not prevail. However, based on their results of a spatial Hawk-Dove game on a grid, Killingback and Doebeli (1996) reported that a spatial structure is beneficial to cooperation. The outcomes of the spatial Snowdrift game and the spatial Hawk-Dove game seemed to be contradicting each other. Roca et al. (2009) found out that this was due to different updating rules: Both Nowak and May (1992) and Killingback and Doebeli (1996) applied the imitation rule (i.e., a player always mimics the strategy of a neighbor with the highest payoff), and Hauert and Doebeli (2004) used the pairwise interaction rule (i.e., a player mimics a randomly selected neighbor's strategy with a probability that depends on their payoff difference). Roca et al. (2009) concluded that the updating rule is crucial to spatial games. Similarly, Ohtsuki et al. (2007) suggested that the evolutionary results on graphs may differ from the results obtained by standard game dynamics (e.g., the replicator dynamics) to an extent depending on the updating rule. Ohtsuki et al. (2007) examined classical games (e.g. Prisoner's Dilemma, Snowdrift game or Hawk-Dove game) on a regular graph and examined different updating rules, like the birth-death rule (i.e., the probability of a randomly selected player to reproduce is proportional to its payoff and the offspring replaces its randomly chosen neighbor), the

\footnotetext{
${ }^{10}$ Snowdrift is another name for the Hawk-Dove game. Different names stem from different research areas, while Snowdrift game describes a situation mostly arising in social behavior study: Two car drivers are stuck in a snowdrift. If both refuse to shovel the snow, none of them will get home. If one of them shoves while the other refuses, both will get home. However, the shoveling player pays a cost. If both shovel the snow, both will get home and share the cost (of shoveling).
} 
death-birth rule (i.e., a random player is chosen to die and the chances of its neighbors to occupy the empty space is proportional to their payoffs). Updating rules may also differ in terms of time. Experiments with simultaneous updating (i.e., strategies of all players are updated simultaneously) and sequential updating (i.e., players update their strategies one after another following a certain sequence) may lead to strikingly different evolutionary outcomes. For instance, Nowak and May (1992) claimed that for a Prisoner's Dilemma the cooperation is improved with simultaneous updates in space, whereas Huberman and Glance (1993) showed that for exactly the same game, sequential updates hinder cooperation and drives all players to defect.

These mentioned spatial game-theoretical models have served well to study the evolution of social behaviors. In these models a player's switch from one strategy to another strategy is sometimes considered as death of the player with the original strategy and birth of a player with the new strategy. In this context, the updating rule is interpreted as reproduction. More advanced models, however, introduced empty cells for hosting offspring.

Usually, empty cells are created when individuals die or move (Kerr et al., 2002; Reichenbach et al., 2007; Simpson et al., 2007; Avelino et al., 2014). The grids with or without empty cells, however, may still be limited by further assumptions on the structure of the field and by definition of the neighborhood. One of the common assumptions is that every player has a fixed neighborhood structure.

A continuous space attracts interest of recent research as it allows for a more flexible structure, where players can reside at any point of the game field. For example, players can migrate to any coordinate of the field within a given migration distance and direction (Cardillo et al., 2012), players can reproduce and place their offspring at any random coordinate within the continuous space (Gallaher and Anderson, 2013; Gallaher et al., 2017). In Chapter 3 of this thesis we will discuss differences between the dynamics produced by continuous-space and discrete-space models.

\subsection{An overview of this thesis}

As mentioned earlier, the main topic of this thesis is spatial and nonspatial evolutionary game theory. In Chapters 2, 4, and 5 we show its application in studying specific biological problems, while in Chapter 3 we focus on comparing the dynamics of discrete-space and continuous-space games. Below we list motivation, problem statement as well as our methodology for each chapter.

Chapter 2 introduces a nonspatial game describing summer-season interactions of the predatory mites (Acari: Phytoseiidae, the predator) and the fruit-tree red spider mites (Acari: Tetranychidae, the prey), inspired by studies on the use of the predatory mites for biological pest control of the fruit-tree red spider mites. Apart from interacting, the predatory mites and the fruit-tree red spider mites may also avoid each other by moving into refuges, giving up reproduction, and entering a state of physiological rest, the so-called diapause. The player decisions of "joining" vs. "opting out" the game, may impact their chances of survival. We expand a simple Lotka-Volterra two-dimensional ordinary differential 
equation system for describing the population densities of both species with time-varying decisions, which are maximized with respect to the player fitness functions. We combine analytical and numerical methods for finding these time-varying optimal decisions for the predator and prey to answer the following question:

Research question 1: "Can a straightforward game-theoretical extension of a predatorprey Lotka-Volterra model capture summer-season interactions between the predatory mites (Acari: Phytoseiidae) and the fruit-tree red spider mites (Acari: Tetranychidae) with a sufficient level of realism?"

We discuss whether the optimal decisions that we find correspond to those observed in laboratory and field experiments. We subsequently discuss another model, which was proposed in Staňková et al. (2013a) and includes energy for predator and prey energy dynamics, but it is a more complex approach to address research question 1, because the differential equation system involved is four-dimensional.

When nonspatial games do not capture the critical elements of biological interactions with a sufficient level of realism, then spatial models are needed. In Chapter 3 we focus on a comparison of the dynamics of the two types of spatial games: The discrete-space and the continuous-space game. Usually one of these two spatially explicit models is chosen for studying specific problems, while the reason of choosing such model is usually not discussed. A thorough understanding of the difference between the two types of spatial models is needed in order to decide in which situations these two types of models can be interchanged and in which situations a particular one of the two should be chosen. Moreover, we need to understand how their predictions compare to their nonspatial variants. As a first step to such understanding, in this chapter we ask ourselves the following question:

Research question 2: To what extent do the eco-evolutionary dynamics of the continuousspace model, discrete-space model, and replicator dynamics differ from each other and why?

We introduce discrete-space and continuous-space games as spatial extensions of the wellknown replicator dynamics. We use agent-based simulations to track eco-evolutionary (population and frequency) dynamics. We examine the conditions under which the population and frequency dynamics of the two types of spatial games differ and why. In particular, we observe that the two types of spatial models produce strikingly different population and frequency dynamics when the space is nearly saturated. Moreover, the discrete-space model shows much higher variance in predictions per run when compared to the continuous-space model. The results of this chapter may help researchers who need a suggestion on selecting a proper spatial model for the problem of their interest.

Chapter 4 demonstrates an application of discrete-space games in studying mechanisms that lead to microbial coexistence. Huge numbers of microbes coexist in almost all habitats of our planet. Not only their high numbers, but also their very high species diversity raise an obvious question: How is the diversity maintained? Understanding how microbes interact with each other is important for maintaining the homeostasis within the human body. Within this chapter, we address the following question: 
Research question 3: "When modeling microbial interaction by means of a discrete-space model, what impact do the so-called interaction matrix (determining whether or not a microbial individual of a particular species kills its neighbor by toxin secretion), the neighborhood size and the interaction rules have on the rate of coexistence for different types of players?"

To do this we implement a number of scenarios to experiment with different interaction rules and different neighborhood sizes. For each of these simulation experiments, we examine the types that coexist in the population at the end of the runs. These models are inspired by interaction matrices derived in several laboratory experiments on pairwise competition between different types of microbes. Microbial interactions are usually local, thus a spatial game lends itself better to study such interaction mechanisms. The game is played over a discrete-space field composed of identical cells, as bacteria are assumed to be of a more or less identical morphology. Other motivations for choosing this particular spatial model when describing microbial interactions are discussed in this chapter as well. We use agent-based simulations to mimic several scenarios of microbial interactions and analyze whether or not the interacting strains coexist. We compare predictions of models on a square grid with those on a hexagonal grid to understand the relation between the coexistence rate and the neighborhood size.

In Chapter 5 we model tumorigenesis in a metastatic castrate-resistant prostate cancer (mCRPC) as a continuous-space game. Understanding how tumors grow and how various types of cancer cells interact in space is essential for designing a successful cancer treatment. In our continuous-space game, we analyze the interaction of three different types of cancer cells. Based on state of the art cancer biology for these types, 22 potential fitness matrices are examined. Moreover, we examine the impact of three radii that influence the eco-evolutionary (population and frequency) dynamics. These radii are (1) the frequencydependence radius within which cells interact with each other, (ii) the density-dependence radius within which cells experience limits to population growth, and (iii) the dispersal radius which determines the region in which daughter cells are being put in the field. Therefore, we can formulate the main question to be answered in this chapter as follows:

Research question 4: "What is the impact of the choice of fitness matrix on the observed processes and in how far do these processes depend on the three radii mentioned before?"

In our model, each individual cancer cells represents a player, that can be of three different types (differing in the way they react to testosterone). Together, these players make up a population. Pairwise interactions between cells lead to cell proliferation according to a specific fitness matrix. We use agent-based simulations to track the evolution of the spatial (population and frequency) dynamics. We examine the spatial (population and frequency) dynamics at stable equilibria and compare them to the corresponding nonspatial ESSs. We investigate the reasons behind differences in the predictions of the nonspatial and spatial models and search conditions under which their predictions coincide. 
Finally, Chapter 6 concludes this thesis by summarizing the results derived and by discussing future research directions.

\subsection{Reading guidelines}

Here we conclude Chapter 1: Introduction in which we explained the main concepts of standard (evolutionary) game theory and summarized some recent extensions relevant for this thesis. Figure 1.2 presents an overview of how the chapters of this thesis relate to each other. Chapters 2 to 5 can be read more or less independently of the others. Chapter 2: Joining vs. opting out a predator-prey game addresses the nonspatial predator-prey game between the predatory mites (Acari: Phytoseiidae) and the fruit-tree red spider mites (Acari: Tetranychidae). Then, Chapter 3: Discrete-space vs. continuousspace games discusses the differences between discrete-space and continuous-space games and serves as an introduction to discrete-space and continuous-space evolutionary games in Chapters 4 and 5 respectively. Chapter 4: A game-theoretical approach to microbial coexistence studies modeling assumptions that influence conclusions on microbial coexistence and Chapter 5: Spatial and nonspatial games of tumorigenesis studies a modeling approach to tumorigenesis in prostate cancer. Finally, Chapter 6: Conclusions summarizes the results of this thesis and discusses possible directions for future research.

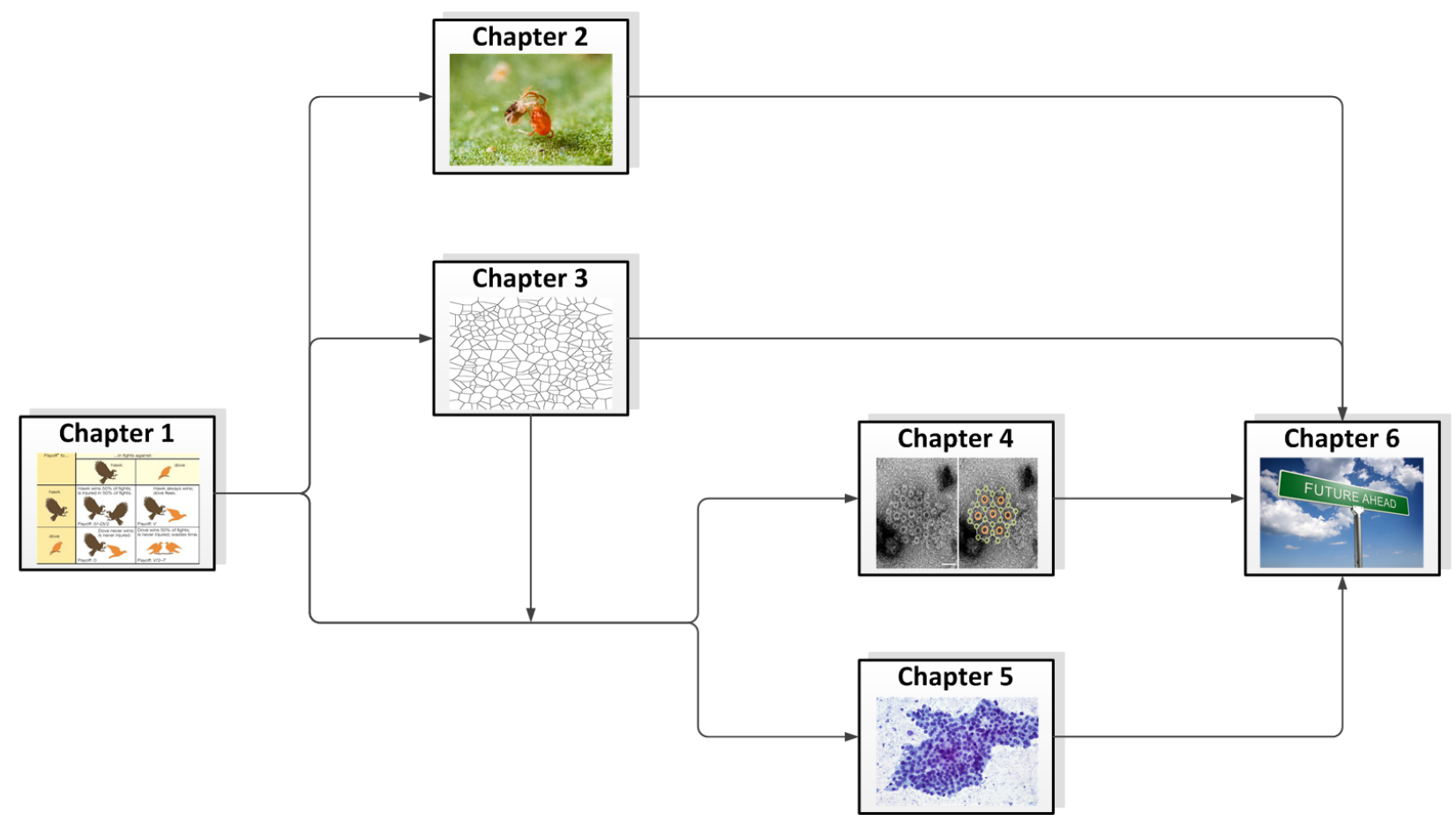

Figure 1.2: Schematic representation of the guidelines for the readers. Arrows represent the connections between chapters. 


\section{Joining vs. opting out a predator-prey}

game

\section{Contents}

2.1 Introduction $\ldots \ldots \ldots \ldots \ldots \ldots \ldots \ldots \ldots \ldots \ldots \ldots \ldots$

2.2 The predator-prey game between the predatory mites and the fruit-tree red spider mites ............... 27

2.2.1 Observations regarding the system of the predatory mites and the fruit-tree red spider mites . . . . . . . . . . . 27

2.2 .2 The model . . . . . . . . . . . . . . . 27

2.2.3 Hamilton-Jacobi-Bellman approach to find optimal strategies for the predator and the prey . . . . . . . . . . . 29

2.3 Another way to model the problem $\ldots \ldots \ldots \ldots$

2.4 Conclusion \& discussion $\ldots \ldots \ldots \ldots \ldots \ldots$ 


\section{Abstract}

Apart from interacting, predator and prey may also avoid each other by moving into refuges where they may lack food, yet survive by switching to an energy-saving physiological state, the so-called diapause. Standard Lotka-Volterra dynamics of predator-prey interactions do not include this option. In this chapter, we model the game of "joining-vs-opting-out" by extending the Lotka-Volterra model to describe summer-season interactions in the real predator-prey system of the predatory mites (Acari: Phytoseiidae) and the fruit-tree red spider mites (Acari: Tetranychidae; the prey mites). In this population game, the predator and the prey populations are the players and the time-varying frequencies of those "joining" and "opting out" the game are their strategies, where both populations maximize their chances of survival within the season. The model includes only two differential equations describing the population densities of the predator and the prey, which depend on their strategies. The goal is to see whether this relatively simple model can depict the interactions of interest with a sufficient level of realism. The optimal strategies are found for general parameter values using the Hamilton-Jacobi-Bellman approach. While this analysis cannot be completed due its complexity, the optimal strategies which we obtain from this analytical approach certainly do not match those observed in laboratory and field studies. This suggests that the proposed model is too simplistic to capture the essentials of the interactions at hand. Subsequently, we include numerical computations for specific parameter values to find the optimal strategies in the specific instances of the game, which support our conclusions that the optimal strategies in this game do not coincide with those observed in the real system. Therefore, we discuss another model, which includes energy dynamics of mean predator and prey individuals and hence transforms the problem into an energy-allocation type of game, which leads to optimal strategies matching empirical observations. Therefore, we conclude that including energy dynamics into the predator-prey game is a parsimonious manner for describing the system of interest with a sufficient level of realism while keeping the model simple.

Keywords: Diapause, Mites, Game-theoretical models, Predator-prey interactions, Nonspatial games

Adapted from: Staňková Kateřina, Abate Alessandro, Sabelis Maurice W., Buša Ján, You Li, 2013. Joining or opting out of a Lotka-Volterra game between predators and prey: Does the best strategy depend on modeling energy lost and gained? Interface focus, 3(6), paper nr. 20130034. 


\subsection{Introduction}

The predator-prey model, proposed independently by Lotka (1926) and Volterra (1927), assumes that predator and prey are continuously exposed to each other and therefore interact all the time. In reality, however, predator and prey also have an option to avoid interacting with each other. They may then move into refuges that are free of their enemies, but may lack food, and where survival may demand a switch to an energy-saving physiological state, such as the so-called diapause (Danks, 1987; Veerman, 1992). Diapause has always been thought to emerge solely to overcome the winter season and to emerge in response to abiotic factors signaling the onset of the winter season, but it may also emerge in response to food scarcity and/or the risk of predation, as it is believed to happen in the system of the so-called predatory mites (Acari: Phytoseiidae) and the fruit-tree red spider mites (Acari: Tetranychidae; prey mites) (Tauber et al., 1986; Danks, 1987; Veerman, 1992, 1994, 1985; Kroon et al., 2004, 2005, 2008). Although empirical proof of these diapause-governing principles is limited, they may well hold more generally for ecological interactions in many other systems.

The work of this chapter was inspired by studies on the use of the predatory mites (Acari: Phytoseiidae) for biological pest control of the fruit-tree red spider mites (Acari: Tetranychidae) that feed on and damage leaves of apple trees (Helle and Sabelis, 1985). This system is seasonal: Each year is divided into two parts, the summer and the winter seasons. The summer season lasting for about five months alternates with the winter season, lasting for about seven months (Helle and Sabelis, 1985). Winters are usually harsh and as such endanger the survival of the prey mites (Helle and Sabelis, 1985) and (even more so) that of the predatory mites (Fitzgerald and Solomon, 1991). The predatory mites and the fruit-tree red spider mites can consume food only during the summer season. While for both the predatory mites and the fruit-tree red spider mites the number of those entering a summer season is a fraction of those entering the previous winter season, in the case of the prey individuals all those which are not in diapause when the summer season ends will die, while those in diapause can survive. Therefore, the decision to enter diapause promotes the survival of fruit-tree red spider mites during winter. As without natural enemies, this diapause emerges in response to abiotic factors signaling the onset of the winter season, such as sufficient night length or low temperatures, it can be relatively easily induced in a greenhouse (Veerman, 1992, 1994). However, using another but similar species of spider mites, the two-spotted spider mite (Acari: Tetranychidae), it was shown that the decision to enter diapause also depends on abundance of its predators during summer (Kroon et al., 2004, 2005, 2008). From the point of view of the prey mite, going into diapause in response to predation risks makes intuitive sense as it faces a grim future with increasing predator densities and thus an increased risk of death: it may then do better by giving up reproduction, moving away from leaves to twigs and branches, and entering diapause. Then, if many prey mites would make the same decision, this could create a negative feedback on the population of the predatory mites, so that at some point in time the prey mites would profit from the decreased predation risk by terminating their diapause and returning to the leaves. However, 
such a behavior in predators is observed only very rarely as predators usually do not enter the diapause in the summer season at all.

An early diapause raises the demands on the energy storage (fat resources) of the fruittree red spider mites, as these resources need to cover a longer period without food before terminating diapause at the beginning of the next summer season - the energy level at diapause termination will determine the reproductive capacity of the prey mite (Kroon et al., 2005). Thus, the decision to enter diapause within a summer season will depend on the current internal energy storage of the prey mite, as this will have far-reaching consequences for winter survival and reproduction in the summer season of the next year. Given the negative feedback between the predator and the prey in this system and the complexity of the decisions that prey mites are faced with, it is virtually impossible to intuitively pinpoint the most likely strategies that will emerge from natural selection. However, it is believed that the diapause of the fruit-tree red spider mites within one summer season is irreversible: Once they enter the diapause, they are very likely to stay there, mainly because the diapause is a very complicated and costly process for the prey mites. All observations suggest that while the predatory mites enter a winter season being active, the prey mites are likely to be all in diapause (Helle and Sabelis, 1985; Tauber et al., 1986; Danks, 1987; Veerman, 1992, 1994, 1985; Kroon et al., 2004, 2005, 2008).

While it is theoretically possible for the predatory mites to flexibly enter and leave diapause during the summer season, the laboratory and field studies suggest that this option is very rare and that the predatory mites are likely to stay active the entire summer season and enter the diapause only when the winter season starts (Danks, 1987).

In this chapter, we will analyze a game-theoretical model that emerges as a plausible extension of the Lotka-Volterra two-species competition model, while keeping the ODE system two-dimensional. This game-theoretical model allows for entering diapause for both predator and prey. The fitness functions for both species reflect the finite horizon of the interactions. Adopting an optimal control approach (Melikyan, 1998), we will find the optimal strategies for the predator and prey, which are the strategies maximizing their fitness function for any choice of the other player, and can be therefore thought of as best responses for the predator and prey in the game. Our goal is to see what behaviors for the predator and the prey are optimal with respect to fitness functions that we predefine; we do not analyze whether these strategies are resistant against invasion by a rare mutant here. Subsequently, we will discuss the applicability of the model for describing the interactions in the system of interest.

The remainder of this chapter is structured as follows. In Section 2.2 we formulate the interactions between the predatory mites and the prey mites as a simple game between the two populations and analyze its optimal strategies. In Section 2.3 we discuss another model which could describe the interaction of interest in a more realistic manner, while being still analytically tractable. Finally, in Section 2.4 we discuss the results of this chapter and propose directions for future research. 


\subsection{The predator-prey game between the predatory mites and the fruit-tree red spider mites}

\subsubsection{Observations regarding the system of the predatory mites and the fruit-tree red spider mites}

During the winter season the predatory mites (Acari: Phytoseiidae) and the prey mites (Acari: Tetranychidae) do not interact with each other and their densities are expected to decrease monotonically. Therefore, in this chapter we will focus on the summer interactions when the predatory mites and the fruit-tree red spider mites interact and consume food, which is essential for their reproduction. Moreover, they can enter diapause to protect themselves from the environment, predation, or lack of food.

We will formulate the interactions between the predatory mites and the fruit-tree red spider mites during one summer season as a differential game over a finite horizon $[0, \Gamma]$ with $\Gamma>0$ being the length of the summer season, with populations of the two different species of mites being the players. The two options, "joining" and "opting out" the game, define mixed strategies for the two populations at each time $t \in[0, \Gamma]$. More precisely, the strategies of the predatory mites and the fruit-tree red spider mites are their portions joining the game at time $t$, denoted by $u^{P}(t)$ and $u^{R}(t)$, respectively. ${ }^{11}$ Both players maximize their fitness expressing their chances of survival until the next summer season, which we will define mathematically in the next section. Seeking their optimal strategies, maximize their fitness functions for any choice of the other player, is a key element of the game analysis.

\subsubsection{The model}

This model is a straightforward extension of the classical Lotka-Volterra model enriched by the strategies. For the notation used in this model, see Table 2.1. The game dynamics are

\begin{tabular}{|l|l|}
\hline parameter & interpretation \\
\hline$\Gamma$ & length of the summer season \\
$R(t)$ & fruit-tree red spider mite (prey) population density at time $t \in[0, \Gamma]$ \\
$P(t)$ & predatory mite population density at time $t \in[0, \Gamma]$ \\
$u^{R}(t)$ & the strategy of the prey population at time $t \in[0, \Gamma]$ \\
$u^{P}(t)$ & the strategy of the predatory population at time $t \in[0, \Gamma]$ \\
$\beta_{R}$ & death rate factor for the prey population, due to predation \\
$\alpha_{P}$ & death rate factor for the predatory population \\
$\alpha_{R}$ & feeding rate factor of the prey mites, on apple-tree leaves \\
$\beta_{P}$ & feeding rate factor of the predatory mites, on the prey mites \\
\hline
\end{tabular}

Table 2.1: Notation of the game-theoretical model

\footnotetext{
${ }^{11}$ Alternatively, we could think of a strategy of a mean individual at time $t$ being aggregated into the strategy of the corresponding population.
} 
described as follows:

$$
\begin{aligned}
& \frac{\mathrm{d} P}{\mathrm{~d} t}=-\alpha_{P} u^{P} P+\beta_{P} u^{P} u^{R} P R, \\
& \frac{\mathrm{d} R}{\mathrm{~d} t}=\alpha_{R} u^{R} R-\beta_{R} u^{P} u^{R} P R .
\end{aligned}
$$

Here $\alpha_{P}>0$ is the death rate factor for the predators, $\alpha_{R}>0$ is the feeding rate factor for the prey, $\beta_{P}>0$ is the population increase rate factor for the predators based on feeding and $\beta_{R}>0$ is the population decrease rate factor for the prey due to predation. The predator and prey decision variables $u^{P} \in[0,1]$ and $u^{R} \in[0,1]$, respectively, are the proportion (frequency) of those being active, as opposed to being in diapause, the state of physiological rest. In (2.1)-(2.2), it is expected that the predator and the prey can die only when they are active, assuming that death rate of diapaused individuals is negligible on the population level. Therefore, if all predatory mites are in diapause, their number does not change. If they are active, they need to feed on active prey mites in order to increase their number. Herbivory can take place only if the fruit-tree red spider mites are active while the prey mites in diapause stay alive. Therefore, if all prey mites are in diapause, their number does not change. If some of the predatory mites are active, the difference between the first and the second term in (2.2) indicates whether their number will decrease or increase. We assume that $0<\alpha_{P} \leq \beta_{P}, 0<\beta_{R} \leq \alpha_{R}$, and $1 \leq P(0), R(0)$.

The predators choose an optimal strategy $u^{P, *}(t) \in[0,1]$ for $t \in[0, \Gamma]$, so that for any choice of $u^{R}(\cdot)$

$$
u^{P, *}=\arg \sup _{u^{P}(\cdot)} P(\Gamma),
$$

while the optimal strategy $u^{R, *}(t) \in[0,1]$ for the prey mites satisfies

$$
u^{R, *}=\arg \sup _{u^{R}(\cdot)} \int_{0}^{\Gamma}\left(1-u^{R}(t)\right) R(t) \mathrm{d} t
$$

for any choice of $u^{P}(\cdot)$. The optimal strategies for the predator and prey coincide with their best response to any strategy of the other player with respect to its fitness function.

The arguments of (2.3) and (2.4) are the fitness functions for the predatory mites and the prey mites, respectively. For the predatory mites, their fitness is defined as the number of individuals that are alive at the end of the summer season (for preparing survival through the upcoming winter season). For the prey mites, their fitness is defined as the number of individuals which enter the diapause at any given time through the summer season, and the longer in diapause they are, the higher chance of survival they have. Note that the fitness function (2.4) is a fitness function for the entire prey population and therefore does not take into account an individual behavior of a single mite which can become active once it has entered diapause.

Thoughts on stability of the system $(2.1)-(2.2)$

We linearize the game dynamics (2.1)-(2.2) for fixed values of $u^{P}$ and $u^{R}$ and analyze the linear stability of the steady points of such a system: The equations (2.1) and (2.2) have 
two pairs of steady points when $u^{P}$ and $u^{R}$ are nonzero: $\left\{P^{*, 1}=R^{*, 1}=0\right\}$ and $\left\{P^{*, 2}=\right.$ $\left.\frac{\alpha_{R}}{\beta_{R} u^{P}}, R^{*, 2}=\frac{\alpha_{P}}{\beta_{P} u^{R}}\right\}$. The Jacobian of the system $(2.1)-(2.2)$ is

$$
J=\left[\begin{array}{cc}
-\alpha_{P} u^{P}+\beta_{P} u^{P} u^{R} R & \beta_{P} u^{P} u^{R} P \\
-\beta_{R} u^{P} u^{R} R & \alpha_{R} u^{R}-\beta_{R} u^{P} u^{R} P
\end{array}\right]
$$

Eigenvalues of $J$ at the steady point $\left\{P^{*, 1}=R^{*, 1}=0\right\}$ are $\alpha_{R} u^{R}$ and $-\alpha_{P} u^{P}$. Eigenvalues of $J$ at the steady point $\left\{P^{*, 2}=\frac{\alpha_{R}}{\beta_{R} u^{P}}, R^{*, 2}=\frac{\alpha_{P}}{\beta_{P} u^{R}}\right\}$ are $i \sqrt{\alpha_{R} u^{R} \alpha_{P} u^{P}}$ and $-i \sqrt{\alpha_{R} u^{R} \alpha_{P} u^{P}}$. If both $u^{P} \equiv 0$ and $u^{R} \equiv 0$, the system dynamics are neutrally stable everywhere, as the right-hand sides of equations (2.1) and (2.2) both stay zero. If $u^{P}$ is nonzero and $u^{R}$ is zero, then the steady points are all points of the form $\left(P^{*}=0, R^{*}\right.$ arbitrary); these are all stable. If $u^{P}$ is zero and $u^{R}$ is nonzero, then the steady points are all points of the form $\left(P^{*}\right.$ arbitrary, $\left.R^{*}=0\right)$; these are all unstable.

\subsubsection{Hamilton-Jacobi-Bellman approach to find optimal strategies for the predator and the prey}

Firstly, we formulate the problem of the predator and the problem of the prey via HamiltonJacobi-Bellman equations (Bellman, 1957). Starting from the end of the season and proceeding backwards in time, we will then study the best reply of the prey to any choice of the predator and the best reply of the prey to any choice of the prey. This way, we will compute optimal behaviors for both of them. While this way we consider a priori that this game is a Nash game, we will see that its results coincide with a Stackelberg game in which the predator as a leader can impose its decision on the prey.

As usual, the analysis is carried out in reverse time, i.e., proceeding from the end of the summer season towards its beginning, we will study the optimal behavior of the predator mites and the prey mites, taking advantage of the principle of optimality (Başar and Olsder, 1995; Melikyan, 1994; Metz et al., 2016).

\section{Characteristic system for the prey}

Let us introduce a reverse time $\tau=\Gamma-t$ (for which it follows that $f^{\prime} \stackrel{\text { def }}{=} \frac{\mathrm{d} f}{\mathrm{~d} \tau}=-\dot{f}$ ) and a value function for the prey

$$
V_{R}(t)=\int_{\Gamma-t}^{\Gamma}\left(1-u^{R}\right) R \mathrm{dt}^{\prime}
$$

With $b_{P} \stackrel{\text { def }}{=} \frac{\partial V_{R}}{\partial P}, b_{R} \stackrel{\text { def }}{=} \frac{\partial V_{R}}{\partial R}$, the Hamilton-Jacobi-Bellman equation (Bellman, 1957) has the form

$$
\begin{aligned}
\mathcal{H}_{R} & =\frac{\partial V_{R}}{\partial t}+\max _{u^{R}}\left(b_{P}\left(-\alpha_{P} u^{P} P+\beta_{P} u^{P} u^{R} P R\right)\right. \\
& \left.+b_{R}\left(\alpha_{R} u^{R} R-\beta_{R} u^{P} u^{R} P R\right)+\left(1-u^{R}\right) R\right) .
\end{aligned}
$$


The characteristic system (in reverse time) is:

$$
\begin{aligned}
& P^{\prime}=\alpha_{P} u^{P} P-\beta_{P} u^{P} u^{R} P R, \\
& R^{\prime}=-\alpha_{R} u^{R} R+\beta_{R} u^{P} u^{R} P R, \\
& b_{P}^{\prime}=b_{P}\left(-\alpha_{P} u^{P}+\beta_{P} u^{P} u^{R} R\right)-b_{R} \beta_{R} u^{P} u^{R} R, \\
& b_{R}^{\prime}=b_{P} \beta_{P} u^{P} u^{R} P+b_{R}\left(\alpha_{R} u^{R}-\beta_{R} u^{P} u^{R} P\right)+1-u^{R},
\end{aligned}
$$

with transversal conditions $b_{P}(0)=0, b_{R}(0)=0$, and assuming $P(0)>0, R(0)>0$.

At the end of the game, the optimal strategy for the prey is to be in diapause, i.e., $u^{R, *}(0)=0$

The optimal decision can then be derived as in Melikyan $(1998,1994)$; Melikyan and Olsder (2010): If $\mathcal{S}_{R}$ is nonzero, then

$$
u^{R, *}=\operatorname{Heav} \mathcal{S}_{R}=\left\{\begin{array}{lll}
1, & \text { if } & \mathcal{S}_{R}>0 \\
0, & \text { if } & \mathcal{S}_{R}<0
\end{array}\right.
$$

with

$$
\mathcal{S}_{R}=R\left(\beta_{P} b_{P} u^{P} P+b_{R}\left(\alpha_{R}-\beta_{R} u^{P} P\right)-1\right) .
$$

Note that the sign of $\mathcal{S}_{R}$ does not depend on $R$, as $R$ is always positive if the prey is alive. As $\mathcal{S}_{R}(0)=-R(0)<0, u^{R, *}$ equals 0 for some interval $\tau \in\left[0, \tau_{1}^{R}\right)$. Note that this may imply that if the season is very short, $u^{R}=0$ is optimal for the prey for the entire season. While the fact that $u^{R, *}(0)=0$ is independent of the behavior of the predator, the (reverse) time when the strategy of the prey should change may depend on the predator strategy $u^{P}$.

This can be seen from the following characteristic system, obtained by substituting $u^{R}=0$ into (2.6)-(2.9) and with initial conditions $b_{P}(0)=b_{R}(0)=0$, where $P(0), R(0) \geq 1$ :

$$
\begin{aligned}
P^{\prime} & =\alpha_{P} u^{P} P, \\
R^{\prime} & =0, \\
b_{P}^{\prime} & =-\alpha_{P} b_{P} u^{P}, \\
b_{R}^{\prime} & =1 .
\end{aligned}
$$

The solution of $(2.11)-(2.14)$ is

$$
b_{P}(\tau)=0, b_{R}(\tau)=\tau, P(\tau)=P(0) \mathrm{e}^{\alpha_{P} \int_{0}^{\tau} u^{P}(\psi) \mathrm{d} \psi}, R(\tau)=R(0)
$$

Substituting this solution into (2.10) leads to:

$$
\mathcal{S}_{R}(\tau)=R(0)\left(\tau \alpha_{R}-\tau \beta_{R} u^{P}(\tau) P(0) \mathrm{e}^{\int \alpha_{P} \alpha_{P} u^{P}(\psi) d \psi}-1\right) .
$$

Note that when this expression for $\mathcal{S}_{R}$ reaches 0 there will be either a discrete switch to $u^{R, *}=1$ or there can be a mixed-strategy $u^{R, *}$. 
Remark 1. (Switching manifold starting at $R(0)=0$ ) Note that in the above analysis we assume $R(0)>0$. This is because if we allowed the condition $R(0)=0$, all prey would be extinct and therefore it would not make sense to look on optimal strategy $u^{R, *}$. However, in theory there could be a switching manifold starting at $R(0)=0$, as $\mathcal{S}_{R}(0)$ would then be equal to zero. In such a situation, prey would either start in diapause or $u^{R} \in(0,1)$. The switching mainfold starting at $R(0)=0$ would then have the following parametrization:

$$
\begin{aligned}
\mathcal{S}_{R}(0) & =0 \\
\mathcal{S}_{R} & =R\left(\beta_{P} b_{P} u^{P} P+b_{R}\left(\alpha_{R}-\beta_{R} u^{P} P\right)-1\right)=0 \\
\mathcal{S}_{R}^{\prime} & =R\left(\beta_{P} b_{P}\left(\frac{\mathrm{d} u^{P}}{\mathrm{~d} \tau}\right) P+\alpha_{R}-\beta_{R} u^{P} P-b_{R} \beta_{R}\left(\frac{\mathrm{d} u^{P}}{\mathrm{~d} \tau}\right) P-b_{R} \beta_{R}\left(u^{P}\right)^{2} \alpha_{P} P\right)=0 .
\end{aligned}
$$

Trivially, these two equations are zero for $R=0$ (i.e., there exists a switching manifold for $R=0)$. However, they are also zero for

$$
\begin{aligned}
b_{P} & =\frac{-\alpha_{R}^{2}+2 \alpha_{R} \beta_{R} u^{P} P-\beta_{R}^{2}\left(u^{P}\right)^{2} P^{2}+\beta_{R}\left(\frac{\mathrm{d} u^{P}}{\mathrm{~d} \tau}\right) P+\beta_{R}\left(u^{P}\right)^{2} \alpha_{P} P}{\beta_{P} P\left(\left(\frac{\mathrm{d} u^{P}}{\mathrm{~d} \tau}\right) \alpha_{R}+\beta_{R}\left(u^{P}\right)^{3} \alpha_{P} P\right)}, \\
b_{R} & =-\frac{-\frac{\mathrm{d} u^{P}}{\mathrm{~d} \tau}-u^{P} \alpha_{R}+\beta_{R}\left(u^{P}\right)^{2} P}{\left(\frac{\mathrm{d} u^{P}}{\mathrm{~d} \tau}\right) \alpha_{R}+\beta_{R}\left(u^{P}\right)^{3} P \alpha_{P}} .
\end{aligned}
$$

Note that this solution makes sense only if $u^{P} \neq 0$. We know that $b_{P}(0)=b_{R}(0)=0$ and $P$ follows the dynamics in (2.6). From the second time derivative of $\mathcal{S}_{R}$ we can obtain an expression for $u^{R}$ along this switching manifold. However, we need to know the expression for $u^{P}$ (in case that $u^{P} \neq 0$ ) in order to be able to get an explicit expression for this intermediate value of $u^{R}$ (as its expression contains $u^{P}$ and its time derivatives). There is a switching manifold present in the dynamics, but only if $u^{P} \in(0,1]$. The expression for $u^{R, *}$ alongside this manifold is then

$$
u^{R, *}=\frac{\psi_{1}}{\psi_{2}}
$$

with

$$
\begin{aligned}
\psi_{1} & =2 \beta_{R} \frac{\mathrm{d} u^{P}}{\mathrm{~d} \tau} P \alpha_{R}-\beta_{R} 2 \frac{\mathrm{d} u^{P}}{\mathrm{~d} \tau} P^{2}\left(u^{P}\right)^{3} \alpha_{P}+\frac{\mathrm{d}^{2} u^{P}}{\mathrm{~d} \tau^{2}} \alpha_{R}{ }^{2}-\frac{\mathrm{d}^{2} u^{P}}{\mathrm{~d} \tau^{2}} \alpha_{R} \beta_{R} u^{P} P \\
& -\frac{\mathrm{d}^{2} u^{P}}{\mathrm{~d} \tau^{2}} \beta_{R}\left(u^{P}\right)^{2} \alpha_{P} P+5 \beta_{R}\left(u^{P}\right)^{2} \alpha_{P} P \frac{\mathrm{d} u^{P}}{\mathrm{~d} \tau} \alpha_{R}+\beta_{R}^{2}\left(u^{P}\right)^{5} \alpha_{P}^{2} P^{2}+\beta_{R}\left(u^{P}\right)^{3} \alpha_{P}{ }^{2} \frac{\mathrm{d} u^{P}}{\mathrm{~d} \tau} \\
& +\beta_{R}\left(u^{P}\right)^{4} \alpha_{P}{ }^{2} P \alpha_{R}+3 \beta_{R} \frac{\mathrm{d} u^{P^{2}}}{\mathrm{~d} \tau} \alpha_{P} u^{P} P, \\
\psi_{2} & =\beta_{R}\left(u^{P}\right)^{2} \beta_{P} P R\left(\frac{\mathrm{d} u^{P}}{\mathrm{~d} \tau} \alpha_{R}+u^{P} \alpha_{P} \frac{\mathrm{d} u^{P}}{\mathrm{~d} \tau}+\left(u^{P}\right)^{2} \alpha_{P} \alpha_{R}\right) .
\end{aligned}
$$

Note that $\psi_{2}=0$ if $u^{P}=0$ which means that as long as $u^{P, *}$ is zero, $u^{R, *}$ is zero as well. Therefore, we can proceed to the analysis of the characteristic system for the predator and only if $u^{P} \in(0,1]$.

Let us now investigate the optimal behavior of the predator at the end of the season, taking into account that $u^{R, *}=0$ at the end of the season as derived earlier. 


\section{Characteristic system for the predator}

Adopting a similar analysis as the one for the prey, we can proceed as follows: We again consider reverse time $\tau=\Gamma-t$ (with $f^{\prime} \stackrel{\text { def }}{=} \frac{\mathrm{d} f}{\mathrm{~d} \tau}=-\dot{f}$ ) and a value function for the predator

$$
V_{P}(t)=\int_{\Gamma-t}^{\Gamma}\left(-\alpha_{P} u^{P} P+\beta_{P} u^{P} u^{R} P R\right) \mathrm{d} t^{\prime}
$$

With $a_{P} \stackrel{\text { def }}{=} \frac{\partial V_{P}}{\partial P}, a_{R} \stackrel{\text { def }}{=} \frac{\partial V_{R}}{\partial R}$, the HJB equation has the following form:

$$
\begin{aligned}
\mathcal{H}_{P} & =\frac{\partial V_{P}}{\partial t}+\max _{u^{P}}\left(a_{P}\left(-\alpha_{P} u^{P} P+\beta_{P} u^{P} u^{R} P R\right)\right. \\
& \left.+a_{R}\left(\alpha_{R} u^{R} R-\beta_{R} u^{P} u^{R} P R\right)-\alpha_{P} u^{P} P+\beta_{P} u^{P} u^{R} P R\right) .
\end{aligned}
$$

The characteristic system is:

$$
\begin{aligned}
& P^{\prime}=\alpha_{P} u^{P} P-\beta_{P} u^{P} u^{R} P R \\
& R^{\prime}=-\alpha_{R} u^{R} R+\beta_{R} u^{P} u^{R} P R \\
& a_{P}^{\prime}=a_{P}\left(-\alpha_{P} u^{P}+\beta_{P} u^{P} u^{R} R\right)-a_{R} \beta_{R} u^{P} u^{R} R-\alpha_{P} u^{P}+\beta_{P} u^{P} u^{R} R \\
& a_{R}^{\prime}=a_{P} \beta_{P} u^{P} u^{R} P+a_{R}\left(\alpha_{R} u^{R}-\beta_{R} u^{P} u^{R} P\right)+\beta_{P} u^{P} u^{R} P,
\end{aligned}
$$

with initial conditions $P(0), R(0) \geq 1$ and transversal conditions $a_{P}(0)=a_{R}(0)=0$. The optimal predator decision can be expressed as

$$
u^{P}=\operatorname{Heav} \mathcal{S}_{P}
$$

with

$$
\mathcal{S}_{P}=a_{P}\left(-\alpha_{P} P+\beta_{P} u^{R} P R\right)-a_{R} \beta_{R} u^{R} P R-\alpha_{P} P+\beta_{P} u^{R} P R
$$

Note that the sign of $\mathcal{S}_{P}$ is independent of $P$, as $P$ has to be positive for the predator to be alive. From the initial and transversal conditions it follows that $\mathcal{S}_{P}(0)=P(0)\left(-\alpha_{P}+\right.$ $\left.\beta_{P} u^{R}(0) R(0)\right)$. As $u^{R}(0)=0, \mathcal{S}_{P}(0)=-\alpha_{P} P(0)<0$. Therefore, $u^{P}(0)=0$. Moreover, we see that while the best response of the prey is a function of the strategy of the predator, the converse is not true. As we can see this phenomenon consistently throughout the analysis, we can conclude that this game has a Stackelberg structure.

Remark 2. (Switching manifold starting at $P(0)=0$ ) Note that in our system it is impossible for $P$ to reach value 0 . If we allowed the condition $P(0)=0$, then there would be a switching manifold starting at $P(0)=0$. Then depending on the initial value of $R$ and value of $u^{R}(0)$, the predator would either start in diapause or $u^{P} \in(0,1)$, which is a contradiction with $P(0)=0$, as then the predator is extinct. Theoretically, if we allow $P(0)=0, R(0)=0$, and $u^{R}(0) \in(0,1)$, then there might be a switching manifold for the predator starting at $P(0)=0$, alongside which $u^{P} \in(0,1)$, while the prey would act as described in Remark 1. In such a case, if $u^{P} \in(0,1)$ at the beginning of the season, the switching manifold for the predator 
would have to satisfy the following conditions:

$$
\begin{aligned}
& \mathcal{S}_{P}(0)=0, \\
& \mathcal{S}_{P}=A p \mathcal{S}_{P}=a_{P}\left(-\alpha_{P} P+\beta_{P} u^{R} P R\right)-a_{R} \beta_{R} u^{R} P R-\alpha_{P} P+\beta_{P} u^{R} P R=0, \\
& \mathcal{S}_{P}^{\prime}=-P R\left(\beta_{P}\left(u^{R}\right)^{2} \alpha_{R}-\beta_{P} \frac{\mathrm{d} u^{R}}{\mathrm{~d} \tau}+a_{R} \beta_{R} \frac{\mathrm{d} u^{R}}{\mathrm{~d} \tau}-a_{P} \beta_{P} \frac{d}{d \tau} u^{R}+a_{P} \beta_{P}\left(u^{R}\right)^{2} \alpha_{R}\right)=0 .
\end{aligned}
$$

From equations $\mathcal{S}_{P}=0, \mathcal{S}_{P}^{\prime}=0$, one can compute that $a_{P}=-1$ and $a_{R}=0$. As with $a_{P}(0)=0$ and dynamics (2.20) $a_{P}=-1$ cannot be achieved, we can conclude that the intermediate strategy and switching manifold starting at $\tau=0$ do not exist. This, however, implies, that also the switching manifold discussed in Remark 1 does not exist.

\section{In reverse time, as long as $u^{R, *}=0$, also $u^{P, *}=0$}

Assuming that $u^{P}(\tau)=u^{R}(\tau)=0$ on some interval $\left[0, \tau_{x}\right)$, and given that $\tau_{x}=\min \left(\tau_{1}^{P}, \tau_{1}^{R}\right)$, where $\tau_{1}^{P}$ and $\tau_{1}^{R}$ are the times at which the predator and prey change the strategy from $u^{P}=0$ to $u^{P} \in(0,1]$ and $u^{R}=0$ to $u^{R} \in(0,1]$ at the end of the season, respectively, we find on this interval that

$$
\begin{aligned}
& P^{\prime}=0, \\
& R^{\prime}=0, \\
& a_{P}^{\prime}=0, \\
& a_{R}^{\prime}=0 .
\end{aligned}
$$

Therefore, for $\tau \in\left[0, \tau_{x}\right), P(\tau)=P(0), R(\tau)=R(0), a_{P}=0, a_{R}=0$, and (from $(2.22)$ )

$$
\mathcal{S}_{P}=-\alpha_{P} P(0)
$$

This means that as long as $u^{R}=0, u^{P}=0$ as well. Moreover, $\mathcal{S}_{P}<0$ also if $u^{P}=0$ and $u^{R}=1$. Therefore, $\tau_{1}^{P}>\tau_{1}^{R}$, while we cannot exclude the option that $\tau_{1}^{P}$ and $\tau_{1}^{R}$ are arbitrarily close to each other.

As long as $u^{P}=0$, the solution to the system (2.11)-(2.14) equals $b_{P}(\tau)=0, b_{R}(\tau)=1$, $P(\tau)=P(0), R(\tau)=R(0)$. Consequently, $\mathcal{S}_{R}=\tau \alpha_{R} R(0)-R(0)$.

This implies that $\mathcal{S}_{R}=0$ if $R(0)\left(\tau \alpha_{R}-1\right)=0$, i.e., the time when $u^{R}=0$ changes into another strategy is equal to $\tau_{1}^{R}=\frac{1}{\alpha_{R}}$ (and is therefore independent of $R$ and $P$ ). Clearly, $P\left(\tau_{1}^{R}\right)=0, b_{R}\left(\tau_{1}^{R}\right)=1, P\left(\tau_{1}^{R}\right)=P(0)$, and $R\left(\tau_{1}^{R}\right)=R(0)$.

If $u^{P}=0$, while $u^{R} \in(0,1)$, the characteristic system for the predator becomes

$$
\begin{aligned}
P^{\prime} & =0, \\
R^{\prime} & =-\alpha_{R} u^{R} R, \\
a_{P}^{\prime} & =0, \\
a_{R}^{\prime} & =a_{R} \alpha_{R} u^{R} .
\end{aligned}
$$

The switching manifold of the predator will become $\mathcal{S}_{P}=P(0)\left(-a_{R}^{s} \beta_{R} u^{R, s} R^{s}-\alpha_{P}+\right.$ $\left.\beta_{P} u^{R, s} R^{s}\right)$, where $R^{s}$ solves (2.28) and $a_{R}^{s}$ solves (2.30), given that $u^{R}=u^{R, s} \in(0,1)$. 
In reverse time, the strategy $u^{R, *}=0$ switches to strategy $u^{R, *}=1$ while $u^{P, *}$ stays at 0

We can use the following relation, with $\{\cdot, \cdot\}$ denoting Jacobi brackets (Melikyan, 1998): $\mathcal{S}_{R}^{\prime}=\left\{\mathcal{S}_{R}, h_{R}\right\}, \mathcal{S}_{R}^{\prime \prime}=\left\{\mathcal{S}_{R}^{\prime}, h_{R}\right\}$, with $h_{R}$ being the Hamiltonian of (2.5). Based on our previous analysis, we assume that $u^{P}(\tau)=0$ until some $\tau_{1}^{P}>0$. Then

$$
\begin{aligned}
& \mathcal{S}_{R}=R\left(\alpha_{R} b_{R}-1\right), \\
& \mathcal{S}_{R}^{\prime}=\left\{\mathcal{S}_{R}, h\right\}=\alpha_{R} R .
\end{aligned}
$$

In other words, as $R \neq 0, \mathcal{S}_{R}^{1}: 0=-b_{R}+\frac{1}{\alpha_{R}}$ is a switching manifold for the prey. Moreover, if there is an intermediate strategy $u^{R, *}=u^{R, s} \in(0,1), \mathcal{S}_{R}=0$ implies $\mathcal{S}_{R}^{\prime}=0$ (Melikyan, 1998). Setting (2.31) and (2.32) to 0 leads to only the trivial solution $R=0$. Therefore, there is no singular strategy for the prey under the assumption that once $u^{R} \neq 0, u^{P}$ is still equal to zero.

Note that if $u^{R, *}$ switched from 0 to a value from $(0,1)$ at $\tau_{1}^{R}$, also co-states $b_{P}$ and $b_{R}$ would go through a discrete switch, thanks to the form of the corresponding HamiltonJacobi-Bellman equation. However, from the expression for $\mathcal{S}_{R}^{1}$ it can be seen that such a switch does not take place.

\section{In reverse time, after $u^{R, *}$ switches to $1, u^{P, *}$ switches to 1 as well}

Let us now investigate the optimal behavior for the predator once $u^{R, *}=1$ if the predator plays $u^{P}=0$. The characteristic system for the predator (2.18)-(2.21) becomes

$$
\begin{aligned}
P^{\prime} & =0, \\
R^{\prime} & =-\alpha_{R} R, \\
a_{P}^{\prime} & =0, \\
a_{R}^{\prime} & =\alpha_{R} a_{R},
\end{aligned}
$$

with initial conditions $P\left(\tau_{1}^{R}\right)=P(0), R\left(\tau_{1}^{R}\right)=R(0), a_{P}\left(\tau_{1}^{R}\right)=0, a_{R}\left(\tau_{1}^{R}\right)=0$. This implies that $a_{P}(\tau)=0, a_{R}(\tau)=0$ also for $\tau>\tau_{1}^{R}, P(\tau)=P(0), R(\tau)=R(0) \mathrm{e}^{-\alpha_{R}\left(\tau-\tau_{1}^{R}\right)}$. Then

$$
\mathcal{S}_{P}=-\alpha_{P} P(0)+\beta_{P} P(0) R(0) \mathrm{e}^{-\alpha_{R}\left(\tau-\tau_{1}^{R}\right)},
$$

which is equal to 0 at time $\tau_{1}^{P}=\left(\tau_{1}^{R}-\frac{\ln \left(\frac{\alpha_{P}}{\beta_{P} R(0)}\right)}{\alpha_{R}}\right)$. Note that if $R(0)=1$ and $\alpha_{P}=\beta_{P}$, then $\tau_{1}^{P}=\tau_{1}^{R}$, otherwise, depending on values of $R(0), \alpha_{P}$, and $\beta_{P}$, the difference between $\tau_{1}^{P}$ and $\tau_{1}^{R}$ might be arbitrarily small or very high.

In order to find the strategy for the predator $u^{P, s} \in(0,1)$, we have to solve the system of characteristic equations (2.18)-(2.21) with $u^{R}=1, u^{P}=u^{P, s}$, leading to:

$$
\begin{aligned}
\mathcal{S}_{P} & =\left(a_{P}+1\right)\left(-\alpha_{P} P+\beta_{P} P R\right)-a_{R} \beta_{R} P R \\
& =P\left(\left(a_{P}+1\right)\left(-\alpha_{P}+\beta_{P} R\right)-a_{R} \beta_{R} R\right)=0, \\
\mathcal{S}_{P}^{\prime} & =\left\{\mathcal{S}_{P}, h\right\}=\beta_{P} P R\left(-a_{P} \alpha_{R}-\alpha_{R}\right)=0 .
\end{aligned}
$$


These two expressions can be equal to zero only for $a_{P}=-1, a_{R}=0$ (note that this outcome coincides with the outcome found in Remark 2). As $a_{P}\left(\tau_{1}^{P}\right)=0$, this is clearly impossible. The conclusion is that at time $\tau_{1}^{P}$ the predator switches to strategy $u^{P}=1$ immediately.

We still need to check whether $u^{R, *}$ changes to another value when $u^{R, *}=1, u^{P, *}=0$. If $u^{R}=1$ and $u^{P}=0$, i.e., for time $\tau \in\left[\tau_{1}^{R}, \tau_{1}^{P}\right)$, the system of characteristics for the prey becomes

$$
\begin{aligned}
& P^{\prime}=0, \\
& R^{\prime}=-\alpha_{R} R, \\
& b_{P}^{\prime}=0 \\
& b_{R}^{\prime}=\alpha_{R} b_{R},
\end{aligned}
$$

with initial conditions $P\left(\tau_{1}^{R}\right)=P(0), R\left(\tau_{1}^{R}\right)=R(0), b_{P}\left(\tau_{1}^{R}\right)=0, b_{R}\left(\tau_{1}^{R}\right)=\tau_{1}^{R}$. Notice that with $u^{P}=0$ the switching manifold can be expressed as

$$
\mathcal{S}_{R}=R\left(\alpha_{R} b_{R}-1\right)
$$

As $b_{R}$ is increasing on $\left[\tau_{1}^{R}, \tau_{1}^{P}\right)$ and $R$ is positive, the prey does not go into diapause from $u^{R}=1$ if $u^{P}=0$. Solving (2.39)-(2.42) yields $P(\tau)=P(0), b_{P}=0, r=R(0) \mathrm{e}^{-\left(\tau-\tau_{1}^{R}\right) \alpha_{R}}$, $b_{R}(\tau)=\tau_{1}^{R} \mathrm{e}^{\left(\tau-\tau_{1}^{R}\right) \alpha_{R}}$.

\section{If $u^{R}=1, u^{P}=1$, will they switch to another value?}

Last but not least, the situation to be examined is when the prey is active and the predator is active as well, i.e., when $\tau>\tau_{1}^{P}$. Behavior of the prey can again be investigated from substituting $u^{R}=1, u^{P}=1$ into the characteristic system (2.6)-(2.9), (2.20)-(2.21):

$$
\begin{aligned}
& P^{\prime}=\left(\alpha_{P}-\beta_{P} R\right) P \\
& R^{\prime}=\left(-\alpha_{R}+\beta_{R} P\right) R \\
& a_{P}^{\prime}=a_{P}\left(-\alpha_{P}+\beta_{P} R\right)-a_{R} \beta_{R} R-\alpha_{P}+\beta_{P} R, \\
& a_{R}^{\prime}=a_{P} \beta_{P} P+a_{R}\left(\alpha_{R}-\beta_{R} P\right)+\beta_{P} P \\
& b_{P}^{\prime}=b_{P}\left(-\alpha_{P}+\beta_{P} R\right)-b_{R} \beta_{R} R \\
& b_{R}^{\prime}=b_{P} \beta_{P} P+b_{R}\left(\alpha_{R}-\beta_{R} P\right)
\end{aligned}
$$

with $P\left(\tau_{1}^{P}\right)=P(0), R\left(\tau_{1}^{P}\right)=R(0) \mathrm{e}^{-\left(\tau_{1}^{P}-\tau_{1}^{R}\right) \alpha_{R}}, b_{P}\left(\tau_{1}^{P}\right)=0, b_{R}\left(\tau_{1}^{P}\right)=\tau_{1}^{R} \mathrm{e}^{\left(\tau_{1}^{P}-\tau_{1}^{R}\right) \alpha_{R}}, a_{P}\left(\tau_{1}^{P}\right)=$ $0, b_{P}\left(\tau_{1}^{P}\right)=0$.

Note also that with $u^{P}=1, u^{R}=1$ the switching manifolds $\mathcal{S}_{P}, \mathcal{S}_{R}$ become:

$$
\begin{aligned}
& \mathcal{S}_{P}=P\left(a_{P}\left(-\alpha_{P}+\beta_{P} R\right)-a_{R} \beta_{R} R-\alpha_{P}+\beta_{P} R\right), \\
& \mathcal{S}_{R}=R\left(b_{P} \beta_{P} P+b_{R}\left(\alpha_{R}-\beta_{R} P\right)-1\right),
\end{aligned}
$$

and they are both positive. Solving (2.44)-(2.48) analytically is impossible, however for different parameter values we could get different behaviors of the optimal strategies in reverse time: 


\begin{tabular}{|l|l|}
\hline parameter & value \\
\hline$\Gamma$ & 10 or 20 \\
$\alpha_{P}$ & 0.05 \\
$\beta_{P}$ & 0.2 \\
$\alpha_{R}$ & 0.25 \\
$\beta_{R}$ & 0.24 \\
\hline
\end{tabular}

Table 2.2: Values of parameters for numerical computations; based on Sabelis (1991); Sabelis and Janssen (1994)

a. As the prey is active, the predator stays active as well, if the decrease of $\mathcal{S}_{R}$ is faster than the decrease of $\mathcal{S}_{P}$. Then, in reverse time, the prey would enter diapause earlier than the predator and the entire analysis could be repeated from the situation $u^{P}=0$, $u^{R}=0$.

b. If at any moment the decrease of $\mathcal{S}_{R}$ becomes slower than the decrease of $\mathcal{S}_{P}$, the predator would enter diapause later than the prey.

When both the predator and the prey are in diapause, we can repeat the analysis carried out so far. Here we have just discussed a partial solution of the problem, focusing on the few first switches of the optimal strategies of the predator and the prey. However, from this analysis it is clear that our model suggests that the predatory mites enter the winter in diapause, which is a clear contradiction to experimental observations of the system. Moreover, it seems that for different parameters a highly varying set of optimal strategies can be obtained.

The typical behavior of the optimal strategies for the predator and the prey follow the pattern in Figure 2.1: The predator may opt out the game before the prey, and the predator and the prey clearly react to each other's strategies.

To show how versatile the optimal strategies can be, in the next section we will focus on numerical calculations with parameters fixed to the values estimated from the laboratory observations. In our analytical analysis we saw that while the best response of the prey is a function of the strategy of the predator, the converse is not true. This shows us that the game has a Stackelberg structure and that is why we assume this structure in the remainder of the chapter.

\section{Numerical case studies}

Next, we focus on a numerical solution to the optimization problem defined in (2.3) and (2.4), subject to (2.1)-(2.2), for a specific set of values, defined in Table 2.2. These values are calculated based on data from Sabelis (1991); Sabelis and Janssen (1994).

The model of the intra-seasonal interaction between the predatory mites and the fruit-tree red spider mites is implemented in Fortran. ${ }^{12}$

\footnotetext{
${ }^{12}$ Fortran was very efficient in the numerical computations for our specific problem (i.e., on the order of seconds), while for example Matlab computed the optimal strategies less efficiently in the order of minutes.
} 


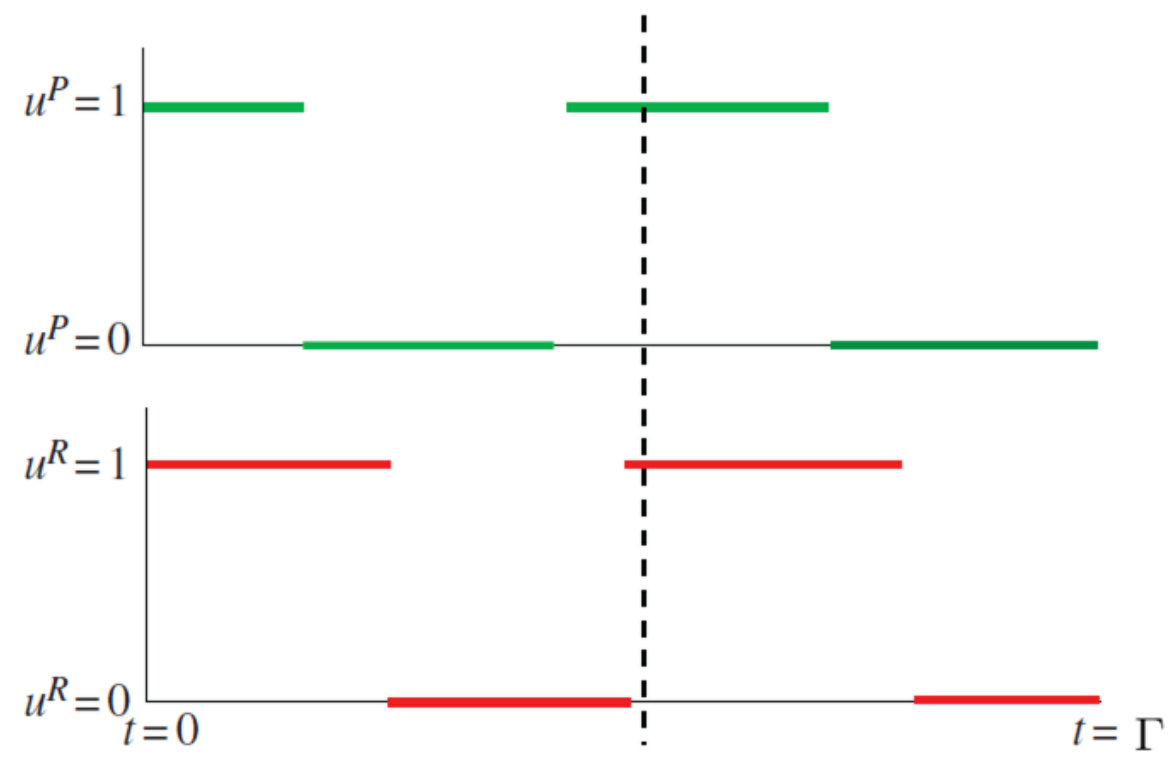

Figure 2.1: The analytical predictions of the optimal strategies in the game-theoretical model, assuming that they are bang-bang, obtained by solving the Hamilton-JacobiBellman's equations for the predator and prey in reverse time. Strategies at the end of the season (to the right of the dashed line): Either the switches between being active and in diapause happen exactly at the same time for both the predator and the prey, but the predator may also switch before the prey does. This would suggest that the predator reacts to the behavior of the prey in reverse time, as opposed to the reaction in the real time, which we would expect. While this observation may be counterintuitive at first glance, it follows from the principle of optimality (Bellman, 1957; Melikyan, 1998). Strategies before the end of the season (to the left of the dashed line): The strategies may be very versatile, which we will investigate in Section 2.2.3.

The time interval $[0, \Gamma]$ is discretized into $\nu$ subintervals. Moreover, in order to search the optimal strategies $u^{P}$ and $u^{R}$, the time interval $[0, \Gamma]$ is discretized into $\varsigma$ subintervals (higher $\varsigma$ increases the precision of the outcome, while $\nu$ has to be divisible by $\varsigma$ ). We let the initial estimates of the optimal strategies $u^{P}$ and $u^{R}$ be random, e.g., initially the optimal $u^{P}$ is a vector consisting of $\varsigma$ random values from $[0,1]$. The ordinary differential system $(2.1)-(2.2)$ is discretized on $\nu$ subintervals using the 4th order Runge-Kutta method (with constant step $\Gamma / \varsigma)$ and subsequently the fitness functions for the predator and the prey are approximated using the trapezoidal rule with the time step $\Gamma / \varsigma$. As we have shown that the game has a Stackelberg structure with the predatory mites as a leader, the constraint optimization for the prey mites was embedded into the constraint optimization of the predatory mites. We have solved the problem via constrained optimization, combining a trust-region algorithm with an active-set based method. Two cases differing in assumptions on the strategies $u^{P}$ and $u^{R}$ were considered:

(a) The optimal $u^{P}$ and $u^{R}$ are assumed to be continuous, piecewise affine functions.

(b) The optimal $u^{P}$ and $u^{R}$ are allowed to have discontinuities in the internal points. 
We will call the optimal $u^{P}$ and $u^{R}$ in (a) and (b) the continuous strategies and discontinuous strategies, respectively. Note that the optimal strategies in the discretized problem may differ from the optimal strategies of the original problem, however we hope that the discretization is fine enough to get close enough to the solutions of the original problem.

Results of the numerical case studies are shown in Table 2.3, where different parameters for the approximation of the optimal results, obtained by discretized numerical techniques, are listed. Table 2.3 includes the cases when the optimal $u^{P}$ and $u^{R}$ are continuous and discontinuous, respectively, where $\Gamma=10$ and $\Gamma=20$, respectively. With the same values of $\nu$ and $\varsigma$ (in continuous and discontinuous cases), higher $P(\Gamma)$ indicates a better outcome for the predator. In Table 2.3 we can see that the discontinuous strategies lead to higher $P(\Gamma)$ than the continuous ones.

The graphs comparing the different outcomes are shown in Figures 2.2-2.3.

The numerically calculated optimal strategies show multiple switches in both predator and prey strategies. In none of the numerical studies that we performed the predator ended the summer season active, often the predator goes into diapause earlier than the prey, which is consistent with the analytical calculations (Figure 2.1).

When the numerically calculated optimal strategies are discontinuous (Figure 2.3), they vary much more frequently between "joining" and "opting out" than in the case when the optimal strategies are continuous (Figure 2.2). Neither the analytically calculated optimal strategies nor the numerically calculated optimal strategies match empirical observations on the diapause behavior of the predatory mites and the fruit-tree red spider mites.

\begin{tabular}{|l|l|l|l|l|}
\hline Strategy type & $\Gamma$ & $\nu$ & $\varsigma$ & $P(\Gamma)$ \\
\hline Continuous & 10 & 100 & 50 & 1.2826 \\
Continuous & 10 & 100 & 100 & 1.2852 \\
Discontinuous & 10 & 100 & 50 & 1.3216 \\
Discontinuous & 10 & 100 & 100 & 1.6193 \\
\hline Continuous & 20 & 200 & 100 & 3.9973 \\
Continuous & 20 & 200 & 200 & 4.2507 \\
Discontinuous & 20 & 200 & 100 & 4.2500 \\
Discontinuous & 20 & 200 & 200 & 4.3878 \\
\hline
\end{tabular}

Table 2.3: Different parameters for the approximation of optimization

Based on the results reported in this section, we conclude that the simple game-theoretical model for predator-prey interactions in the system of the predatory mites (Acari: Phytoseiidae) and the fruit-tree red spider mites (Acari: Tetranychidae), defined by (2.1)-(2.2), does not describe the interactions within this predator-prey system with a sufficient level of realism. Adopting both analytical and numerical methods and comparing the strategies they predict qualitatively with experimental data, we conclude that a more complex model of the interactions will be needed to describe these interactions with a sufficient level of realism. In the next section, we will study a model that was proposed for such a purpose. 

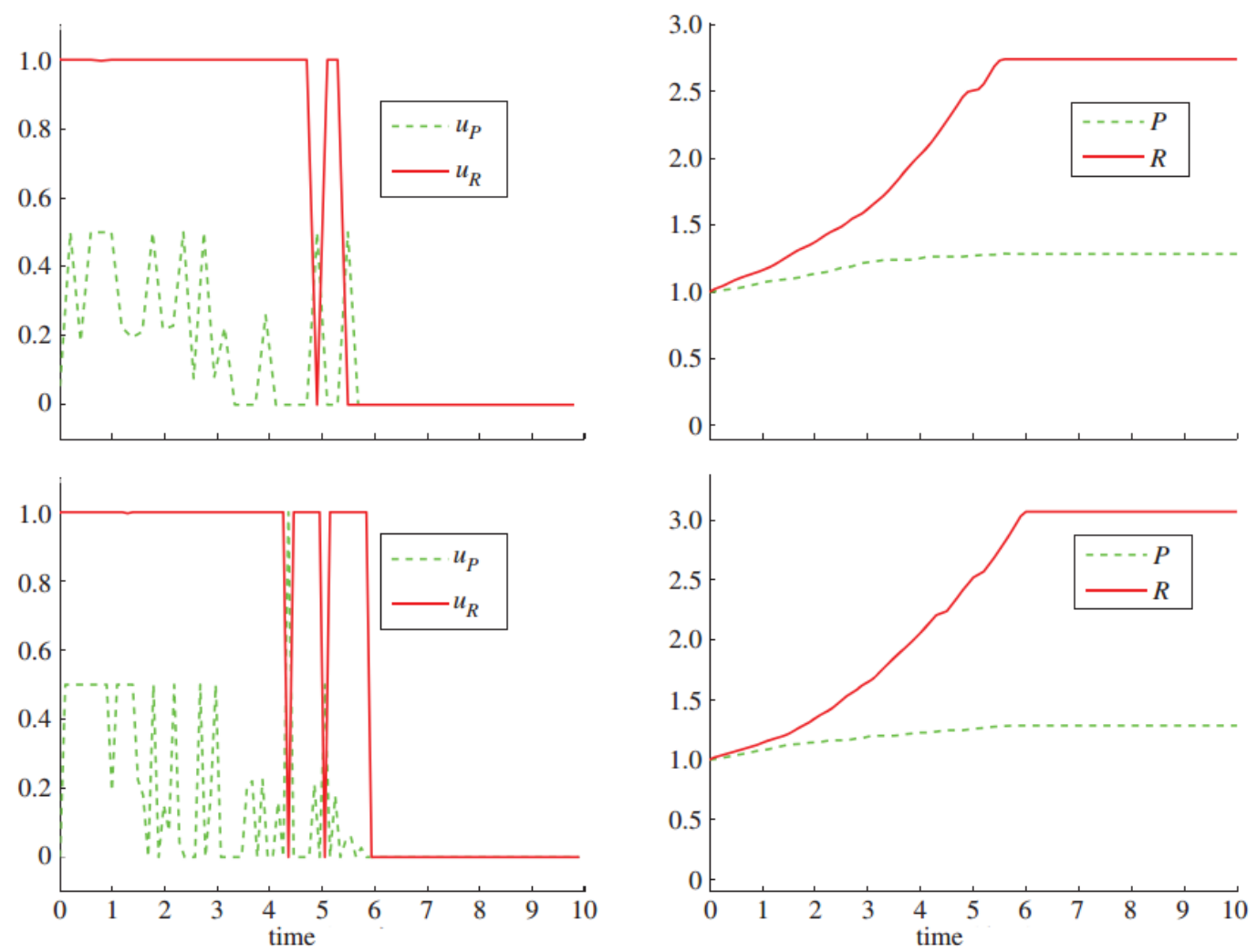

Figure 2.2: Numerical outcomes of the game on interval $[0,10]$ with continuous strategies: $\nu=100, \varsigma=50$ (top) and $\nu=100, \varsigma=100$ (bottom). Here $P(0)=R(0)=1$.

\subsection{Another way to model the problem}

Considering that energy level of mites depend on on their activities, another model including their energy dynamics was examined in Staňková et al. (2013a). This model assumes that the population change depends also on mean internal energy levels of the predator and the prey and that energy levels increase/decrease depending on feeding as well as on intrinsic energy consumption. While the model was first introduced and analyzed analytically in a very general form, some of the parameters were later fixed, based on the data from Sabelis and Overmeer (1987); Kroon et al. (2008). The remaining parameters, which are kept free are listed in Table 2.4. The dynamics of this model are given by:

\begin{tabular}{|l|l|}
\hline parameter & interpretation \\
\hline$\Gamma$ & length of the summer season \\
$R(t)$ & fruit-tree red spider mite (prey) population density at time $t \in[0, \Gamma]$ \\
$P(t)$ & predatory mite population density at time $t \in[0, \Gamma]$ \\
$E^{R}(t)$ & internal energy of the prey at time $t \in[0, \Gamma]$ \\
$E^{P}(t)$ & internal energy of the predatory mite at time $t \in[0, \Gamma]$ \\
$u^{R}(t)$ & the strategy of the prey population at time $t \in[0, \Gamma]$ \\
$u^{P}(t)$ & the strategy of the predatory population at time $t \in[0, \Gamma]$ \\
\hline
\end{tabular}

Table 2.4: Notation of the game-theoretical model with internal energy variables 

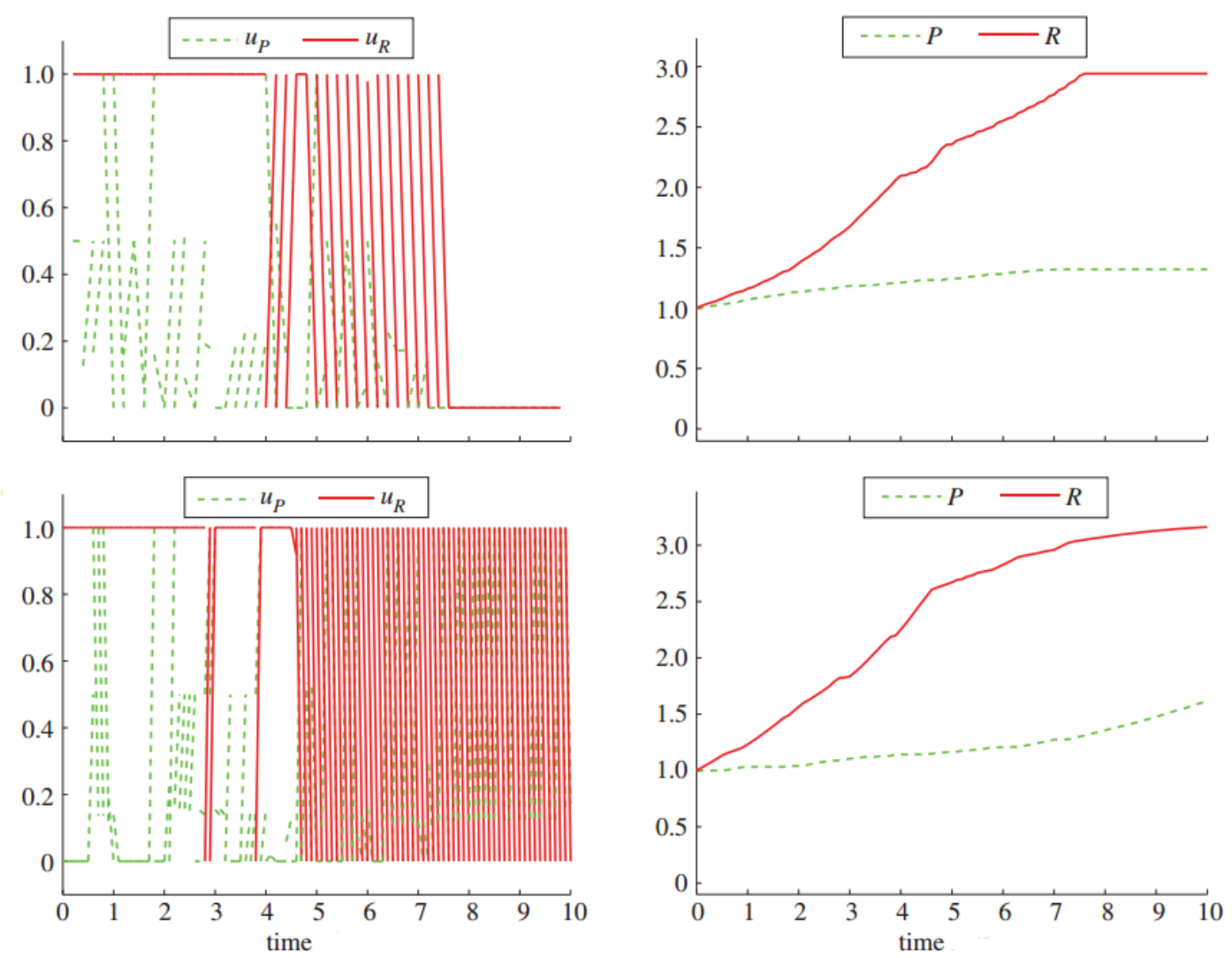

Figure 2.3: Numerical outcomes of the game on the interval $[0,10]$ with discontinuous strategies: $\nu=100, \varsigma=50$ (top) and $\nu=100, \varsigma=100$ (bottom). Here $P(0)=R(0)=1$.

$$
\begin{aligned}
\frac{\mathrm{d} E^{P}}{\mathrm{~d} t} & =-\frac{1}{250}\left(1-u^{P}\right) E^{P}+\psi u^{P} u^{R} R-\psi u^{P} E^{P} \\
\frac{\mathrm{d} E^{R}}{\mathrm{~d} t} & =-\frac{1}{250}\left(1-u^{R}\right) E^{R}+\psi u^{R}-\psi u^{R} E^{R}, \\
\frac{\mathrm{d} P}{\mathrm{~d} t} & =-\frac{1}{20} P+\frac{1}{5} \kappa u^{P} E^{P} P, \\
\frac{\mathrm{d} R}{\mathrm{~d} t} & =-\frac{1}{20} R+\frac{1}{5} u^{R} E^{R} R-\frac{1}{5} u^{P} u^{R} P R .
\end{aligned}
$$

The variables $E^{R}$ and $E^{P}$ represent the energy levels of the average predatory and the average prey individual, respectively, and parameters $\psi$ and $\kappa$ are kept free within the range $[0,1]$.

An optimal predator strategy $u^{P, *}(t) \in[0,1]$ for $t \in[0, \Gamma]$ satisfies

$$
u^{P, *}=\arg \sup _{u^{P}(\cdot)} P(\Gamma) .
$$

An optimal prey strategy $u^{R, *}(t) \in[0,1]$ for $t \in[0, \Gamma]$ satisfies

$$
u^{R, *}=\arg \sup _{u^{R}(\cdot)} \int_{0}^{\Gamma}\left(1-u^{R}(t)\right) E^{R}(t) R(t) \mathrm{d} t .
$$

Equations (2.55) and (2.56) are the fitness functions for the predatory mites and the fruittree red spider mites, respectively. The fitness function for the predatory mites (2.55) is 
$P(\Gamma)$ as in the previous model, reflecting the fact that all predatory individuals being alive at the end of the summer season (independently whether they are active or in diapause) have a chance to survive the winter. The fitness function for the prey mites (2.56) reflects the fact that only the prey individuals that are in diapause at the end of the summer season have a chance to survive the winter, while the longer in diapause they are and the more internal energy they have, the higher chance of survival they have.

In Staňková et al. (2013a), the problem of finding optimal strategies of both populations maximizing their fitness functions defined in (2.55) and (2.56), respectively, subject to the ODE system (2.51)-(2.54), was solved analytically for all feasible parameter values for $\psi$ and $\kappa$ (those assuring well-posssedness of the problem (2.51)-(2.56)) were found.

For all these feasible values of $\psi$ and $\kappa$ and depending on the length of the summer season $\Gamma$, the optimal strategy for the predatory mites is to stay active during the entire summer season, see Figure 2.4, while typically the prey which is all active at the beginning of the summer season starts entering diapause from a certain point in a monotonically decreasing fashion. Once all prey enters the diapause, it never comes back, i.e., its diapause process is irreversible. The optimal strategy for the prey is sketched in Figure 2.5. While in this figure the decrease in strategy is depicted as linear, in Staňková et al. (2013a,b) it was proven that the decrease in the optimal strategy to 0 is either monotonically decreasing or abrupt (then the optimal strategy is bang-bang (Olsder, 1997)). As the optimal strategy follows the principle of optimality (Bellman, 1957), the length of the season defines which of the strategy profiles from Figure 2.5 applies. Moreover, it was shown in Staňková et al. (2013b)

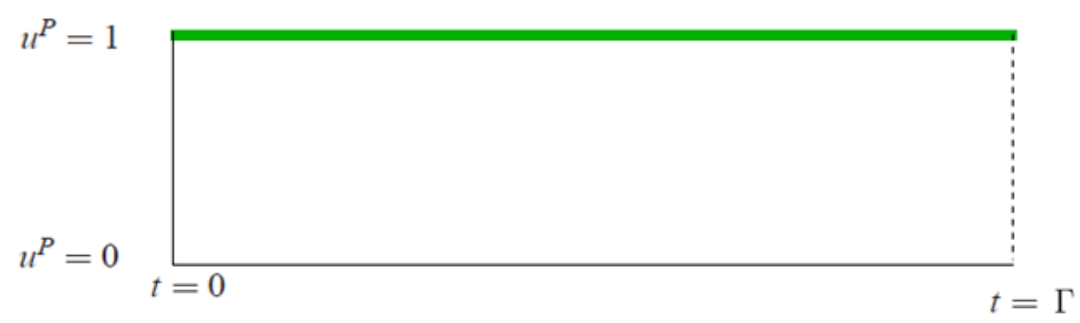

Figure 2.4: The optimal strategy for the predatory mites is to stay active during the entire summer season. Here $u^{P}$ indicates the optimal strategy (active ratio) for the predatory mites $\left(u^{P}(t) \in[0,1]\right.$ for each $\left.t \in[0, \Gamma]\right)$. This figure is taken from Staňková et al. (2013a).

that for a higher initial number of the predatory mites, the model predicts that the prey mites will start entering the diapause earlier, which matches the data. Therefore, we believe that a more complex model which has a higher number of state variables, including energy levels of a mean predator and prey individual, is needed to reflect the interactions in the predator-prey system of interest with a higher level of realism.

\subsection{Conclusion \& discussion}

The main conclusion of our analysis is that the very simple game-theoretical model we proposed to describe the interactions between the predatory mites and the fruit-tree red 


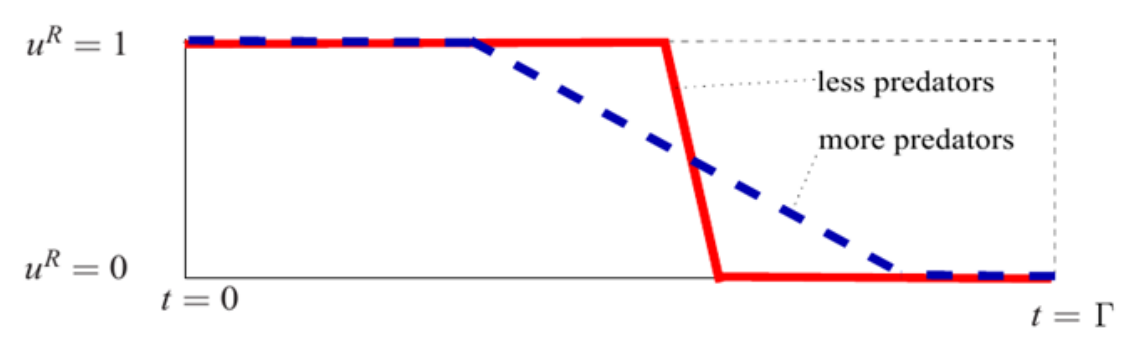

Figure 2.5: If the initial number of predatory mites increases, the prey mites begin to enter diapause earlier, but more gradually, balancing between having enough energy to survive the diapause and escaping predation. The solid line represents the strategy of the prey when facing less predators (i.e., smaller $P(t))$. The dashed line represents the strategy of the prey when facing more predators (i.e., bigger $P(t)$ ). Here $u^{R}$ indicates the optimal strategy (active ratio) for the prey $\left(u^{R}(t) \in[0,1]\right.$ for each $\left.t \in[0, \Gamma]\right)$. This sketch is taken from Staňková et al. (2013b).

spider mites produces strikingly different results than those the biologists observe.

As individuals in diapause cannot feed, the diapause should negatively influence their internal energy levels. Therefore, the models proposed in Staňková et al. (2013a,b) including additional state variables defined as energy storage for a mean predatory or prey mite, were introduced.

While the predicted optimal strategies calculated for the energy-enhanced models correspond to the laboratory and field observations very well - it is expected that the prey reacts to the presence of the predator by changing the manner and speed of its diapause induction, while the predatory mites have no real reason to enter diapause themselves, as there are no external threats to them present in the system, the initial model generates very different outcomes - the diapause of both the predatory mites and the fruit-tree red spider mites seems to be reversible, and moreover, the predators seem to react to the behavior of the prey to a larger extent than the prey reacts to the behavior of the predator (their frequent switches are found in our solutions), which contradicts the field observations.

In most field observations the predators indeed stay active for the entire season. However, in rare cases it might also happen that the predators enter and leave diapause during the season, while the diapause induction in the prey is irreversible. This is due to the fact that the diapause in predatory mites is much more flexible than that in the prey mites. In very recent and yet unpublished case studies, it is observed that in the laboratory conditions it is possible to achieve a situation when predatory mites enter diapause once the fruit-tree red spider mites do, if the predatory mites are exposed to the prey's odors sufficiently long. Subsequently, much less likely but possibly, the prey might become active when the predators are in diapause, followed by the predators becoming active as well. While repeatedly entering diapause/active state is an outcome of our game-theoretical model, the way in which the predator and the prey mites are predicted to enter the active/diapause state is too versatile and differs from our empirical observations. We conclude that the models including energy dynamics match the field and laboratory observations much better than the initial model 
that we proposed. The results in this chapter suggest that including energy dynamics is essential to the predictions of our specific case of predator-prey models and coincides with a common belief that including variables that describe the internal states of the interacting species is necessary to describe nontrivial predator-prey systems with a sufficient level of realism (McNamara and Houston, 1996; McNamara, 2001).

For all models we proposed so far we still have to find parameter domains for which the optimal strategies of the predatory and the prey populations, derived in this chapter, are resistant against invasion by mutant strategies. Under certain assumptions, such as that a rare mutant either minimizes the fitness of the resident population or has a similar same fitness function but with different characteristics, we should be able to find the parameter domains ensuring evolutionary stability of the outcomes relatively easily. Ultimately, we hope to explain multi-seasonal dynamics of the predatory mites and the fruit-tree red spider mites.

To calculate the optimal strategies for the model introduced in this chapter, we adopted an analytical approach with general parameter values first, followed by numerical calculations for their specific values. While the analytical calculations helped us to show that the predictions of the model do not coincide with laboratory and field observations, it was impossible for us to solve the problem by analytical means only. Numerical calculations gave us more understanding to the range of possible optimal strategies for the model, even for the same set of parameter values. In the following chapters, we will therefore confine ourselves to the numerical and simulation studies as the complexity of the problems which these chapters are dealing with is increasingly higher. 


\section{Discrete-space vs. continuous-space}

\section{Contents}

3.1 Introduction $\ldots \ldots \ldots \ldots \ldots \ldots \ldots$

3.2 The nonspatial game ....................... 49

3.3 Spatial games ....................... 50

3.3 .1 Game field . . . . . . . . . . . . . 50

3.3 .2 Spatial game rules . . . . . . . . . . . . . . . 51

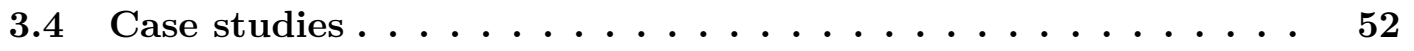

3.4 .1 The generalized RSP game . . . . . . . . . . . . 52

3.4 .2 Enhanced RSP game . . . . . . . . . . . . . . . . . 53

3.4 .3 Settings of the case studies $\ldots \ldots \ldots \ldots$. . . . . . . 54

3.4.4 Case study 1: Impact of the dispersal conflict . . . . . . . . . . 57

3.4.5 Case study 2: Impact of the interaction radius . . . . . . . . . . 61

3.4.6 Case study 3: Impact of the lifespan . . . . . . . . . . . . . 65

3.5 Conclusion \& discussion $\ldots \ldots \ldots \ldots \ldots$ 


\section{Abstract}

In spatial games it is usually assumed that individuals interact in spatially explicit structures in discrete or continuous space. Discrete and continuous models may differ in their assumptions on individuals' behaviors such as reproduction and dispersal and hence may lead to different frequency and population dynamics, even when describing the same biological problems. Typically, only discrete models or continuous models are selected when spatial extensions of (often known) nonspatial games are made. A thorough understanding of the difference between the two groups of spatial models is needed in order to decide when these two types of models can be interchanged and when only one of the two should be chosen. As a first step to such understanding, in this chapter we select one representative from each of the two groups of spatial models as a spatial extension of a nonspatial game. In case studies with enhanced rock-scissors-paper games, we examine and compare the dynamics of these two games and compare them with the replicator dynamics. If the studied nonspatial game has an ESS, its discrete-space and continuous-space frequency variants have either an attracting point or an attracting cycle. But often, in scenarios with different assumptions on individuals' reproduction, dispersal and lifespan, we observe that the predictions of the discrete and continuous models differ from each other and from the nonspatial dynamics, where the discrete-space model seems to have much higher variance in predictions per run when compared to the continuous-space model. In particular, the two types of spatial models generate strikingly different ecological dynamics when the space is saturated - the continuous-space game has a very high population size at saturation, while the discrete-space game has a much lower saturated population size, even when we impose the same density-dependence limits in the two games. Different ecological dynamics seem to lead to very different frequency dynamics, leading to very different predictions of the two types of spatial models. This calls for caution when selecting a spatial variant of a known nonspatial game.

Keywords: Spatial games, Discrete space, Continuous space, Replicator dynamics

Adapted from our article in preparation: You Li, Thuijsman Frank, Katharina Schüller, Uyttendaele Philippe, Staňková Kateřina. Discrete-space vs. continuous-space games, 2017. In preparation. 


\subsection{Introduction}

Spatially explicit evolutionary games (spatial games) are often perceived as a refinement of the nonspatial evolutionary games with more realistic assumptions on individuals' actions. While standard (evolutionary) game dynamics (such as replicator dynamics) assume that the population of interacting individuals is well mixed (and that a focal individual is allowed to interact with any individual in the population), the spatial games allow for making the interactions among individuals more or less localized and do not require the population to be well mixed.

However, the chosen spatial structure of the game field may be as important as players' strategies and fitness measures in determining its frequency and population dynamics. This structure often implies who is allowed to interact with whom. Commonly, individuals are assumed to interact in a predefined discrete space (Killingback and Doebeli, 1996; Kerr et al., 2002; Sysi-Aho et al., 2005) (discrete-space games) or, less commonly, in a continuous space (Lion and Baalen, 2008; Cardillo et al., 2012) (continuous-space games). The discrete-space games are often played over a grid composed of identical cells and individuals' positions and dispersal are therefore limited to this grid. Among these grid models, a square grid is the most common structure (Nowak and May, 1992; Durrett and Levin, 1997; Nakamaru et al., 1997; Szabó and Tőke, 1998; Durrett and Levin, 1998; Brauchli et al., 1999; Reichenbach et al., 2007; Noailly et al., 2009; Avelino et al., 2014), as opposed to grids composed of hexagonal or other cells (Okabe et al., 2009; Uyttendaele and Thuijsman, 2015; Abrudan et al., 2016). In continuous games, individuals are usually allowed to reside and disperse to at any position of the game field (Lion and Baalen, 2008; Cardillo et al., 2012; Gallaher and Anderson, 2013; Gallaher et al., 2017). In either case, spatial games are usually played over a predefined torus with periodic boundary conditions (also known as flat torus), to avoid complications with boundary conditions.

As opposed to spatial games, standard game dynamics do not take into account any type of the spatial structure. As such, standard game dynamics describe games that are nonspatial. In the remainder of this chapter, we may use the term "nonspatial games" for describing standard game dynamics.

While nonspatial games are usually mathematically tractable and very intuitive, the assumptions on individuals' interactions are often perceived as oversimplified. From that perspective, the spatial games represent a movement into more realistic models of real biological populations. However, there is not much research in how the frequency and population dynamics of different spatial games differ from each other and why. Moreover, if the nonspatial games are oversimplified, we need to understand why and when the prediction of specific spatial models coincide with or differ from those of the nonspatial games. Whether these predictions differ depends on the specific spatial game rules, such as local interaction ${ }^{13}$ (as opposed to a global interaction assumption in nonspatial games) (Durrett and Levin,

\footnotetext{
${ }^{13}$ Some researchers, such as Santos et al. (2012) use "structured population" to indicate that interactions between individuals taking place within neighborhoods, while "unstructured" would refer to global interactions.
} 
1998; Uyttendaele and Thuijsman, 2015), sequential updating (as opposed to a simultaneous updating rule in nonspatial games) (Nowak and May, 1993; Adami et al., 2016), birth and death mechanism (as opposed to nonspatial games where there are no birth and death assumptions) (Ohtsuki et al., 2007), etc.

To our best knowledge the comparison of the dynamics of discrete-space and continuousspace games is largely lacking. As the first step to bridge this gap we will focus on comparing two representative discrete-space and continuous-space games played over the torus with periodic boundary conditions. We will focus on their frequency and population dynamics, and compare both with those of the replicator dynamics (Hofbauer and Sigmund, 1998) as a typical representative of a nonspatial game.

The two spatial games and the nonspatial game that we will compare with each other are on purpose chosen as typical representatives of their groups: The discrete-space game will be the one with a square grid, played over the discrete torus. ${ }^{14}$ While in the simplest case of the grid games every cell hosts one individual and there are no empty cells in the game (Nowak and May, 1992; Durrett and Levin, 1997; Nakamaru et al., 1997; Szabó and Tőke, 1998; Brauchli et al., 1999; Noailly et al., 2009), we will allow for empty cells typically introduced in more advanced discrete-space models (Reichenbach et al., 2007; Avelino et al., 2014). These empty cells can be filled via dispersal of an individual (Kerr et al., 2002; Reichenbach et al., 2007) or its offspring (Godfrey Smith, 2009; Zietsch et al., 2014), while new empty cells are created when an individual moves or dies. Each focal individual in this game can interact only with other individuals in its neighborhood, which is defined as the cell occupied by the focal individual and its 8 neighboring cells.

Our continuous-space game is played over a torus with periodic boundary conditions defined as a proper subset of $\mathbb{R} \times \mathbb{R}$. Similarly as in the discrete game, the focal individual can interact only with other individuals from its neighborhood, here (in continuous space) defined as a disc around the focal individual with a prespecified radius. Unlike in the grid models, the individuals can move and place their offspring to any place within their neighborhood, while it follows from the continuity of the torus that the probability that two individuals reside at exactly the same coordinate at a given time is zero.

Our nonspatial game will be the standard replicator dynamics as a very common representative of nonspatial games in evolutionary game theory (Hofbauer and Sigmund, 1998).

When comparing the resulting frequency and population dynamics of our discrete-space and continuous-space games with each other and with those of the nonspatial game, the two spatial games will have the same interaction rules and thus the only difference is their game field and assumptions on the focal individuals' reproduction if there is no empty cell in its neighborhood - we will do experiment with 3 versions of the discrete game differing in such assumptions on reproduction. This is to see to what extent the assumptions related to the game field impact the resulting dynamics.

Conceptually, the continuous-space models seem to be more flexible when it comes to defining and adjusting the neighborhood and its structure. And it is obvious that for some

\footnotetext{
${ }^{14}$ In Chapter 4 we will focus on how predictions regarding coexistence of microbial strains differ when modeled as a game in a hexagonal and square grid.
} 
biological interactions, one model may be more suitable than other ones, especially if their predicted frequency and population dynamics differ (Nowak, 2006; Rand and Nowak, 2013). If, for example, the individuals can disperse and/or reproduce only when there is an available free space in a grid model while there is always a free space in a continuous-space model, then the differences in the dynamics are expected to occur especially when the space is saturated.

The reason why we include comparison of the spatial models with the replicator dynamics is that various assumptions of the spatial and nonspatial models may generate results close and far away from the replicator dynamics (Huang and Wu, 2012; Wang et al., 2016).

Usually, research on spatial-game models focuses either on their transient (Yearsley, 2004; You et al., 2017) or saturated (Adami et al., 2016; You et al., 2017) phases. The transient phase refers to a (still) growing population, whilst the saturated phase corresponds to a situation when population size does not change anymore, i.e., the space is saturated. Most studies focusing on spatial dynamics seek frequency and/or population equilibria which they consider stable (Noailly et al., 2009; Adami et al., 2016). Clearly, we may observe two types of the stable equilibria: the transient and the saturated stable equilibria where the term transient stable equilibrium refers to a short-term stability (Yearsley, 2004), while the saturated stable equilibrium refers to a long-term stability. In this chapter we will focus on the saturated phase, ${ }^{15}$ with which the nonspatial equilibrium frequencies and populations will be compared. The main goal is to understand how much and why the nonspatial, discrete-space, and continuous-space dynamics differ.

The rest of this chapter is composed as follows. In Section 3.2 we introduce the nonspatial dynamics. In Section 3.3 we introduce the spatial games defined in discrete and continuous space. In Section 3.4.3 we demonstrate case studies with these spatial games regarding individuals' behaviors such as reproduction and dispersal. In Section 3.5 we discuss the relations between the three kinds of dynamics, i.e., the nonspatial dynamics, the discretespace dynamics and the continuous-space dynamics, and finally conclude the chapter.

\subsection{The nonspatial game}

In this chapter, we will introduce our nonspatial game, the replicator dynamics (Hofbauer and Sigmund, 1998).

We assume that individuals of different types interact with each other. Let $T=\{1, \ldots, n\}$ be the set of types. Let $x_{i}, i \in T$, denote the frequency of the individuals of type $i \in T$ in the population. When a focal individual of type $i \in T$ interacts with an individual of type $j \in T$, the outcome is the number of offspring of type $i \in T$ that the focal individual will produce, $a_{i j}$. The number of offspring for all possible pairwise interactions can be organized

\footnotetext{
${ }^{15}$ In Chapter 5 we will study both transient and saturated frequency and population dynamics in a spatial game of cancer.
} 
in a fitness matrix $A$ :

$$
\left(\begin{array}{cccc}
a_{11} & a_{12} & \cdots & a_{1 n} \\
a_{21} & a_{22} & \cdots & a_{2 n} \\
\vdots & \vdots & \ddots & \vdots \\
a_{n 1} & a_{n 2} & \cdots & a_{n n}
\end{array}\right)
$$

The replicator dynamics (Hofbauer and Sigmund, 1998) define the time change $\dot{x}_{i}$ of frequency $x_{i}$ for each type $i \in T$ :

$$
\dot{x}_{i}=x_{i}\left(e_{i} A x^{\top}-x A x^{\top}\right),
$$

where $x=\left(x_{1}, \ldots, x_{n}\right)$ and $e_{i}$ are the frequency vector for all types and the $i$-th row of an $n \times n$ identity matrix, respectively. Even with the same fitness matrix, different initial conditions $\left(x(0)=x_{0}\right)$ may lead to different frequency (and population) dynamics. Replicator dynamics represent one of the dynamics which may have attractors coinciding with the Evolutionarily Stable Strategies (ESSs) of the game (3.1). An ESS is a strategy that, when used by the entire population, is immune against invasion by a small number of mutants playing a different strategy. An attractor corresponds to an ESS if it satisfies the evolutionary stability conditions introduced in Chapter 1. An attractor does not need to be an ESS, here we refer to a well-examined game (1.5) by Zeeman (1980); Bomze (1983); Hofbauer and Sigmund (1998).

\subsection{Spatial games}

\subsubsection{Game field}

Let $\Theta_{\mathbb{Z}}=\left\{(x, y) \mid(x, y) \in[0, L) \times[0, L)\right.$ and $\left.(x, y) \in \mathbb{Z}^{2}\right\}$ be a discrete-space torus with periodic boundary conditions, which will be the game field for the discrete-space game. In $\Theta_{\mathbb{Z}}$ the neighborhood of a focal individual is represented as a disc around this focal individual with a prespecified radius, see Figure 3.1. For the individual residing in the focal cell, its neighbors are the individuals within this neighborhood. The maximal number of individuals in a neighborhood is fixed, i.e., a neighborhood with radius 1 can host maximally 5 individuals, a neighborhood with radius 2 can host maximally 13 individuals, etc. (see Figure 3.1).

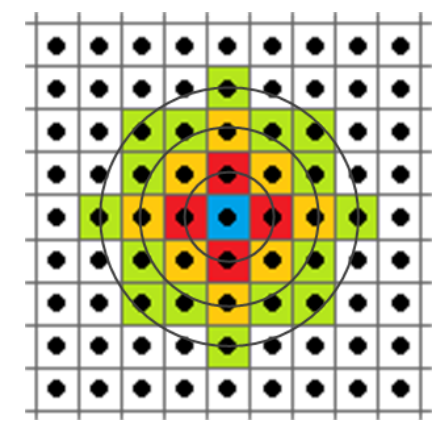

Figure 3.1: The neighborhood (in the discrete torus $\Theta_{\mathbb{Z}}$ ) which is an integer disc with radius $=1,2,3$. Each black dot represents one individual occupying one cell. 
Let $\Theta_{\mathbb{R}}=\left\{(x, y) \mid(x, y) \in[0, L) \times[0, L)\right.$ and $\left.(x, y) \in \mathbb{R}^{2}\right\}$, be a continuous-space torus with periodic boundary conditions, which will be the game field for the continuous-space game. In $\Theta_{\mathbb{R}}$ the neighborhood of a focal individual is a disc around the position of this individual with a prespecified radius. Theoretically the number of neighbors in a neighborhood (of any given radius) from $\Theta_{\mathbb{R}}$ is infinite.

\subsubsection{Spatial game rules}

Let us assume that the initial field is composed of individuals of types from set $T$. Initially, each type has the same number of individuals randomly distributed over the discrete-space torus $\Theta_{\mathbb{Z}}$ and the continuous-space torus $\Theta_{\mathbb{R}}$, respectively, so that the game field is not fully occupied (saturated).

For the spatial games we specify rules regarding individual density-dependent interactions, frequency-dependent interactions, reproduction and dispersal, which occur in generations: during one generation all individuals ${ }^{16}$ are selected as focal individuals, in a random order, to undergo the following actions:

Density-dependent interactions The focal individual checks whether its neighborhood is not saturated, otherwise it cannot reproduce. In the discrete-space torus $\Theta_{\mathbb{Z}}$, an individual's neighborhood always has a limit on the number of neighbors it can host, the so-called density-dependence limit (Figure 3.1). In $\Theta_{\mathbb{Z}}$ only if the number of neighbors is less than the density-dependence limit, the focal individual will proceed to further actions. We apply the same rule of density-dependent interactions to the continuous-space model, i.e., in a continuous neighborhood from $\Theta_{\mathbb{R}}$, if the number of neighbors is equal to or bigger than the density-dependence limit (determined by the discrete neighborhood with the same radius), the focal individual will not take further actions.

Frequency-dependent interactions The focal individual (assuming it is of type $i$ ) chooses a randomly selected neighbor to interact with (assuming it is of type $j$ ). If there are no neighbors in the neighborhood of the focal individual, no such frequency-dependent interaction takes place.

Reproduction After interaction with a randomly selected neighbor of type $j$, the focal individual of type $i$ will produce $a_{i j}$ individuals of its own type. Only after all individuals are selected as focal individuals, the offspring are placed in the game field.

Dispersal The offspring of all individuals are placed simultaneously at a randomly selected positions in the cells' neighborhoods after all focal individuals have completed their actions for the current generation. It may happen that two or more individuals are dispersing into

\footnotetext{
${ }^{16}$ While it may be more realistic for specific applications to assume that only a portion of individuals interact with others in each generation, here we assume that all living individuals are selected as focal individuals and hereby interact with others in each generation, in order to compare the resulting spatial dynamics with the replicator dynamics.
} 
the same $(x, y)$-coordinate in the discrete torus $\Theta_{\mathbb{Z}}$. We call such a situation a dispersal conflict. Such a conflict does not occur in the continuous-space game. Clearly, the dispersal conflict needs to be resolved somehow. We will consider 3 discrete-space game scenarios differing in the way how the dispersal conflict is handled:

- Scenario 1: The individuals injure each other and no one disperses into this $(x, y)$ coordinate (the "no placement" scenario).

- Scenario 2: Only one individual can occupy the $(x, y)$-coordinate, while this individual is selected randomly from all individuals who participate in the dispersal conflict (the "stochastic placement" scenario).

- Scenario 3: The type which has the highest count of individuals participating in the dispersal conflict fills this $(x, y)$-coordinate (the "deterministic placement" scenario). If there are more types with the highest count, the choice among them is random.

After the offspring of all focal individuals have been placed into the game field, all focal individuals (parents) are removed from the field. The next generation begins. Equivalently, all focal individuals (parents) have lifespan (determining the maximal number of generations that one individual can stay in the game field) as one generation.

The game ends after it is played for the prespecified number of generations. Clearly, we cannot run our spatial games for infinitely many generations. In this chapter we confine to the total of 10000 generations, as we consider this number of generations large enough to observe trends in the frequency and population dynamics in our games.

\subsection{Case studies}

To test how our discrete-space, continuous-space, and nonspatial games differ in terms of the frequency and population dynamics, we will run a number of case studies with a very well known class of games: the generalized Rock-Scissors-Paper (RSP) games (Durrett and Levin, 1998; Kerr et al., 2002; Czárán et al., 2002; Kirkup and Riley, 2004; Reichenbach et al., 2007; Avelino et al., 2014). The set of types is therefore $T=\{R, S, P\}$, where $R, S$ and $P$ stand for types Rock, Scissors and Paper, respectively.

\subsubsection{The generalized RSP game}

The generalized RSP game takes the following format (Weissing, 1991; Hofbauer and Sigmund, 1998):

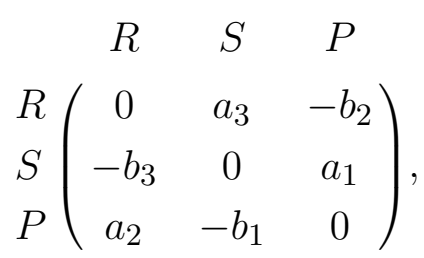

where $a_{1}, a_{2}, a_{3}, b_{1}, b_{2}, b_{3} \in \mathbb{R}$. We will first consider a specific case of the generalized RSP game, $A_{\varepsilon}$, described by Zeeman (1980), where $a_{1}=a_{2}=a_{3}=1+\varepsilon, b_{1}=b_{2}=b_{3}=1$ and 
$\varepsilon \in \mathbb{R}$. The fitness matrix $A_{\varepsilon}$ has then the following form:

$$
A_{\varepsilon}=\left(\begin{array}{ccc}
0 & 1+\varepsilon & -1 \\
-1 & 0 & 1+\varepsilon \\
1+\varepsilon & -1 & 0
\end{array}\right)
$$

There is a mixed-strategy Nash equilibrium $\left(\frac{1}{3}, \frac{1}{3}, \frac{1}{3}\right)$ for $A_{\varepsilon}$. As $\left(\frac{1}{3}, \frac{1}{3}, \frac{1}{3}\right)$ is a steady point of the replicator dynamics (3.2) for every $A_{\varepsilon}$, the frequency dynamics won't change if this point is set as an initial state. However, depending on the sign of $\varepsilon$, the Nash equilibrium $\left(\frac{1}{3}, \frac{1}{3}, \frac{1}{3}\right)$ has the different dynamic stability properties (Weissing, 1991; Hofbauer and Sigmund, 1998) (see also Section 1.3 from Chapter 1 ):

- If $\varepsilon>0$, the Nash equilibrium $\left(\frac{1}{3}, \frac{1}{3}, \frac{1}{3}\right)$ is an attractor of the replicator dynamics starting from an initial $x_{0}$ close to $\left(\frac{1}{3}, \frac{1}{3}, \frac{1}{3}\right)$, see Figure $3.2 \mathrm{a}$.

- If $\varepsilon=0$, the Nash equilibrium $\left(\frac{1}{3}, \frac{1}{3}, \frac{1}{3}\right)$ is neutrally stable and the replicator dynamics, starting from an initial $x_{0}$ close to $\left(\frac{1}{3}, \frac{1}{3}, \frac{1}{3}\right)$, forms a closed orbit (see Figure $3.2 \mathrm{~b}$ ).

- If $\varepsilon<0$, the Nash equilibrium $\left(\frac{1}{3}, \frac{1}{3}, \frac{1}{3}\right)$ is a repeller of the replicator dynamics starting from an initial $x_{0}$ close to $\left(\frac{1}{3}, \frac{1}{3}, \frac{1}{3}\right)$, where its trajectory moves to one of the three strategies $R, S$ or $P$, and stays there (see Figure 3.2c).

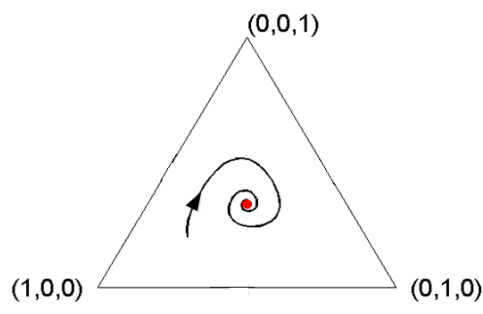

(a)

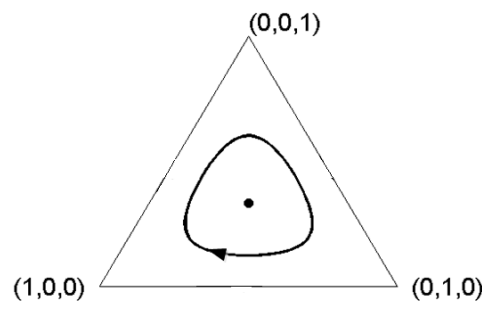

(b)

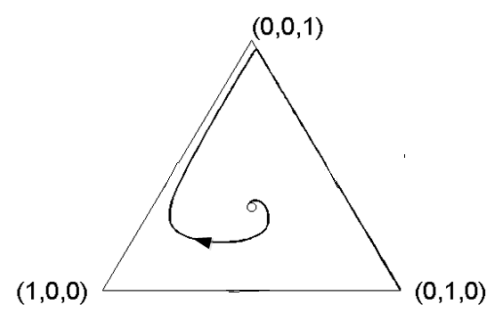

(c)

Figure 3.2: Trajectories of the nonspatial dynamics shown in the unit simplex with (a) $\varepsilon>0$ where the Nash equilibrium $\left(\frac{1}{3}, \frac{1}{3}, \frac{1}{3}\right)$ is an attractor (represented by a full red circle), (b) $\varepsilon=0$ where the Nash equilibrium $\left(\frac{1}{3}, \frac{1}{3}, \frac{1}{3}\right)$ is neutrally stable (represented by a full black circle), and (c) $\varepsilon<0$ where the Nash equilibrium $\left(\frac{1}{3}, \frac{1}{3}, \frac{1}{3}\right)$ is a repeller (represented by an empty circle). Each vertice corresponds to a frequency of 1 for the corresponding type.

Further, for the case when $\varepsilon>0$, the attractor is an ESS, whereas there is no ESS for the cases when $\varepsilon=0$ and $\varepsilon<0$ (Weissing, 1991; Hofbauer and Sigmund, 1998).

\subsubsection{Enhanced RSP game}

For the case studies we will consider a game derived from the generalized RSP game by adding a constant value to each element of its fitness matrix (enhanced RSP game). This is because we want that the fitness matrix represents the number of offspring and, therefore, 
it cannot have negative values. Starting from an $A_{\varepsilon}$ game with $\varepsilon=1, \varepsilon=0$ and $\varepsilon=-1$, we add 2 to each entry of the fitness matrices, which preserves the equilibrium properties of the corresponding $A_{\varepsilon}$ games. The obtained fitness matrices are:

$$
A_{1}^{+}=\left(\begin{array}{ccc}
2 & 4 & 1 \\
1 & 2 & 4 \\
4 & 1 & 2
\end{array}\right),
$$

with the Nash equilibrium $\left(\frac{1}{3}, \frac{1}{3}, \frac{1}{3}\right)$ being an ESS;

$$
A_{0}^{+}=\left(\begin{array}{ccc}
2 & 3 & 1 \\
1 & 2 & 3 \\
3 & 1 & 2
\end{array}\right),
$$

with the Nash equilibrium $\left(\frac{1}{3}, \frac{1}{3}, \frac{1}{3}\right)$ being neutrally stable;

$$
A_{-1}^{+}=\left(\begin{array}{lll}
2 & 2 & 1 \\
1 & 2 & 2 \\
2 & 1 & 2
\end{array}\right),
$$

with the Nash equilibrium $\left(\frac{1}{3}, \frac{1}{3}, \frac{1}{3}\right)$ being a repeller. We will refer to the games defined by matrices $A_{1}^{+}, A_{0}^{+}$and $A_{-1}^{+}$as to the enhanced RSP games $A_{\varepsilon}^{+}$.

\subsubsection{Settings of the case studies}

We let $L=100$. Initially, 999 individuals (333 individuals per type $i \in T=\{R, S, P\})$ are placed at randomly chosen $(x, y)$-coordinates in $\mathbb{Z}^{2}$ (without repetition) of $\Theta_{\mathbb{Z}}$ and $\Theta_{\mathbb{R}}$, respectively, thus we use the same initial placement of individuals, in both the discretespace and continuous-space games, for simulations in case studies.

Looking across all case studies within this section, we will examine the spatial dynamics for each $A_{\varepsilon}^{+}(\varepsilon \in\{1,0,-1\})$, for each model/scenario (i.e., the continuous-space model with no dispersal conflict, the discrete-spatial models with "no placement", "stochastic placement" and "deterministic placement" scenarios, respectively), for each interaction radius from $\{1,2,10,100\}$ and for each lifespan from $\{1,5\}$. Hence there are 96 matrix, model/scenario, radius and lifespan combinations. Due to the stochastic nature of spatial games, we repeat the simulation for each combination 15 times, as spatial dynamics of a single run may not demonstrate all possible outcomes of that combination. In the following case studies we demonstrate both the single-run and average outcomes. Beforehand we should introduce some concepts (see Section 3.4.3) that are used to examine the stability of spatial games.

\section{Stability concepts in spatial games}

To evaluate the spatial dynamics and for comparison to the nonspatial equilibria, we need stability concepts for spatial equilibria in terms of population size and frequencies of types. Here we need to point out that the population size at generation $t$ refers to the total number of individuals after including all offspring and removal of all parents once all interactions 
took place. We focus on the spatial stability at saturated population. In the following Definition 3.4.1, we use the term maximal total population, which refers to the largest total population of individuals reached over the run of the simulation.

Definition 3.4.1 (Saturated population). A population at generation $\tau$ is called saturated if the total number of individuals stays within $1 \%$ of the maximal population size in generations $\{\tau-499, \tau-498, \ldots, \tau\}$.

At a saturated population, we may observe that the spatial (frequency) dynamics is attracted to an attracting point or cycle, defined in Definitions 3.4.2 and 3.4.3, respectively:

Definition 3.4.2 (Attracting point). At saturation, we observe an attracting point $x^{*}=$ $\left(x_{1}^{*}, x_{2}^{*}, x_{3}^{*}\right)$ if there exists a generation $\tau$ so that $\left|x_{i}(t)-x_{i}(t+1)\right| \leq 0.001$ and $\left|x_{i}(t)-x_{i}^{*}\right| \leq$ 0.001 for each $i \in T$ and for all generations $t \in\{\tau-499, \tau-498, \ldots, \tau\}$.

Please note that we do not discuss the convergence speed of the frequency dynamics to the attracting point (in Definition 3.4.2) but find sufficient if we stayed very close to this point in the past 500 generations. The 500 generations were chosen because it is considered as a sufficiently large number of generations compared to the total number of 10000 generations per game considered here. Due to the nature of the spatial games it may be difficult to find the convergence speed to $x^{*}$ with a sufficient precision and as such, one may misinterpret an attracting behavior for another one.

Definition 3.4.3 (Attracting cycle). At saturation, we observe an attracting cycle if there exists a generation $\tau$ at least 500 generations before the last generation (i.e., the 10000th generation) so that for each $t>\tau,\left|x_{i}(t+\Delta)-x_{i}(t)\right| \leq 0.001$ for each $i \in T$ and for finite $\Delta>1$. We call $\Delta$ a period of the attracting cycle.

\section{Types of attracting points/cycles in spatial games}

Here we introduce types of attracting points/cycles that can be observed in single runs of our spatial games (including continuous-space model in every case study, discrete-space model and its three scenarios in every case study), to be able to classify the spatial (frequency) dynamics of the games considered in this chapter.

We categorize all our observed attracting points in spatial games as follows: The spatial (frequency) dynamics are attracted to

- the attracting point $x^{*}=\left(\frac{1}{3}, \frac{1}{3}, \frac{1}{3}\right.$ ) (see Figure 3.3 and 3.4 for frequency dynamics, of the same combination, achieved with different initial conditions);

- one of the three vertices $x^{*}=(1,0,0)$, or $x^{*}=(1,0,0)$, or $x^{*}=(1,0,0)$ (see Figure 3.5 as an example).

It is reasonable to set $x_{0}=\left(\frac{1}{3}, \frac{1}{3}, \frac{1}{3}\right)$ as an initial condition for the spatial games, although $x_{0}$ is a steady point for the corresponding nonspatial game (the replicator dynamics). Unlike the nonspatial dynamics which do not change and stay at $x^{*}=\left(\frac{1}{3}, \frac{1}{3}, \frac{1}{3}\right)$ when starting from 
this initial condition, the frequency dynamics of spatial games will always deviate from $x_{0}$ at early generations, due to the stochastic nature of the game rules. When $\left(\frac{1}{3}, \frac{1}{3}, \frac{1}{3}\right)$ is indeed an attractor of the frequency dynamics, then after the early generations, the frequency dynamics move back to it. This also applies for other off-boundary initial conditions. In Figure 3.4 we show an example where the frequency dynamics of the continuous-space game of $A_{1}^{+}$are attracted to the point $x^{*}=\left(\frac{1}{3}, \frac{1}{3}, \frac{1}{3}\right)$ with initial condition $(0.05,0.9,0.05)$.

It is useful to distinguish the two categories of attracting points, as they describe very different evolutionary outcomes regarding coexistence (here coexistence means that all types initially introduced in $\Theta_{\mathbb{Z}}$ and $\Theta_{\mathbb{R}}$ also stay in the field at saturation). For this reason, it may not be necessary to categorize the observed attracting cycles. For any attracting cycle we observe in these spatial games (including the continuous-space model in every case study, the discrete-space model and its three scenarios in every case study), its period $\Delta$ may vary in the interval $(10,500)$. Here, an example of an attracting cycle is shown in Figure 3.6, where the frequency dynamics are attracted to a cycle around the center $x=\left(\frac{1}{3}, \frac{1}{3}, \frac{1}{3}\right)$ with period $\Delta=241$.

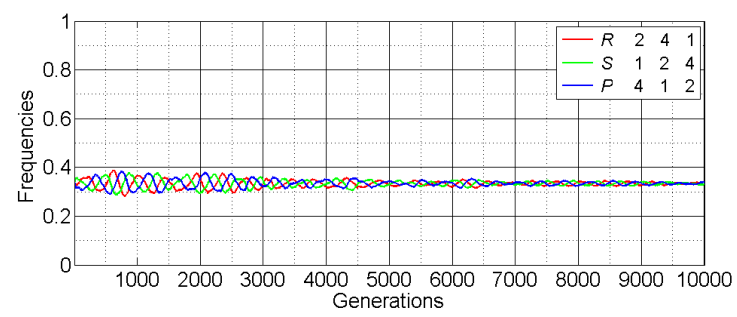

(a)

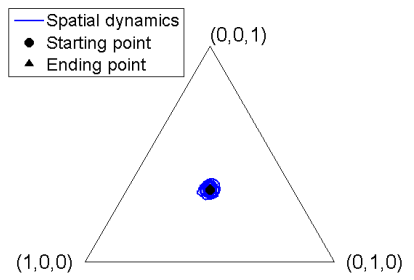

(b)

Figure 3.3: The frequency dynamics (of the continuous-space game for $A_{1}^{+}$with radius $=$ 2 , lifespan $=1$ ) are attracted to the center $x=\left(\frac{1}{3}, \frac{1}{3}, \frac{1}{3}\right)$ (a) after around 9200 generations, and such behavior is also shown (b) in the simplex. The frequency dynamics start with $x_{0}=\left(\frac{1}{3}, \frac{1}{3}, \frac{1}{3}\right)$.

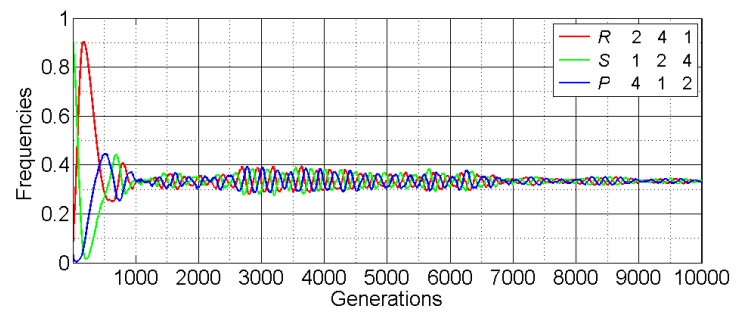

(a)

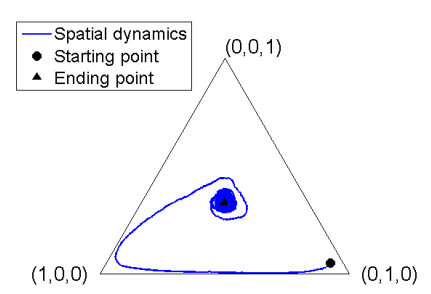

(b)

Figure 3.4: The frequency dynamics (of the continuous-space game for $A_{1}^{+}$with radius $=$ 2 , lifespan $=1$ ) are attracted to the center $x=\left(\frac{1}{3}, \frac{1}{3}, \frac{1}{3}\right)$ (a) after around 8500 generations, and such behavior is also shown (b) in the simplex. The frequency dynamics start with $x_{0}=(0.05,0.9,0.05)$. 


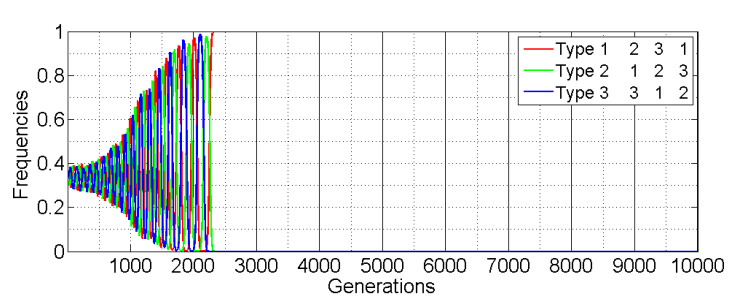

(a)

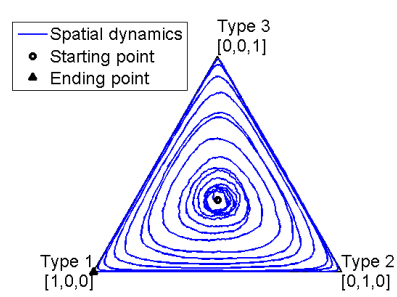

(b)

Figure 3.5: The frequency dynamics (of the discrete-space game for $A_{0}^{+}$with radius $=10$, lifespan $=1$, in the "no placement" scenario) are attracted to one of the three vertices $x=(1,0,0)$ (a) after around 3000 generations, and such behavior is also shown (b) in the simplex. The frequency dynamics start with $x_{0}=\left(\frac{1}{3}, \frac{1}{3}, \frac{1}{3}\right)$.

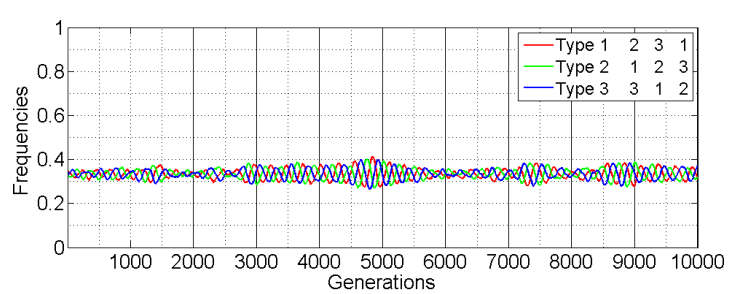

(a)

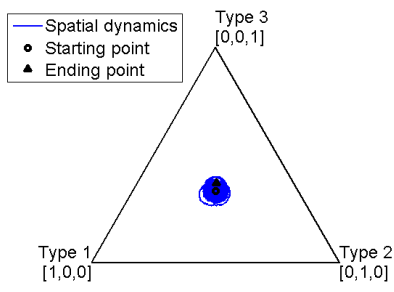

(b)

Figure 3.6: The frequency dynamics (of the discrete-space game for $A_{0}^{+}$with radius $=2$, lifespan $=1$, in the "stochastic placement" scenario) are attracted to a cycle (a) after around 8500 generations with $\Delta=241$, and such behavior is also shown (b) in the simplex. The frequency dynamics start with $x_{0}=\left(\frac{1}{3}, \frac{1}{3}, \frac{1}{3}\right)$.

\subsubsection{Case study 1: Impact of the dispersal conflict}

This case study focuses on the impact of dispersal conflict, by comparing the continuousspace dynamics (which has no dispersal conflict) and the discrete-space dynamics with "no placement" scenario, with "stochastic placement" scenario and with "deterministic placement" scenario. We let the radius be equal to 2, thus an individual can only undergo frequency-dependence interactions and reproduction if this individual's neighborhood currently hosts less than 13 individuals including itself. We keep the lifespan of individuals as one generation.

We examine the continuous-space dynamics for each of the three combinations of $A_{\varepsilon}^{+}$ $(\varepsilon \in\{1,0,-1\})$. We examine the discrete-space dynamics for each $A_{\varepsilon}^{+}$and each of the three dispersal conflict scenarios, thus for 9 matrix and scenario combinations. For each of the total of 12 spatial game combinations, we run simulations for 10000 generations and we repeat each of them 15 times, for the same initial field. We track both the population and frequency dynamics in each single run and then average those over the 15 runs. We compare the frequency dynamics generated by the spatial games to the nonspatial dynamics introduced in Section 3.4.1. 


\section{Population dynamics}

At saturation, the population size (averaged over 15 runs) with respect to each of the 12 (spatial model, matrix, scenario) combinations is shown in Table 3.1. Here we do not report the population size for every single run, as the variance of the 15 runs per combination is very small $(<1 \%$ of the averaged population size of this corresponding combination shown in Table 3.1). For the discrete-space games we measure an average number of dispersal conflicts per generation, averaged over 15 repetitions of the game times its 10000 generations. In Table 3.2 we show the results for each of the 9 matrix and scenario combinations. Here we do not report the mean number of dispersal conflicts for every single run, as the variance of the 15 runs per combination is very small $(<1 \%$ of the averaged number of dispersal conflict of this corresponding combination in Table 3.2). Looking across different models and scenarios

\begin{tabular}{l|l|l|l|l}
$\#$ & Continuous & $\begin{array}{l}\text { Discrete } \\
\text { no } \\
\text { placement }\end{array}$ & $\begin{array}{l}\text { Discrete } \\
\text { stochastic } \\
\text { placement }\end{array}$ & $\begin{array}{l}\text { Discrete } \\
\text { deterministic } \\
\text { placement }\end{array}$ \\
\hline$A_{1}^{+}$ & 10493.4 & 2080.4 & 4523.7 & 4495.1 \\
\hline$A_{0}^{+}$ & 10523.4 & 2049.8 & 4445.5 & 4435.8 \\
\hline$A_{-1}^{+}$ & 10536.9 & 2077.6 & 4500.2 & 4517.0
\end{tabular}

Table 3.1: The population size at saturation (averaged over 15 runs) given the 12 (matrix and scenario) combinations.

\begin{tabular}{l|l|l|l}
$\#$ & No placement & Stochastic placement & Deterministic placement \\
\hline$A_{1}^{+}$ & 876.2 & 2837.2 & 2860.9 \\
\hline$A_{0}^{+}$ & 847.8 & 2643.3 & 2646.7 \\
\hline$A_{-1}^{+}$ & 829.7 & 2551.5 & 2554.4
\end{tabular}

Table 3.2: Average number of dispersal conflicts per generation for the 9 (matrix and scenario) combinations of the discrete-space model, averaged over 15 runs of the game times its 10000 generations.

(different columns in Table 3.1), we observe very different population sizes at saturation for each of the $A_{\varepsilon}^{+}$matrices $(\varepsilon \in\{1,0,-1\})$ : the continuous-space model allows a high population size at saturation, i.e., its total population generally goes above 10000 . The discrete-space model allows only a low population size at saturation: its total population is around 4500 at saturation with "stochastic placement" and "deterministic placement" scenarios, while its total population goes to only around 2000 individuals at saturation with the "no placement" scenario. We observe that the dispersal conflict occurs in all three scenarios of the discretespace game (see Table 3.2). As expected, for a given $A_{\varepsilon}^{+}(\varepsilon \in\{1,0,-1\})$, the dispersal conflict occurs much more often with scenarios of "stochastic placement" and "deterministic placement" (2500 to 3000 times) than with the scenario of "no placement" (800 to 900 times). The individuals that cannot be placed in the discrete space due to the dispersal conflict (even if the density-dependence limit permits) are just added to the field in the continuous-space game. Moreover, we see that the frequency of the dispersal conflict is positively correlated 
with the population size at saturation in the discrete-space game, where less than $50 \%(x, y)$ coordinates of the discrete torus $\Theta_{\mathbb{Z}}$ are occupied at saturation (see Tables 3.1 and 3.2): A relatively high population leads to higher reproduction rate, causing a more frequent dispersal conflict.

\section{Frequency dynamics}

At saturation, the observed frequency dynamics (averaged over 15 runs) of $A_{\varepsilon}^{+}$for $\varepsilon=1,0$ and -1 are reported in Figures 3.7, 3.8 and 3.9, respectively. For the spatial frequency

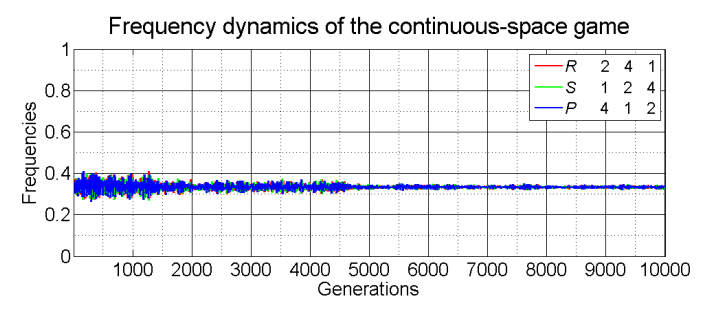

(a)

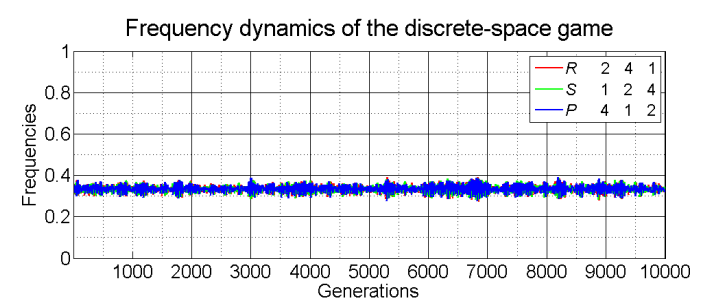

(c)

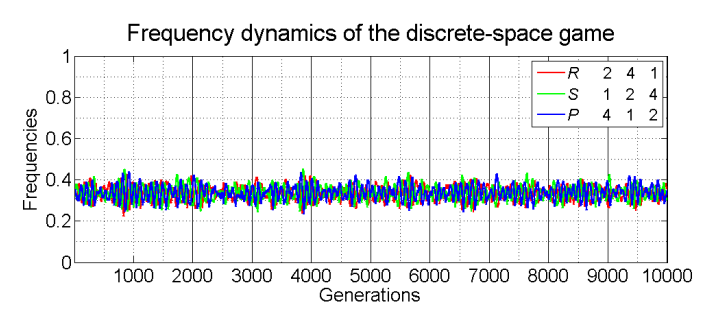

(b)

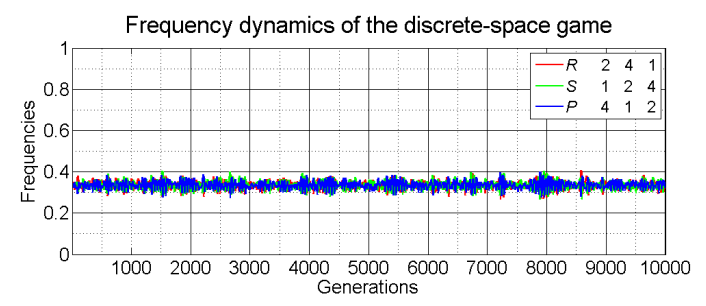

(d)

Figure 3.7: The frequency dynamics of $A_{1}^{+}$in (a) the continuous space, (b) the discrete space with "no placement" scenario, (c) the discrete space with "stochastic placement" scenario and $(\mathrm{d})$ the discrete space with "deterministic placement" scenario. Each subfigure shows frequency dynamics averaged over 15 runs of 10000 generations each. Radius is equal to 2.

dynamics (averaged over 15 runs) we may observe an attracting point $x^{*}=\left(\frac{1}{3}, \frac{1}{3}, \frac{1}{3}\right)$ or an attracting cycle. For $A_{1}^{+}$we observe its continuous-space dynamics are attracted to $x^{*}=\left(\frac{1}{3}, \frac{1}{3}, \frac{1}{3}\right)$ which is an ESS for the corresponding replicator dynamics, while the corresponding discretespace dynamics (of all three scenarios) differ from the continuous-space ones as they have attracting cycles (see Figures 3.7b-3.7d). For matrices $A_{0}^{+}$and $A_{-1}^{+}$(which do not have ESSs), their continuous-space dynamics are attracted to cycles (see Figures 3.8 and 3.9), while their discrete-space dynamics (of all three scenarios) are either attracted to cycles or to $x^{*}=\left(\frac{1}{3}, \frac{1}{3}, \frac{1}{3}\right)$, an attracting point which is not observed in terms of single runs, see Figures 3.8 and 3.9. If we look at dynamics averaged over 15 runs with matrices $A_{0}^{+}$and $A_{-1}^{+}$, the two spatial games lead to a more stable dynamical behavior than the nonspatial ones: While the nonspatial dynamics starting close to $\left(\frac{1}{3}, \frac{1}{3}, \frac{1}{3}\right)$ are either forming a closed orbit (in the game of $A_{0}^{+}$) or are repelled from this point (in the game of $A_{-1}^{+}$), the spatial dynamics have attracting cycles (which are observed in the games of $A_{0}^{+}$and $A_{-1}^{+}$) or are attracted to a point (observed in the game of $A_{-1}^{+}$). However, this conclusion does not hold for each single run. When we look on the discrete-space game with matrix $A_{-1}^{+}$and "no 


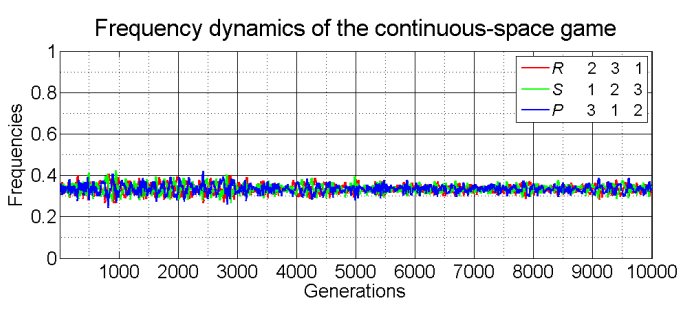

(a)

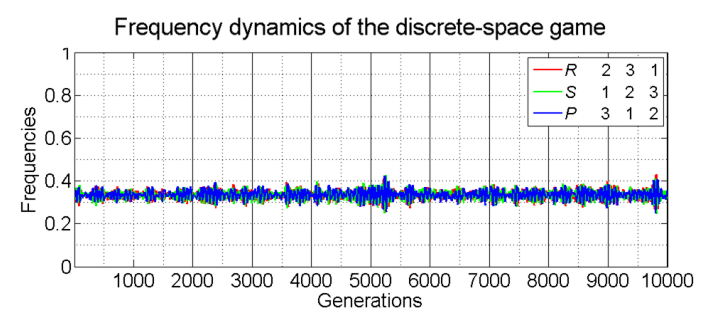

(c)

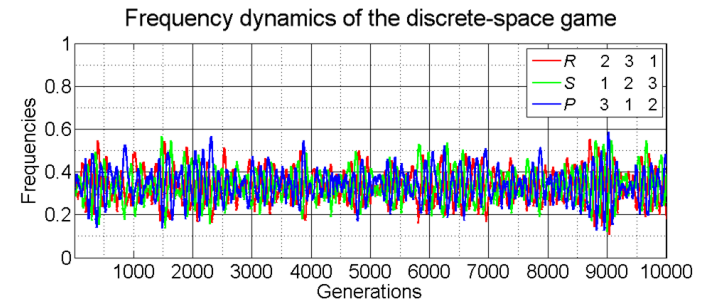

(b)

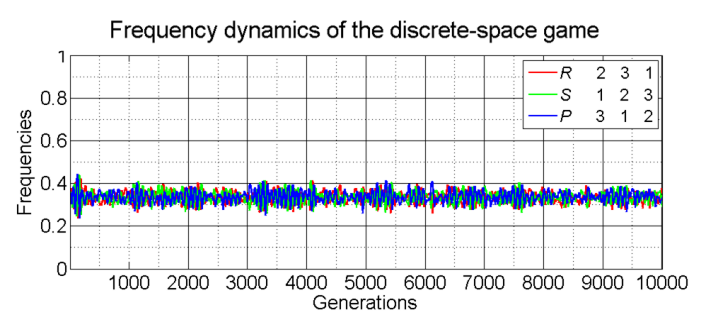

(d)

Figure 3.8: The frequency dynamics of $A_{0}^{+}$in (a) the continuous space, (b) the discrete space with "no placement" scenario, (c) the discrete space with "stochastic placement" scenario and (d) the discrete space with "deterministic placement" scenario. Each subfigure shows frequency dynamics averaged over 15 runs after 10000 generations. Radius is equal to 2 .

placement" scenario in each run, we observe that the discrete-space frequency dynamics per run move away from the initial condition $\left(\frac{1}{3}, \frac{1}{3}, \frac{1}{3}\right)$ to a population composed of only one of the types from $T=\{R, S, P\}$, while it is equally likely that this type is $R, S$, or $P$. We will call this phenomenon of only one type surviving (a different one per run) "random survival" throughout this chapter. The discrete-space model seems to have much higher variance in predictions per run when compared to the continuous model. Such random survival is not observed per run of continuous-space game $A_{\varepsilon}^{+}$for every $\varepsilon$.

In the remainder of this chapter, we will omit the report of averaged outcomes for any (matrix, model/scenario, radius, lifespan) combination whose single-run spatial dynamics show the random survival outcome, as in such case, the averaged outcomes do not report the spatial dynamics of a spatial game.

Based on our observations on the population and frequency dynamics (see Figures 3.7, 3.8 and 3.9), the impact of dispersal conflict is summarized as follows:

- The dispersal conflict may cause the population and frequency dynamics of discretespace games (including all scenarios) differ from those of continuous-space games, for every $A_{\varepsilon}^{+}(\varepsilon \in\{1,0,-1\})$.

- In the discrete-space game, the population and frequency dynamics of the "stochastic placement" and the "deterministic placement" scenarios after 10000 generations coincide with each other for every $A_{\varepsilon}^{+}(\varepsilon \in\{1,0,-1\})$. This is to be expected as they basically have very similar frequency-dependence rule.

- Compared to the two scenarios ("stochastic placement" and "deterministic placement") which allow placement of offspring, the "no placement" scenario can result in very 


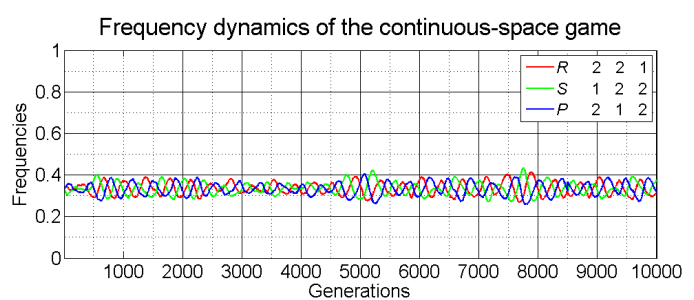

(a)

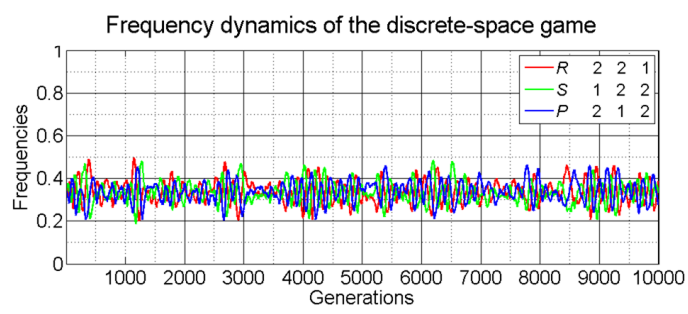

(c)

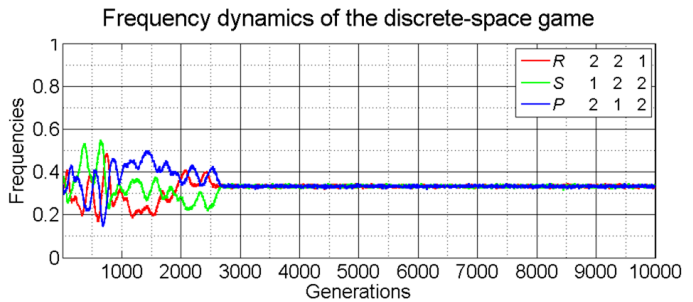

(b)

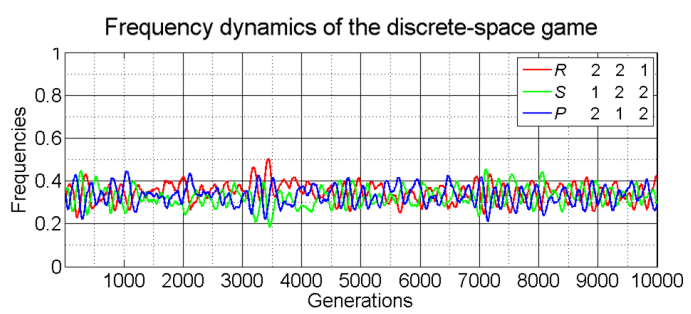

(d)

Figure 3.9: The frequency dynamics of $A_{-1}^{+}$in (a) the continuous space, (b) the discrete space with "no placement" scenario, (c) the discrete space with "stochastic placement" scenario and (d) the discrete space with "deterministic placement" scenario. Each subfigure shows frequency dynamics averaged over 15 runs after 10000 generations. Radius is equal to 2 .

different population and frequency dynamics. The discrete frequency dynamics of the former two scenarios correspond to the continuous ones, and to the nonspatial ones, more often than those of the latter scenario.

\subsubsection{Case study 2: Impact of the interaction radius}

This case study focuses on the impact of different interaction radii on the dynamics. We set the interaction radius to 1, 2, 10 and 100, and the density-dependent limit to 5, 13, 317 and 10000, respectively. We fix the lifespan to one generation.

We examine the discrete-space dynamics for each $A_{\varepsilon}^{+}(\varepsilon \in\{1,0,-1\})$, each of the three dispersal conflict scenarios, and each radius from $\{1,2,10,100\}$. There are 36 (matrix, model/scenario and radius) combinations for the discrete-space game. We also examine the continuous-space dynamics for each $A_{\varepsilon}^{+}(\varepsilon \in\{1,0,-1\})$ and each radius from $\{1,2,10,100\}$. There are 12 (matrix and radius) combinations for the continuous-space model. For each of the 48 (matrix, model/scenario and radius) spatial games, we track the population dynamics as well as the frequency dynamics. For each combination we run 15 simulations with 10000 generations. The resulting dynamics are compared with each other and with the nonspatial game introduced in Section 3.4.1.

\section{Population dynamics}

At saturation, the population size (averaged over 15 runs) with respect to each of the 48 (matrix, model/scenario and radius) combinations is shown in Table 3.3. Here we do not report the population size for every single run, as the variance of the 15 runs per combination 
is observed small $(<1 \%$ of the averaged population size of this corresponding combination from Table 3.3). We observe that either the population goes extinct or it gets saturated.

\begin{tabular}{|c|c|c|c|c|c|}
\hline$\#$ & Radii & Continuous & $\begin{array}{l}\text { Discrete } \\
\text { no } \\
\text { placement }\end{array}$ & $\begin{array}{l}\text { Discrete } \\
\text { stochastic } \\
\text { placement }\end{array}$ & $\begin{array}{l}\text { Discrete } \\
\text { deterministic } \\
\text { placement }\end{array}$ \\
\hline \multirow{4}{*}{$A_{1}^{+}$} & 1 & 15710.6 & - & - & - \\
\hline & 2 & 11674.2 & 2649.6 & 4596.3 & 4538.7 \\
\hline & 10 & 10493.4 & 2080.4 & 4523.7 & 4495.1 \\
\hline & 100 & - & 2568.5 & 4427.7 & 4431.3 \\
\hline \multirow{4}{*}{$A_{0}^{+}$} & 1 & 15632.0 & - & - & - \\
\hline & 2 & 11364.1 & 2686.2 & 4456.7 & 4444.9 \\
\hline & 10 & 10523.4 & 2049.8 & 4445.5 & 4435.8 \\
\hline & 100 & - & 2575.9 & 4429.1 & 4420.7 \\
\hline \multirow{4}{*}{$A_{-1}^{+}$} & 1 & 15503.4 & - & - & - \\
\hline & 2 & 11533.2 & 2679.3 & 4503.3 & 4604.6 \\
\hline & 10 & 10536.9 & 2077.6 & 4500.2 & 4517.0 \\
\hline & 100 & - & 2578.5 & 4434.0 & 4431.0 \\
\hline
\end{tabular}

Table 3.3: The population size at saturation (averaged over 15 runs) given 48 different matrix, model/scenario and radius combinations. The symbol " - " represents extinction of a population, hence in the particular combination no saturated population is observed.

The extinction happens (i) when the radius is very small in the discrete-space model (for all scenarios with the radius 1 ), and (ii) when the interaction radius is at its largest value (= 100) in the continuous-space model. If the interaction radius is very small in the discretespace game and individuals are randomly scattered in the game field, individuals may not find any neighbor to interact with and their total number may drop. In all our spatial games, we observe that the total population experiences a decline in early generations. However, afterwards, the total population increases and avoids extinction in the continuous-space game, while this is not the case in the discrete-space game. To explain, let us consider the following situation: Imagine a neighborhood (with radius $=1$ ) hosting 4 individuals at generation $t$. Let us call this neighborhood $U_{\Theta_{\mathbb{Z}}}$ if the game is a discrete-space game and $U_{\Theta_{\mathbb{R}}}$ if it is a continuous-space game. If the density-dependence permits, one offspring is allowed to be placed in $U_{\Theta_{\mathbb{Z}}}$ (as the density-dependence limit is 5 for radius equal to 1 ) and multiple offspring are allowed to be placed in $U_{\Theta_{\mathbb{R}}}$. The number of individuals that $U_{\Theta_{\mathbb{Z}}}$ and $U_{\Theta_{\mathbb{R}}}$ hold would deviate from each other at generation $t+1$ : After all parents are removed from the game field, $U_{\Theta_{\mathbb{Z}}}$ holds zero or one individual, and this only individual may not be able to meet a neighbor for interaction and reproduction. The situation is different in $U_{\Theta_{\mathbb{R}}}$, which is more likely to hold more than one individuals and these individuals can interact with each other.

When the interaction radius is 100 in the continuous-space model, the density-dependent interactions are global. As said earlier, the total population at generation $t$, refers to the population after including all offspring and removal of all parents from generation $t$. Hence, the counted population at a given generation $t$ is composed of offspring. In continuous space, the number of offspring may go above the (global) density-dependence limit, causing 
a situation where no individual can reproduce before being removed from the field. As no offspring are then produced, the population goes extinct at generation $t+1$.

In ecology and evolution, studying extinction is as important as studying saturation. At saturation, the continuous-space game allows for higher population size than the discretespace game. Moreover, the size of the saturated population is dependent on the interaction radius in the continuous-space game, as the standard deviation between the population sizes at saturation with different radii (of every $A_{\varepsilon}^{+}$) is large (e.g., with $A_{\varepsilon}^{+}(\varepsilon \in\{1,0,-1\}$ ) the standard deviation of the averaged saturated population sizes given the radii $1,2,10$ and 100 is around $2.0 \times 10^{3}$ ). However, the size of the saturated population is scenariodependent in the discrete-space model and the standard deviation between the population sizes under different scenarios (of every $A_{\varepsilon}^{+}$) is large (e.g., for an $A_{\varepsilon}^{+}(\varepsilon \in\{1,0,-1\}$ ) the standard deviation of the averaged saturated population sizes given the three scenarios with a given radius is $10^{3}$.)

\section{Frequency dynamics}

At saturation, the observed frequency dynamics (averaged over 15 runs) are reported in Table 3.4. In Table 3.5 we report the observed frequency dynamics for a single run per combination. The results are compared to the replicator dynamics introduced in Section 3.4.1.

\begin{tabular}{|c|c|c|c|c|c|}
\hline \# & Radii & Continuous & $\begin{array}{l}\text { Discrete } \\
\text { no } \\
\text { placement }\end{array}$ & $\begin{array}{l}\text { Discrete } \\
\text { stochastic } \\
\text { placement }\end{array}$ & $\begin{array}{l}\text { Discrete } \\
\text { deterministic } \\
\text { placement }\end{array}$ \\
\hline \multirow{4}{*}{$A_{1}^{+}$} & 1 & $\mathrm{AP}-\left(\frac{1}{3}, \frac{1}{3}, \frac{1}{3}\right)$ & - & - & - \\
\hline & 2 & $\mathrm{AP}-\left(\frac{1}{3}, \frac{1}{3}, \frac{1}{3}\right)$ & AP- $\left(\frac{1}{3}, \frac{1}{3}, \frac{1}{3}\right)$ & $\mathrm{AP}-\left(\frac{1}{3}, \frac{1}{3}, \frac{1}{3}\right)$ & AP- $\left(\frac{1}{3}, \frac{1}{3}, \frac{1}{3}\right)$ \\
\hline & 10 & $\mathrm{AP}-\left(\frac{1}{3}, \frac{1}{3}, \frac{1}{3}\right)$ & AP- $\left(\frac{1}{3}, \frac{1}{3}, \frac{1}{3}\right)$ & AP- $\left(\frac{1}{3}, \frac{1}{3}, \frac{1}{3}\right)$ & $\mathrm{AP}-\left(\frac{1}{3}, \frac{1}{3}, \frac{1}{3}\right)$ \\
\hline & 100 & - & AP- $\left(\frac{1}{3}, \frac{1}{3}, \frac{1}{3}\right)$ & AP- $\left(\frac{1}{3}, \frac{1}{3}, \frac{1}{3}\right)$ & AP- $\left(\frac{1}{3}, \frac{1}{3}, \frac{1}{3}\right)$ \\
\hline \multirow{4}{*}{$A_{0}^{+}$} & 1 & $\mathrm{AC}$ & - & - & - \\
\hline & 2 & $\mathrm{AC}$ & $\mathrm{AC}$ & $\mathrm{AC}$ & $\mathrm{AC}$ \\
\hline & 10 & $\mathrm{AC}$ & RS & RS & $\mathrm{RS}$ \\
\hline & 100 & - & RS & RS & $\mathrm{RS}$ \\
\hline \multirow{4}{*}{$A_{-1}^{+}$} & 1 & $\mathrm{AC}$ & - & - & - \\
\hline & 2 & $\mathrm{AC}$ & RS & $\mathrm{AC}$ & $\mathrm{AC}$ \\
\hline & 10 & $\mathrm{AC}$ & RS & RS & $\mathrm{RS}$ \\
\hline & 100 & - & RS & RS & $\mathrm{RS}$ \\
\hline
\end{tabular}

Table 3.4: The frequency dynamics given 48 different matrix, model/scenario and radius combinations. Here we describe the averaged frequency dynamics (over 15 runs) as the evolutionary outcome per combination. The symbol "-" means no spatial dynamics are observed as the total population becomes zero. The symbols "AP- $\left(\frac{1}{3}, \frac{1}{3}, \frac{1}{3}\right)$ " mean that the frequency dynamics are attracted to $\left(\frac{1}{3}, \frac{1}{3}, \frac{1}{3}\right)$, the symbol "AC" means an attracting cycle is observed, and the symbol "RS" indicates that random survival occurs in at least one out of 15 runs.

Our observations are summarized as follows:

- (Continuous-space dynamics vs. discrete-space dynamics) The discrete-space dynamics 


\begin{tabular}{|c|c|c|c|c|c|}
\hline$\#$ & Radii & Continuous & $\begin{array}{l}\text { Discrete } \\
\text { no } \\
\text { placement }\end{array}$ & $\begin{array}{l}\text { Discrete } \\
\text { stochastic } \\
\text { placement }\end{array}$ & $\begin{array}{l}\text { Discrete } \\
\text { deterministic } \\
\text { placement }\end{array}$ \\
\hline \multirow[t]{3}{*}{$A_{1}^{+}$} & $\begin{array}{l}1 \\
2 \\
10 \\
100 \\
\end{array}$ & $\begin{array}{l}\text { AP- }\left(\frac{1}{3}, \frac{1}{3}, \frac{1}{3}\right) \\
\text { AP- }\left(\frac{1}{3}, \frac{1}{3}, \frac{1}{3}\right) \\
\text { AP- }\left(\frac{1}{3}, \frac{1}{3}, \frac{1}{3}\right) \\
-\end{array}$ & $\begin{array}{l}\text { AP- }\left(\frac{1}{3}, \frac{1}{3}, \frac{1}{3}\right) \\
\text { AP- }\left(\frac{1}{3}, \frac{1}{3}, \frac{1}{3}\right) \\
\text { AP- }\left(\frac{1}{3}, \frac{1}{3}, \frac{1}{3}\right)\end{array}$ & $\begin{array}{l}\text { - } \\
\text { AP- }\left(\frac{1}{3}, \frac{1}{3}, \frac{1}{3}\right) \\
\text { AP- }\left(\frac{1}{3}, \frac{1}{3}, \frac{1}{3}\right) \\
\text { AP- }\left(\frac{1}{3}, \frac{1}{3}, \frac{1}{3}\right)\end{array}$ & $\begin{array}{l}\text { AP- }\left(\frac{1}{3}, \frac{1}{3}, \frac{1}{3}\right) \\
\text { AP- }\left(\frac{1}{3}, \frac{1}{3}, \frac{1}{3}\right) \\
\text { AP- }\left(\frac{1}{3}, \frac{1}{3}, \frac{1}{3}\right)\end{array}$ \\
\hline & 1 & $\mathrm{AC}$ & - & - & - \\
\hline & 2 & $\mathrm{AC}$ & $\mathrm{AC}$ & $\mathrm{AC}$ & $\mathrm{AC}$ \\
\hline \multirow{2}{*}{$A_{0}^{+}$} & 10 & $\mathrm{AC}$ & $\begin{array}{l}\text { AP- }(1,0,0) \text { or } \\
\text { AP- }(0,1,0) \text { or } \\
\text { AP- }(0,0,1)\end{array}$ & $\begin{array}{l}\text { AP- }(1,0,0) \text { or } \\
\text { AP- }(0,1,0) \text { or } \\
\text { AP- }(0,0,1)\end{array}$ & $\begin{array}{l}\text { AP- }(1,0,0) \text { or } \\
\text { AP- }(0,1,0) \text { or } \\
\text { AP- }(0,0,1)\end{array}$ \\
\hline & 100 & - & $\begin{array}{l}\text { AP- }(1,0,0) \text { or } \\
\text { AP- }(0,1,0) \text { or } \\
\text { AP- }(0,0,1)\end{array}$ & $\begin{array}{l}\text { AP- }(1,0,0) \text { or } \\
\text { AP- }(0,1,0) \text { or } \\
\text { AP- }(0,0,1)\end{array}$ & $\begin{array}{l}\text { AP- }(1,0,0) \text { or } \\
\text { AP- }(0,1,0) \text { or } \\
\text { AP- }(0,0,1)\end{array}$ \\
\hline \multirow{4}{*}{$A_{-1}^{+}$} & 1 & $\mathrm{AC}$ & - & - & - \\
\hline & 2 & $\mathrm{AC}$ & $\begin{array}{l}\text { AP- }(1,0,0) \text { or } \\
\text { AP- }(0,1,0) \text { or } \\
\text { AP- }(0,0,1)\end{array}$ & $\mathrm{AC}$ & $\mathrm{AC}$ \\
\hline & 10 & $\mathrm{AC}$ & $\begin{array}{l}\mathrm{AP}-(1,0,0) \text { or } \\
\mathrm{AP}-(0,1,0) \text { or } \\
\mathrm{AP}-(0,0,1)\end{array}$ & $\begin{array}{l}\mathrm{AP}-(1,0,0) \text { or } \\
\mathrm{AP}-(0,1,0) \text { or } \\
\mathrm{AP}-(0,0,1)\end{array}$ & $\begin{array}{l}\text { AP- }(1,0,0) \text { or } \\
\text { AP- }(0,1,0) \text { or } \\
\text { AP- }(0,0,1)\end{array}$ \\
\hline & 100 & - & $\begin{array}{l}\text { AP- }(1,0,0) \text { or } \\
\text { AP- }(0,1,0) \text { or } \\
\text { AP- }(0,0,1)\end{array}$ & $\begin{array}{l}\text { AP- }(1,0,0) \text { or } \\
\text { AP- }(0,1,0) \text { or } \\
\text { AP- }(0,0,1)\end{array}$ & $\begin{array}{l}\text { AP- }(1,0,0) \text { or } \\
\text { AP- }(0,1,0) \text { or } \\
\text { AP- }(0,0,1)\end{array}$ \\
\hline
\end{tabular}

Table 3.5: The frequency dynamics for 48 combinations of different matrices, models/scenarios and radii. Here we describe the frequency dynamics for a single run as the evolutionary outcome per combination. The symbol "_" means no spatial dynamics are observed as the total population becomes zero. The symbols "AP- $(1,0,0)$ ", "AP- $(0,1,0)$ " and "AP- $(0,0,1)$ " mean that the frequency dynamics are attracted to $(1,0,0),(0,1,0)$ and $(0,0,1)$, respectively. The symbol "AC" means an attracting cycle is observed.

of $A_{1}^{+}$(all scenarios included) correspond to their continuous variant with an increased radius $(=10)$, for both the averaged and single-run outcomes (see Table 3.4 and 3.5). For $A_{0}^{+}$and $A_{-1}^{+}$(which do not have ESSs), their averaged discrete-space dynamics (all scenarios included) differ strongly from their continuous ones (particularly when radius is increased to 10 and 100): The continuous dynamics are attracted to cycles, while in discrete space the random survival often occurs. Hence the discrete-space model gives a higher variance in its predictions per run.

- (Continuous-space dynamics vs. nonspatial dynamics) The stability properties of the continuous-space game averaged over 15 runs coincide with those of each single run of the corresponding case, independently of the size of radii. Therefore, here we will discuss the average outcomes. With $A_{1}^{+}$, the attracting point of the continuous-space dynamics corresponds to the ESS of the nonspatial dynamics. For the other two matrices which do not have ESSs, the discrepancy between the continuous-space model 
and the nonspatial one occurs: The continuous-space dynamics are attracted to a cycle (for both $A_{0}^{+}$and $A_{-1}^{+}$), while the nonspatial dynamics are either forming a closed orbit (for $A_{0}^{+}$, see Figure $3.2 \mathrm{~b}$ ) or are attracted to a pure-strategy attractor. (for $A_{-1}^{+}$see Figure 3.2c).

- (Discrete-space dynamics vs. nonspatial dynamics) For $A_{1}^{+}$its relation between the discrete-space and nonspatial games is similar to its relation between the continuousspace and nonspatial games. For $A_{0}^{+}$, neither the averaged nor the single-run discretespace dynamics correspond to the nonspatial ones, regardless of scenarios and radii: the single-run ones are attracted to one of the vertices (i.e., the so-called random survival occurs here), while the nonspatial dynamics are forming a closed orbit (see Figure 3.2b). For $A_{-1}^{+}$, the single-run discrete-space dynamics often do not correspond to the nonspatial ones, regardless of scenarios and radii. The matrices $A_{0}^{+}$and $A_{-1}^{+}$ do not have nonspatial ESSs, while the discrete-space games with these matrices reach spatial equilibria.

We provide the plots of frequency dynamics over 10000 generations for every $A_{\varepsilon}^{+}$given this case study in Appendix B.1 (page 149).

\subsubsection{Case study 3: Impact of the lifespan}

In case studies 3.4.4 and 3.4.5, all focal individuals (as parents) are removed from the game field after finishing their actions in the current generation. Hence all individuals have a lifespan of one generation. In this case study, we increase their lifespan to 5 generations: Individuals are not removed from the space until they reach their lifespan, and if they stay in the game field they are selected to take their actions such as density-dependent interactions, frequency-dependent actions, reproduction and dispersal. We examine the population and frequency dynamics of the 48 game (matrix, model/scenario and radius) combinations, while the lifespan of individuals is increased to 5 generations. The number of runs is kept the same as in the case study 3.4.5.

\section{Population dynamics}

At the end of the game, where we either observe saturation or extinction, the population size (averaged over 15 runs) for each combination of the 48 options is shown in Table 3.6. Here we do not report the population size for every single run as its variance among the 15 runs is very small (less than $1 \%$ of the average population size). In Table 3.6 we observe that an increased lifespan enlarges the population size particularly for the discrete-space model (crossing three scenarios) when compared to the results of Table 3.3 (where the lifespan is one generation): A longer lifespan may increase one individual's reproductive possibility, as a result more offspring are produced overall. This is to be expected as no death process is introduced to the system. 


\begin{tabular}{|c|c|c|c|c|c|c|}
\hline$\#$ & Radii & $\begin{array}{l}\text { Density } \\
\text { limit }\end{array}$ & Continuous & $\begin{array}{l}\text { Discrete } \\
\text { no } \\
\text { placement }\end{array}$ & $\begin{array}{l}\text { Discrete } \\
\text { stochastic } \\
\text { placement }\end{array}$ & $\begin{array}{l}\text { Discrete } \\
\text { deterministic } \\
\text { placement }\end{array}$ \\
\hline \multirow{4}{*}{$A_{1}^{+}$} & 1 & 5 & 15910.3 & - & 7896 & 7993.4 \\
\hline & 2 & 13 & 11674.2 & 6303.7 & 8204 & 8089.8 \\
\hline & 10 & 317 & 10493.4 & 6245.1 & 6245.1 & 6245.1 \\
\hline & 100 & 10000 & 10132.7 & 6178.8 & 8289.9 & 8294.0 \\
\hline \multirow{4}{*}{$A_{0}^{+}$} & 1 & 5 & 15632.0 & - & 8196.5 & 8203.4 \\
\hline & 2 & 13 & 11364.1 & 6184.7 & 8336.4 & 8241.3 \\
\hline & 10 & 317 & 10523.4 & 5978.5 & 8226.6 & 8177.3 \\
\hline & 100 & 10000 & 10127.8 & 6212.8 & 8220.9 & 8394.0 \\
\hline \multirow{4}{*}{$A_{-1}^{+}$} & 1 & 5 & 15503.4 & - & 8300.6 & 8188.0 \\
\hline & 2 & 13 & 11533.2 & 6400.1 & 8233.7 & 8283.7 \\
\hline & 10 & 317 & 10536.9 & 6350.5 & 8240.6 & 8279.0 \\
\hline & 100 & 10000 & 10211.0 & 6110.8 & 8351.1 & 8234.4 \\
\hline
\end{tabular}

Table 3.6: The population size at the end of the game (averaged over 15 runs), given 48 combinations of matrices, models/scenarios and radii. Here the lifespan is increased to 5 generations.

\section{Frequency dynamics}

At saturation, the observed frequency dynamics (averaged over 15 runs) are reported in Table 3.7. In Table 3.8 we report the observed frequency dynamics for single run per combination. For comparison to the nonspatial game, we refer to the replicator dynamics introduced in Section 3.4.1. Except for the case when interactions are global (radius $=100$ ), the outcomes (of both averaged and single-run) of this case study (when lifespan $=5$ ) are similar to the case study 3.4.5 (when lifespan $=1$ ). Therefore, below we talk only about our observations regarding global interaction:

- (Continuous-space dynamics vs. discrete-space dynamics) The discrete-space (including all scenarios) and continuous-space dynamics correspond to each other for $A_{1}^{+}$and $A_{-1}^{+}$, no matter whether the outcomes are averaged or not. The spatial dynamics are all attracted to $\left(\frac{1}{3}, \frac{1}{3}, \frac{1}{3}\right)$. For $A_{0}^{+}$, its continuous-space dynamics are attracted to a cycle while its discrete-space dynamics (regardless of scenarios) are attracted to $\left(\frac{1}{3}, \frac{1}{3}, \frac{1}{3}\right)$.

- (Continuous-space dynamics vs. nonspatial dynamics) The averaged continuous-space dynamics (in Table 3.7) correspond to the nonspatial ones for matrices $A_{1}^{+}$and $A_{-1}^{+}$, while the single-run continuous-space dynamics (in Table 3.8) correspond to the nonspatial dynamics only for matrices $A_{1}^{+}$. For $A_{0}^{+}$we always observe an attracting cycle as an averaged or single-run evolutionary outcome. For $A_{-1}^{+}$we observe the random survival in the continuous-space game. Please note that here the random survival also occurs in the continuous space when interactions are global: In this case, the probability to interact with a certain type equals to the frequency of this type in the population, and thus the assumption regarding interactions in the continuous-space game is equivalent to that of the nonspatial one. As a result, the continuous-space 


\begin{tabular}{|c|c|c|c|c|c|}
\hline \# & Radii & Continuous & $\begin{array}{l}\text { Discrete } \\
\text { no } \\
\text { placement }\end{array}$ & $\begin{array}{l}\text { Discrete } \\
\text { stochastic } \\
\text { placement }\end{array}$ & $\begin{array}{l}\text { Discrete } \\
\text { deterministic } \\
\text { placement }\end{array}$ \\
\hline \multirow{4}{*}{$A_{1}^{+}$} & 1 & $\mathrm{AP}-\left(\frac{1}{3}, \frac{1}{3}, \frac{1}{3}\right)$ & - & $\overline{\mathrm{AC}}$ & $\mathrm{AC}$ \\
\hline & 2 & $\mathrm{AP}-\left(\frac{1}{3}, \frac{1}{3}, \frac{1}{3}\right)$ & & $\mathrm{AC}$ & $\mathrm{AC}$ \\
\hline & 10 & $\mathrm{AP}-\left(\frac{1}{3}, \frac{1}{3}, \frac{1}{3}\right)$ & AP- $\left(\frac{1}{3}, \frac{1}{3}, \frac{1}{3}\right)$ & AP- $\left(\frac{1}{3}, \frac{1}{3}, \frac{1}{3}\right)$ & $\mathrm{AP}-\left(\frac{1}{3}, \frac{1}{3}, \frac{1}{3}\right)$ \\
\hline & 100 & $\mathrm{AP}-\left(\frac{1}{3}, \frac{1}{3}, \frac{1}{3}\right)$ & $\mathrm{AP}-\left(\frac{1}{3}, \frac{1}{3}, \frac{1}{3}\right)$ & $\mathrm{AP}-\left(\frac{1}{3}, \frac{1}{3}, \frac{1}{3}\right)$ & AP- $\left(\frac{1}{3}, \frac{1}{3}, \frac{1}{3}\right)$ \\
\hline \multirow{4}{*}{$A_{0}^{+}$} & 1 & $\mathrm{AC}$ & - & $\mathrm{AC}$ & $\mathrm{AC}$ \\
\hline & 2 & $\mathrm{AC}$ & $\mathrm{AC}$ & $\mathrm{AC}$ & $\mathrm{AC}$ \\
\hline & 10 & $\mathrm{AC}$ & RS & RS & $\mathrm{RS}$ \\
\hline & 100 & $\mathrm{AC}$ & RS & RS & $\mathrm{RS}$ \\
\hline \multirow{4}{*}{$A_{-1}^{+}$} & 1 & $\mathrm{AC}$ & - & $\mathrm{AC}$ & $\mathrm{AC}$ \\
\hline & 2 & $\mathrm{AC}$ & $\mathrm{AC}$ & $\mathrm{AC}$ & $\mathrm{AC}$ \\
\hline & 10 & $\mathrm{AC}$ & RS & RS & RS \\
\hline & 100 & RS & RS & RS & RS \\
\hline
\end{tabular}

Table 3.7: The frequency dynamics given 48 combinations of different matrices, models/scenarios and radii. Here we describe the averaged frequency dynamics (over 15 runs) as the evolutionary outcome per combination. The symbol "-" indicates the total population goes extinct. The symbol "AP- $\left(\frac{1}{3}, \frac{1}{3}, \frac{1}{3}\right)$ " indicates that the frequency dynamics are attracted to $\left(\frac{1}{3}, \frac{1}{3}, \frac{1}{3}\right)$. The symbol "AC" indicates that the frequency dynamics are attracted to a cycle. The symbol "RS" indicates random survival in at least one out of 15 runs. Here the lifespan is 5 generations.

dynamics behave similarly to the nonspatial ones, while the nonspatial dynamics of $A_{-1}^{+}$starting close to $\left(\frac{1}{3}, \frac{1}{3}, \frac{1}{3}\right)$ converge to one of the three pure-strategy attractors.

- (Discrete-space dynamics vs. nonspatial dynamics) The relation between the discretespace dynamics and the nonspatial dynamics is similar to the relation between the continuous-space dynamics and the nonspatial dynamics, except for $A_{0}^{+}$: the single-run discrete-space dynamics show that only one type stays in the game field (independently of scenarios) after 10000 generations, while the corresponding nonspatial dynamics stay at $\left(\frac{1}{3}, \frac{1}{3}, \frac{1}{3}\right)$.

The plots of frequency dynamics over 10000 generations for every $A_{\varepsilon}^{+}$can be found in Appendix B.2 (page 149). .

\subsection{Conclusion \& discussion}

In this chapter we have compared the replicator dynamics with the dynamics of discretespace and continuous-space games. We have applied agent-based modeling to simulate the spatial games and tracked the population and frequency dynamics for a class of enhanced rock-scissors-paper games.

Our conclusions can be divided into two groups: Those relating the discrete-space dynamics with the continuous-space dynamics and those comparing the nonspatial dynamics with the spatial dynamics. 


\begin{tabular}{|c|c|c|c|c|c|}
\hline$\#$ & Radii & Continuous & $\begin{array}{l}\text { Discrete } \\
\text { no } \\
\text { placement }\end{array}$ & $\begin{array}{l}\text { Discrete } \\
\text { stochastic } \\
\text { placement }\end{array}$ & $\begin{array}{l}\text { Discrete } \\
\text { deterministic } \\
\text { placement }\end{array}$ \\
\hline \multirow{4}{*}{$A_{1}^{+}$} & 1 & $\mathrm{AP}-\left(\frac{1}{3}, \frac{1}{3}, \frac{1}{3}\right)$ & - & $\mathrm{AC}$ & $\mathrm{AC}$ \\
\hline & 2 & $\mathrm{AP}-\left(\frac{1}{3}, \frac{1}{3}, \frac{1}{3}\right)$ & & & \\
\hline & 10 & $\mathrm{AP}-\left(\frac{1}{3}, \frac{1}{3}, \frac{1}{3}\right)$ & $\mathrm{AP}-\left(\frac{1}{3}, \frac{1}{3}, \frac{1}{3}\right)$ & $\mathrm{AP}-\left(\frac{1}{3}, \frac{1}{3}, \frac{1}{3}\right)$ & $\mathrm{AP}-\left(\frac{1}{3}, \frac{1}{3}, \frac{1}{3}\right)$ \\
\hline & 100 & $\mathrm{AP}-\left(\frac{1}{3}, \frac{1}{3}, \frac{1}{3}\right)$ & $\mathrm{AP}-\left(\frac{1}{3}, \frac{1}{3}, \frac{1}{3}\right)$ & $\mathrm{AP}-\left(\frac{1}{3}, \frac{1}{3}, \frac{1}{3}\right)$ & $\mathrm{AP}-\left(\frac{1}{3}, \frac{1}{3}, \frac{1}{3}\right)$ \\
\hline \multirow{7}{*}{$A_{0}^{+}$} & 1 & $\mathrm{AC}$ & - & $\mathrm{AC}$ & $\mathrm{AC}$ \\
\hline & 2 & $\mathrm{AC}$ & $\overline{\mathrm{AC}}$ & $\overline{\mathrm{AC}}$ & $\overline{\mathrm{AC}}$ \\
\hline & & & $\mathrm{AP}-(1,0,0)$ or & $\mathrm{AP}-(1,0,0)$ or & $\mathrm{AP}-(1,0,0)$ or \\
\hline & 10 & $\mathrm{AC}$ & $\mathrm{AP}-(0,1,0)$ or & $\mathrm{AP}-(0,1,0)$ or & $\mathrm{AP}-(0,1,0)$ or \\
\hline & & & $\mathrm{AP}-(0,0,1)$ & $\mathrm{AP}-(0,0,1)$ & $\mathrm{AP}-(0,0,1)$ \\
\hline & & & $\mathrm{AP}-(1,0,0)$ or & $\mathrm{AP}-(1,0,0)$ or & AP- $(1,0,0)$ or \\
\hline & 100 & $\mathrm{AC}$ & $\begin{array}{l}\mathrm{AP}-(0,1,0) \text { or } \\
\mathrm{AP}-(0,0,1)\end{array}$ & $\begin{array}{l}\mathrm{AP}-(0,1,0) \text { or } \\
\mathrm{AP}-(0,0,1)\end{array}$ & $\begin{array}{l}\text { AP- }(0,1,0) \text { or } \\
\text { AP- }(0,0,1)\end{array}$ \\
\hline \multirow{6}{*}{$A_{-1}^{+}$} & 1 & $\mathrm{AC}$ & - & $\mathrm{AC}$ & $\mathrm{AC}$ \\
\hline & 2 & $\mathrm{AC}$ & $\mathrm{AC}$ & $\mathrm{AC}$ & $\mathrm{AC}$ \\
\hline & & & $\mathrm{AP}-(1,0,0)$ or & $\mathrm{AP}-(1,0,0)$ or & $\mathrm{AP}-(1,0,0)$ or \\
\hline & 10 & $\mathrm{AC}$ & $\mathrm{AP}-(0,1,0)$ or & $\mathrm{AP}-(0,1,0)$ or & $\mathrm{AP}-(0,1,0)$ or \\
\hline & & & $\mathrm{AP}-(0,0,1)$ & $\mathrm{AP}-(0,0,1)$ & AP- $(0,0,1)$ \\
\hline & 100 & $\begin{array}{l}\text { AP- }(1,0,0) \text { or } \\
\text { AP- }(0,1,0) \text { or } \\
\text { AP- }(0,0,1)\end{array}$ & $\begin{array}{l}\text { AP- }(1,0,0) \text { or } \\
\text { AP- }(0,1,0) \text { or } \\
\text { AP- }(0,0,1)\end{array}$ & $\begin{array}{l}\text { AP- }(1,0,0) \text { or } \\
\text { AP- }(0,1,0) \text { or } \\
\text { AP- }(0,0,1)\end{array}$ & $\begin{array}{l}\text { AP- }(1,0,0) \text { or } \\
\text { AP- }(0,1,0) \text { or } \\
\text { AP- }(0,0,1)\end{array}$ \\
\hline
\end{tabular}

Table 3.8: The frequency dynamics given 48 combinations of matrices, scenarios and radii. Here we describe the frequency dynamics for a single run as the evolutionary outcome per combination. The symbol "-" means no spatial dynamics are observed as the total population becomes zero. The symbols "AP- $(1,0,0)$ ", "AP- $(0,1,0)$ " and "AP- $(0,0,1)$ " mean that the frequency dynamics are attracted to $(1,0,0),(0,1,0)$ and $(0,0,1)$, respectively. The symbol "AC" means the frequency dynamics are attracted to a cycle. Here the lifespan is increased to 5 generations.

The major difference between dynamics of the discrete-space game and the continuousspace game is their different population size at saturation: While the continuous-space game has a high population size of at least 10000 individuals, the discrete-space model allows for only about 4000 individuals with "stochastic placement" and "deterministic placement" scenarios and for about 2000 individuals with the "no placement" scenario. This difference is caused by the dispersal conflict happening when more individuals try to place their offspring into the same position in the discrete space. The dispersal conflict sometimes leads the discrete-space dynamics to extinction, especially when the interaction radius is very small.

These different ecological dynamics at saturation may lead to very different frequency dynamics, for example some of the interacting types may go extinct in a discrete space while staying around in a continuous space. Particularly, when the lifespan is short, over $50 \%$ of the case studies with the discrete-space game show the saturated population composed of only one type, while this is not observed in the continuous-space model. We observe that the random survival arising in the discrete space model causes much higher variance in the 
discrete-space dynamics when compared to the continuous-space dynamics. When lifespan is increased, the population size at saturation increases in both types of spatial games and more stable dynamics (attracting points or cycles) are observed. If the individuals stay in the game field and engage in the game for more generations, they may increase their reproductive possibilities and hence increase the survival of their types at saturation. In line with earlier studies (Kerr et al., 2002; Uyttendaele and Thuijsman, 2015), our observation for $A_{-1}^{+}$from Table 3.8 (where we report the single-run result and compare the discrete-space frequency dynamics of $A_{-1}^{+}$when radius is 2 and 100, respectively) matches the well-examined statement that local interactions promote coexistence of all three types in a RSP game.

Whether we observe discrepancies between the continuous-space model and the discretespace model, seems to depend on the nonspatial game stability properties. When the nonspatial game has an ESS (i.e., $A_{1}^{+}$), the discrete-space and continuous-space models give very similar results, and further when the interaction is global (i.e., radius is 100), the two types of spatial dynamics give similar frequency results as the nonspatial ones, if the lifespan is not too small. When the nonspatial game has no ESS (i.e., $A_{0}^{+}$and $A_{-1}^{+}$), the spatial models still converge to an attractor or an attracting cycle.

Another major difference between the discrete-space and continuous-space models is their different simulation efficiency (i.e., average time used per run). Throughout all simulations (in Sections 3.4.4, 3.4.5 and 3.4.6), we observe that the discrete-space model (including the "no placement", "stochastic placement" and "deterministic placement" scenarios) is much more efficient than that of the continuous-space one. For example, in Figure 3.10 we report the simulation time used in both spatial models, when we examine the population and frequency dynamics in Section 3.4.6 (the case study with an increased lifespan). For every model (continuous-space model, discrete-space model with "no placement" scenario, with "stochastic placement" scenario or with "deterministic placement" scenario) and every radius $(=1,2,10$ or 100$)$, we calculate the average time used per run (out of $15 \times 3$ runs $)^{17}$ for simulating dynamics up to 10000 generations. Figure 3.10 shows that the average time used per run of discrete-space model (independently of scenarios) is much lower than that of the continuous-space model, regardless of the size of the interaction radius $(=1,2,10$ and 100$)$.

So far, our spatial models do not address the issue of stability against mutations. Examining stability properties in that aspect should certainly be part of future explorations.

Future work is required if we want a more general understanding of the relation between the spatial models, as here we confined ourselves to only one class of matrix games and we did not go into very complex extensions of our models (with stochastic death process, natural disasters, etc.). However, based on our results, we consider that when the interactions are global, spatial models are not at all necessary, while if the interactions are local, a spatial model would provide a more realistic setting. However, one has to think very carefully

\footnotetext{
${ }^{17}$ Here we average all runs used for the three matrices $A_{1}^{+}, A_{0}^{+}$and $A_{-1}^{+}$. For each matrix in every model (continuous-space model, discrete-space model with "no placement" scenario, with "stochastic placement" scenario or with "deterministic placement" scenario) and every radius $(=1,2,10$ or 100$)$, there are 15 runs, hence in total there are $15 \times 3$ runs for all matrices.
} 


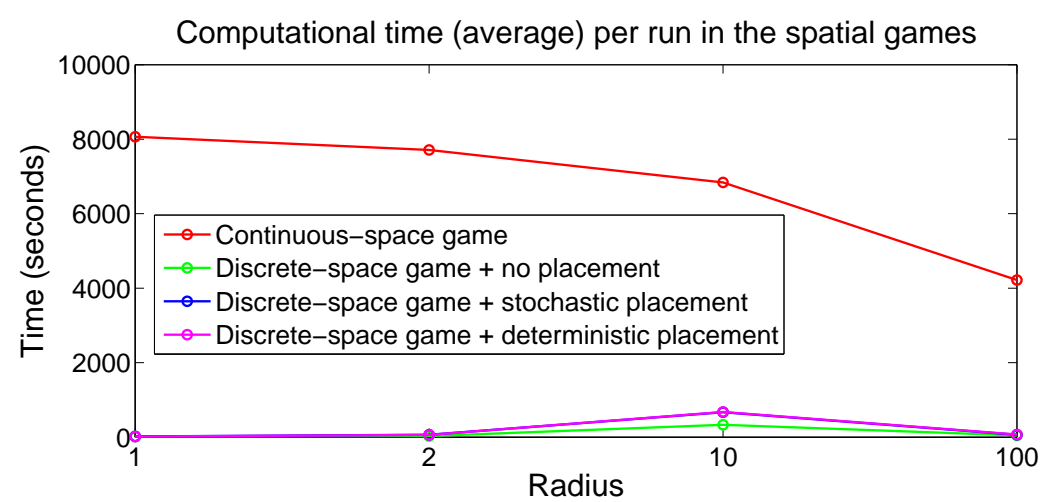

Figure 3.10: Comparing the average time (seconds) per run of discrete-space model to that of continuous-space model

whether the discrete-space or continuous-space game is a better modeling choice as their predictions generally differ. 


\section{A game-theoretical approach to microbial coexistence}

\section{Contents}

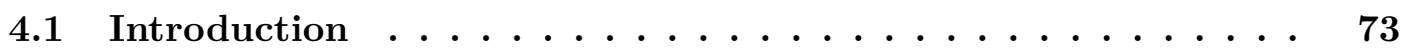

4.2 The game model ...................... 75

4.2 .1 The basic model . . . . . . . . . . . . . 76

4.2 .2 Quorum sensing model . . . . . . . . . . . . . 77

4.3 Case studies on coexistence $\ldots \ldots \ldots \ldots \ldots \ldots$

4.3.1 Case study 1: Impact of the interaction matrix density . . . . . . 79

4.3.2 Case study 2: The number of neighbors . . . . . . . . . . . 79

4.3.3 Case study 3: Basic model vs. quorum sensing model . . . . . . . 81

4.4 Conclusion \& discussion $\ldots \ldots \ldots \ldots \ldots$ 


\section{Abstract}

Huge numbers of microbes coexist in almost all habitats of our planet. Their interactions are governed by complex mechanisms, where both competition for resources and toxin production play important roles. Our goal is to understand the key mechanisms that lead to microbial coexistence. In this chapter we study several possible scenarios of microbial interactions and we analyze whether or not they can lead to coexistence of different microbial species. To achieve this, we model microbial interactions as a discrete-space game on a regular grid that mimics some of the key features of the local interactions of microbes, assuming that microbes of different species have a more or less identical morphology and are initially well mixed. Aside of the game field, the interactions of the microbes of different species are governed by the so-called interaction matrix, which defines whether or not a microbial individual of a particular species kills an individual in its neighborhood and by the so-called quorum sensing threshold, which defines how many individuals of a particular species need to be present in the neighborhood of a focal cell in order to be able to kill it. We observe that the order in which focal cells are selected to be interacted with may have a minor impact on the coexistence rate. More importantly, the coexistence rate is negatively correlated with the number of neighbors of each cell in the grid and with the density of the interaction matrix, while it is positively correlated with the quorum sensing limit.

Keywords: Discrete-space game, Local interactions, Microbial species, Bacterial strains, Quorum sensing, Agent-based models, Game theory

Adapted from: Abrudan Monica, You Li, Staňková Kateřina, Thuijsman Frank, 2016. A game theoretical approach to microbial coexistence. In Thuijsman Frank and Wagener Florian, editors, Advances in Dynamic and Evolutionary Games. Annals of the International Society of Dynamic Games, volume 14, chapter 13, pages 267-282. Springer. 


\subsection{Introduction}

Microbes outnumber by far any other living organisms on Earth (Dykhuizen, 1998). There are more microbes on Earth than stars in the universe and it is thought that $90 \%$ of the cells associated with a human body are not ours, but belong to microorganisms. With the help of new sequencing techniques, such as pyrosequencing, even environments considered sterile until a short time ago, like the uterus (Funkhouser and Bordenstein, 2013) and mother milk (Hunt et al., 2011), were found to host microbes. Our faces are not clean either: recent research found that Demodex mites might be colonizing all of us (Thoemmes et al., 2014).

What is fascinating about microbes is not just their astronomically high numbers, but also their very high species diversity. It was reported that 242 healthy individuals were found to host more than 1200 different microbes (Consortium, 2012) and a gram of soil can contain up to 8000 different operational taxonomic units (OTUs) (Delmont et al., 2011).

These amazing numbers raise an obvious question: How is the diversity maintained? Understanding the mechanisms that maintain microbial diversity could prove to be very beneficial to humans as microbial diversity plays a vital role in necessary human functions such as digestion (Ley et al., 2008): Well-balanced commensal species in a human body could prevent invasion of pathogens, keeping the human-body ecosystem healthy (Sonnenberg et al., 2012).

Studying interactions between microbes of different species is thought to be a good approach for understanding the mechanisms that drive diversity. That is why ecologists have been studying diverse microbial communities and their competition networks, networks of mutuality, and food webs (Verhoef and Morin, 2010). Topology of the interaction matrices/networks is a key indicator of the species' dynamics in a given environment (Dunne et al., 2002; Bascompte et al., 2006; Montoya et al., 2006).

Microbial interactions often take place within communities in versatile environments where microbes are exposed to each other, even when observed in Petri dishes or incubators. These observations have led us to modeling local microbial interactions as a spatial game, in an attempt to understand the processes leading to microbial coexistence. Before we define this spatial game, several concepts regarding microbes shall be introduced.

Microbial interaction matrices The so-called microbial interaction matrices, built on the basis of diverse experimental parameters (e.g. growth rates or amount of produced toxins) are built by biologists to measure microbes' ability to eliminate each other. In the laboratory, the ability of one microbial species to inhibit another one is analyzed by (i) inoculating the products (i.e., molecules) of this species into an incubator with the other species colony and (ii) recording and observing the development of the species in the incubator. A classical experiment with two species of E. coli (Chao and Levin, 1981) showed that competition is regulated by the ability of the species to produce toxins, as well as by their initial densities. Kerr et al. (2002) showed that three species of E. coli can interact in rock-scissors-paper (RSP) fashion if their growth rates as well as sensitivity to toxins are externally controlled.

Taking this line of reasoning further, we assume that interactions inside a community of 
different microbial species can be described through an $n \times n$ binary matrix $A=\left(a_{i j}\right)$ (where $n$ is the number of species and $i, j \in\{1, \ldots, n\}$ ), which describes the outcomes of pairwise interactions between individuals belonging to all species present. If entry $a_{i j}$ is 1 , then an individual of species $i$ kills species $j$, if it is 0 , it does not. Please note here that the matrix $A$ is referred to as an interaction matrix, which is different from a payoff matrix (or a fitness matrix) introduced in Chapters 1, 3 and 5.

While one can argue that in reality the entries of the interaction matrix should be expressed by the probabilities $p_{i j} \in[0,1]$ at which species $i$ can kill species $j$, it would be very difficult to derive these probabilities in laboratory measurements and, therefore, experimentalists confine themselves to discrete $0 / 1$ values. In this chapter, we will confine ourselves to this simplification as well, bearing in mind that the situation considered is a special case of the real-world situation.

Diversity of interaction matrices In our case studies, we will classify different interaction matrices according to their density, where the matrix density is the proportion of 1's among all its entries. The density of the matrix may be a good proxy of how aggressively microbes interact, for example via secretion of killing toxins observed in the bacterial world, where one bacterial strain inhibits another one. Vetsigian et al. (2011) characterized a set of 59 strains of Streptomyces and found that $43 \%$ of the interactions assessed were cases of inhibition. Kinkel et al. (2014) reported that in seven communities of Streptomyces collected from seven different geographic locations, frequencies of inhibitions between sympatric isolates varied between $10 \%$ and $33 \%$. In two different communities of Streptomyces collected from Leidse Hout in the Netherlands rates of inhibition of $33 \%$ and $43 \%$, respectively, were found. In communities of $E$. coli the inhibition is less common, with $30 \%$ of $E$. coli strains producing colicin in natural environment (Riley and Gordon, 1999). 70\% of the E. coli strains assessed were resistant to at least one colicin, while $30 \%$ were resistant to all colicin produced. In a community of 25 strains of Streptococcus pneumoniae $22 \%$ of the interactions were cases of inhibition, where all strains were capable of producing toxins and all species were vulnerable to at least one toxin. Interaction matrices corresponding to these different experiments differ in their densities, see Table 4.1. In all studies presented, the interaction matrices were constructed based on pairwise interactions: In every community, each strain was tested whether it can secrete toxins that kill the other strains.

\begin{tabular}{lll}
\hline Species & $\begin{array}{l}\text { Number of } \\
\text { strains }\end{array}$ & $\begin{array}{l}\text { Density of the interac- } \\
\text { tion matrix }\end{array}$ \\
\hline Streptomyces & 59 & 0.43 \\
\hline 7 communities of Streptomyces & 69 & $0.1-0.33$ \\
\hline Streptomyces (unpublished data) & 23 & 0.33 \\
\hline Streptomyces (unpublished data) & 20 & 0.42 \\
\hline Streptomyces (unpublished data) & 13 & 0.25 \\
\hline E. coli & & $0.07-0.21$ \\
\hline E. coli ECOR collection & 72 & 0.022
\end{tabular}


Table 4.1: Densities of different microbial interaction matrices. Results were determined experimentally.

Quorum sensing It is important to notice that bacteria are able to communicate with one another via a mechanism, where bacteria produce chemical signal molecules (that accumulate in the environment) and interpret the density of accumulated chemicals as a proxy of (bacterial) population density. Via this communication, bacteria can assess the population density of their strains and respond to this assessment by coordinating their behavior. This form of regulation in behavior as a response to (bacterial) population density is termed quorum sensing (Miller and Bassler, 2001; Waters and Bassler, 2005). Such response may be adaptation to the amount of nutrients, defense against other microorganisms which may compete for the same nutrients, and the avoidance of toxic compounds potentially dangerous for the bacteria. As a result, bacteria are able to monitor the environment including other bacteria and to alter their behavior in response to changes in the density of the strains present in a community (Williams et al., 2007). Quorum sensing is a common aspect of microbial interactions, and, therefore, in this study, we implemented a quorum sensing model (see Section 4.3.3).

The remainder of this chapter is composed as follows. In Section 4.2 the game models and their implementation are explained. In Section 4.3 we discuss three case studies using different simulation setups to investigate key factors leading to the microbial coexistence. We end the chapter with conclusions, discussions and ideas for future work (Section 4.4).

\subsection{The game model}

We assume that microbes interact with each other in a discrete-space game. It is appropriate to consider local spatial interactions as often bacteria produce toxins and can only influence individuals who are close to them. It is reasonable to confine the space to a grid, as we assume, similarly to Kerr et al. (2002), that microbes (even if they are of different species/strains/types) have more or less identical morphology.

Let $T=\{1, \ldots, n\}$ denote the set of microbial species/type labels. For our simulations, initially there are equal numbers of individuals of each type, well mixed on a fully occupied grid. We consider two different grids:

- a square grid in which each cell has 8 neighbors (Figure 4.1, left);

- a hexagonal grid in which each cell has 6 neighbors (Figure 4.1, right).

For the sake of simplicity, we assume that each type of grid from Figure 4.1 forms a torus with periodic boundary conditions.

Two different variants of the interactions will be considered. The initial model, in which only simple interactions between cells will be considered, is introduced in Section 4.2.1, while 

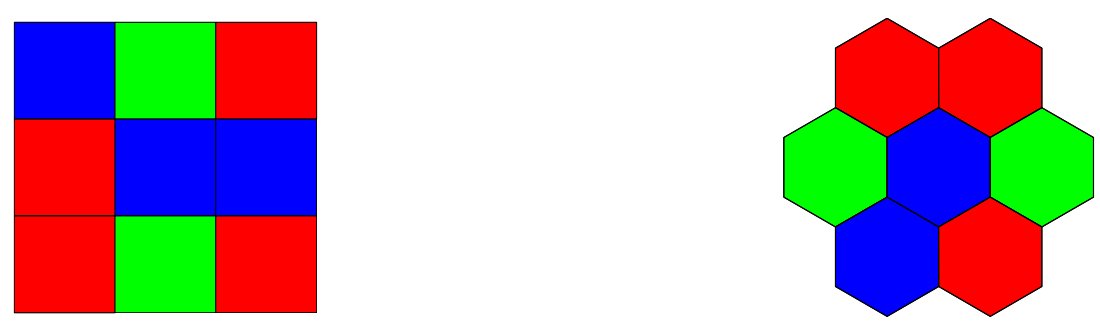

Figure 4.1: An illustration of a square grid, in which each cell has eight neighbors (left) and a hexagonal grid, in which each cell has six neighbors (right). Cells with the same color belong to the same type. In each figure only one focal cell (the central one) and its neighbors (those adjacent to the focal cell) are depicted.

a more advanced model, in which quorum sensing takes place, is introduced in Section 4.2.2. We will assume that no cells are empty, and in the remainder of this chapter, we sometimes use the term "cell" to describe an "individual" who is residing in this cell.

\subsubsection{The basic model}

We assume that the game of microbial interactions proceeds in time steps: At one time step, one cell (the so-called focal cell) and one cell from its neighborhood (where the neighborhood contains six or eight cells, depending on the grid type, see Figure 4.1) are randomly selected. If the neighbor is of the type that can kill the focal cell, it will immediately replace it by one of its own type, otherwise not. Note that the concept of time steps is different from what we call generations in Chapters $3 \& 5$, while in this chapter, one generation consists of $\eta$ time steps, where $\eta$ is the total number of cells of a grid (we confine the total number of cells of a square grid to be the same as that of a hexagonal grid). Being killed and replaced by a cell of the neighbor type represents a simple form of reproduction of that neighbor where individuals that do not kill are assumed to not reproduce. No individual can kill itself or an individual of its own type. Although suicidal behavior even on the individual level may be occasionally observed in the microbial world (e.g. the colicin producing E. coli (Engelberg-Kulka et al., 2006)), this behavior is highly rare. Moreover, we will not consider the matrices consisting of only zeros. Therefore, an interaction matrix $A=\left(a_{i j}\right)$ has the following structure:

$\quad \begin{array}{lccc}\text { type } 1 & \text { type } 2 & \ldots & \text { type } n \\ \text { type } 1 \\ \text { type } 2 \\ \vdots \\ \operatorname{type} n\end{array}\left(\begin{array}{cccc}0 & a_{12} & \ldots & a_{1 n} \\ a_{21} & 0 & \ldots & a_{2 n} \\ \vdots & \vdots & \ddots & \vdots \\ a_{n 1} & \ldots & a_{n(n-1)} & 0\end{array}\right)$,

with

$$
a_{i j}= \begin{cases}1, & \text { if type } i \text { can kill type } j \\ 0, & \text { otherwise }\end{cases}
$$


where $i \neq j$. The interactions given by matrix (4.1) can be equivalently illustrated with a directed graph with $n$ vertices, in which a directed link from $i$ to $j$ corresponds to $a_{i j}=1$, while there is no directed link from $i$ to $j$ when $a_{i j}=0$. Note that $a_{i j}=1$ does not imply that $a_{j i}=0$, for when type $i$ may be able to kill type $j$, type $j$ may also be able to kill type $i$. We examine only one matrix from each equivalence class, e.g., we will consider matrices

$$
\left(\begin{array}{lll}
0 & 0 & 0 \\
1 & 0 & 1 \\
0 & 1 & 0
\end{array}\right), \quad\left(\begin{array}{lll}
0 & 1 & 0 \\
1 & 0 & 1 \\
0 & 0 & 0
\end{array}\right), \quad\left(\begin{array}{lll}
0 & 0 & 1 \\
0 & 0 & 0 \\
1 & 1 & 0
\end{array}\right), \quad\left(\begin{array}{lll}
0 & 0 & 0 \\
0 & 0 & 1 \\
1 & 1 & 0
\end{array}\right), \quad\left(\begin{array}{lll}
0 & 1 & 1 \\
1 & 0 & 0 \\
0 & 0 & 0
\end{array}\right), \quad\left(\begin{array}{lll}
0 & 1 & 1 \\
0 & 0 & 0 \\
1 & 0 & 0
\end{array}\right),
$$

as equivalent, as they correspond to isomorphic graphs.

In the remainder of the chapter, we will refer to entire equivalence classes of matrices by their single elements.

The number of distinct interaction matrices increases rapidly with $n$ : There are 15,217 , and 9608 nontrivial equivalence classes of matrices for $n=3, n=4$, and $n=5$. respectively. These matrices can be also generated using the software Nauty (McKay and Piperno, 2014).

For a given $n$ (we consider $n=3,4$, or 5 ) and a given type of grid (hexagonal or square), simulations are always using the same initial $90 \times 90$ torus, while this initial torus is randomly generated, where cells of different strains are placed randomly in all cells of the torus with equal proportions.

\subsubsection{Quorum sensing model}

Our quorum sensing model focuses on a specific type of quorum sensing mechanism, where neighbors can kill a focal cell via their toxin secretion, if their density is above the certain threshold, called the quorum sensing threshold. Such a quorum sensing mechanism is encoded in the blp locus in Streptococcus pneumoniae (De Saizieu et al., 2000) and other microbes. The quorum sensing model extends the basic model introduced in Section 4.2.1. In this new model, the interactions follow three steps, with a prespecified quorum sensing threshold $\phi$ :

1. Selection of a focal cell (say of type $j$ ).

2. Random selection of a neighbor (say of type $i$ ) of the focal cell.

3. If $a_{i j}=1$, then the focal cell (of type $j$ ) is killed by the neighbor cell (of type $i$ ) and replaced by another cell (of type $i$ ), provided that there are at least $\phi$ cells of type $i$ in the neighborhood of the focal cell. Otherwise, the focal cell (of type $j$ ) stays alive.

In the following, we will show an example of this quorum sensing model.

Example 4.2.1. Consider a system of microbes of three types, with the interaction matrix

$$
\left(\begin{array}{lll}
0 & 0 & 0 \\
1 & 0 & 1 \\
0 & 1 & 0
\end{array}\right)
$$


and the quorum sensing threshold $\phi$ set to 2 .

Imagine a neighborhood with focal cell of type 2, as depicted in Figure 4.2a. If a neighbor of type 1 or 2 is selected, the focal cells would not be replaced as $a_{12}=a_{22}=0$. However, if a neighbor of type 3 is selected and there are at least $\phi=2$ type 3 neighbors of the focal cell, then the focal cell is replaced by a cell of type 3 , as $a_{32}=1$. Such a replacement is illustrated in Figure 4.2b.

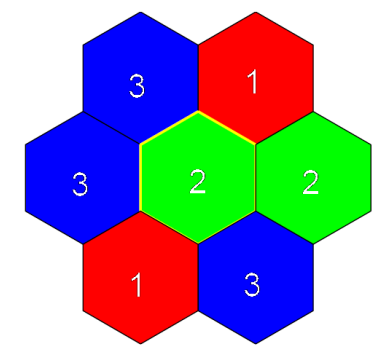

(a) A focal cell of type 2 and its neighbors

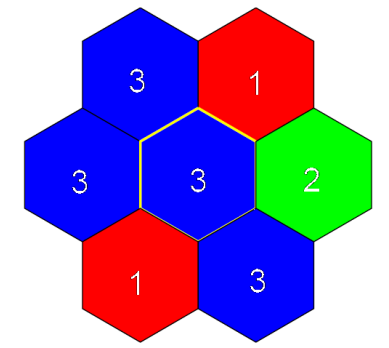

(b) Changing the focal cell type when a neighbor of type 3 was selected

Figure 4.2: An illustration of the quorum sensing model

\subsection{Case studies on coexistence}

In this section we examine several conditions which may influence coexistence of cells of different types. By coexistence we mean that each type which was initially in the field is still represented by at least one cell at the end of the simulation. For each equivalence class of interaction matrices of sizes $3 \times 3$ and $4 \times 4$, respectively, we ran 100 simulations $(15 \times 100$ and $217 \times 100$ simulations with $3 \times 3$ and $4 \times 4$ matrices in total, respectively) starting from the same, but randomly generated, initial field. For each interaction matrix of size $5 \times 5$ we ran 10 simulations (thus $9608 \times 10$ simulations in total). We only ran 10 simulations in the latter case because of its high computational time for the $90 \times 90$ torus. Our main focus is the coexistence rate, defined as the portion of all runs per matrix where coexistence is observed.

As an interaction matrix defines which cell types are able to kill other types, and as the density of an interaction matrix can be seen as a measure of how likely cells will kill each other, in the case studies we will cluster our results with respect to interaction matrices of the same densities. For example, there are in total four $3 \times 3$ matrices with density $\frac{4}{9}$ :

$$
\left(\begin{array}{lll}
0 & 1 & 0 \\
1 & 0 & 0 \\
1 & 1 & 0
\end{array}\right), \quad\left(\begin{array}{lll}
0 & 1 & 1 \\
1 & 0 & 0 \\
1 & 0 & 0
\end{array}\right), \quad\left(\begin{array}{lll}
0 & 0 & 1 \\
1 & 0 & 0 \\
1 & 1 & 0
\end{array}\right), \quad\left(\begin{array}{lll}
0 & 1 & 1 \\
0 & 0 & 0 \\
1 & 1 & 0
\end{array}\right) .
$$

and those matrices will therefore belong to the same group of interaction matrices, identified by the same matrix density $\frac{4}{9}$. 
For a given $n(=3,4$ and 5$)$, we then analyze the coexistence rate per (group with the same) density, i.e. the proportion of runs in this group in which coexistence occurs.

Next we examine the dependence of the coexistence rate on the order of selecting focal cells, interaction matrix density, and quorum sensing threshold.

\subsubsection{Case study 1: Impact of the interaction matrix density}

This case study analyzes the coexistence properties of the model introduced in Section 4.2.1, in relation to the interaction matrix density. Case studies with three, four, and five types will be carried out. For this case study, the game torus consists of 8100 square cells and one generation therefore involves 8100 time steps. Moreover, two different ways of how the focal cells are selected in each generation are considered in the case studies:

- Partially random: In each generation all 8100 cells are selected as focal cells, one by one, in a random order. The basic interactions introduced in Section 4.2.1 are performed for each of them and the field is updated immediately after each time step (i.e., each interaction takes place for one selected focal cell). The total number of generations is set to 12000 .

- Random: We randomly select 8100 cells as focal cells and at each time step we perform the basic interactions introduced in Section 4.2.1. As in the previous case, the field is updated immediately per time step. We call these simulations random, because after each interaction a new focal cell is selected randomly from all the field. Therefore, some cells may be selected as focal cells more than once, while some may not be updated at all.

In Figure 4.3 we report the coexistence rates obtained in the simulations with both partially randomly and randomly selected focal cells. Figures $4.3 \mathrm{a}-4.3 \mathrm{c}$ show the results when $n=3,4$ and 5, respectively. In Figure 4.3d we show the results including all $3 \times 3$, $4 \times 4$ and $5 \times 5$ interaction matrices. We observe that, independently of the manner how focal cells are selected (i.e., partially random or random), a higher matrix density leads to less coexistence, and this observation holds when $n=3,4,5$ or when we look across all $3 \times 3$, $4 \times 4$ and $5 \times 5$ matrices. This is to be expected as a cell is more likely to be killed by its neighbors with an interaction matrix with higher density. Moreover, the coexistence rates decrease with the matrix density, independently of a manner how focal cells are selected.

\subsubsection{Case study 2: The number of neighbors}

In this case study we experiment again with the basic model described in Section 4.2.1. We examine how the coexistence rate differs for two types of grids: one where each cell has six neighbors and one where each cell has eight neighbors, as introduced in Section 4.2.1. As before, interactions with three, four, and five types are studied. For each interaction matrix of size $3 \times 3$ and $4 \times 4$, respectively, we ran 100 simulations, while for each interaction matrix 


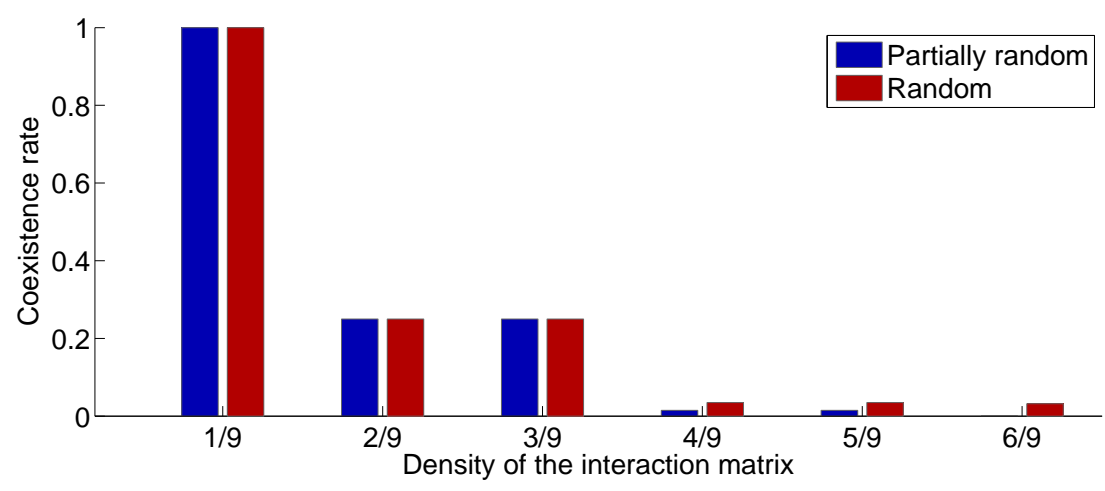

(a)

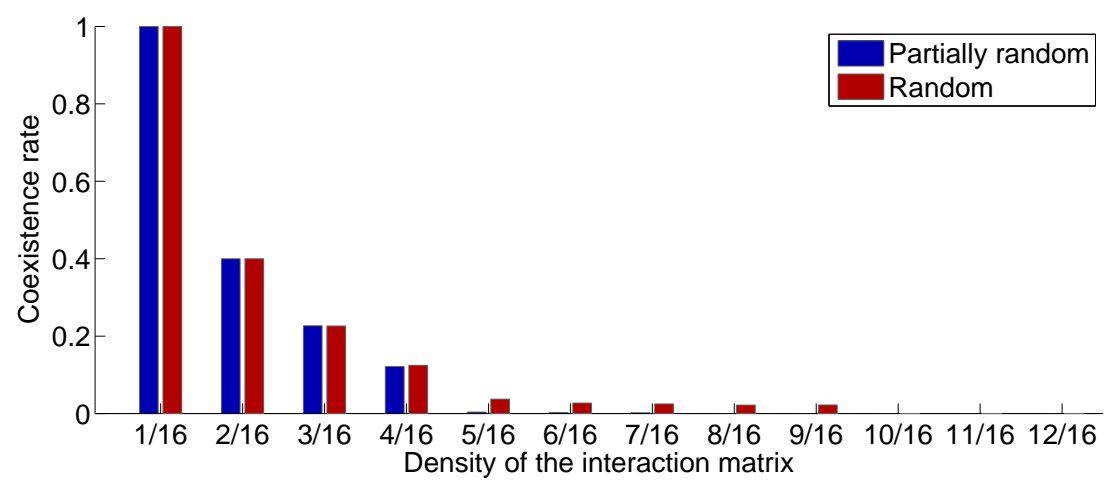

(b)

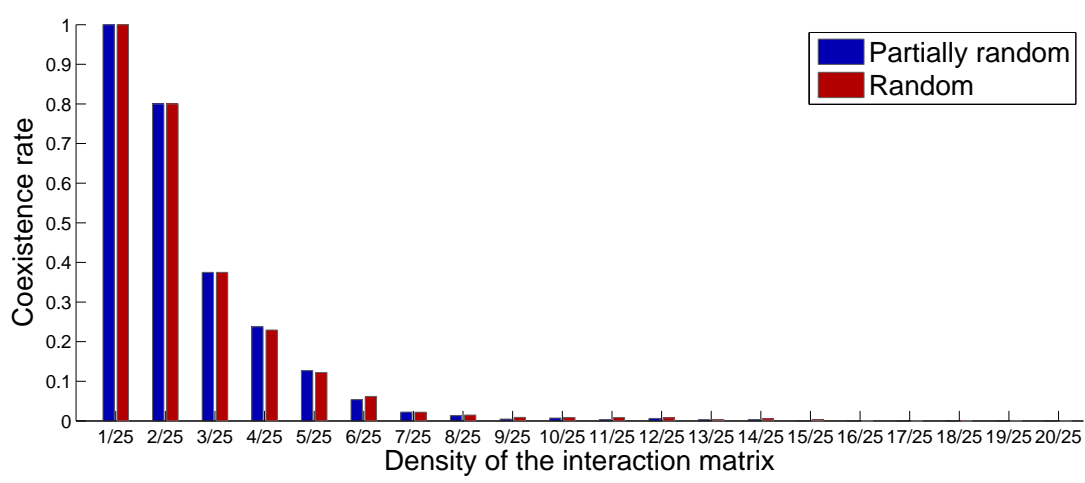

(c)

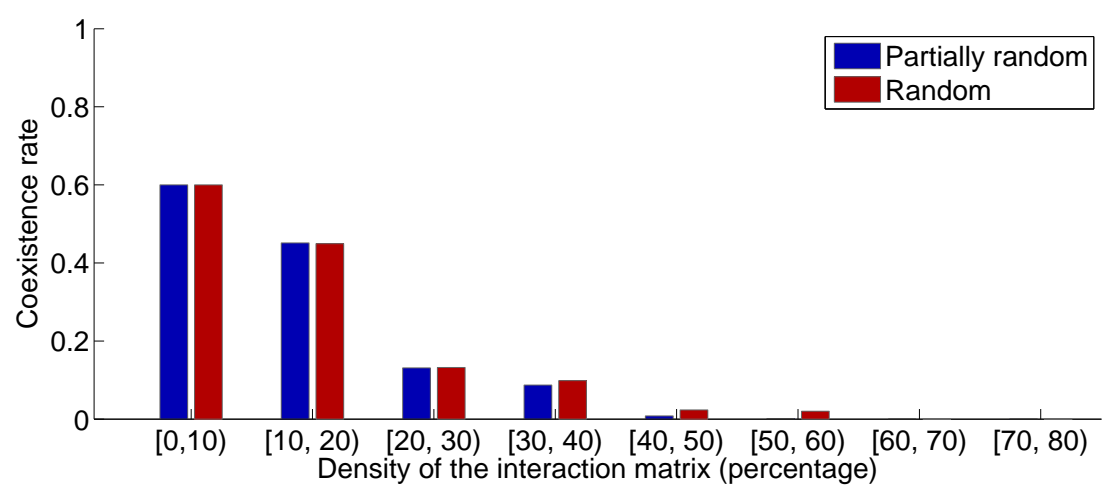

(d)

Figure 4.3: Coexistence rates for different densities of interaction matrices, for the two manners of selecting focal cells. Results for (a) $3 \times 3$ matrices, (b) $4 \times 4$ matrices, (c) $5 \times 5$ matrices and (d) all matrices. 
of size $5 \times 5$ we ran 10 simulations. The initial conditions are as before. We assume that focal cells are selected randomly.

Results are summarized in Figure 4.4. We observe that, independently of the neighborhood size, a higher matrix density leads to less coexistence, and this observation holds when $n=3,4,5$ and therefore also when we look across all $3 \times 3,4 \times 4$ and $5 \times 5$ matrices. Moreover, Figure 4.4 indicates that, independently of the density (of the matrices), the coexistence rate, achieved for the hexagonal grid, is larger than or at least equal to that achieved for the square grid. The results (Figure 4.4) confirm that increase of the neighborhood size decreases the coexistence rate. Intuitively, this seems natural, as more neighbors increase chances that a focal cell meets its predator and is killed.

\subsubsection{Case study 3: Basic model vs. quorum sensing model}

We compare the simulation outcomes of the basic model (described in Section 4.2.1) with the simulation outcomes of the quorum sensing model (described in Section 4.2.2).

Both models were ran 100 times for all interaction matrices of size $3 \times 3$ and $4 \times 4$, respectively, and 10 times for interaction matrices of size $5 \times 5$, on a hexagonal grid and with a random selection of focal cells. The initial conditions are the same as before.

In the quorum sensing model we considered the situations where the quorum sensing threshold $\phi$ is set to 1, 2, 3 and 4, respectively. Figure 4.5 shows our results.

Note that as $\phi$ increases, each neighborhood has to have more cells of the same type in order to be able to kill its focal cell, which is harder to come across. Therefore, increasing $\phi$ increases the coexistence rate. When the quorum sensing threshold is very high (4 in our case) the matrix density does not visibly influence coexistence, probably because with such a high quorum sensing threshold focal cells are rarely killed for any interaction matrix and all types coexist, as can be seen in Figure 4.5.

\subsection{Conclusion \& discussion}

In this chapter we investigated how microbial coexistence depends on various assumptions regarding microbial interactions, using simple models. We observed that increasing the quorum sensing threshold, decreasing the interaction matrix density, and reducing the neighborhood size all lead to increase of the coexistence rate, while we also observed that the order of selecting focal cells for interactions with others impact the microbial coexistence as well, but to a much lower magnitude.

The interaction matrices used in our simulations play a very different role from the fitness matrices frequently used in evolutionary game theory. While the fitness matrices specify the reproductive success of the interacting individuals, the interaction matrices define whether or not a focal individual may be killed and replaced by one of its neighbors. Therefore, the interactions in our models are not those of the replicator dynamics. However, as the mechanism of interaction, i.e., being killed and replaced by a cell of the neighbor type, simulates one particular way of reproduction of that neighbor, it is interesting to see how 


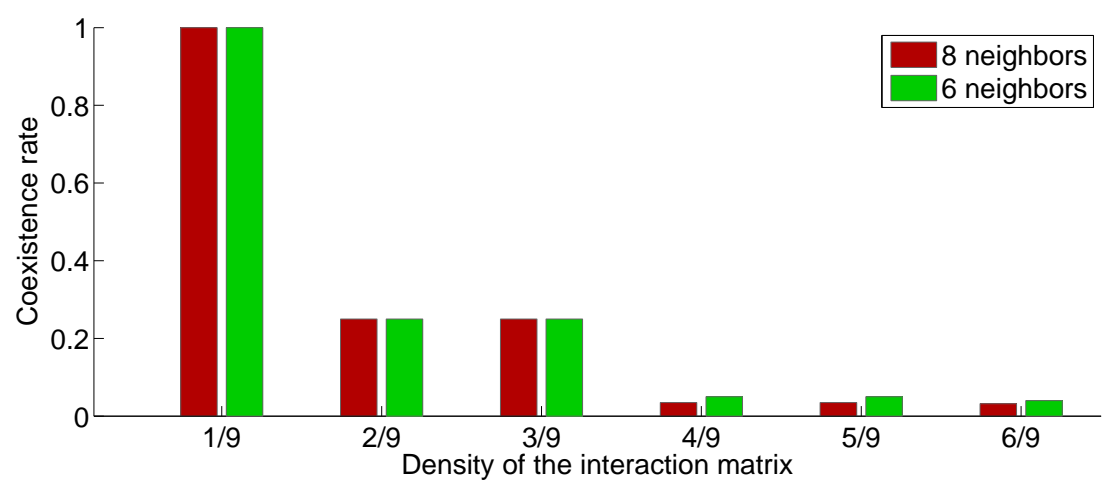

(a)

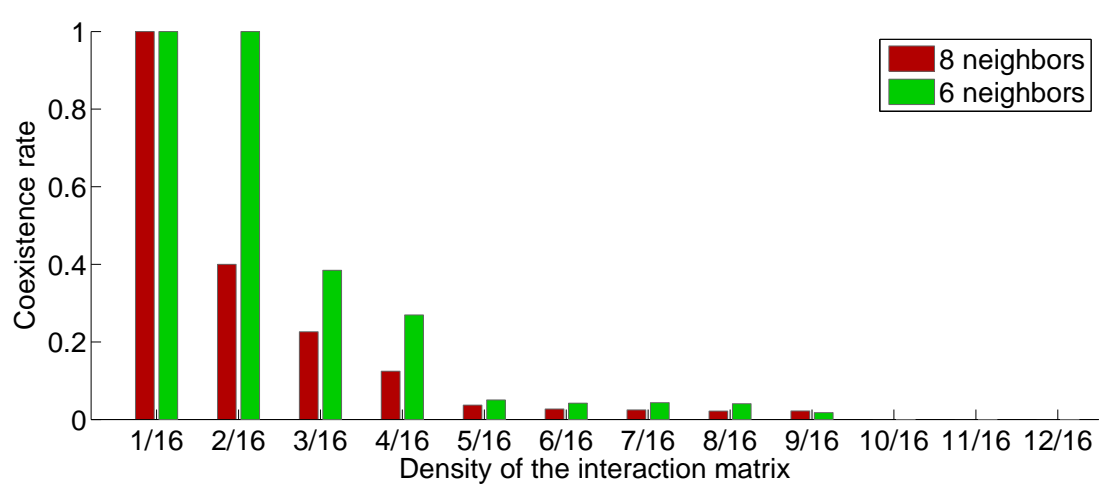

(b)

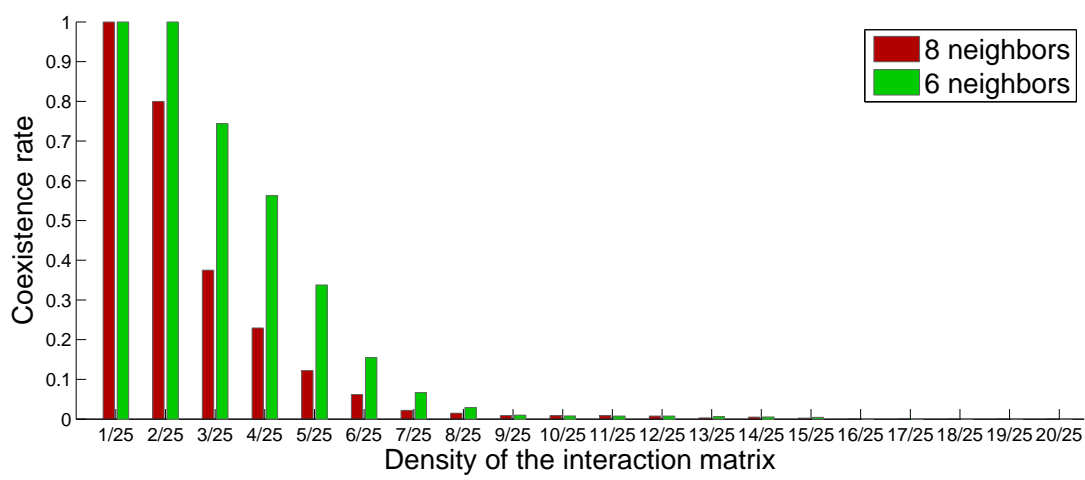

(c)

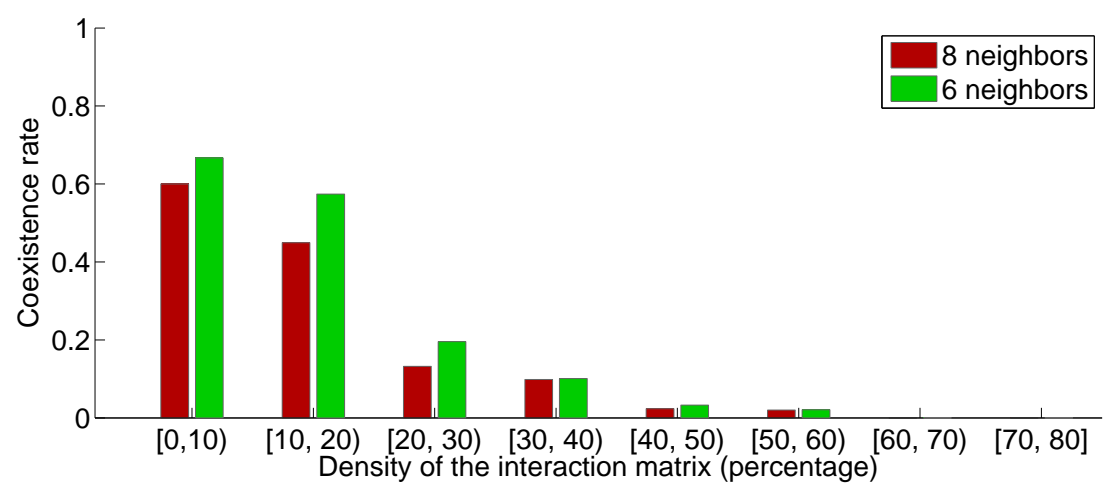

(d)

Figure 4.4: Coexistence rates for different matrix densities, obtained under two sets of simulations with a square grid (neighborhood size is 8) and a hexagonal grid (neighborhood size is 6). Results of (a) $3 \times 3$ matrices, (b) $4 \times 4$ matrices, (c) $5 \times 5$ matrices and (d) all matrices. 


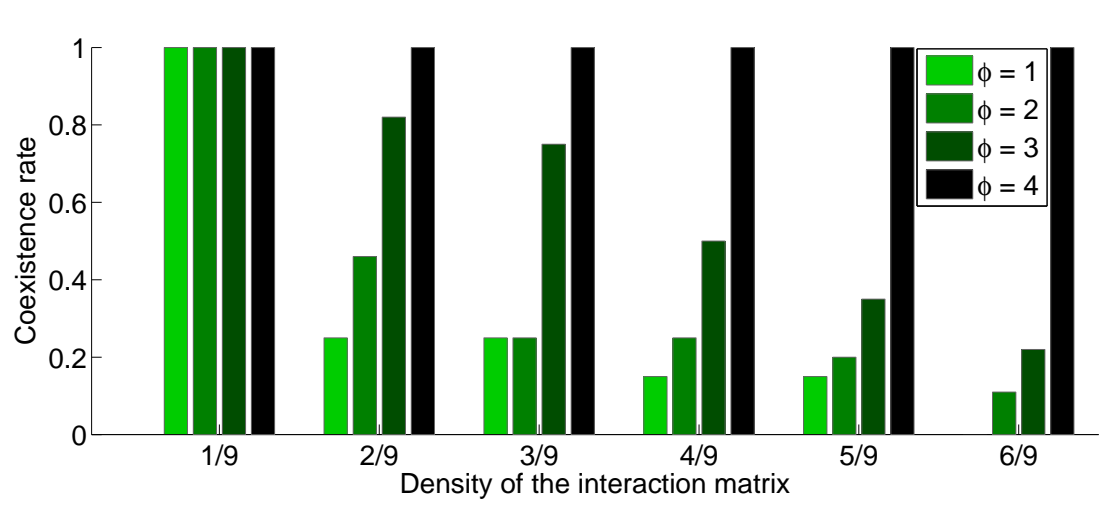

(a)

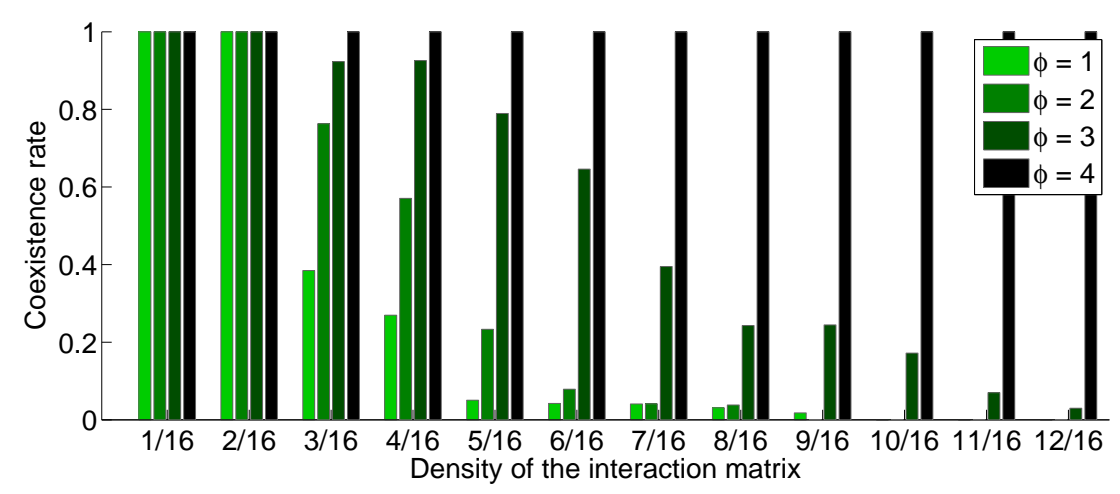

(b)

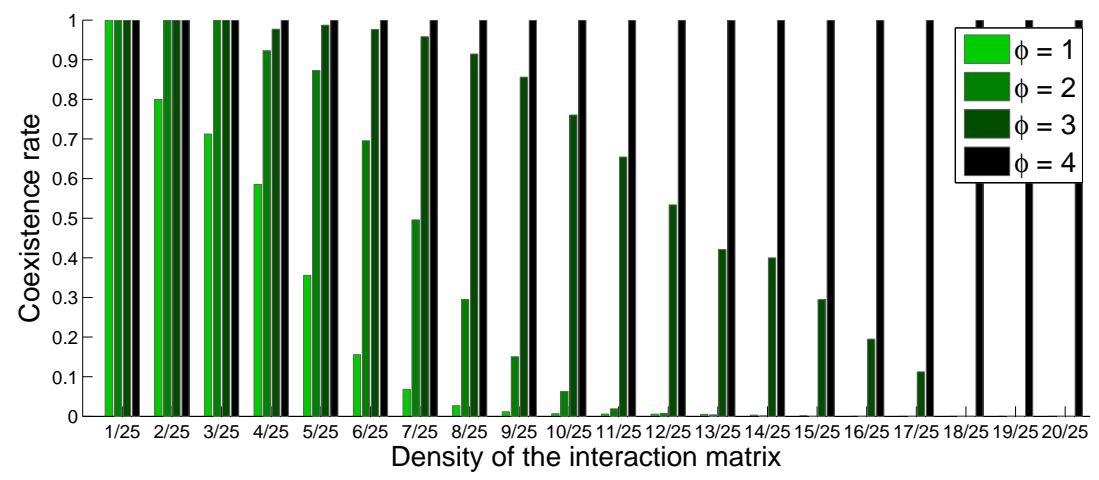

(c)

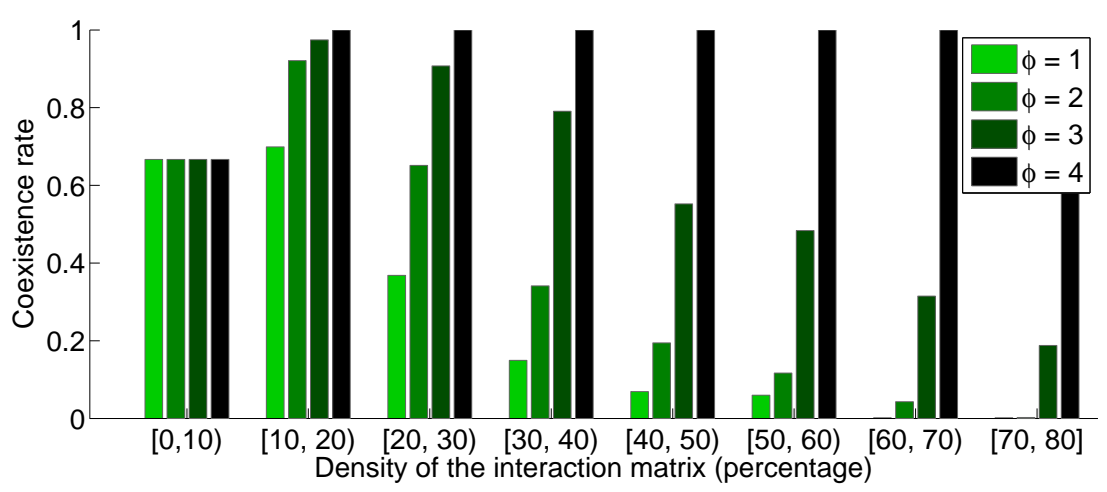

(d)

Figure 4.5: Coexistence rate with respect to different matrix densities and $\phi$. Results of (a) $3 \times 3$ matrices, (b) $4 \times 4$ matrices, (c) $5 \times 5$ matrices and (d) all matrices, respectively, are shown. 
interaction matrices and fitness matrices relate to each other, and whether the same results can be achieved using replicator dynamics. Such a study has not been carried out yet and is a natural step for our future research.

In our simulations we observed something that one could call "coexistence by small numbers". This means that quite frequently we observed that a specific species, having less than 10 cells in our $90 \times 90$ torus, would not go extinct simply because of being completely surrounded and protected by neighbors which cannot kill it. Consider for example the following interaction matrix:

$$
\left(\begin{array}{lll}
0 & 0 & 0 \\
1 & 0 & 0 \\
0 & 0 & 0
\end{array}\right)
$$

The first species is killed by the second species (position $(2,1)$ in the matrix), but it is immune to the third one (position $(3,1)$ in the matrix). Few individuals of the first species can survive 12000 generations, by hiding inside islands formed by the third strain. Figure 4.6 shows a snapshot of the grid at the last generation (from one of the 100 simulations). This

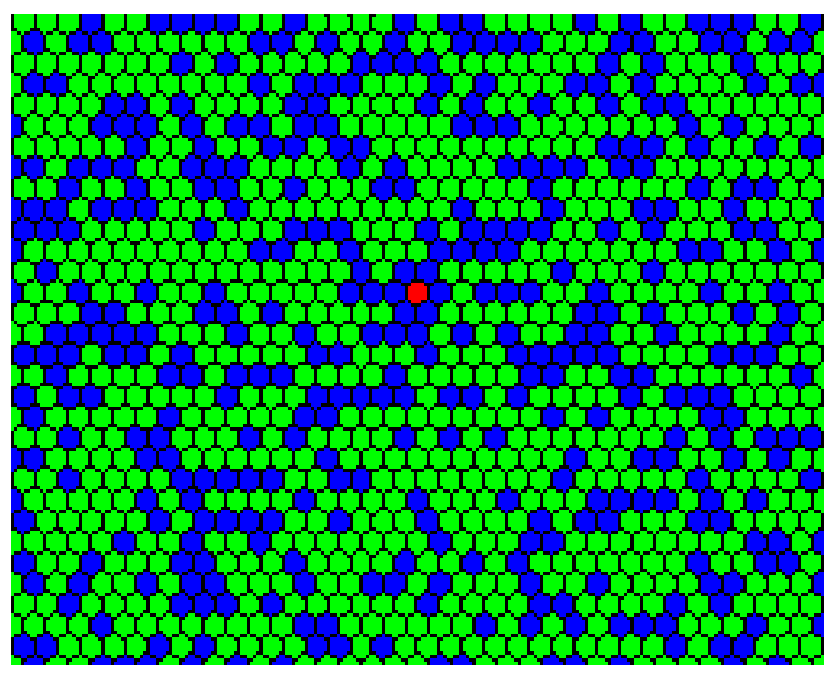

Figure 4.6: An example of coexistence by small numbers. This is a snapshot of field at the last generation of a simulation where strains follow the rules described in the interaction matrix (4.5).

result is consistent with experimental studies where it was found that few strains dominate one habitat (McGill et al., 2007) and many others are present in low numbers. While Ren et al. (2015) demonstrated that the presence of the rare species is vital for the equilibrium of the microbial community, more work is needed to understand how this relates to "coexistence by small numbers". Moreover, this coexistence by small numbers may be a mere artifact of the tight and uniform neighborhood structures we examined, and therefore we would like to examine models with more diverse neighborhood structures, such as those in a continuous space.

Our approach has several limitations, which will be addressed in our future research. Firstly, the models presented assume that the interactions of $n$ microbial species can be reduced to a binary matrix of size $n \times n$. Although this may seem very simplistic, the design 
of the model was motivated by previous experimental studies that characterized communities of coexisting microbes in a similar fashion (the results of those experimental studies are summarized in Table 4.1). Nevertheless, with the advance of experimental techniques, we expect that future experimental characterizations of microbial communities will become more comprehensive and thus will allow us to improve on our models, by including new parameters, e.g. the probability at which one type can kill another type. Secondly, the study investigated the potential coexistence of three, four and five different microbial species. While this is informative, a future study should discuss coexistence of a higher number of strains. Thirdly, our models assume that all individual population members remain at fixed positions in space, while in reality the individuals move. We therefore wish to explore the effects of different mobility rules on coexistence and relate our models to other studies on this aspect (Reichenbach et al., 2007). Finally, in this work we defined coexistence as presence of all types initially introduced to the game, while it may be very useful to focus also on the so-called partial coexistence, where some of the types coexist and others do not. 


\section{Spatial and nonspatial games of} tumorigenesis

\section{Contents}

5.1 Introduction . . . . . . . . . . . . . . . 89

5.2 Models: Replicator dynamics and its spatial variant . . . . 91

5.2.1 Background: Metastatic castrate-resistant prostate cancer . . . . . 91

5.2 .2 Model basics . . . . . . . . . . . . . . . . . . . . . 92

5.2.3 Replicator dynamics in metastatic castrate-resistant prostate cancer 92

5.2.4 Spatial replicator dynamics in metastatic castrate-resistant prostate cancer . . . . . . . . . . . . . . . . . . 94

5.3 Tumor growth and composition . . . . . . . . . . 96

5.3.1 The effects of the mortality regime on tumor growth . . . . . . . 98

5.3.2 Spatial vs. nonspatial dynamics . . . . . . . . . . . . . . 102

5.4 Effects of the frequency-dependence radius, the dispersal radius and the density-dependence radius on spatial equilibria $\quad 105$

5.4.1 Effect of the frequency-dependence radius . . . . . . . . . . . . 105

5.4 .2 Effect of the dispersal radius . . . . . . . . . . . 106

5.4.3 Effect of the density-dependence radius . . . . . . . . . 106

5.5 Conclusion \& discussion $\ldots \ldots \ldots \ldots$. . . . . . . 108 


\section{Abstract}

Metastatic prostate cancer is initially treated with androgen deprivation therapy (ADT). However, resistance typically develops in about 1 year - a clinical condition termed metastatic castrate-resistant prostate cancer $(\mathrm{mCRPC})$. We develop and investigate a spatial game (agent-based continuous space) of mCRPC that considers three distinct cancer cell types: (i) those dependent on exogenous testosterone $\left(T^{+}\right)$, (ii) those with increased CYP17A expression that produce testosterone and provide it to the environment as a public good $\left(T^{P}\right)$, and (ii) those independent of testosterone $\left(T^{-}\right)$. The interactions within and between cancer cell types can be represented by a $3 \times 3$ matrix. Based on the known biology of this cancer there are 22 potential matrices that give roughly three major outcomes depending upon the absence (good prognosis), near absence or high frequency (poor prognosis) of $T^{-}$ cells at the evolutionarily stable strategy (ESS). When just two cell types coexist the spatial game faithfully reproduces the ESS of the corresponding matrix game. With three cell types divergences occur, in some cases just two strategies coexist in the spatial game even as a nonspatial matrix game supports all three. Discrepancies between the spatial game and nonspatial ESS happen because different cell types become more or less clumped in the spatial game - leading to non-random assortative interactions between cell types. Three key spatial scales influence the distribution and abundance of cell types in the spatial game: (i) Increasing the radius at which cells interact with each other, can lead to higher clumping of each type, (ii) Increasing the radius at which cells experience limits to population growth, can cause densely packed tumor clusters in space, (iii) Increasing the dispersal radius of daughter cells, promotes increased mixing of cell types. To our knowledge the effects of these spatial scales on eco-evolutionary dynamics have not been explored in cancer models. The fact that cancer interactions are spatially explicit and that our spatial game of mCRPC provides in general different outcomes than the nonspatial game might suggest that nonspatial models are insufficient for capturing key elements of tumorigenesis.

Keywords: Evolutionary game theory, Spatial game, Nonspatial game, Prostate cancer

Adapted from: You Li, Brown Joel. S., Thuijsman Frank, Cunningham Jessica. J., Gatenby Robert. A., Zhang Jingsong., Staňková Kateřina, (2017). Spatial vs. non-spatial eco-evolutionary dynamics in a tumor growth model. Journal of Theoretical Biology, 435: 78-97. This work is sponsored by the European Union's Horizon 2020 research and innovation program under the Marie Skłodowska-Curie grant agreement No 690817, National Institute of Health grants U54CA143970-1 and RO1CA170595, and a grant from the James S. McDonnell Foundation. 


\subsection{Introduction}

In cancer biology, tumors are viewed as complex ecosystems consisting of cancer cells, normal cells, blood vasculature, inter-cellular spaces, and various nutrients such as oxygen and glucose (Gatenby et al., 2014; Merlo et al., 2006; Orlando et al., 2012). Within this ecosystem, cancer cells, often of distinct types, compete for space and nutrients, and engage in direct interactions. Cancer cells both contribute towards and are affected by their neighborhoods (known as microenvironments) within which they consume available resources, survive and proliferate (Egeblad et al., 2010). Within these neighborhoods there are eco-evolutionary feedbacks where limiting resources influence the total abundance of cancer cells and interactions between tumor cells influence the frequency of cell types. While often modeled nonspatially, several features of tumors invite spatially-explicit models. For instance, biopsies, histological samples and MRI imaging all provide spatial information on tumor characteristics (Sottoriva et al., 2013; Waclaw et al., 2015). Pathologists often measure and score spatial distributions of cancer cell types, vasculature, immune cells, and other tumor properties (Zhang et al., 2014; Patel et al., 2014). Finally, increasingly cancer biologists recognize the ubiquity of spatial heterogeneity within tumors (Swanton, 2012; Bedard et al., 2013). This heterogeneity likely has significance for tumor progression, metastases and patient outcome (Marusyk et al., 2012).

Mathematical models of cancer have been employed to understand tumor initiation, progression and metastases (Altrock et al., 2015). Such models can be used to fit existing data, evaluate key factors relevant to cancer progression, or provide qualitative and quantitative predictions that can be experimentally validated (Altrock and Traulsen, 2009; Werner et al., 2011). Nonspatial models of cancer can be deterministic or stochastic. They can take the form of ordinary differential equations that track the dynamics of the cancer cells (often seen as growing logistically or according to a Gompertz equation (Kozusko and Bajzer, 2003)) and perhaps that of normal cells and/or immune cells. Spatially explicit models may take the form of diffusion processes framed as partial differential equations (Tomasetti et al., 2013), or the models may be agent based (Macklin and Edgerton, 2010). As agent-based models, the cancer cells or other features of the tumor may be represented on vertices of a lattice or network. Or, individual cells may occupy a space on a spatial grid described as squares or hexagons (Thalhauser et al., 2010; Perfahl et al., 2011). Finally, agent-based models can consider continuous space where the cancer cells are represented by continuously varying spatial coordinates in one, two or three dimensions (Gallaher and Anderson, 2013).

Mathematical models can consider the eco-evolutionary dynamics that occur in tumors. Here we define a cancer cell "type" as cells that share the same heritable phenotype relevant to the cancer under study. Ecological dynamics represent changes in the population size or density of cancer cells. The evolutionary dynamics consider how the heritable traits of cancer cell lineages change with time, or how the frequencies of different cancer cell types change with time. When a cancer cell's survival or proliferation probabilities are influenced by its type and the types of other cancer cells, the dynamics are frequency-dependent and therefore can be described using game theory. Evolutionary game theory (EGT) provides 
an excellent modeling tool for considering complex tumors that include several interacting cancer cell types.

EGT deals with interactions between players (Tomlinson, 1997; Beerenwinkel et al., 2015). As a game, cancer cells represent the players, their types or heritable phenotypes represent the different strategies, and survival and proliferation rates represent the payoffs. A cell's payoff will be influenced by its strategy and the strategies of others. EGT includes tools for modeling population dynamics and the evolutionary dynamics of changes in the frequency of different cancer cell types. EGT can be used to find eco-evolutionary equilibria and to evaluate their stability. When there are a finite number of different possible strategies among the cancer cells, then the evolutionary dynamics can be modeled using replicator dynamics (RD) (Hofbauer and Sigmund, 1998). RD are frequency dynamics of nonspatial evolutionary games and apply when an individual interacts with the population via "playing the field" (meaning an individual engages in a global interaction among all individuals of this population). Recent research has focused on extending RD into spatially explicit scenarios (Nowak, 2006; Ohtsuki et al., 2006; Uyttendaele and Thuijsman, 2015). Both the evolutionary dynamics and subsequent equilibria may change when space is made explicit (Hauert and Doebeli, 2004; Kerr et al., 2002; Ohtsuki and Nowak, 2006; Számadó et al., 2008; Uyttendaele et al., 2012).

Currently both RDs (Dingli et al., 2009; Basanta et al., 2012; Mateo et al., 2014) and their spatial variants (e.g., being spatially defined as occurring on a fixed lattice of a graph (Basanta et al., 2008)) are being applied to cancer. Here we use a spatially explicit agent-based approach to model cancer as an evolutionary game. First, we describe metastatic castrateresistant prostate cancer that provides the motivation for our modeling work. The biology of this cancer suggests three important cancer cell types (strategies) whose interactions and payoffs can be described with a $3 \times 3$ matrix game. We can analyze this matrix game as a nonspatial model using RD, and we develop a spatial version of the prostate cancer model where the space is continuous. Second, we find the evolutionarily stable strategies (ESS) for the nonspatial game, and the stable equilibria that arise in the spatial variant of the model. Finally, using the spatial model, we explore three relevant processes and scale dependencies that must occur in actual tumors. To our knowledge these processes and their interactions have not been collectively explored in cancer models.

The first of these processes relates to the density-dependence radius. A given tumor cell will be negatively affected by the density of other cancer cells within some neighborhood that represents the local depletion of resources or buildup of toxins. The second process relates to the frequency-dependence radius, that describes the distance at which cancer cells play the game. Up to what distance does the strategy of neighbors matter in terms of influencing the payoff to an individual tumor cell? The third process relates to the dispersal radius. Cancer cells exhibit motility and the resulting movement of cells determines the distance between two daughter cells. Prior models have not made the distinction between the three scale dependent processes of density-dependence, frequency-dependence, and dispersal. By considering continuous space, our model lends itself to examining the effects of these three scale-dependent processes on the eco-evolutionary equilibrium and on the dispersion of cancer 
cells and strategies in space.

In what follows we introduce the metastatic castrate-resistant prostate cancer models used in this paper (Section 5.2); we compare the eco-evolutionary outcomes of the nonspatial and spatial models (Section 5.3); we examine the effects of varying the three scale-dependent processes on spatial equilibria (Sections 5.4); and we discuss our results and highlight some directions for future research (Section 5.5).

\subsection{Models: Replicator dynamics and its spatial vari- ant}

\subsubsection{Background: Metastatic castrate-resistant prostate cancer}

Prostate cancer most commonly metastasizes to the bone, and several to a dozen or more secondary tumors can arise across bones of a single patient (Basanta et al., 2012; Fizazi et al., 2013). Despite no longer residing within the primary tumor of the prostate (in fact for many of these patients the prostate may have been removed), these cancer cells still retain androgen receptors and rely on testosterone produced by normal cells of the patient and distributed through the blood (Hamilton et al., 2016). We shall refer to this cancer cell type as having strategy $T^{+}$. To target these cancer cells' need for testosterone, androgen deprivation therapy (ADT) stops normal production of testosterone in the patient - a treatment termed "chemical castration" (Yao et al., 2008; Hussain et al., 2013; Tsai et al., 2013). Virtually all patients initially respond to ADT. But, metastatic prostate cancer remains an almost uniformly fatal disease because the cancer cells are able to evolve resistance. In ADT, resistance typically results in a few months or years and the disease progresses to metastatic castrate-resistant prostate cancer (mCRPC). Resistance to ADT typically occurs through two different strategies. One of these involves the upregulation of CYP17A whereby the cancer cell produces its own testosterone (Cai et al., 2011; Mostaghel et al., 2011). These we shall refer to as $T^{P}$ cells. They retain androgen receptors. The $T^{P}$ cells have the side effect of providing testosterone to their external environment where it becomes available to $T^{+}$cells. Finally, resistance to androgen deprivation can take the form of cancer cells becoming wholly independent of testosterone: $T^{-}$. Treatment of mCRPC depends on the dominant resistance strategy. When $T^{P}$ cells are that largest intratumoral population, abiraterone, a CYP17A inhibitor, is typically effective. However, even in patients who respond to abiraterone, the disease progresses usually within 1-2 years.

Here we investigate the eco-evolutionary dynamics of mCRPC by modeling the composition and dispersion patterns of the three cell types $\left(T^{+}, T^{P}\right.$ and $\left.T^{-}\right)$within a tumor. The resulting eco-evolutionary equilibria become important in that a tumor with primarily $T^{+}$ and $T^{P}$ cells can be effectively treated with drugs such as abiraterone that target androgen receptors (Ryan et al., 2013). However, tumors with high frequencies of $T^{-}$cells will be unresponsive to abiraterone and require chemotherapy. 


\subsubsection{Model basics}

Let $T=\left\{T^{+}, T^{P}, T^{-}\right\}$be the set of cell types from Section 5.2.1. Let $x_{i}, i \in T$, denote the frequency of the cells of type $i \in T$ in the population. We assume that the cancer cells interact with each other as a game. When a focal cell of type $i \in T$ interacts with a cell of type $j \in T$, the outcome is the probability that the focal cell divides and creates an offspring of type $i$. These division probabilities for interaction between all types form a payoff (fitness) matrix $A$ depicted in Table 5.1. Please consult Appendix C.1 (page 153) for details about

\begin{tabular}{l|lll} 
& $T^{+}$ & $T^{P}$ & $T^{-}$ \\
\hline$T^{+}$ & 0 & $a$ & $b$ \\
$T^{P}$ & $c$ & 0 & $d$ \\
$T^{-}$ & $e$ & $f$ & 0
\end{tabular}

Table 5.1: The fitness matrix for $T^{+}, T^{P}$ and $T^{-}$cell populations. The diagonal values have been set to 0 .

the nonspatial model corresponding to this payoff matrix.

\subsubsection{Replicator dynamics in metastatic castrate-resistant pro- state cancer}

For each type $i \in T$, the replicator dynamics (Hofbauer and Sigmund, 1998) define the time change $\dot{x}_{i}$ of its cell frequency $x_{i}$ :

$$
\dot{x}_{i}=x_{i}\left(e_{i} A x^{\top}-x A x^{\top}\right), \quad i \in T
$$

where $x=\left(x_{T^{+}}, x_{T^{P}}, x_{T^{-}}\right)$, and $e_{i}$ is the $i$-th row of a $3 \times 3$ identity matrix. Even with the same initial conditions $\left(x(0)=x_{0}\right)$, the frequency dynamics (5.1) will vary with the payoff matrix $A$. For the 22 cases, we can map the frequency trajectories and the evolutionarily stable strategies (ESSs) on a simplex (Figure 5.1). When starting from positive initial frequencies, i.e., $x(0)=\left(\frac{1}{3}, \frac{1}{3}, \frac{1}{3}\right)$, each case of our model results in a single ESS, which is the attractor for the dynamics given by (5.1). In the notation of Bomze (1983), the games we consider fall into the following two groups: (i) "no fixed point in the interior simplex" and "no edge pointwise fixed"; and (ii) they will exhibit "one fixed point in interior simplex" and "three non-corner fixed points on edges".

Based on the frequency of the $T^{-}$cells at the ESS, we can divide the 22 cases for replicator dynamics into three different groups (Table 5.2):

I. (positive) The ESS frequency of $T^{-}$cells is 0 - such tumors should respond strongly to abiraterone

II. (neutral) The ESS frequency of $T^{-}$cells is between 0 and 0.15 - such tumors may respond to abiraterone

III. (negative) The ESS frequency of $T^{-}$cells is greater than 0.15 - such tumors may correspond to non-responders 
Group I

\#1

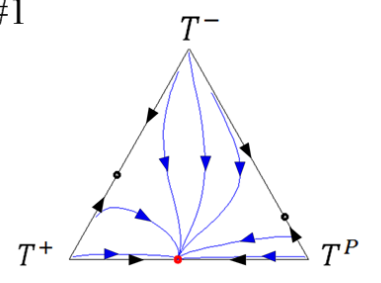

\#5

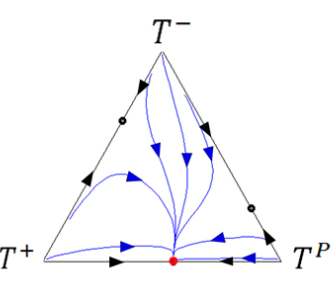

\#11

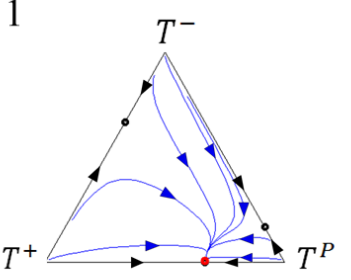

\#2

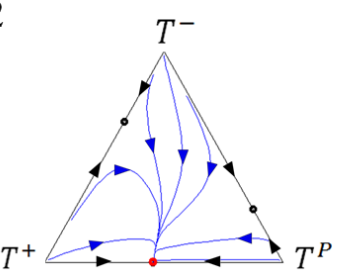

\#6

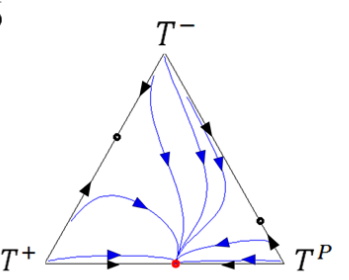

\#14

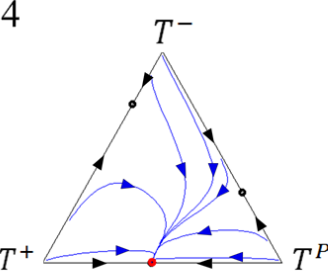

\#3

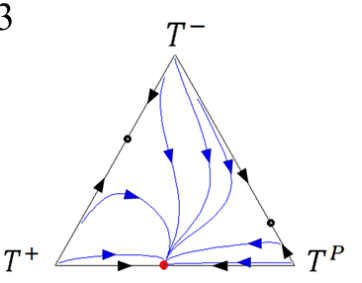

\#7

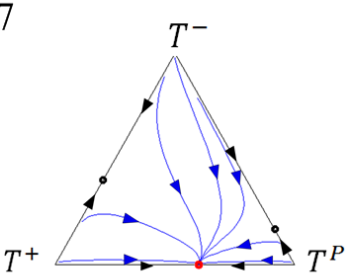

\#17

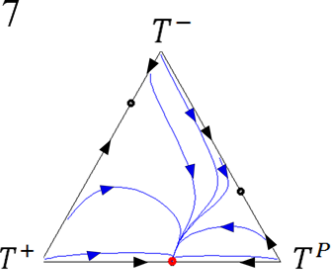

\#20
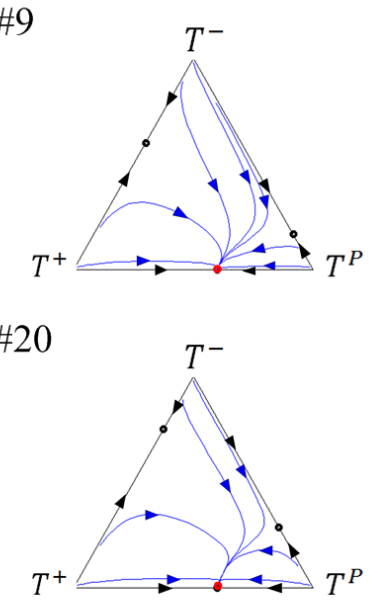

Group II

\#8

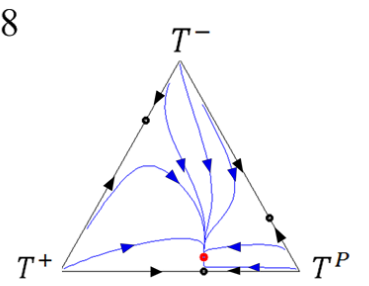

\#10

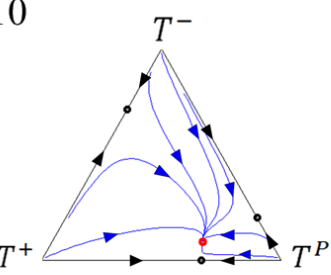

\#12

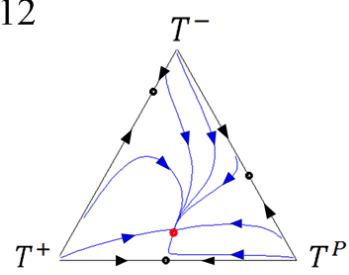

\#4

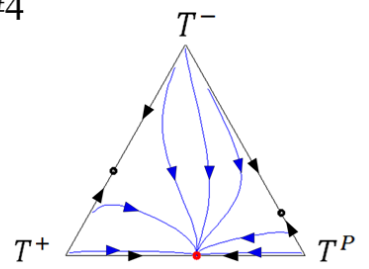

$\# 9$

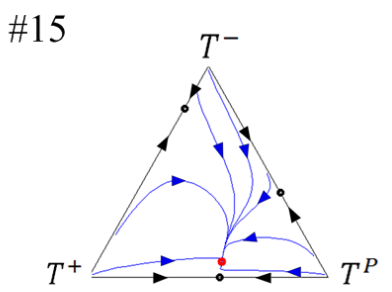

Group III

\#13

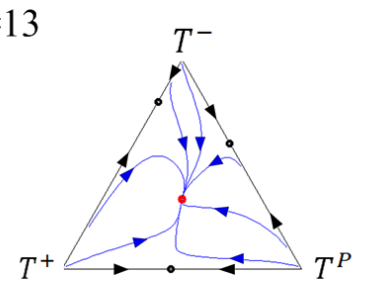

\#21

\#16

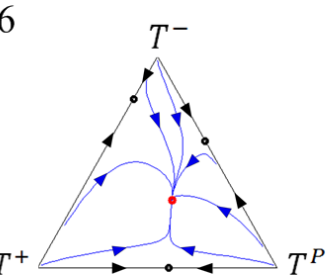

\#18

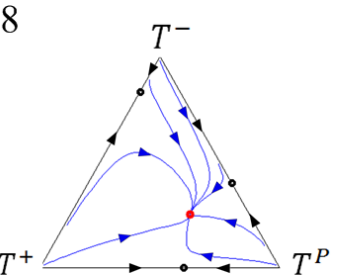

\#19

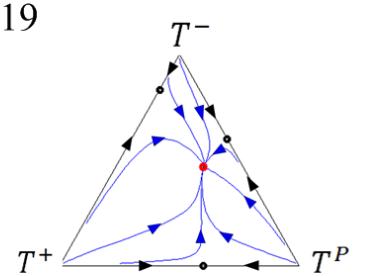

\#22
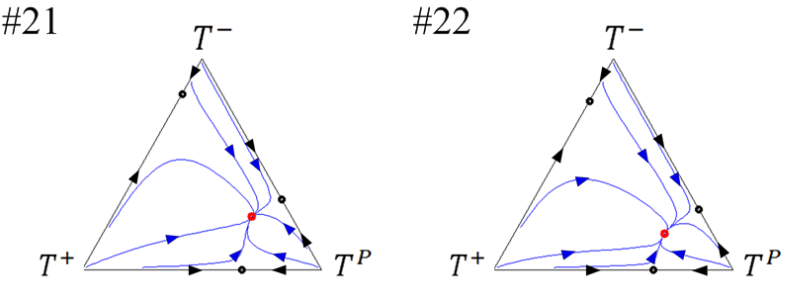

Figure 5.1: The trajectories of cell type frequencies for each matrix from Table 5.2, ordered by group. The vertices of the simplex correspond to a frequency of 1 for the corresponding cell type. The red (full) circles represent the ESSs and the black (full) circles represent the saddle points. 


\begin{tabular}{|c|c|c|c|}
\hline$\#$ & $(a, b, c, d, e, f)$ & ESS & Group \\
\hline 1 & $(0.6,0.3,0.5,0.4,0.2,0.1)$ & $\left(\frac{6}{11}, \frac{5}{11}, 0\right)$ & \multirow{12}{*}{ I (positive) } \\
\hline 2 & $(0.6,0.2,0.5,0.3,0.4,0.1)$ & $\left(\frac{6}{11}, \frac{5}{11}, 0\right)$ & \\
\hline 3 & $(0.6,0.2,0.5,0.4,0.3,0.1)$ & $\left(\frac{6}{11}, \frac{5}{11}, 0\right)$ & \\
\hline 4 & $(0.5,0.3,0.6,0.4,0.2,0.1)$ & $\left(\frac{5}{11}, \frac{6}{11}, 0\right)$ & \\
\hline 5 & $(0.5,0.2,0.6,0.3,0.4,0.1)$ & $\left(\frac{5}{11}, \frac{6}{11}, 0\right)$ & \\
\hline 6 & $(0.5,0.2,0.6,0.4,0.3,0.1)$ & $\left(\frac{5}{11}, \frac{6}{11}, 0\right)$ & \\
\hline 7 & $(0.4,0.3,0.6,0.5,0.2,0.1)$ & $\left(\frac{2}{5}, \frac{3}{5}, 0\right)$ & \\
\hline 9 & $(0.4,0.2,0.6,0.5,0.3,0.1)$ & $\left(\frac{2}{5}, \frac{3}{5}, 0\right)$ & \\
\hline 11 & $(0.3,0.2,0.6,0.5,0.4,0.1)$ & $\left(\frac{1}{3}, \frac{2}{3}, 0\right)$ & \\
\hline 14 & $(0.6,0.1,0.5,0.4,0.3,0.2)$ & $\left(\frac{6}{11}, \frac{5}{11}, 0\right)$ & \\
\hline 17 & $(0.5,0.1,0.6,0.4,0.3,0.2)$ & $\left(\frac{5}{11}, \frac{6}{11}, 0\right)$ & \\
\hline 20 & $(0.4,0.1,0.6,0.5,0.3,0.2)$ & $\left(\frac{2}{5}, \frac{3}{5}, 0\right)$ & \\
\hline 8 & $(0.4,0.2,0.6,0.3,0.5,0.1)$ & $\left(\frac{11}{30}, \frac{17}{30}, \frac{2}{30}\right)$ & \multirow{4}{*}{ II (neutral) } \\
\hline 10 & $(0.3,0.2,0.6,0.4,0.5,0.1)$ & $\left(\frac{10}{35}, \frac{22}{35}, \frac{3}{35}\right)$ & \\
\hline 12 & $(0.6,0.1,0.5,0.3,0.4,0.2)$ & $\left(\frac{14}{31}, \frac{13}{31}, \frac{4}{31}\right)$ & \\
\hline 15 & $(0.5,0.1,0.6,0.3,0.4,0.2)$ & $\left(\frac{11}{27}, \frac{14}{27}, \frac{2}{27}\right)$ & \\
\hline 13 & $(0.6,0.1,0.5,0.2,0.4,0.3)$ & $\left(\frac{1}{3}, \frac{1}{3}, \frac{1}{3}\right)$ & \multirow{6}{*}{ III (negative) } \\
\hline 16 & $(0.5,0.1,0.6,0.2,0.4,0.3)$ & $\left(\frac{7}{25}, \frac{10}{25}, \frac{8}{25}\right)$ & \\
\hline 18 & $(0.4,0.1,0.6,0.3,0.5,0.2)$ & $\left(\frac{1}{4}, \frac{2}{4}, \frac{1}{4}\right)$ & \\
\hline 19 & $(0.4,0.1,0.6,0.2,0.5,0.3)$ & $\left(\frac{5}{30}, \frac{11}{30}, \frac{14}{30}\right)$ & \\
\hline 21 & $(0.3,0.1,0.6,0.4,0.5,0.2)$ & $\left(\frac{2}{12}, \frac{7}{12}, \frac{3}{12}\right)$ & \\
\hline 22 & $(0.3,0.1,0.6,0.5,0.4,0.2)$ & $\left(\frac{7}{35}, \frac{22}{35}, \frac{6}{35}\right)$ & \\
\hline
\end{tabular}

Table 5.2: Division of the 22 cases for replicator dynamics (5.1) into 3 groups according to the ESS frequencies of $T^{-}$cells. No $T^{-}$cells (group I) indicates a highly treatable tumor, a low frequency of $T^{-}$cells indicates a moderately treatable tumor (group II), while a high frequency of $T^{-}$cells (group III) indicates an untreatable tumor (the non-responder).

\subsubsection{Spatial replicator dynamics in metastatic castrate-resistant prostate cancer}

In this section, we model the population and frequency dynamics of the prostate cancer cells as a spatial game on a continuous space. We think continuous-space models are appropriate for modeling tumor progression because the cancer cells (i) may disperse into any direction from their original positions and (ii) may have a heterogeneous and time-varying morphology even within the same tumor. Moreover, we aim to seek for ecological and evolutionary 
dynamics at both the transient and saturated phases (as introduced in Chapter 3) in a tumor. In the sense the cancer cells shall reproduce and place their daughter cells in the free space, so that the tumor grows to its saturation. It is realistic to consider a spatial model that allows a high saturated population for the tumor and, as discussed in Chapter 3, the continuousspace models handle saturation much better than the discrete-space ones. In the remainder of this chapter, whenever we use the terms "spatial game" and "spatial dynamics", what we mean are the "continuous-space game" and "continuous-space dynamics", respectively.

We imagine the cancer cells as players on a continuous torus $\Theta_{\mathbb{R}}=[0,50) \times[0,50)$ with periodic boundary conditions. Rather than having a fixed grid or lattice, cells can exist at any point on this surface. Initially, there are 99 cancer cells (33 of each type), scattered randomly within the central zone $C_{\mathbb{R}}=[20,30] \times[20,30]$ of the flat torus $\Theta_{\mathbb{R}} \cdot{ }^{18}$

For the spatial game we specify rules regarding cell death, density-dependent interactions, frequency-dependent interactions and cell proliferation, which occur in generations. During a generation all living cells are selected in a random order to undergo the following actions:

Cell death We imagine that cell death can be either stochastic or deterministic. A cancer cell dies with either a fixed probability (stochastic) or after a fixed number of generations (deterministic). If the focal cell dies, it does not undergo any further actions. Following death, the cell either stays in the field permanently or is removed after a prespecified number of generations. The combinations of deterministic or stochastic cell death and the rate at which dead cells stay in the field (5 generations, 30 generations, or permanently) generate six possible mortality regimes. (see Table 5.3). If the focal cell remains alive, it undergoes

\begin{tabular}{c||l|l}
\multirow{2}{*}{ Scenario } & \multicolumn{2}{c}{ Mortality regime } \\
\cline { 2 - 3 } & Cell death & Dead cells staying in field \\
\hline 1 & Stochastic (probability 5\%) & Short (5 generations) \\
2 & Deterministic (20 generations) & Permanently \\
3 & Stochastic (probability 5\%) & Permanently \\
4 & Deterministic (20 generations) & Long (30 generations) \\
5 & Deterministic (20 generations) & Short (5 generations) \\
6 & Stochastic (probability 5\%) & Long (30 generations) \\
\hline
\end{tabular}

Table 5.3: Six scenarios corresponding to six mortality regimes

the next action.

Density-dependent cell interactions We imagine that available space and resources are necessary for successful cell proliferation. We define the density-dependence neighborhood as a disc around the focal cell with a prespecified radius, called the density-dependence radius (Figure 5.2a). If the number of cells (itself and dead cells included) within this densitydependence neighborhood is greater than or equal to 10 cells per unit area, then the focal

\footnotetext{
${ }^{18}$ While for modeling tumorigenesis in real patients it may be more realistic to start simulations with other initial proportions of $T^{+}, T P$ and $T^{-}$, for the purpose of this research the initial condition $\left(\frac{1}{3}, \frac{1}{3}, \frac{1}{3}\right)$ seems appropriate. Moreover, in local neighborhoods the proportions will differ anyway.
} 
cell cannot proliferate and it does not move onto additional actions in the current generation. If the density of cells within the density-dependence neighborhood is less than 10 cells per unit area then the focal cell moves onto the next action.

Frequency-dependent cell interactions We imagine the cells also have a frequencydependence neighborhood, which is a disc around the focal cell with a prespecified radius, called the frequency-dependence radius (Figure 5.2b). The focal cell randomly selects a neighbor cell from the living cells occurring within this frequency-dependence neighborhood. Having selected the focal cell's "opponent" for the game we move to the last action.

Cell proliferation The probability of the focal cell undergoing cell division and producing a daughter cell is determined by the payoff from the matrix $A$ if we let the type of the focal cell be the row strategy and the type of the opponent be the column strategy (Table 5.1). If the focal cell reproduces, it generates a daughter cell of its own type. This daughter cell is not placed in the field immediately, but only after all living cells have completed their actions for the current generation. A given daughter cell is placed at a random location in the focal cell's dispersal neighborhood (Figure 5.2c). The dispersal neighborhood is a disc around the focal cell with prespecified radius, called the dispersal radius.

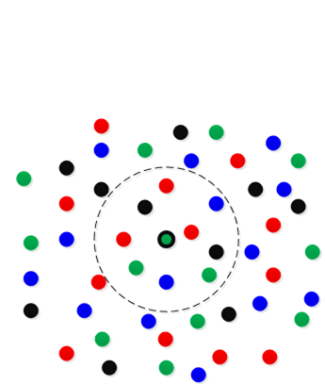

(a)

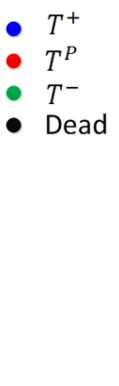

Dead

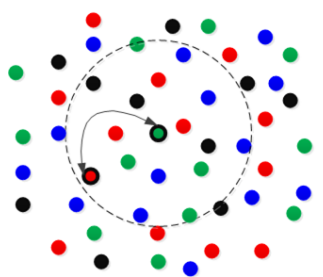

(b)

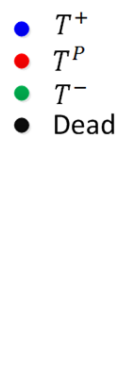

$T^{P}$
$T^{-}$
Dead

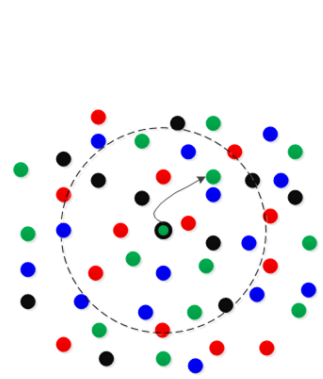

(c)

Figure 5.2: A focal cell and its three neighborhoods. (a) Density-dependence neighborhood. A focal cell might proliferate if the density of cells within the disc defined by the densitydependence radius is below a threshold. (b) Frequency-dependence neighborhood. The focal cell's likelihood of proliferating will be determined from an interaction between it and a randomly selected cell from within the disc defined by the frequency-dependence radius. (c) Dispersal neighborhood. If a focal cell proliferates, its daughter cell is placed randomly within the disc defined by the dispersal radius.

\subsection{Tumor growth and composition}

In this section we simulate tumor growth under the six different mortality regimes (Table 5.3). We compare the tumor composition of the six scenarios with the ESS of the nonspatial models for the 22 payoff matrices. In the simulations we track changes in

1. the total number of cancer cells (population dynamics) 
2. the frequencies of cell types (frequency dynamics)

3. the dispersion pattern of each cell type

Each simulation begins with 99 cells (33 per cell type), placed randomly in the central $10 \times 10$ zone of the flat torus $\Theta_{\mathbb{R}}=[0,50) \times[0,50)$. We ran simulations for 2000 generations For each combination of the mortality regime (Table 5.3) and payoff matrix, we repeated the simulation five times, in order to check whether or not the standard deviation between the five simulations is high. If the standard deviation is low, the spatial dynamics of this combination may be represented by a single run. There are 660 simulations. For these simulations, we set the density-dependence, frequency-dependence and dispersal radii to one. The density-dependent threshold for reproduction was set to 10 cells per unit area within the focal cell's density-dependence neighborhood. Without cell death, the maximum population size is 25000 as the torus has size $50 \times 50$ and 10 is the density-dependence limit per unit area.

To evaluate the spatial dynamics and for comparisons to the ESS of the nonspatial model, we need stability concepts for spatial equilibria in terms of the population densities and frequencies of the three cell types. Here, we will consider a spatial equilibrium stable if the frequencies in each two consecutive generations have a difference less than 0.001 in the 50 past generations, as stated formally in Definitions 5.3.1 and 5.3.2:

Definition 5.3.1 (Stable frequency in the spatial game). Frequency $x_{i}$ of type $i \in T$ is stable in generation $\tau$ if $\left|x_{i}(t+1)-x_{i}(t)\right| \leq 0.001$ for all generations $t \in\{\tau-49, \tau-48, \ldots, \tau\}$.

Definition 5.3.2 (Stable spatial equilibrium). A spatial equilibrium is stable in generation $\tau$ if the frequencies of all three types $\left(T^{+}, T^{P}, T^{-}\right)$are stable in this generation.

In the remainder of the paper, whenever we refer to "equilibria", what we mean is "stable equilibria", just to simplify the notation.

We might be able to observe two different types of (stable) equilibria: The transient (stable) equilibrium and saturated (stable) equilibrium. The former might occur when the total number of tumor cells is still growing, while the latter occurs once the space is saturated, i.e., once the total population of living cells as well as populations per type in the tumor do not change (due to the finite size of field $\Theta_{\mathbb{R}}$ ). In order to quantify when such equilibria occur, we define the transient and saturated equilibria in Definitions 5.3.3 and 5.3.4.

Definition 5.3.3 (Transient stable spatial equilibrium). A stable spatial equilibrium is called transient in generation $\tau$ if the sum of populations of $T^{+}, T^{P}$, and $T^{-}$cells keeps growing in generations $\{\tau-49, \tau-48, \ldots, \tau\}$.

In the following definition, we use term maximal total population, which refers to the largest total population of cells reached over the run of the simulation, including both dead and living cells to this count.

Definition 5.3.4 (Saturated stable spatial equilibrium). A stable spatial equilibrium in generation $\tau$ is called saturated if the total cell population stays within $1 \%$ of its maximal population size in generations $\{\tau-49, \tau-48, \ldots, \tau\}$. 


\subsubsection{The effects of the mortality regime on tumor growth}

Whether death is stochastic or deterministic and how long dead cells remain in the tumor strongly influence both the transient and saturated spatial equilibria. Figure 5.3 shows the spatial dynamics of a single run for matrix \#19 per scenario from Table 5.3. The results achieved from a single run can be representative for this matrix \#19, as we observe that the standard deviation over five runs for matrix \#19 is very small (see Appendix C.3, page 155 for details). By generation 200 for matrix \#19, the frequencies of cell types have

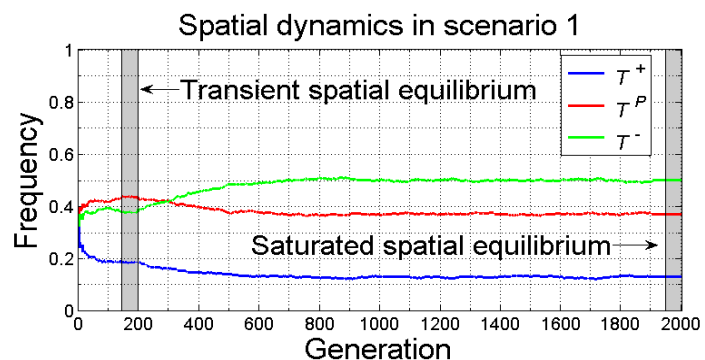

(a)

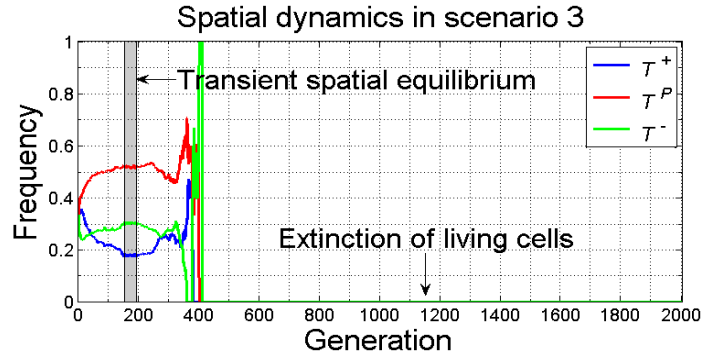

(c)

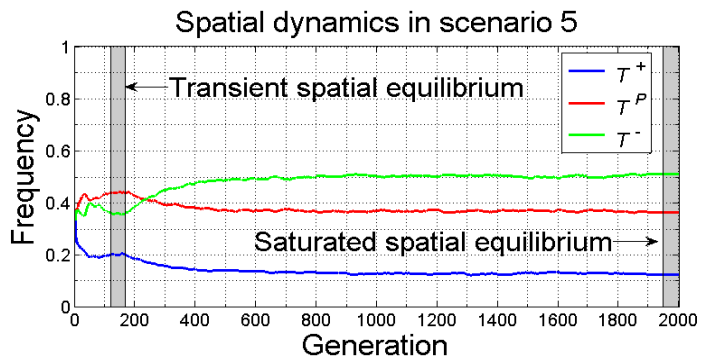

(e)

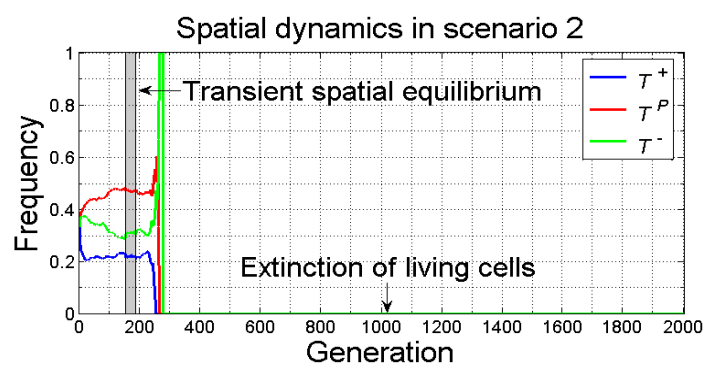

(b)

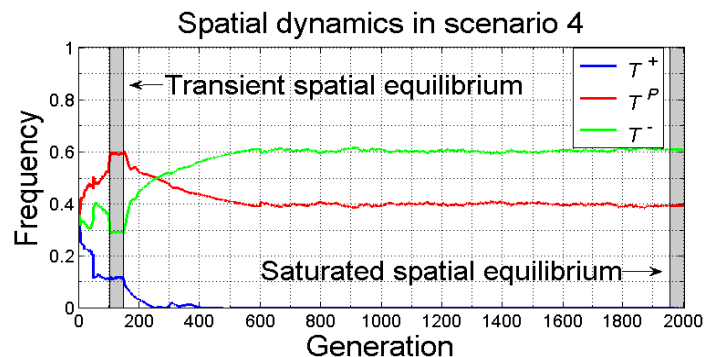

(d)

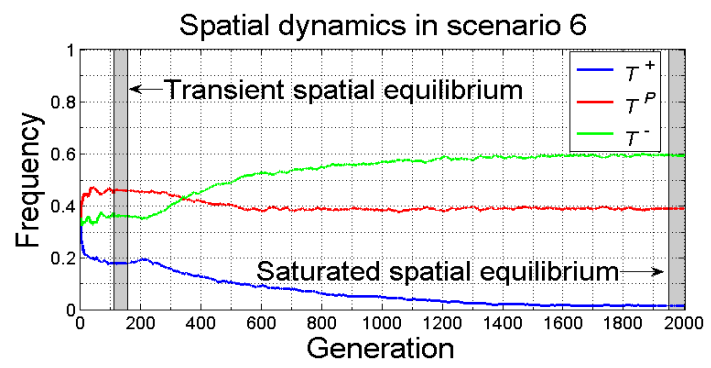

(f)

Figure 5.3: Transient and saturated equilibria corresponding to the six different mortality regimes (Table 5.3). This example uses payoff matrix \#19. The generations depicting stable transient/saturated equilibria are marked in gray. Scenarios 2 and 3 have no stable saturated equilibria as all cells die when saturation is reached. Each panel shows the frequencies of the three cell types over the course of 2000 generations of a single run.

reached transient equilibria in all scenarios even as the entire space has yet to be filled (Figure 5.4). In scenario 1 (mortality regime: stochastic death and removal of dead cells after 5 generations) we observe that $T^{+}, T^{P}$, and $T^{-}$cells are rather well mixed with dead cells and the tumor is tightly packed throughout. A similar tumor is observed in scenario 5 (mortality regime: deterministic death after 20 generations and removal of dead cells after 5 
generations) and scenario 6 (mortality regime: $5 \%$ stochastic death and removal of dead cells after 30 generations), while more dead cells are observed in the field in scenario 6 (with $\approx$ $60 \%$ cells dead) than in scenario 1 (with $\approx 20 \%$ cells dead) and scenario 5 (with $\approx 22 \%$ cells dead). In scenario 2 (mortality regime: deterministic death and no removal of dead cells) we see the formation of a necrotic core densely packed with dead cells. Scenario 3 (mortality regime: $5 \%$ stochastic death rate and no removal of dead cells) is similar to scenario 2, except that a small number of living cells remain appearing in the necrotic core. Eventually, each cell will die. In scenario 4 (mortality regime: deterministic death and removal of dead cells after 30 generations) the tumor becomes a ring with an outer surface of living cells, an inner surface of dead cells and bulges of living cells recolonizing an otherwise empty center.

Figure 5.5 illustrates the tumors at their saturated equilibria for the six scenarios using payoff matrix \#19. In scenarios 1 and 4 the living cells of the three types are relatively well mixed. In scenario 1 the entire space is filled. Dead cells are mixed with the living ones and the appearance is similar to the transient equilibrium. In scenario 4 the ring shape of the tumor progresses to a more patchy distribution of living and dead cells surrounding patches of empty space. Scenarios 2 and 3 (no removal of dead cells) result in the filling of the space with dead cells.

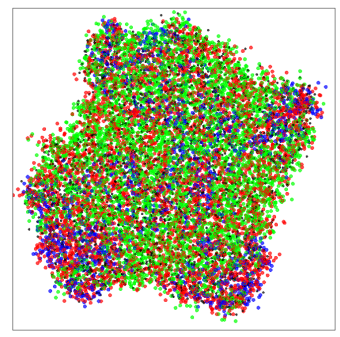

(a)

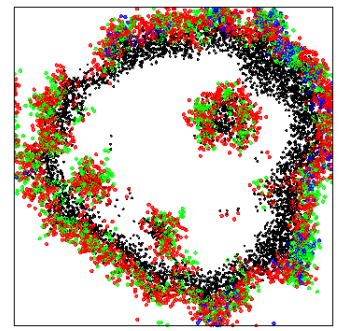

(d)

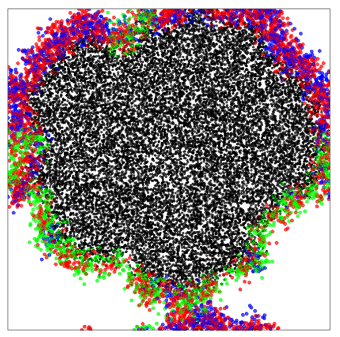

(b)

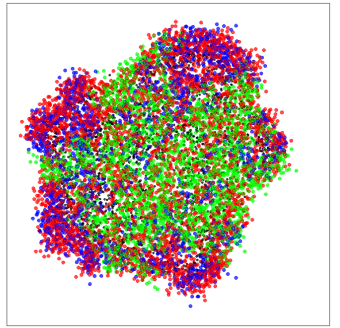

(e)

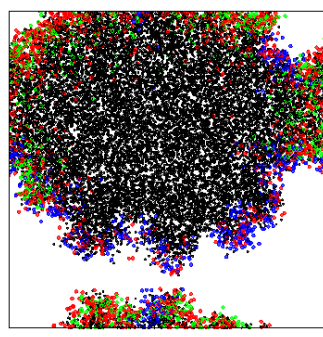

(c)

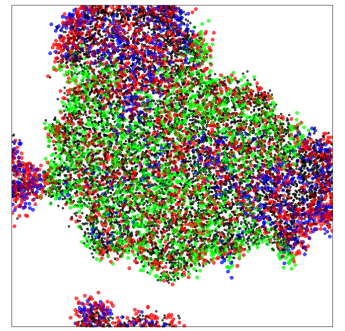

(f)

Figure 5.4: Tumors in space at the point where cell type frequencies achieve a stable transient equilibria. Subfigures (a), (b), (c), (d), (e) and (f) correspond to scenarios 1, 2, 3, 4, 5 and 6 in Table 5.3, respectively, with payoff matrix \#19. $T^{+}, T^{P}, T^{-}$and dead cells are denoted by blue, red, green and black color, respectively.

Tables C.1, C.2 and C.3 (see Appendix C.3, page 155) record the transient and saturated equilibrium frequencies of the three cell types, averaged over five simulations, for each of the six scenarios and for each of the 22 payoff matrices, respectively.

Based on the six scenarios and the twenty-two possible arrangements of payoff matrices, we observe the following: 


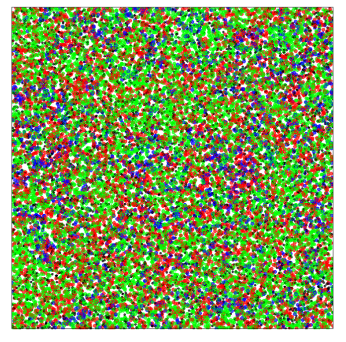

(a)

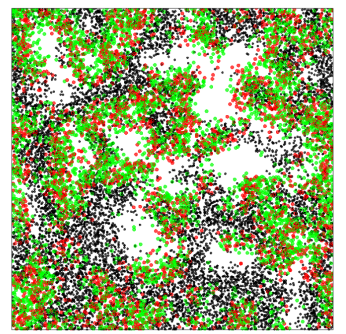

(d)

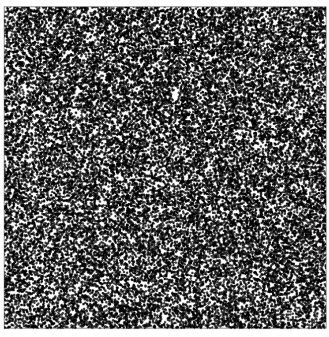

(b)

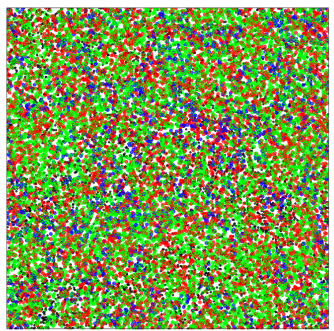

(e)

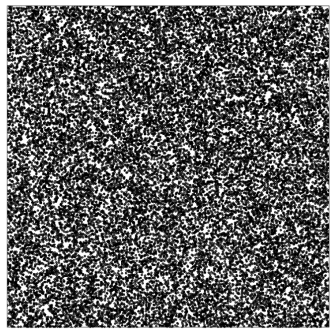

(c)

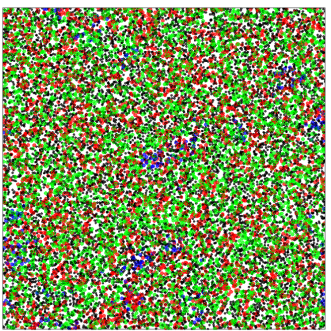

(f)

Figure 5.5: Tumors in space at the point where cell type frequencies achieve stable saturated equilibria. Subfigures (a), (b), (c), (d), (e) and (f) correspond to scenarios 1, 2, 3, 4, 5 and 6 in Table 5.3, respectively, with payoff matrix \#19. $T^{+}, T^{P}, T^{-}$and dead cells are denoted by blue, red, green and black color, respectively.

- In scenario 1 (mortality regime: 5\% stochastic death rate and removal of dead cells after 5 generations), the transient and saturated equilibrium frequencies for matrices from group I (positive) are very close to the matrix game ESS, while their equilibrium frequencies for matrices from group II (neutral) and III (negative) deviate from the matrix game ESS. In conclusion, when the ESS for the matrix game contains just two cell types $\left(T^{+}\right.$and $\left.T^{P}\right)$, the spatial model yields cell type frequencies near identical to this ESS. When the ESS of the matrix game contains all three cell types, the spatial game results in equilibrium cell type frequencies quite different than the nonspatial ESS.

- In scenario 2 (mortality regime: deterministic death after 20 generations and no removal of dead cells), the transient equilibrium frequencies for matrices from group I (positive) are close to the ESS while the transient equilibrium frequencies for matrices from group II (neutral) and III (negative) deviate substantially from the nonspatial ESS. For matrices in group II, $T^{-}$cells die out during the transient equilibrium, and the frequencies of $T^{+}$and $T^{P}$ cells converge on the 2-strategy nonspatial equilibrium if only $T^{+}$and $T^{P}$ exist. For the matrices in group III, the transient equilibrium frequency of $T^{-}$is much lower, and the frequencies of $T^{+}$and $T^{P}$ cells are higher than the nonspatial ESS. There is no stable saturated equilibrium as all cells die when the space is filled no matter which matrix is examined. This is due to the existence of the necrotic core, which keeps spreading and eventually takes over the entire field.

- In scenario 3 (mortality regime: $5 \%$ stochastic death rate and no removal of dead 
cells), the equilibrium frequencies are quite similar to those in scenario 2 . The necrotic core eventually takes over the space. While some living cells do survive for many generations, they eventually fail to proliferate as they become surrounded by dead cells. Eventually, all cells die.

- In scenario 4 (mortality regime: deterministic death after 20 generations and removal of dead cells after 30 generations), the transient equilibrium frequencies are quite similar to these in scenarios 2 and 3. For matrices from group I (positive) the frequencies of $T^{+}$and $T^{P}$ cells are close to the ESS ( $T^{-}$are absent from the ESS); for matrices from group II (neutral) $T^{-}$cells die off leaving a transient equilibrium with just $T^{+}$ and $T^{P}$ cells close to their 2 -strategy ESS. For matrices in group III, $T^{-}$cells persist but have much lower frequencies than predicted by the ESS. For all matrices and mortality regimes the transient phase sees a tumor that grows as an expanding ring. Living cells inhabit the outer edge of the ring and dead cells the inner edge. At the saturated equilibrium the thickness of the dead cells on the ring influences the spatial dynamics and cell type frequencies. A thinner ring of dead cells during the transient phase permits live cells to recolonize the empty space inside the ring, giving rise to a saturated equilibrium as shown in Figure 5.5d. A thicker band of dead cells results in a tumor at saturation that has large empty spaces and a relatively low number of living cells. The frequencies of cell types at the saturated equilibria for matrices from group I (positive) and matrices \#8, \#12 and \#15 deviate substantially from the nonspatial ESS as clumps of living cells become separated and patchy due to the large empty spaces within the tumor. The saturated equilibrium frequencies for the remaining matrices are close to a 2-strategy ESS, as either $T^{+}$or $T^{-}$dies out; $T^{P}$ cells occur at higher frequencies than expected by the ESS for all matrices in groups II and III.

- In scenario 5 (mortality regime: deterministic death after 20 generations and removal of dead cells after 5 generations), the transient equilibrium frequencies as well as the saturated equilibrium frequencies are very close to those generated from scenario 1.

- In scenario 6 (mortality regime: 5\% stochastic death rate and removal of dead cells after 30 generations), the transient and saturated equilibrium frequencies for matrices from group I (positive) are close to the ESS, while their equilibrium frequencies for matrices from group II (neutral) and III (negative) deviate from the ESS.

The mortality regime is paramount in determining tumor architecture. With no removal of dead cells (scenarios $2 \& 3$ ), the space becomes filled with dead cells only and the ultimate survival of the tumor would require unbounded space for living cancer cells to invade. When dead cells take a long time to disappear, the tumor exhibits rings of living cells surrounding necrotic regions, the tumor dies off (scenario 4), or dead cells represent over half of those visible in the tumor (scenario 6). The arrangement of these empty patches within the tumor becomes dynamic as some patches become colonized by living cells and new empty patches form. Regardless, the overall abundance of live cancer cells is much reduced because of 
the empty spaces and the long persistence of dead cells. If dead cells persist for only a short number of generations (scenario $1 \& 5$ ) the tumor grows and fills the entire space as a contiguous population of living cells. The living cells both expand along the margin of the tumor and continually repopulate the core of the tumor as cells die and disappear. The saturated equilibria in scenario 1 result in high and persistent populations of cancer cells; though the mosaic of cell types changes constantly at small spatial scales.

\subsubsection{Spatial vs. nonspatial dynamics}

To compare in more detail the dynamics and equilibria of the spatial versus nonspatial models, we consider the saturated equilibria for the 22 payoff matrices under just the first scenario (stochastic mortality and short duration before removing dead cells). This scenario produces a highly dynamic tumor that saturates at dense populations of living cells. We focus on the three important themes in tumorigenesis:

1. the population size of live cancer cells at the saturated equilibrium

2. the frequencies of cell types at the saturated equilibrium

3. the dispersion patterns of cell types within the tumor

To measure the dispersion pattern of a given cell type within the saturated tumor, we use the variance-to-mean ratio (Upton and Cook, 2014). The variance-to-mean ratio of type $i \in T$ is defined as

$$
\rho_{i}=\frac{\sum_{j=1}^{\Omega}\left(\omega_{i}^{j}-\bar{\omega}_{i}\right)^{2}}{(\Omega-1) \bar{\omega}_{i}}
$$

where the space is evenly divided into $\Omega=900$ subsquares. Varying the number of subsquares a bit gives us qualitatively similar results, therefore we consider $\Omega=900$ as being representative. Quantity $\omega_{i}^{j}$ is the number of type $i$ cells in the $j$-th subsquare and $\bar{\omega}_{i}$ is the average number of type $i$ cells per subsquare. The variance-to-mean ratio provides a quantitative measure of spatial dispersion or clumpiness (i.e., degree of aggregation of cells within certain regions of the field) (Tilman and Kareiva, 1997): A variance-to-mean ratio $\rho_{i}=1$ indicates that cells of type $i$ are randomly dispersed in space, a $\rho_{i}>1$ indicates a clumped dispersion, and a $\rho_{i}<1$ indicates an over-dispersed or more uniform dispersion in space.

For the 22 payoff matrices at saturated equilibria, Table 5.4 depicts the living population size, cell type frequencies, the variance-to-mean ratio for each cell type, and the ESSs for the nonspatial model. Results show the mean value for 5 runs. Standard deviations were very low and so we omit them from the table. In all runs, population sizes rose rapidly until the space was completely filled, usually after about 500 generations.

In terms of population size, there are only small differences between the 22 payoff matrices; though two subtle patterns are evident (Table 5.4). Matrix 2 of group I had 


\begin{tabular}{|c|c|c|c|c|c|}
\hline Group & $\#$ & $\begin{array}{l}\text { Saturated } \\
\text { equilibrium } \\
\text { population size }\end{array}$ & $\begin{array}{l}\text { ESS } \\
\left(T^{+}, T^{P}, T^{-}\right)\end{array}$ & $\begin{array}{l}\text { Saturated equilibrium } \\
\text { frequencies } \\
\left(T^{+}, T^{P}, T^{-}\right)\end{array}$ & $\begin{array}{l}\text { Variance-to-mean } \\
\text { ratio } \\
\left(T^{+}, T^{P}, T^{-}\right)\end{array}$ \\
\hline \multirow{12}{*}{ I (positive) } & 1 & 17746.4 & $(0.5455,0.4545,0)$ & $(0.5474,0.4526,0)$ & $(0.9559,1.0370,-)$ \\
\hline & 2 & 17837 & $(0.5455,0.4545,0)$ & $(0.5482,0.4518,0)$ & $(0.9867,1.0673,-)$ \\
\hline & 3 & 17772.4 & $(0.5455,0.4545,0)$ & $(0.5469,0.4531,0)$ & $(0.9709,0.9536,-)$ \\
\hline & 4 & 17754.6 & $(0.4545,0.5455,0)$ & $(0.4515,0.5449,0)$ & $(0.9615,0.9557,-)$ \\
\hline & 5 & 17763.4 & $(0.4545,0.5455,0)$ & $(0.4505,0.5495,0)$ & $(1.0550,0.9706,-)$ \\
\hline & 6 & 17773.2 & $(0.4545,0.5455,0)$ & $(0.4512,0.5488,0)$ & $(1.0489,0.9667,-)$ \\
\hline & 7 & 17778.8 & $(0.4000,0.6000,0)$ & $(0.3987,0.6013,0)$ & $(1.1149,0.9115,-)$ \\
\hline & 9 & 17718.4 & $(0.4000,0.6000,0)$ & $(0.3992,0.6008,0)$ & $(1.1116,0.9147,-)$ \\
\hline & 11 & 17809.2 & $(0.3333,0.6667,0)$ & $(0.3287,0.6713,0)$ & $(1.1676,0.8946,-)$ \\
\hline & 14 & 17788 & $(0.5455,0.4545,0)$ & $(0.5482,0.4518,0)$ & $(0.9586,1.0608,-)$ \\
\hline & 17 & 17790.4 & $(0.4545,0.5455,0)$ & $(0.4561,0.5439,0)$ & $(1.0111,0.9879,-)$ \\
\hline & 20 & 17817.6 & $(0.4000,0.6000,0)$ & $(0.3991,0.6009,0)$ & $(1.0572,0.8402,-)$ \\
\hline \multirow{4}{*}{ II (neutral) } & 8 & 17636.6 & $(0.3659,0.5659,0.0682)$ & $(0.3926,0.6069,0.0025)$ & $(1.1422,0.7932,7.8522)$ \\
\hline & 10 & 17779.2 & $(0.2856,0.6287,0.0857)$ & $(0.3280,0.6691,0.0029)$ & $(1.1337,0.8275,9.3954)$ \\
\hline & 12 & 17730 & $(0.4516,0.4149,0.1290)$ & $(0.5419,0.4532,0.0050)$ & $(1.0083,1.1209,8.6381)$ \\
\hline & 15 & 17747.4 & $(0.4074,0.5185,0.0741)$ & $(0.4494,0.5453,0.0053)$ & $(1.0836,0.9507,9.2382)$ \\
\hline \multirow{6}{*}{ III (negative) } & 13 & 17547.4 & $(0.3333,0.3333,0.3333)$ & $(0.3324,0.3368,0.3308)$ & $(2.0569,1.6380,2.2931)$ \\
\hline & 16 & 17596.6 & $(0.2800,0.4000,0.3200)$ & $(0.2506,0.4123,0.3371)$ & $(2.9114,1.5339,2.5050)$ \\
\hline & 18 & 17524.8 & $(0.2500,0.5000,0.2500)$ & $(0.2518,0.5151,0.2327)$ & $(2.5338,1.1878,3.0966)$ \\
\hline & 19 & 17427 & $(0.1667,0.3667,0.4667)$ & $(0.1342,0.3638,0.5021)$ & $(3.1905,1.3738,1.3250)$ \\
\hline & 21 & 17451 & $(0.1667,0.5834,0.2500)$ & $(0.1525,0.6020,0.2455)$ & $(3.4840,0.9954,2.4489)$ \\
\hline & 22 & 17484.4 & $(0.2016,0.6285,0.1699)$ & $(0.2218,0.6469,0.1313)$ & $(2.7141,0.7627,4.4589)$ \\
\hline
\end{tabular}

Table 5.4: Saturated equilibrium population sizes (averaged over five runs), ESSs, saturated equilibrium frequencies (averaged over five runs) and variance-to-mean ratios (averaged over five runs) for each of the 22 matrices

the highest mean of 17837 living cells, and matrix 19 of group III had the lowest mean of 17427; a mere $2.3 \%$ difference.

We observe the following:

- Tumors from group III (negative) reach slightly smaller population size when the frequency of $T^{-}$is greater than 0.1 . There is also a small and negative correlation between the clumping of cell types (high variance-to-mean ratios) and their population size. We conclude that a smaller population size results because tumors with high $T^{-}$frequencies exhibit a clumped dispersion. This clumping of all cell types reduces proliferation rates from like cell types interacting.

- Population sizes are relatively large for matrices from group I where only $T^{+}$and $T^{P}$ coexist. These tumors have near random dispersion patterns by cell type; thus eliminating non-random, positive assortative interactions by cell type. Matrices from group II (low frequency of $T^{-}$) have population sizes close to those of group I. In group II, only $T^{-}$cells exhibit substantial clumping; but they are too few to impact the tumor's overall population size.

Many of the payoff matrices result in large differences between the cell type frequencies in the spatial model as compared to the nonspatial ESS (Table 5.4) We highlight 2 results:

- Spatial dynamics converge to the ESS for all of the matrices in group I. With these matrices only $T^{+}$and $T^{P}$ persist in both replicator and spatial dynamics. 
- For matrices in groups II and III, the rarest cell type in the ESS suffers and equilibrates at a lower frequency than predicted by the ESS. For matrices in group II (neutral), $T^{-}$does much worse than at the ESS. Also, a highly clumped dispersion occurs for $T^{-}$cells for these matrices. For matrices \#16, \#19 and \#21 of group III, $T^{+}$cells do much worse than predicted by ESS. In these cases, the $T^{+}$cells have a highly clumped dispersion.

Figure 5.6 shows examples of different saturated equilibria with different levels of clumping. For matrix \#7 (group I), we see the spatial population converging to the ESS with a random dispersion of both $T^{+}$and $T^{P}$ cells (Figure 5.6a). The tumors become quite well mixed for all saturated equilibria from group I payoff matrices. For matrices \#19 and \#22, respectively, the cell type that does worst relative to the ESS has the highest variance-tomean ratio at the saturated spatial equilibria (Figures 5.6b and 5.6c). This result holds for all matrices in groups II (neutral) and III (negative).

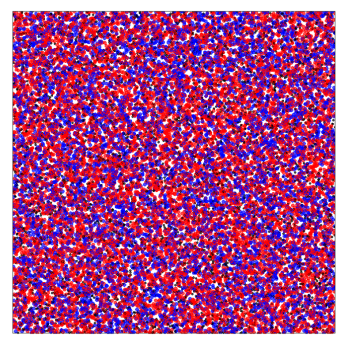

(a)

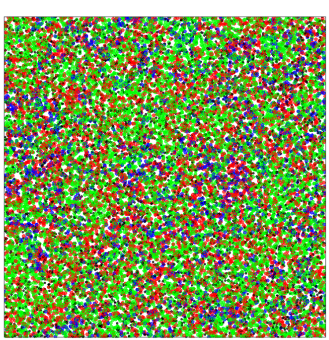

(b)

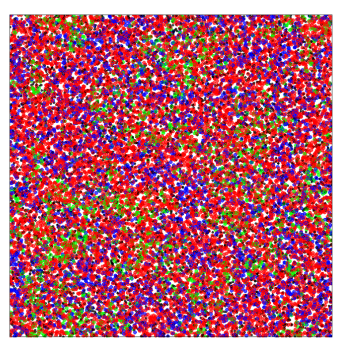

(c)

Figure 5.6: Snapshots of the field at saturated equilibria when (a) spatial dynamics converge to the ESS, (b) $T^{+}$does worse in space than it does at the ESS, and (c) $T^{-}$does worse in space than it does at the ESS. $T^{+}, T^{P}, T^{-}$and dead cells are denoted by blue, red, green and black color, respectively.

Whether cell clumping is observed or not seems to depend on the particular payoff matrix. No cell clumping happens for the matrices in group I, in which ESSs have no $T^{-}$cells. In the spatial model, when $T^{-}$cells die out, $T^{+}$and $T^{P}$ cells can only proliferate by interacting with each other, because the diagonal of each payoff matrix is 0 . We conclude that the inter-cell type facilitation leads to the two cell types becoming well mixed with each other. Cell clumping (either $T^{+}$or $T^{-}$cell clumping) is observed for matrices in groups II and III, for which ESSs have three types present. Interestingly, across all matrices, $T^{P}$ always shows low levels of clumping. Its dispersion is always near random, or for some matrices, over-dispersed. In the first 10 generations, all cell types exhibit a clumped dispersion, due to the small dispersal radius. However, $T^{P}$ cells always grow fast and spread rapidly across the field, because $T^{P}$ cells enjoy an initially high average payoff when compared to other types, i.e., $\frac{1}{3}(c+d) \geq \frac{1}{3}(a+b) \geq \frac{1}{3}(e+f)$ or $\frac{1}{3}(c+d) \geq \frac{1}{3}(e+f) \geq \frac{1}{3}(a+b)$. The spread of $T^{P}$ cells provides a higher probability of interacting with the other two cell types. As a result, $T^{P}$ cells keep growing and spreading. Eventually, $T^{P}$ cells become randomly or over-dispersed throughout the tumor. 


\subsection{Effects of the frequency-dependence radius, the dispersal radius and the density-dependence radius on spatial equilibria}

In this section we investigate the effects of independently varying the frequency-dependence, dispersal and density-dependence radius on the eco-evolutionary dynamics of the spatial model. The spatial game will be analyzed with matrices \#7 (group I; no $T^{-}$cells in the ESS), \#8 (group II; low frequency of $T^{-}$cells in the ESS), and \#22 (group III; high frequency of $T^{-}$cells in the ESS) as these are typical representatives of their groups. The results are averaged over five runs for each of these matrices. However, the variance in these runs is very small.

\subsubsection{Effect of the frequency-dependence radius}

We compared the outcomes of the spatial game when the frequency-dependence radius was set to $0.5,1,10$, and 50, while holding the dispersal and density-dependence radii to 1 . Regardless of the frequency-dependence radius, $T^{-}$cells die out for the group I matrix \#7 (Figure 5.7b). For group II matrix \#8 and group III matrix \#22 the frequencies of $T^{-}$ approach their nonspatial ESS values as the frequency-dependence radius increases (Figure 5.7b).

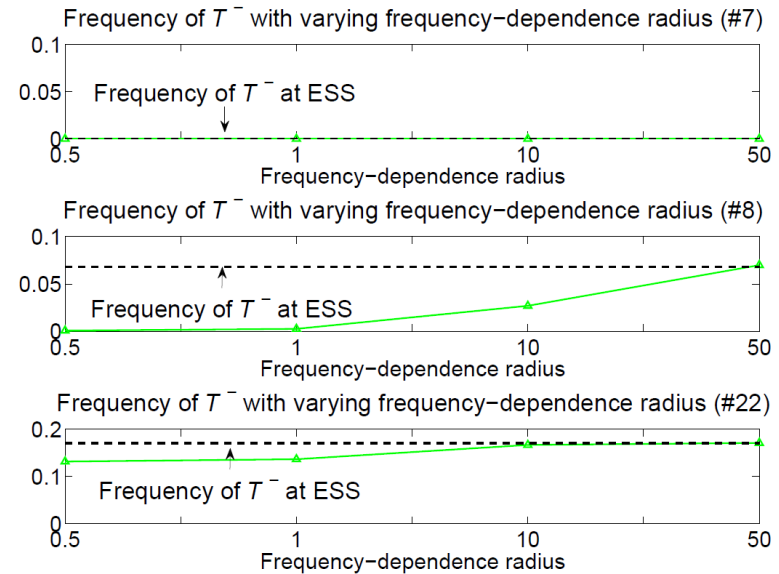

(a) Frequency of $T^{-}$

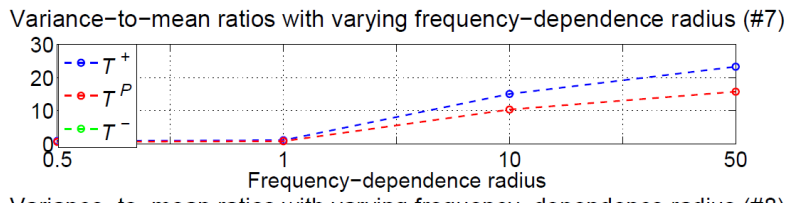

Variance-to-mean ratios with varying frequency-dependence radius (\#8)

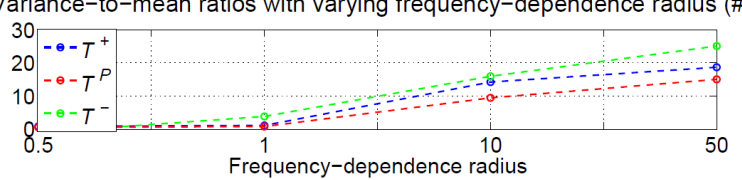

Variance-to-mean ratios with varying frequency-dependence radius (\#22)

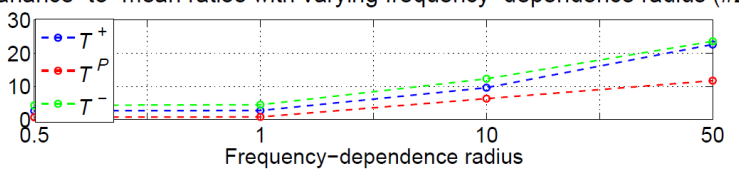

(b) Variance-to-mean ratios

Figure 5.7: Effect of the frequency-dependence radius. (a) Saturated equilibrium frequency of $T^{-}$cells for matrices \#7, \#8 and \#22. (b) Saturated equilibrium variance-to-mean ratios of all types for matrices \#7, \#8 and \#22.

Interactions become random with respect to cell type once the frequency-dependence radius encompasses the entire field. When this happens the equilibrium of the spatial model must converge on the ESS of the nonspatial model: the probability of a focal cell interacting with a neighbor of type $j \in T$ equals the overall frequency of type $j$. The saturated equilibrium frequencies are very close to the ESS (with maximal difference \pm 0.001 ). As 
the frequency-dependence radius increases all cell types exhibit an increasingly clumped dispersion (Figures 5.7 and 5.13). The variance-to-mean ratio increases with the frequencydependence radius because the game now involves distant cells even as daughter cells remain close together.

\subsubsection{Effect of the dispersal radius}

We compared the outcomes of the spatial game when the dispersal radius was set to 0.5 , 1,10 , and 50, while holding the frequency-dependence and density-dependence radii at 1 . Like increasing the frequency-dependence radius, increasing the dispersal radius results in a convergence of the cell type frequencies on the ESS (Figure 5.8b). Moreover, when the dispersal neighborhood covers the entire field (dispersal radius of 50), the spatial equilibrium frequencies are nearly identical to their ESS (with difference \pm 0.001 ). Increasing the dispersal radius reduces the variance-to-mean ratio for all cell types. A high dispersal radius disperses daughter cells widely and creates a within cell type dispersion pattern that is random or even over-dispersed (Figures 5.8b and 5.13).

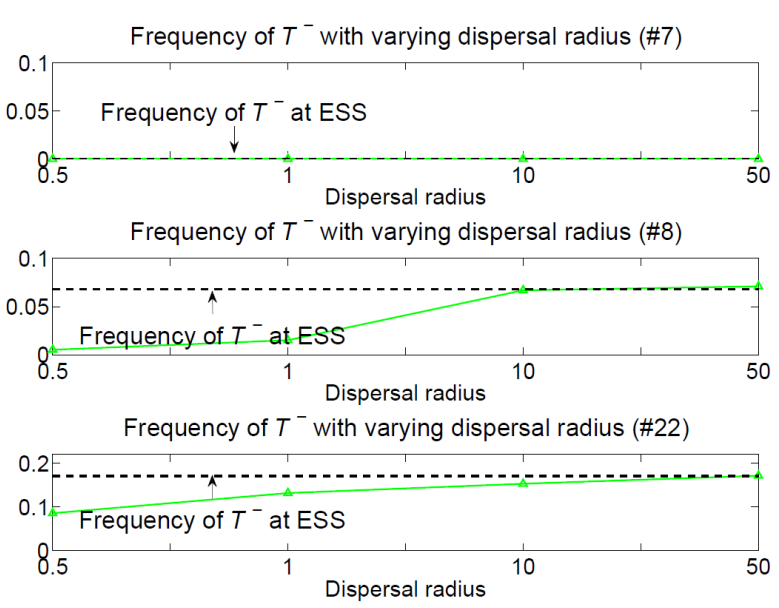

(a) Frequency of $T^{-}$

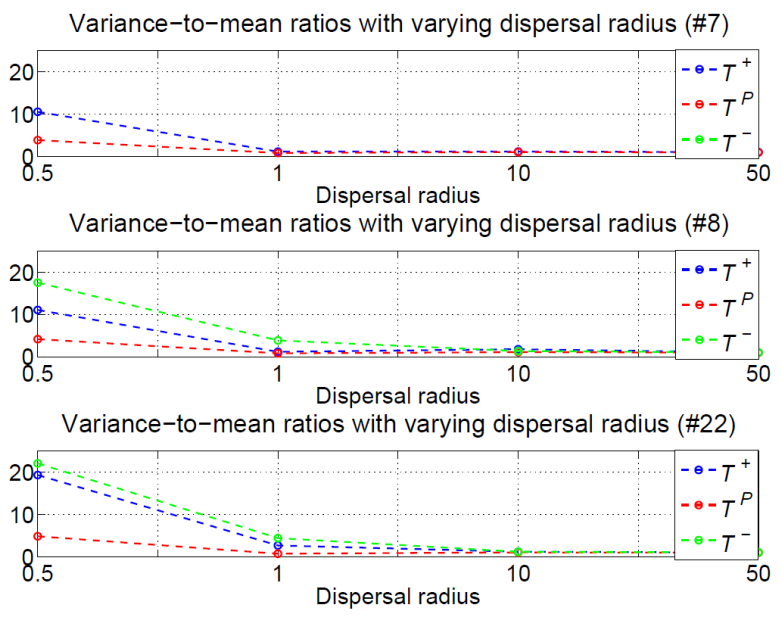

(b) Variance-to-mean ratios

Figure 5.8: Effect of the dispersal radius. (a) Saturated equilibrium frequency of $T^{-}$for matrices \#7, \#8 and \#22. (b) Saturated equilibrium variance-to-mean ratios of all types for matrices \#7, \#8 and \#22.

\subsubsection{Effect of the density-dependence radius}

We compared the outcome of the spatial game when the density-dependence radius is set to $0.5,1,10$, and 50, while holding the frequency-dependence and dispersal radii at 1 . The frequencies of cell types in the saturated community converge to the ESS as the densitydependence radius increases. Group I matrix \#7 leads to no $T^{-}$cells in the field. For group II matrix \#8 and group III matrix \#22 the frequencies of $T^{-}$approach their nonspatial ESS values as the density-dependence radius increases (Figure 5.9b). We observe very high levels of clumping by cell type when the density-dependence radius is 10 and 50. Curiously, 
a density-dependence radius of 10 results in a higher variance-to-mean ratio than a densitydependence radius of 50 (Figure $5.9 \mathrm{~b}$ ). When the density-dependence radius is low the tumor
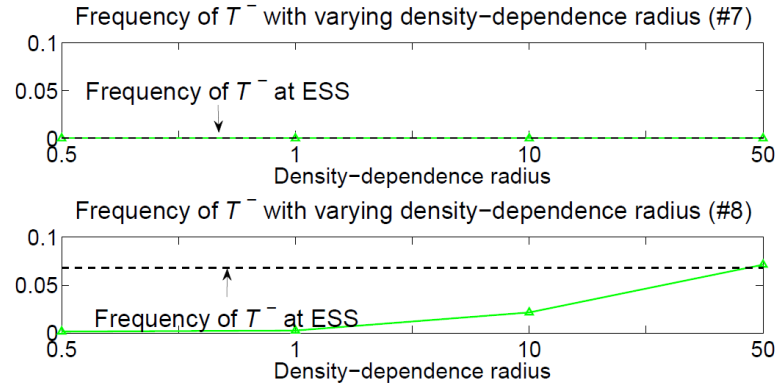

Frequency of $T^{-}$with varying density-dependence radius (\#22)

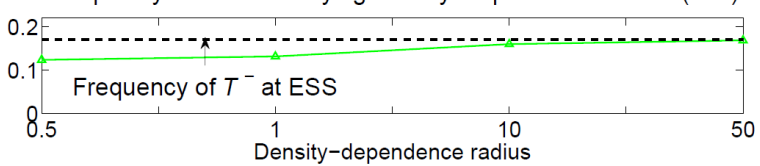

(a) Frequency of $T^{-}$

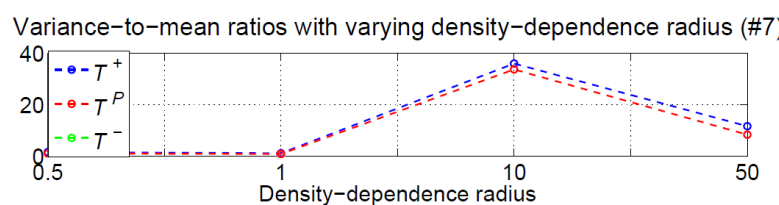

Variance-to-mean ratios with varying density-dependence radius (\#8)

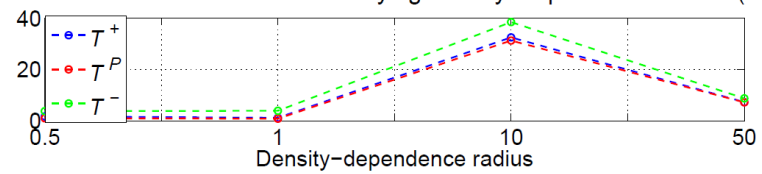

Variance-to-mean ratios with varying density-dependence radius (\#22)

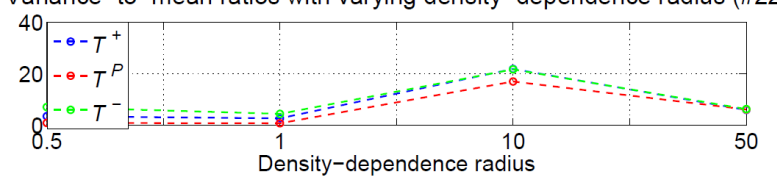

(b) Variance-to-mean ratios

Figure 5.9: Effect of the density-dependence radius. (a) Saturated equilibrium frequency of $T^{-}$for matrices \#7, \#8 and \#22. (b) Saturated equilibrium variance-to-mean ratios of all types for matrices \#7, \#8 and \#22.

expands to fill the entire space (Figure 5.11a). At a high density-dependence radius of 10 the tumor becomes a number of densely packed clusters with empty spaces between these clusters (Figure 5.10b). Each cluster has a very high density in the interior and a much lower density at its exterior. With a density-dependence radius that encompasses the entire space $(=50)$, we observed one large cluster of cells (Figure 5.10c). A large density-dependence radius permits cells to proliferate rapidly and for prolonged periods. It takes longer for density limitations to be reached. Yet, the low dispersal radius causes cells to bunch up as clusters $(=10$; Figure $5.10 \mathrm{~b})$ or as a single cluster $(=50$; Figures $5.11 \mathrm{c}$ and 5.13$)$.

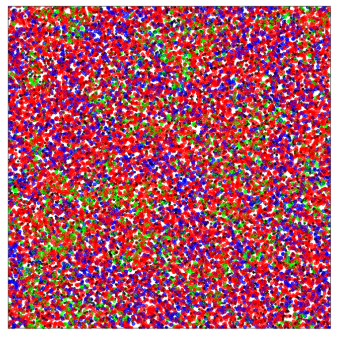

(a)

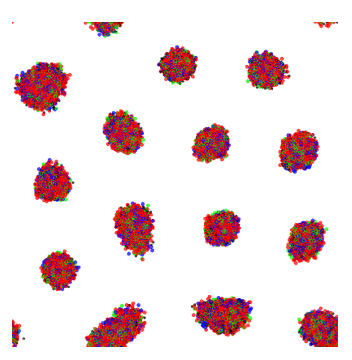

(b)

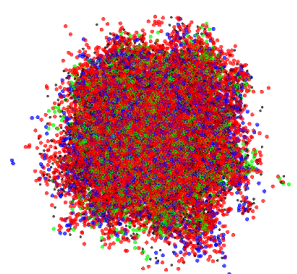

(c)

Figure 5.10: The appearance of the simulated tumors for matrix \#22 when the densitydependence radius is set to: (a) 1, (b) 10, and (c) 50. Each figure is at saturated equilibrium following 2000 generations. 


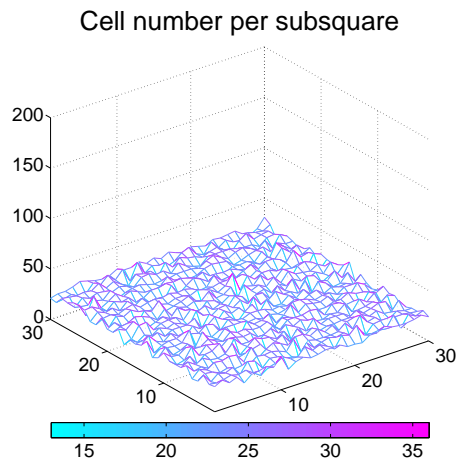

(a)

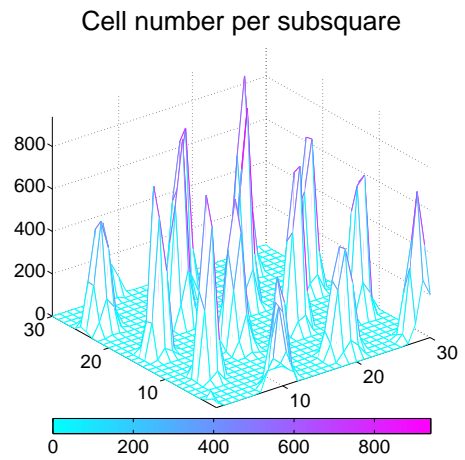

(b)

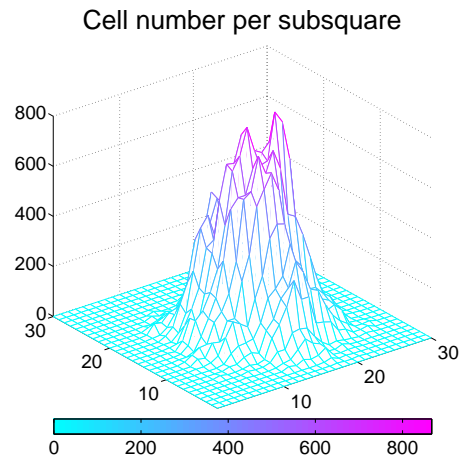

(c)

Figure 5.11: Cell numbers per subsquare of the simulated tumors for matrix \#22 when the density-dependence radius is set to: (a) 1, (b) 10, and (c) 50. These cell density distributions correspond to the panels of Figure 5.10.

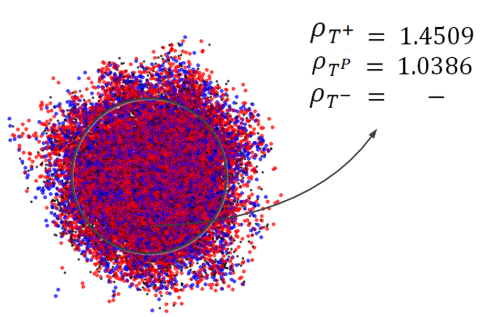

(a)

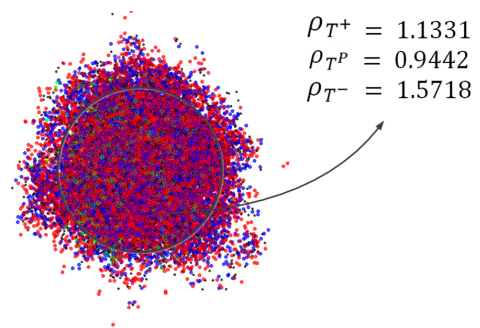

(b)

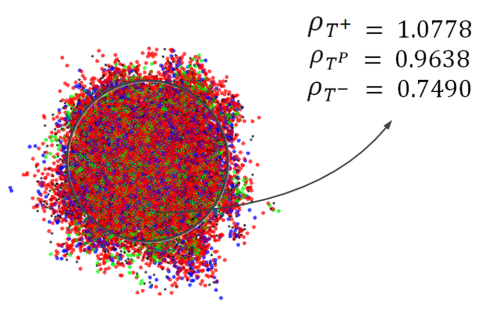

(c)

Figure 5.12: Simulated tumors after 2000 generations for matrices (a) \#7, (b) \#8, and (c) \#22. The density-dependence radius has been set to 50, thus resulting in densely packed tumors. Furthermore, there are varied levels of clumping by cell type and by matrix as shown by the variance-to-mean ratios. For matrix $\# 8$ and $\# 22$, the $T^{-}$cells are strongly clumped and over-dispersed, respectively.

\subsection{Conclusion \& discussion}

We used an agent-based, spatially explicit model to study tumor dynamics as an evolutionary game. The individual cancer cells represent the players, three cell types represent their strategies, and interactions between cells result in payoffs that influence a given cell's proliferation rate. We used a continuous space model meaning that cancer cells can occupy any point in the space. We included density-dependent effects where limited space and resources place upper bounds on the number and density of cancer cells inhabiting the resulting tumor. We included frequency-dependent effects by having three cell types. The proliferation rate of a given cancer cell is influenced by its type and the cell types around it. The tumor itself grows as daughter cells disperse some distance from proliferating cells.

The model is intended for metastatic castrate-resistant prostate cancer where up to three different cell types may coexist within the tumor: $T^{+}$(cells with androgen receptors and requiring an external source of testosterone), $T^{P}$ (cells capable of producing their own testoster- 


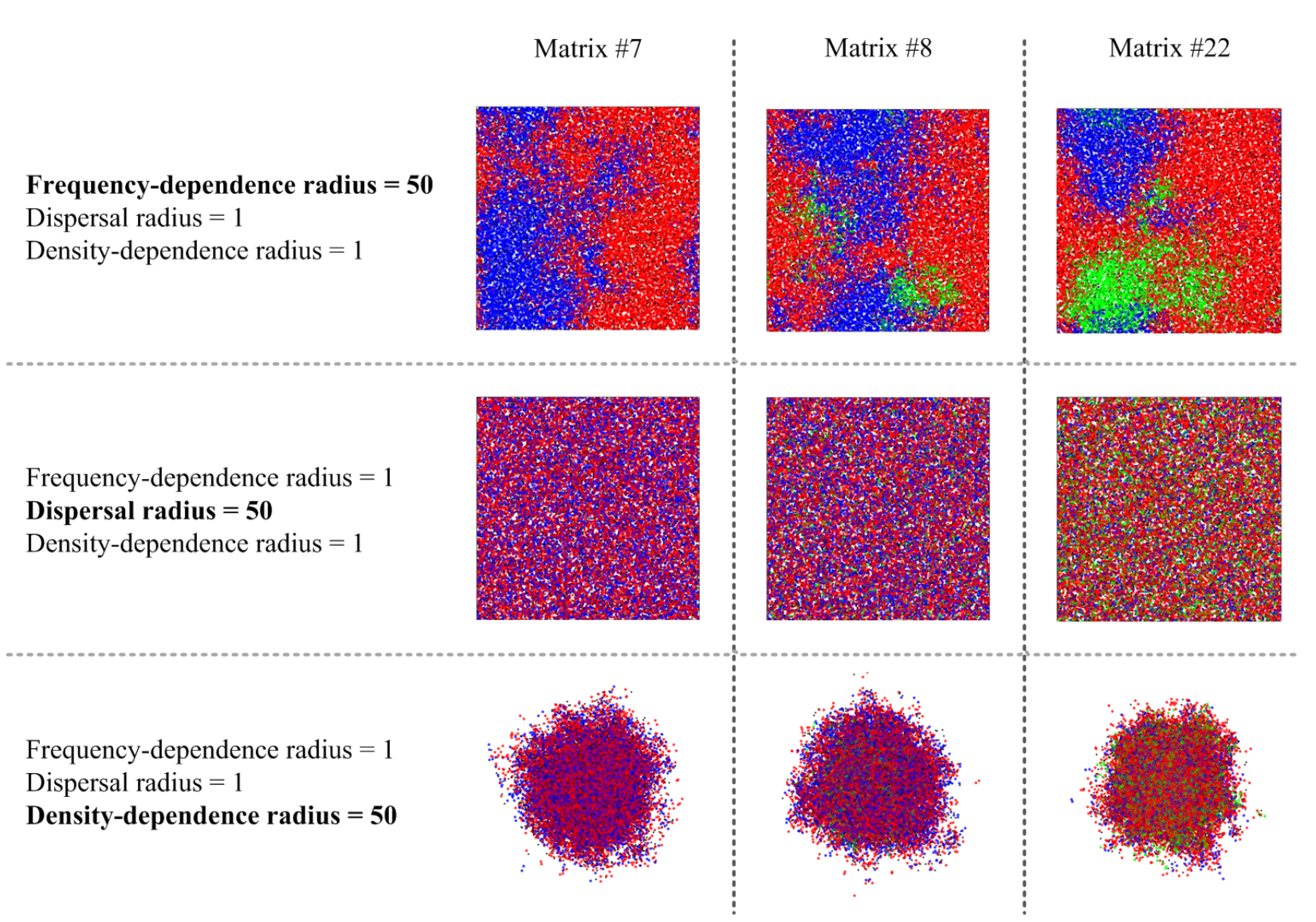

Figure 5.13: Simulated tumors resulting from setting either the frequency-dependence, dispersal, or density-dependence radius to the highest level (shown as the rows) while holding the other two at 1 . The columns represent different payoff matrices, and the simulations were run for 2000 generations insuring a saturated equilibrium.

one, some of this becomes publicly available to other cells), and $T^{-}$(cells that lack androgen receptors, and neither synthesize nor require testosterone). We extend a matrix game model based upon these three cancer cell types and their known biology (Zhang et al., 2017). Of interest in the modeling is the resulting success and frequency of the $T^{-}$cells as this likely relates to the success of subsequent therapy.

Advanced prostate cancer generally metastasizes to the bone and may eventually form tumors in one to over a dozen locations within the patient's skeleton. Such tumors seem to be largely composed of $T^{+}$cells that respond well to anti-androgen therapy. But, therapy may fail and the tumor progresses to metastatic androgen resistance. This may occur through the emergence of $T^{P}$ cells and/or $T^{-}$cells. By producing testosterone, $T^{P}$ cells "rescue" the $T^{+}$cells and may promote their resurgence within the progressing cancer. The next line of therapy (e.g., abiraterone) targets the mechanisms used to create testosterone. If $T^{-}$cells are absent to rare then such therapy should show success, but if $T^{-}$cells form a sizeable portion of the tumor, then such patients will be non-responders and the targeted therapy may fail immediately. The underlying matrix game can take on 22 distinct forms based on the rank-ordering of payoffs within the matrix. Interestingly, 12 of these show an absence of $T^{-}$at the ESS, 4 show a low frequency of $T^{-}$cells coexisting with $T^{+}$and $T^{P}$ cells, while 6 show a high frequency of $T^{-}$cells at the ESS. 
Our model is intended to make several advances and contributions to spatially explicit models of tumor growth. First, we consider different mortality regimes that result in substantially different patterns of tumor growth. In comparing scenarios corresponding to different mortality regimes we see distinctive transient and saturated equilibria. Second, we compare the saturated equilibria frequencies of cell types in the spatial game with the ESS of the nonspatial matrix game. Third, we vary the neighborhood size over which densitydependent and frequency-dependent effects can occur - such a case study had not yet been performed with spatial models. Fourth, we can vary the dispersal distance of daughter cells - this is an important component of what have been termed "grow-or-go" spatial models of tumor growth where cancer cells are presumed to have a trade-off between proliferation rate and dispersal ability (Gallaher and Anderson, 2013).

We ran six scenarios corresponding to mortality regimes representing extremes of stochastic mortality rates versus fixed cell lifespans, and rapid decomposition versus no decomposition of dead cells. With a fixed lifespan, the tumor grows outwards as a ring leaving behind a core of dead cells that eventually decompose to leave an empty interior. This mimics the formation of a necrotic core as seen in many tumors. A ring of dead cells forms a barrier that retards the proliferation of nearby living cells. But, eventually some daughter cells cross into the space left by the now decomposed dead cells. The living cells that cross the barrier create their own smaller rings of proliferation and mortality that maintain various empty "necrotic" spaces. These successive generations of proliferation and death create an ever changing mosaic of rings of living cells, dead cells and empty spaces. While we did not explore this in detail, it presents an intriguing simulation applicable to real tumors where the necrotic regions are not static but subject to recolonization. When dead cells never decompose and simply accumulate the simulated tumor at first grows outwards even as a necrotic core forms. In this case the necrotic region becomes crowded with dead cells and can never be recolonized by living cancer cells. In the absence of continued expansion, the tumor would eventually run out of space and becomes a mass of dead cells. What of actual tumors? A fairly regular turnover of cells within the tumor via cell births and deaths and a rapid decomposition of dead cells are likely the norm (Gordon, 2003; Nathan, 2006). Necrotic regions likely occur not because of fixed life spans of cancer cells, but because of tumor heterogeneity in other properties such as blood flow, oxygen and $\mathrm{pH}$.

With respect to the frequency of cancer cell types, we examined the transient and saturated equilibria of the model tumors. We observe an interesting difference between these two equilibria, which corresponds to actual tumors. Prostate cancer at an early stage, i.e., in our case in a transient phase, is likely more treatable as the number of $T^{-}$cells remains low. Ideally, treatment should start at this early stage and this makes studying spatial dynamics at a transient phase important. Yet, diagnosis or therapy may not occur until a saturated equilibrium has occurred where a given tumor has reached a large size and become less treatable.

In our spatial model, the saturated equilibrium frequencies of cell types frequently deviated from the nonspatial ESS, and such discrepancies vary with the 22 possible payoff matrices. Matrices with an ESS of just two cell types show no discrepancies between the 
spatial and nonspatial models. When the ESS includes all three cell types, the rarest strategy at the ESS (generally the $T^{+}$or $T^{-}$cell type) tends to suffer in the spatial game and exhibit a lower steady-state frequency than predicted by the nonspatial ESS. In fact, for some matrices with very low $T^{-}$frequency at the nonspatial ESS, $T^{-}$goes extinct for most replicates of the simulations. Clumping or kin effects explain this property of the spatial model. With a relatively small dispersal radius, cell types become clumped and hence a cell type interacts with its own type more than would be expected by chance. This is a standard property of many spatial models, and in fact can promote the evolution of cooperation (Ohtsuki et al., 2006). But here, this matrix model of prostate cancer "punishes" like interacting with like, and so positive assortative interactions reduce proliferation rates. The more clumped a cell type, the greater this disadvantage. With just two cell types, there is little clumping. With three cell types, all else equal, the rarer cell types become more clumped, and $T^{-}$seems to become more clumped than either $T^{P}$ or $T^{+}$.

The clumping of cell types observed in our spatial model has significance for drawing inferences from the distribution of different cell types within actual tumors. The spatial segregation of cell types sometimes observed in actual tumor biopsies may indicate underlying heterogeneity in blood vasculature, $\mathrm{pH}$, or position with respect to the edge or interior of the tumor (Mansury et al., 2002; Friedl and Wolf, 2003). But, as in our model, it may not indicate any underlying habitat structure within the tumor. The clumped distribution of cancer cells by type may simply reflect limited dispersal of daughter cells following proliferation. As expected, increasing the dispersal radius eliminates clumping and results in a convergence of the spatial model's cell-type frequencies with those of the nonspatial ESS. Complete convergence occurs when the dispersal radius encompasses the entire space as well.

While a small dispersal radius promotes clumping, a small frequency-dependence radius acts against clumping as like interacting with like suppresses proliferation. The emergent level of clumping reflects these opposing forces. Like the dispersal radius, increasing the frequency-dependence radius results in convergence of the spatial model's cell type frequencies to those of the nonspatial ESS - but with an important caveat. Increasing the frequencydependence radius actually results in extensive clumping by cell type, sometimes resulting in the near perfect segregation of cell types in space. When the frequency-dependence radius encompasses the entire tumor, then cell-cell interactions occur at random, regardless of clumping. Hence, frequency interactions no longer counterbalance the clumping caused by a limited dispersal radius. Our results highlight to the need to pay more attention to the interplay between the distance over which cells disperse and the neighborhood size over which frequency-dependent interactions take place. For instance, in grow-or-go models dispersal occurs at a larger scale than cell-cell interactions. The converse happens in models where a cell experiences the collective or diffuse actions of a large number of perhaps distant neighbors. Measuring or inferring the spatial scale of dispersal versus cell-cell interactions within actual tumors from biopsies presents both an opportunity and a challenge.

In our spatial model, we could independently vary the radius at which tumor cells experience the negative effects of competition from neighbors and the radius at which cells interact in a frequency dependent manner based on their type. Whereas increasing the dis- 
persal radius and the frequency-dependence radius causes the cell type frequencies of the spatial model to converge to those of the nonspatial ESS, increasing the density-dependence radius merely causes incomplete convergence. Increasing the density-dependence radius relative to the dispersal radius results in different dispersion patterns of cells regardless of type. When smaller than the dispersal radius, the cancer cells become almost uniformly dispersed in space. When the density-dependence and dispersal radii are equal, the cancer cells are essentially randomly dispersed. When the density-dependence radius is ten times the dispersal radius, the model no longer produces a continuous tumor spread across the space. Instead, the space is occupied by clusters of smaller tumors. The small dispersal radius promotes clumping. The empty spaces between the micro-tumors remain because of the long-distance suppression of proliferation by the densely packed cells of the clusters. Finally, when the suppressive effects of the cancer cells on each other's proliferation rates span the entire space, then the tumor becomes a very dense single mass that does not expand to fully fill the space. The dispersal radius keeps cells clumped while the space-wide suppression of proliferation prevents expansion beyond the boundaries of the tumor mass into the empty space.

Spatial models that have been introduced in the context of evolutionary game theory are usually confined to spatially explicit structures, such as graphs or lattices, including fields composed of identical hexagonal or square cells. In these models, individuals are represented as vertices of the graph or cells in a regular field. Individuals usually interact with their immediate neighbors and their payoffs and strategies depend on and evolve with these interactions. The simplest forms of such models were originally adopted to study evolution of social behaviors (Nowak and May, 1993). Early models had no births and deaths. Later ones included additional interactions, reproduction, and death rules to study evolution (Gerlee and Anderson, 2007; Simpson et al., 2007; Reichenbach et al., 2007). Empty vertices, created when cells die or move and limitations on cells growth were included in these models as well. While such models are more general than the original ones, they are still limited by assumptions on the structure of the field and definitions of the neighborhood. In this paper we went beyond rigid spatial models, by putting forward a continuous-space model of tumorigenesis. The advantage of using such a model is the flexibility of the continuous space and of the action rules. In our previous work we have shown how varying a fixed number of neighbors majorly impacts the predictions of grid models (Abrudan et al., 2016). Moreover, the continuous-space models seem to be more appropriate for modeling cancer where tumor cells may occur throughout a space and at very different local densities. Even though more rigid spatial models can be computationally efficient, this efficiency decreases rapidly when population sizes become large or when the radii of density-dependence, frequencydependence, and dispersal increase. Our implementation of the continuous-space model is efficient. A simulation running on a standard computer cloud takes a matter of minutes, independent of population or interaction radii size.

In summary, we have shown that spatially explicit evolutionary models often provide outcomes that differ from those in nonspatial ones. This, together with the spatial character of real tumors, suggests that space is a key element of tumorigenesis. Moreover, we have shown that continuous-space models are appropriate for modeling tumor growth, as they 
allow for flexibility of interaction rules and the spatial scales crucial to cell proliferation. In this work, we show how the scale at which cancer cells disperse and experience frequencyand density-dependent processes strongly influences the frequencies and dispersion patterns of cell types within the tumor. As a result, we have discovered various distinct cell dispersion patterns in space, such as near complete cell segregation, random cell dispersion, and overdispersion. Furthermore, the scale at which density-dependent processes operate can alter tumor architecture and create continuous masses of cells, separate clusters of cancer cells, and dense tumor masses surrounded by empty space. Some of our results accord with clinical and laboratory observations and others may help in the further development of spatial evolutionary models of cancer.

Our model and results invite future research. First, the scenario in which rings of dead cells form needs further and more detailed analysis, as results from such scenarios resemble real tumors for many cancer types. Second, the spatial model can be expanded to include blood vasculature and the immune system to determine tumor growth and heterogeneity. Third, the model can be used to test various regimes of cancer treatment. Of particular interest are therapies such as adaptive or double-bind therapies (Gatenby et al., 2009a,b). 


\section{Conclusions}

In this thesis we have proposed several game-theoretical spatial and nonspatial models for studying biological interactions in three diverse areas: predator-prey interactions among mites, competition among microbial species, and tumorigenesis in prostrate cancer involving three types of cancer cells (Chapters 2-5). For each of these three problems, we selected a different model which we felt described the problem best, we analyzed their dynamics with respect to several settings and provided reasons for differences in their predictions.

This chapter concludes the thesis. In Section 6.1 we will briefly present our results as answers to the research questions posed in Section 1. In Section 6.2 we will discuss limitations of the research of this thesis and possibilities for future research steps.

\subsection{Answers to research questions}

Chapter 2 focused on modeling summer-season interactions between the predatory mites (Acari: Phytoseiidae) and the fruit-tree red spider mites (Acari: Tetranychidae) as a nonspatial game, to answer Research question 1: "Can a straightforward game-theoretical extension of a predator-prey Lotka-Volterra model capture summer-season interactions between the predatory mites (Acari: Phytoseiidae) and the fruit-tree red spider mites (Acari: Tetranychidae) with a sufficient level of realism?"

In the game we designed, the time-dependent decisions for the predator and the prey represent the proportion of their populations being active, as opposed to those who are in diapause, which is a state of physiological rest. Here, the fitness functions are different from those known from classical evolutionary game theory: For the prey, its integral fitness 
is a function of the number of individuals entering the diapause during the entire summer season, while for the predator, its fitness depends only on the number of individuals at the end of the summer season, independently of which strategies they have played. We found the time-varying strategies maximizing the predator and the prey fitness via combination of analytical methods (Hamilton-Jacobi-Bellman approach and method of switching surfaces) and accurate constrained-optimization techniques for the discretized version of the problem. As the optimal strategies that we found do not match laboratory and field observations, we answer our research question 1 negatively.

However, if we go beyond this straightforward extension and include internal properties of the individuals involved, as it is done in Staňková et al. (2013a), we can find optimal strategies that match the observations very well. Arguable, such a model is more complex as it involves two more dimensions, however it is still tractable. Another option would be to model predator and prey individuals that are active or in diapause via separate differential equations. Also here the complexity of the model would be increased, however we may get model predictions closer to reality.

In Chapter 2 we did not focus on a spatial version of the predator-prey game, because we considered the nonspatial model sufficient for capturing the critical elements of the interaction at hand. This is partially due to the fact that the laboratory and field observations were carried out in a relatively homogeneous environment where the predatory mites and the fruit-tree red spider mites are well mixed with each other when active. If the field becomes heterogeneous and/or the interacting parties are not well mixed, a spatial model may be needed. Another lesson that we learned when finding optimal strategies for our gametheoretical model, was that even relatively simple models may be analytically intractable and, in such situations, we may do better with numerical computations and/or agent-based simulations.

To understand better what spatial model is more suitable for a specific spatial problem, in Chapter 3 we carried out a study comparing eco-evolutionary (population and frequency) dynamics of models with the same assumptions except of their game field. This chapter was motivated by the observation that there is much more effort invested into researching the difference between eco-evolutionary predictions of very specific spatial and very specific nonspatial models than into understanding how a specific spatial structure influences the prediction of spatial models (Ohtsuki et al., 2007; Roca et al., 2009). We believe that full understanding of the effect of a selected spatial structure on the game dynamics is a key to understand which spatial models are more suited for certain biological problems than others.

The main question that we wanted to answer was Research question 2: "To what extent do the eco-evolutionary dynamics of the continuous-space model, discrete-space model, and replicator dynamics differ from each other and why?"

Our answer to the first part of the question is very clear: The spatial models produce very different eco-evolutionary dynamics, which are still quite different from those of the nonspatial model. When analyzing various scenarios with different assumptions on individual reproduction, dispersal, lifespan and interaction scales, the two types of spatial models generated strikingly different population sizes especially when the space was saturated. We hy- 
pothesized that this difference is caused by the so-called dispersal conflict in a discrete-space model, which occurs when more individuals try to disperse into exactly the same position in the discrete space. We observed that the continuous-space model generally allows a high population size at saturation, while the discrete-space model usually allows only a low population size at saturation, even with the same density-dependence limit. This answers the second part of the research question on why their dynamics differ. The different population sizes at saturation, that are caused by a difficulty to find an empty cell in a discrete-space model while the probability to find an empty spot in a continuous two-dimensional space is 1, explain why the eco-evolutionary outcomes of the discrete-space and continuous-space models often differ. Sometimes, interacting types may go extinct in a discrete space, but they survive in a continuous space at saturation. Moreover, in our case studies the discrete-space model is much more volatile in predictions per run when compared to the continuous-space model and, therefore, less robust to the variations per run caused by the stochastic manner of interactions in our model. When it comes to the stability properties of the outcomes, the continuous-space model shows a more stable behavior than the discrete-space one and concerning the stability of the steady points, the continuous-space model is closer to the dynamics of the nonspatial model. However, so far the discrete-space models are much more computationally efficient than the continuous-space models.

The dispersal conflict stood out as the main cause of the difference in the eco-evolutionary dynamics of the discrete-space and continuous-space games and we found the predictions of the continuous-space model to be more reliable.

There may be other reasons why a certain type of spatial model is more appropriate than another. In our continuous-space model, the players occupy no area as opposed to the discrete model. In microbial interactions, which are the main focus of Chapter 4, the cells are assumed to have almost the same fixed size which does not even vary from strain to strain (Kerr et al., 2002). Therefore, a discrete-space game played over a field composed of equal cells seems appropriate to describe the problem. A microbial individual is reproducing clonally into an empty neighboring cell, while this empty cell is made available when the individual residing at this empty cell dies. This reproduction process is very similar to the death-birth process (Nowak, 2006; Ohtsuki and Nowak, 2006) discussed in Chapter 1 and in such a process the dispersal conflict is not expected. Another reason for selecting the discretespace model is that varying the spatial structure throughout the game was not required, because the interactions took place only among adjacent cells. The main aim of Chapter 4 was to answer Research question 3: "When modeling microbial interaction by means of a discrete-space model, what impact do the so-called interaction matrix (determining whether or not a microbial individual of a particular species kills its neighbor by toxin secretion), the neighborhood size and the interaction rules have on the rate of coexistence for different types of players?"

To answer this question, we carried out a number of case studies to analyze the impact of these factors on coexistence. Comparing discrete-space models with square and hexagonal grids, we concluded that the coexistence rate is negatively correlated with the neighborhood size. This seems intuitive as a higher number of neighbors implies that a focal cell may meet 
its predator more likely. Comparing a partially random selection rule (where each individual in the game field was selected in a random sequence) with a random selection rule (where some individuals may be selected while others not at all), we concluded that the choice of selection rule has only a minor impact on the coexistence rate. Also, we often observed a so-called "coexistence by small numbers" (see Figure 4.6): very few individuals of a certain type can survive until the last generation of simulations, by hiding inside islands formed by harmless neighbors, while no other individuals of this type survive.

We think continuous-space models are most appropriate for modeling tumorigenesis discussed in Chapter 5. This is because in reality cancer cells (i) may disperse into any direction from their original positions and (ii) may have a heterogeneous and time-varying morphology even within the same tumor. Therefore, we believe that modeling cancer requires spatially explicit structures that are less rigid than imposed by discrete-space game fields, in order to capture this flexibility of movement and morphology. In our model, we focused on the impact of the fitness matrices and the three radii that influence the eco-evolutionary (population and frequency) dynamics. These radii are (i) the frequency-dependence radius within which cells interact with each other, (ii) the density-dependence radius within which cells experience limits to population growth, and (iii) the dispersal radius which determines the region in which daughter cells are being put in the field.

The goal of Chapter 5 was to answer Research question 4: "What is the impact of the choice of fitness matrix on the observed processes and in how far do these processes depend on the three radii mentioned before?" Our answer to this question can be summarized as follows: (i) when just two cell types coexist in the nonspatial ESS, then the spatial game leads to stable frequencies equivalent to the ESS of the nonspatial game, (ii) with three cell types coexisting in ESS, discrepancies between the spatial and nonspatial dynamics occur. Such discrepancies happen because cell types become more or less clumped in space, leading to non-random assortative interactions between cell types. Case studies with various spatial scales (i.e., represented as parameters) indicated the following: (i) Increasing the frequency-dependence radius leads to a higher clumping of each cell type, (ii) increasing the density-dependence radius leads to the formation of densely packed tumor clusters in space, (iii) increasing the dispersal radius promotes increased mixing of all cell types. To our knowledge, the effects of these spatial scales on eco-evolutionary dynamics have not been explored in cancer models. The fact that our continuous-space game of metastatic castrate-resistant prostate cancer provides outcomes differing from those of the nonspatial game, supports our claim that classical nonspatial models are insufficient for capturing the key elements of tumorigenesis.

\subsection{Extensions and limitations}

Our spatial and nonspatial models were based on simplified assumptions on real-world biological behaviors and as such could be extended and have certain limitations. We will discuss these extensions and limitations below.

Chapter 2 - Our models can be extended into several directions. First, the introduced 
nonspatial game-theoretical models could be extended into spatial models to describe the predator-prey interactions taking place between individuals that are not well mixed, for example by including diffusion terms into our system of equations. Second, we still need to analyze for what parameter domains the optimal strategies of the predator and prey populations are evolutionary stable. Ultimately, the model with energy dynamics could be also describing a long-term dynamics over multiple seasons. Alternatively, populations of the active and diapaused mites could be modeled as separate state variables, or we could adopt an agent-based approach to model behavior of individual mites. Comparing the predictions of these alternative models with the ones developed in this thesis may lead to a more thorough understanding of the mechanisms influencing the optimal behaviors of the predatory mites and the fruit-tree red spider mites.

Chapter 3 - For the comparison between the two types of spatial dynamics, in this chapter we studied three specific cases of the generalized Rock-Scissors-Paper game. Moreover, we confined ourselves to three different numerical settings for this specific matrix game and we did not go into very complex extensions of our spatial models (with stochastic death/birth process, individual stochastic/deterministic movement, density-dependent payoffs, only portion of individuals interacting, etc). Compared to ESS, a more advanced concept of evolutionary stability seems to be needed to describe stability properties for the spatial models of finite populations more appropriately.

Chapter 4 - There are several possible directions for future research. First, our models assumed that all individual population members remained at fixed positions in space, while in reality the individuals move. We therefore wish to explore the effects of different mobility rules on coexistence and relate our models to other studies on this aspect (Reichenbach et al., 2007). Second, we focused on only two neighborhood sizes and this may be perceived as an insufficient support for our claims. However, considering the stochasticity of the models, theoretical proof of our claim may be impossible to obtain as one would need an accurate population dynamics approximation of the model with a neighborhood of size $n$ in order to be able to use mathematical induction with respect to the neighborhood size to prove our claim. Therefore, a less elegant but certainly feasible option is to carry out case studies with many different neighborhood sizes to confirm our findings. Third, while we observed that a varying neighborhood size may influence the predictions of the spatial models, we would like to compare our predictions to those of the continuous-space model of the same interactions, as in such a continuous-space model, the variance of every individual's number of neighbors may be high. Fourth, it would be interesting to examine what happens if the rates at which one type is killing another type were to be taken into account. Finally, an interesting direction would be to study partial coexistence of types instead of coexistence of all types, as explored in this thesis.

Chapter 5 - There are several future directions. First, tumor development in the transient phase needs to be further investigated, as the main part of this chapter focused on the saturated tumor. Treatment should start at an early stage and this makes studying spatial dynamics in the transient phase important, although sometimes, diagnosis or therapy may not occur until a saturated equilibrium has occurred where a given tumor has reached 
a large size and has become less treatable. Second, the scenario in which rings of dead cells are formed, remains to be further examined, as tumors in such a scenario resemble real tumors for many cancer types. Third, the spatial model can be expanded to include blood vasculature to determine tumor growth and heterogeneity, especially when we want to test various regimes of cancer treatment. This in fact is a topic of our current research efforts, which we believe will make the models more realistic. Also, for some types of cancer a 3dimensional model may represent the tumorigenesis more realistically than a 2-dimensional model (Chitcholtan et al., 2012). It would be interesting to see in how far an extension of our model from 2-dimensional space into 3-dimensional space still provides comparable results.

Moreover, in our spatial model the proliferation probabilities present in the fitness matrices satisfy inequalities representing a tumor after androgen deprivation therapy, while the specific values for these probabilities were selected more or less randomly. The main reason for this is that at the moment it is impossible to measure the proliferation probabilities of cancer cells in vivo. With advancement of the techniques for measuring tumorigenesis we should be able to validate our model with the real data. As our model is spatial, it could be additionally validated using MRI imaging once such imaging becomes more frequent and once we overcome difficulties with imaging of metastases present in the bone, which is very typical for metastatic castrate-resistant prostate cancer. Finally, our models can help with designing the so-called evolutionary enlightened cancer treatment. This treatment focuses on controlling the evolution of resistant cancer phenotypes as opposed to a more traditional "treat to kill" regimen, which tries to kill all cancer cells and usually fails due to evolution of resistance in cancer cells.

\subsection{Research avenues more far ahead}

This thesis opens up several new promising research avenues. It allows for studying some spatio-temporal characteristics of human population. For example, our spatial models can be adopted for studying rural-urban migration and for modeling and predicting (human) population growth. These proposed research avenues are explained in more detail below.

Studying rural-urban migration via spatial evolutionary games - In many countries, rates of rural-urban migration (i.e. movement of people from rural areas to urban areas within a country, often the metropolitan cities of a country; also known as urbanization) are increasing (Zhang and Shunfeng, 2003; Lucas and Robert, 2004) and more than half of the world's population now lives in cities (World Health Organization, 2016). While there are many causes for rural-urban migration, the economic reasons outweigh the non-economic ones (Lucas and Robert, 2004; Williamson, 1988). In general, increase in rural-urban migration may lead to problems such as transport congestion, lack of sufficient housing, environmental degradation, over-crowdedness and higher chances for outbreak of disease/epidemics in the metropolitan areas. Lucas and Robert (2004) reports that for example in Mexico or Philippines high portions of immigrants to the metropolitan cities are worse off than they were in the rural areas they came from, as metropolitan cities display severe inequalities in housing provision, healthcare and employment. Some people want to escape these problems 
by moving away from the cities. This process is referred to as counter-urbanization (Nefedova et al., 2016). While the data regarding rural-urban migration accumulates and some researchers have tried to analyze and predict the rural-urban migration using mathematical or simulation models (Liu et al., 2005; Li et al., 2012), just few of these models are spatially explicit (O'sullivan and Torrens, 2001; Dabbaghian et al., 2010). In these spatial models, cellular automata (CA) have been used in studying rural-urban migration. These models considered local interactions between cells representing households and included (positive or negative) local events (such as job opportunities, job loss, crime, marriages, etc.) to match census data, while eco-evolutionary equilibria of these models were not discussed. To our best knowledge, no game-theoretical model which focuses on long-term effects of spatio-temporal individual choices on the rural-urban migration exists. To bridge this gap, we can adjust the spatially explicit game-theoretical models introduced in this thesis to examine this process, with fitness payoffs varying in both space and time (as environmental conditions, educational opportunities, unemployment do), and player types relating to different migration decisions.

Modeling (human) population growth - Studying (human) population growth has always attracted much interest (Meyer and II, 1992; Ehrlich and Holdren, 1971; Gerland et al., 2014), as only good understanding of the population growth can lead to useful decisions regarding some population-related issues, such as population migration management policy, land use planning, educational resources configuration. The rapid population growth is without doubt one of the critical societal issues nowadays (Gerland et al., 2014). The world's population has doubled in the past 50 years, and is still increasing at a rapid pace (Bongaarts, 2009). This rapid population growth, particularly observed in developing countries, is not only a demographic problem, but also influences social, economic and environment development. According to Gerland et al. (2014); Lam and Duryea (1999); Malhotra and Schüler (2005); Lan and Kuang (2016), there are multiple determinants that can slow down population growth. For example, women's increasing educational attainment stands out as contributing to population decline in most developing countries. Not only because women's improved education exposes them to modern values and ideas that emphasize individualism, but also because women's economic independence enables them to decide for themselves on the number of children they desire. Another example may be the Chinese state birth control policy, as the country's fertility decline (since the 1970's) is directly related to such a birth control policy, but is perceived as a very controversial (and often unethical) measure (Coale, 1984). Except of these determinants, there are other factors that might impact population growth, such as immigration or gender-bias policies, or individual behaviors regarding selecting jobs or partners (Bongaarts, 2009). These individual behaviors can be interpreted as interactions in the "game of living". Just as how we specified the rules of generalized Rock-Scissors-Paper games (Chapter 3), microbial interactions (Chapter 4) and tumorigenesis (Chapter 5), the rules of a game of living regarding individuals' status (such as an employment or marriage status, fertility, retirement age, or expected life length) can be incorporated into the models that we have developed. For example, age is a factor that affects someone's education, marital status, giving birth to a child, death, etc. The education level of an individual can be estimated based on simple rules and probabilities, 
e.g., a child starts attending a primary school at the age of 6 or 7 years with a certain probability; he or she will spend 6 years in primary school with a certain probability, after that he or she goes to a junior high school with a certain probability, etc. Fertility of both men and women is age-dependent, education-dependent and policy-dependent. By varying these factors in our models, we can examine the sensitivity of population growth with respect to these determinants. Understanding their impact on population growth and decline is very meaningful for finding proper solutions to the rapid population growth. 


\section{Summary}

This thesis focuses on modeling and understanding biological interactions through spatial and nonspatial evolutionary game theory (EGT). In these interactions, one's well-being often depends on how the others are doing, making EGT an appropriate tool for understanding biological problems.

In this thesis we apply three different game-theoretical (spatial and nonspatial) models for studying three different kinds of biological interactions, respectively: a nonspatial model for the predator-prey interactions among mites (Chapter 2), a discrete-space model for competition among microbial strains (Chapter 4) and a continuous-space model for tumor growth involving three distinct types of cancer cells (Chapter 5). In Chapter 3 we investigate properties of spatial structures which may influence the eco-evolutionary (population and frequency) dynamics dramatically. Each of the Chapters 2 to 5 deals with a specific research question, and how we apply spatial/nonspatial game-theoretical models for addressing these research questions shapes this thesis.

In Chapter 2 we implement a nonspatial game for modeling the summer-season interactions between the predatory mites (Acari: Phytoseiidae) and the fruit-tree red spider mites (Acari: Tetranychidae). Based on biological observations, we design a nonspatial game which expands the classical Lotka-Volterra two dimensional system of ordinary differential equations. In this nonspatial game, player decisions ("joining" or "opting out") are time-varying, fitness of the prey is an integral function with respect to the number of individuals playing "opting out" during the entire summer season, and fitness of the predator is only dependent on the number of individuals (regardless of which strategy is played) at the end of the summer season. Combining analytical and numerical methods, we seek time-varying optimal decisions for the predator and prey which maximize their fitnesses. Our results do not match empirical observations. Our nonspatial model may be oversimplified and overlooking internal properties of the predator and the prey. A more complex game-theoretical model, proposed in Staňková et al. (2013a), suggests that one should include energy levels of predator and prey individuals to reflect the studied predator-prey interactions more realistically. 
Chapter 3 focuses on a comparison of dynamics of discrete-space, continuous-space and nonspatial games. We propose discrete-space and continuous-space dynamics as spatial extensions of the (nonspatial) replicator dynamics. We use agent-based simulations to track the eco-evolutionary dynamics of the spatial models. We observe that the two types of spatial models generate strikingly different eco-evolutionary dynamics, which are also different from those of the nonspatial ones. We hypothesize that this difference between discrete-space and continuous-space games is caused by the so-called dispersal conflict (i.e., when more individuals try to disperse simultaneously into exactly the same position in the discrete space). When compared to the discrete-space model, we find the continuous-space model to be more robust to variations per run, due to the stochastic nature of game rules, but much less computationally efficient. To our best knowledge, this chapter is the first work that devotes itself to understanding how different types of specific spatial structures influence the predictions of spatial models.

Chapter 4 demonstrates an application of discrete-space games in studying scenarios that lead to coexistence of microbial species. In this chapter we use discrete-space models to mimic scenarios which examine the impact of the interaction matrix, of a varying fixed neighborhood size and of different manners how the microbes are selected for interaction. Here the interaction matrix is a binary matrix indicating whether cells of a specific type can or cannot kill cells of a certain other type. Through case studies we observe that a more dense interaction matrix leads to a lower coexistence rate. We also observe that a higher coexistence rate is achieved with a relatively low neighborhood size when square and hexagonal grids are compared. The exact manner of selecting a focal cell seems to have only a minor influence on the coexistence.

Chapter 5 demonstrates an application of continuous-space games in modeling tumorigenesis of metastatic castrate-resistant prostate cancer involving three distinct types of cancer cells. It is possible to achieve different levels of local saturation in a continuous-space model and this feature makes the continuous-space model more appropriate for modeling cancer, whose cells can grow throughout space and form very variable local densities. Based on the state of the art cancer biology for these types, 22 potential fitness matrices are examined. Further, we study the impact of three radii (i.e., frequency-dependence, density-dependence and dispersal radii) on the eco-evolutionary dynamics of a tumor. We use agent-based simulations to track the eco-evolutionary dynamics. Some of the predictions of our spatial models nicely match observations on the development of real tumors. Moreover, we see that the spatial dynamics are very different from the nonspatial ones, unless we make one of the three radii very large. This supports our claim that nonspatial models are insufficient for modeling tumorigenesis.

Finally in Chapter 6 we conclude the results of this thesis through a global perspective and pose several directions for future research. 


\section{Addendum: Valorization}

Valorization is "the process of value creation from knowledge, by making it applicable and available for economic or societal utilization, and by translating it in the form of new business, products, services, or processes". Examples of valorization include publications in (academic or non-academic) journals, software programs, patents, ready-to-use models and systems, suggestions to society, organizations or companies, etc. In this addendum we describe the valorization opportunities that result from this thesis. Through the following three perspectives we demonstrate how this thesis can be of value for societal development.

Social relevance of the research Applying three different game-theoretical models, we addressed three specific biological problems that are of a societal and economic relevance.

The model of the predator-prey game (Chapter 2) relates to the use of the predatory mites (Acari: Phytoseiidae) for biological pest control of the fruit-tree red spider mites (Acari: Tetranychidae) which feed on and damage apple trees. Traditionally chemicals are used to control pest directly (Uboh et al., 2011), but it is shown that these chemicals have many side effects. For example, chemicals that reside in the agricultural ecosystems not only contaminate the environment, but may also cause human diseases like cancer (Mostafalou and Abdollahi, 2013). Instead, using biological pest control (in agriculture) meets the societal demand for healthy environmental conditions. Moreover, understanding the predator-prey interactions (for problems at hand) helps to develop effective biological pest-control techniques, which may also bring economic benefits. For example, better understanding of the interactions between the predatory mites (Acari: Phytoseiidae) and the fruit-tree red spider mites (Acari: Tetranychidae) may help farmers to use the predatory mites as a biological pest management. Alternatively, one can intervene in the behavior of the prey/pest by artificially changing the factors like temperature or light, such that the apple trees remain undamaged.

The model describing interaction/competition among microbial species (Chapter 4) addresses mechanisms of microbial diversity. There is no doubt that microbial diversity proves 
to be very important to humans. Besides, this model also includes one type of the quorum sensing mechanism that relates to virulence, i.e., the ability of a pathogen to infect a host. The understanding of how bacteria coordinate and turn on their group behavior (as attacking the host) can be very useful for developing new techniques that prevent pathogenicity (Jayaraman and Wood, 2008). Antibiotics are what we use now to kill bacteria. However, these are becoming less effective (World Health Organization, 2017) because killing bacteria with (traditional) antibiotics selects for resistant mutants. Novel treatment strategies may focus on interfering the bacterial quorum sensing mechanisms such that the bacteria become less virulent. Such a treatment strategy may be more effective in controlling infectious diseases and would be beneficial to the society. Our model mimics a simple case of quorum sensing, but more complex mechanisms of quorum sensing can be studied as well.

The chapter on tumorigenesis (Chapter 5) addresses the eco-evolutionary dynamics of metastatic castrate-resistant prostate cancer. Understanding these dynamics will be very helpful in designing effective cancer treatment. The standard metastatic cancer therapy is to kill as many cancer cells as possible by applying the maximum tolerable dose (MTD) of drugs. Initially, this approach is often successful in reducing the tumor burden, but it inevitably fails due to evolution of resistant cancer phenotypes. The MTD focuses only on decreasing the tumor mass but it ignores the evolution of the tumor composition. However, an effective treatment should also take into account how the cancer responds both in terms of tumor mass and its composition. Moreover, such an effective treatment requires lower doses of drugs (than the standard treatment does) and as such treatment becomes less expensive. This more adaptive treatment is expected to lengthen patients' lives and therefore has a high potential of being beneficial for society.

Target groups of the research results The results of this research, as summarized in Chapter 6, can be of interest to various target groups, but mainly to oncologists, cancer biologists, microbiologists and those working in pest control. For game theorists the models developed in this thesis are interesting from the application perspective.

Products that the research holds promise to The main products that resulted from this thesis are software toolboxes for modeling biological interactions which can be applied to a wide range of domains. We expect that the availability of more and more data on for example pest control or tumorigenesis will allow us to tune and validate our models as the codes in the toolboxes can be flexibly adjusted. So far, these toolboxes are still in a testing phase but we expect that in due time these can be utilized commercially for making accurate predictions. 


\section{Bibliography}

Abrudan, M., You, L., Staňková, K., and Thuijsman, F. (2016). A game theoretical approach to microbial coexistence. In Thuijsman, F. and Wagener, F., editors, Advances in Dynamic and Evolutionary Games. Annals of the International Society of Dynamic Games, volume 14, chapter 13, pages 267-282. Springer. 47, 112

Adami, C., Schossau, J., and Hintze, A. (2016). Evolutionary game theory using agent-based methods. Physics of Life Reviews, 19(1):1-26. 48, 49

Akin, E. (2010). The General Topology of Dynamical Systems (Graduate Studies in Mathematics). American Mathematical Society. 11

Altrock, P. M., Liu, L. L., and Michor, F. (2015). The mathematics of cancer: integrating quantitative models. Nature Reviews Cancer, 15(12):730-745. 89

Altrock, P. M. and Traulsen, A. (2009). Deterministic evolutionary game dynamics in finite populations. Physical Review E, 80(1). paper nr. 011909. 89

Arrowsmith, D. and Place, C. (1992). Dynamical Systems: Differential Equations, Maps, and Chaotic Behaviour. Mathematics Series. CRC Press. 11

Ashforth, B. E. and Mael, F. (1989). Social identity theory and the organization. Academy of Management Review, 14(1):20-39. 16

Avelino, P., Bazeia, D., Losano, L., Menezes, J., and de Oliveira, B. (2014). Interfaces with internal structures in generalized rock-paper-scissors models. Physical Review E, 89(4). paper nr. 042710. 16, 18, 47, 48, 52

Axelrod, R., Ford, G. R., Riolo, R. L., and Cohen, M. D. (2002). Beyond geography: cooperation with persistent links in the absence of clustered neighborhoods. Personality and Social Psychology Review, 6(4):341-346. 16 
Backstrom, L., Huttenlocher, D., Kleinberg, J., and Lan, X. (2006). Group formation in large social networks: membership, growth, and evolution. In Ungar, L., Craven, M., Gunopulos, D., and Eliassi-Rad, T., editors, Proceedings of the 12th ACM SIGKDD International Conference on Knowledge Discovery and Data Mining, pages 44-54. ACM. 16

Basanta, D., Hatzikirou, H., and Deutsch, A. (2008). Studying the emergence of invasiveness in tumours using game theory. The European Physical Journal B, 63(3):393-397. 90

Basanta, D., Scott, J. G., Fishman, M. N., Ayala, G., Hayward, S. W., and Anderson, A. R. (2012). Investigating prostate cancer tumour-stroma interactions: clinical and biological insights from an evolutionary game. British Journal of Cancer, 106(1):174-181. 90, 91

Başar, T. and Olsder, G. J. (1995). Dynamic noncooperative games. Academic Press. 29

Bascompte, J., Jordano, P., and Olesen, J. M. (2006). Asymmetric coevolutionary networks facilitate biodiversity maintenance. Science, 312(5772):431-433. 73

Bedard, P. L., Hansen, A. R., Ratain, M. J., and Siu, L. L. (2013). Tumour heterogeneity in the clinic. Nature, 501(7467):355-364. 89

Beerenwinkel, N., Schwarz, R. F., Gerstung, M., and Markowetz, F. (2015). Cancer evolution: mathematical models and computational inference. Systematic Biology, 64(1):e1-e25. 90

Bellman, R. (1957). Dynamic Programming. Princeton University Press. 29, 37, 41

Bishop, D. and Cannings, C. (1978). A generalized war of attrition. Journal of Theoretical Biology, 70(1):85-124. 13

Bloembergen, D. (2015). Multiagent Learning Dynamics. PhD thesis, Maastricht University, The Netherlands. 10

Bomze, I. M. (1983). Lotka-Volterra equation and replicator dynamics: a two-dimensional classification. Biological Cybernetics, 48(3):201-211. 13, 15, 50, 92

Bongaarts, J. (2009). Human population growth and the demographic transition. Philosophical Transactions of the Royal Society B: Biological Sciences, 364(1532):2985-2990. 121

Boyd, R. and Richerson, P. J. (2002). Group beneficial norms can spread rapidly in a structured population. Journal of Theoretical Biology, 215(3):287-296. 15

Brauchli, K., Killingback, T., and Doebeli, M. (1999). Evolution of cooperation in spatially structured populations. Journal of Theoretical Biology, 200(4):405-417. 17, 47, 48

Bronshtein, I., Semendyayev, K., Musiol, G., and Mühlig, H. (2015). Handbook of Mathematics, 16th Edition. Springer-Verlag Berlin Heidelberg. 11 
Cai, C., Chen, S., Ng, P., Bubley, G. J., Nelson, P. S., Mostaghel, E. A., Marck, B., Matsumoto, A. M., Simon, N. I., and Wang, H. (2011). Intratumoral de novo steroid synthesis activates androgen receptor in castration-resistant prostate cancer and is upregulated by treatment with CYP17A1 inhibitors. Cancer Research, 71(20):6503-6513. 91

Cardillo, A., Meloni, S., Gómez-Gardeñes, J., and Moreno, Y. (2012). Velocity-enhanced cooperation of moving agents playing public goods games. Physical Review E, 85(6). paper nr. 067101. 16, 18, 47

Chao, L. and Levin, B. R. (1981). Structured habitats and the evolution of anticompetitor toxins in bacteria. Proceedings of the National Academy of Sciences, 78(10):6324-6328. 73

Chitcholtan, K., Sykes, P. H., and Evans, J. J. (2012). The resistance of intracellular mediators to doxorubicin and cisplatin are distinct in 3D and 2D endometrial cancer. Journal of Translational Medicine, 10(1):38-54. 120

Coale, A. (1984). Rapid Population Change in China, 1952-1982. National Academy Press. 121

Cohen, M. D., Riolo, R. L., and Axelrod, R. (2001). The role of social structure in the maintenance of cooperative regimes. Rationality and Society, 13(1):5-32. 17

Consortium, T. H. M. P. (2012). Structure, function and diversity of the healthy human microbiome. Nature, 486(7402):207-214. 73

Cooper, R. W., DeJong, D. V., Forsythe, R., and Ross, T. W. (1990). Selection criteria in coordination games: Some experimental results. The American Economic Review, 80(1):218-233. 4

Cressman, R. (2003). Evolutionary Dynamics and Extensive Form Games. MIT Press. 13

Czárán, T. L., Hoekstra, R. F., and Pagie, L. (2002). Chemical warfare between microbes promotes biodiversity. Proceedings of the National Academy of Sciences, 99(2):786-790. 52

Dabbaghian, V., Jackson, P., Spicer, V., and Wuschke, K. (2010). A cellular automata model on residential migration in response to neighborhood social dynamics. Mathematical and Computer Modelling, 52(9):1752-1762. 121

Danks, H. (1987). Insect Dormancy: An Ecological Perspective. Biological Survey of Canada. 25,26

De Saizieu, A., Gardès, C., Flint, N., Wagner, C., Kamber, M., Mitchell, T. J., Keck, W., Amrein, K. E., and Lange, R. (2000). Microarray-based identification of a novelstreptococcus pneumoniae regulon controlled by an autoinduced peptide. Journal of Bacteriology, 182(17):4696-4703. 77 
Delmont, T. O., Robe, P., Cecillon, S., Clark, I. M., Constancias, F., Simonet, P., Hirsch, P. R., and Vogel, T. M. (2011). Accessing the soil metagenome for studies of microbial diversity. Applied and Environmental Microbiology, 77(4):1315-1324. 73

Dingli, D., Chalub, F., Santos, F., Van Segbroeck, S., and Pacheco, J. (2009). Cancer phenotype as the outcome of an evolutionary game between normal and malignant cells. British Journal of Cancer, 101(7):1130-1136. 9, 90

Dunne, J. A., Williams, R. J., and Martinez, N. D. (2002). Network structure and biodiversity loss in food webs: robustness increases with connectance. Ecology Letters, $5(4): 558-567.73$

Durrett, R. and Levin, S. (1997). Allelopathy in spatially distributed populations. Journal of Theoretical Biology, 185(2):165-171. 47, 48

Durrett, R. and Levin, S. (1998). Spatial aspects of interspecific competition. Theoretical Population Biology, 53(1):30-43. 47, 52

Dykhuizen, D. E. (1998). Santa rosalia revisited: why are there so many species of bacteria? Antonie van Leeuwenhoek, 73(1):25-33. 73

Egeblad, M., Nakasone, E. S., and Werb, Z. (2010). Tumors as organs: complex tissues that interface with the entire organism. Developmental Cell, 18(6):884-901. 89

Ehrlich, P. R. and Holdren, J. P. (1971). Impact of population growth. Science, 171(3977):1212-1217. 121

Engelberg-Kulka, H., Amitai, S., Kolodkin-Gal, I., and Hazan, R. (2006). Bacterial programmed cell death and multicellular behavior in bacteria. PLoS Genetics, 2(10):15181526. 76

Ferriere, R. and Michod, R. E. (1995). Invading wave of cooperation in a spatial iterated prisoner's dilemma. Proceedings of the Royal Society of London B: Biological Sciences, 259(1354):77-83. 15

Fitzgerald, J. and Solomon, M. (1991). Diapause induction and duration in the phytoseiid mite Typhlodromus pyri. Experimental \& Applied Acarology, 12(2):135-145. 25

Fizazi, K., Bosserman, L., Gao, G., Skacel, T., and Markus, R. (2013). Denosumab treatment of prostate cancer with bone metastases and increased urine n-telopeptide levels after therapy with intravenous bisphosphonates: results of a randomized phase II trial. The Journal of Urology, 189(1):509-516. 91

Friedl, P. and Wolf, K. (2003). Tumour-cell invasion and migration: diversity and escape mechanisms. Nature Reviews Cancer, 3(5):362-374. 111

Funkhouser, L. J. and Bordenstein, S. R. (2013). Mom knows best: the universality of maternal microbial transmission. PLoS Biology, 11(8). paper nr. e1001631. 73 
Gallaher, J. and Anderson, A. R. (2013). Evolution of intratumoral phenotypic heterogeneity: the role of trait inheritance. Interface Focus, 3(4). paper nr. 20130016. 18, 47, 89, 110

Gallaher, J. A., Enriquez-Navas, P. M., Luddy, K. A., Gatenby, R. A., and Anderson, A. R. (2017). Adaptive therapy for heterogeneous cancer: Exploiting space and trade-offs in drug scheduling. bioRxiv. paper nr. 128959. 18, 47

Gatenby, R. A., Brown, J., and Vincent, T. (2009a). Lessons from applied ecology: cancer control using an evolutionary double bind. Cancer Research, 69(19):7499-7502. 113

Gatenby, R. A., Cunningham, J. J., and Brown, J. S. (2014). Evolutionary triage governs fitness in driver and passenger mutations and suggests targeting never mutations. Nature Communications, 5. paper nr. 5499. 89

Gatenby, R. A., Silva, A. S., Gillies, R., and Frieden, B. R. (2009b). Adaptive therapy. Cancer Research, 69(11):4894-4903. 113

Gerland, P., Raftery, A. E., Ševčíková, H., Li, N., Gu, D., Spoorenberg, T., Alkema, L., Fosdick, B. K., Chunn, J., and Lalic, N. (2014). World population stabilization unlikely this century. Science, 346(6206):234-237. 121

Gerlee, P. and Anderson, A. R. (2007). An evolutionary hybrid cellular automaton model of solid tumour growth. Journal of Theoretical Biology, 246(4):583-603. 112

Godfrey Smith, P. (2009). Darwinian Populations and Natural Selection. Oxford University Press. 7, 48

Gordon, S. (2003). Alternative activation of macrophages. Nature Reviews Immunology, 3(1):23-35. 110

Grim, P. (1996). Spatialization and greater generosity in the stochastic prisoner's dilemma. BioSystems, 37(1):3-17. 17

Hamilton, M. P., Rajapakshe, K. I., Bader, D. A., Cerne, J. Z., Smith, E. A., Coarfa, C., Hartig, S. M., and McGuire, S. E. (2016). The landscape of microRNA targeting in prostate cancer defined by AGO-PAR-CLIP. Neoplasia, 18(6):356-370. 91

Hauert, C. (2010). Replicator dynamics of reward \& reputation in public goods games. Journal of Theoretical Biology, 267(1):22-28. 15

Hauert, C., De Monte, S., Hofbauer, J., and Sigmund, K. (2002). Replicator dynamics for optional public good games. Journal of Theoretical Biology, 218(2):187-194. 15

Hauert, C. and Doebeli, M. (2004). Spatial structure often inhibits the evolution of cooperation in the snowdrift game. Nature, 428(6983):643-646. 17, 90

Helbing, D. (2009). Pattern formation, social forces, and diffusion instability in games with success-driven motion. The European Physical Journal B, 67(3):345-356. 15 
Helle, W. and Sabelis, M. W. (1985). Spider Mites: their Biology, Natural Enemies and Control. Elsevier Amsterdam. 25, 26

Hofbauer, J. (1981). On the occurrence of limit cycles in the Volterra-Lotka equation. Nonlinear Analysis: Theory, Methods \& Applications, 5(9):1003-1007. 14, 143, 144

Hofbauer, J. and Sigmund, K. (1998). Evolutionary Games and Population Dynamics. Cambridge University Press. 13, 15, 48, 49, 50, 52, 53, 90, 92, 153

Hofbauer, J. and Sigmund, K. (2003). Evolutionary game dynamics. Bulletin of the American Mathematical Society, 40(4):479-519. 13

Hofbauer, J., Sorin, S., and Viossat, Y. (2009). Time average replicator and best-reply dynamics. Mathematics of Operations Research, 34(2):263-269. 15

Huang, Y. and Wu, Z. (2012). Game dynamic model for yeast development. Bulletin of Mathematical Biology, 74(7):1469-1484. 49

Huberman, B. A. and Glance, N. S. (1993). Evolutionary games and computer simulations. Proceedings of the National Academy of Sciences, 90(16):7716-7718. 18

Hunt, K. M., Foster, J. A., Forney, L. J., Schütte, U. M., Beck, D. L., Abdo, Z., Fox, L. K., Williams, J. E., McGuire, M. K., and McGuire, M. A. (2011). Characterization of the diversity and temporal stability of bacterial communities in human milk. PLoS One, 6(6). paper nr. e21313. 73

Hussain, M., Tangen, C. M., Berry, D. L., Higano, C. S., Crawford, E. D., Liu, G., Wilding, G., Prescott, S., Kanaga Sundaram, S., and Small, E. J. (2013). Intermittent versus continuous androgen deprivation in prostate cancer. New England Journal of Medicine, 368(14):1314-1325. 91

Hutson, V. and Vickers, G. (1995). The spatial struggle of tit-for-tat and defect. Philosophical Transactions of the Royal Society of London B: Biological Sciences, 348(1326):393-404. 16

Jayaraman, A. and Wood, T. K. (2008). Bacterial quorum sensing: signals, circuits, and implications for biofilms and disease. Annual Review of Biomedical Engineering, 10(1):145167. 126

Kerr, B., Riley, M. A., Feldman, M. W., and Bohannan, B. J. (2002). Local dispersal promotes biodiversity in a real-life game of rock-paper-scissors. Nature, 418(6894):171174. 18, 47, 48, 52, 69, 73, 75, 90, 117

Killingback, T. and Doebeli, M. (1996). Spatial evolutionary game theory: Hawks and doves revisited. Proceedings of the Royal Society of London B: Biological Sciences, 263(1374):1135-1144. 17, 47 
Kinkel, L. L., Schlatter, D. C., Xiao, K., and Baines, A. D. (2014). Sympatric inhibition and niche differentiation suggest alternative coevolutionary trajectories among streptomycetes. The ISME Journal, 8(2). paper nr. 249. 74

Kirkup, B. C. and Riley, M. A. (2004). Antibiotic-mediated antagonism leads to a bacterial game of rock-paper-scissors in vivo. Nature, 428(6981):412-414. 52

Kline, M. (1990). Mathematical Thought from Ancient to Modern times: Volume 3. Oxford University Press. 12

Kozusko, F. and Bajzer, Ž. (2003). Combining Gompertzian growth and cell population dynamics. Mathematical Biosciences, 185(2):153-167. 89

Kroon, A., Veenendaal, R. L., Bruin, J., Egas, M., and Sabelis, M. W. (2004). Predation risk affects diapause induction in the spider mite Tertanychus urticae. Experimental and Applied Acarology, 34(3):307-314. 25, 26

Kroon, A., Veenendaal, R. L., Bruin, J., Egas, M., and Sabelis, M. W. (2008). "Sleeping with the enemy"- predator-induced diapause in a mite. Naturwissenschaften, 95(12):1195-1198. $25,26,39$

Kroon, A., Veenendaal, R. L., Egas, M., Bruin, J., and Sabelis, M. W. (2005). Diapause incidence in the two-spotted spider mite increases due to predator presence, not due to selective predation. Experimental and Applied Acarology, 35(1):73-81. 25, 26

Lam, D. and Duryea, S. (1999). Effects of schooling on fertility, labor supply, and investments in children, with evidence from brazil. Journal of Human Resources, 34(1):160-192. 121

Lan, M. and Kuang, Y. (2016). The impact of women's education, workforce experience, and the one child policy on fertility in China: a census study in Guangdong, China. SpringerPlus, 5(1):1708-1720. 121

Lee, S. J., Lee, D. J., and Oh, H. S. (2005). Technological forecasting at the korean stock market: a dynamic competition analysis using Lotka-Volterra model. Technological Forecasting and Social Change, 72(8):1044-1057. 15

Ley, R. E., Hamady, M., Lozupone, C., Turnbaugh, P. J., Ramey, R. R., Bircher, J. S., Schlegel, M. L., Tucker, T. A., Schrenzel, M. D., and Knight, R. (2008). Evolution of mammals and their gut microbes. Science, 320(5883):1647-1651. 73

Li, Y., Li, Y., Zhou, Y., Shi, Y., and Zhu, X. (2012). Investigation of a coupling model of coordination between urbanization and the environment. Journal of Environmental Management, 98:127-133. 121

Lindgren, K. and Nordahl, M. G. (1994). Evolutionary dynamics of spatial games. Physica D: Nonlinear Phenomena, 75(1):292-309. 16 
Lion, S. and Baalen, M. (2008). Self-structuring in spatial evolutionary ecology. Ecology Letters, 11(3):277-295. 47

Liu, B., Chen, L., , and Zhang, Y. (2005). The dynamics of a prey-dependent consumption model concerning impulsive control strategy. Applied Mathematics and Computation, 169(1):305-320. 121

Lotka, A. J. (1926). Elements of physical biology. Science Progress in the Twentieth Century (1919-1933), 21(82):341-343. 14, 25

Lucas, J. and Robert, E. (2004). Life earnings and rural-urban migration. Journal of Polictical Economy, 112(1):S29-S59. 120

Macklin, P. and Edgerton, M. E. (2010). Agent-based Cell Modeling: Application to Breast Cancer. Cambridge University Press. 89

Malcai, O., Biham, O., Richmond, P., and Solomon, S. (2002). Theoretical analysis and simulations of the generalized Lotka-Volterra model. Physical Review E, 66(3). paper nr. 031102. 15

Malhotra, A. and Schüler, S. R. (2005). Women's empowerment as a variable in international development. Measuring Empowerment: Cross-disciplinary Perspectives, 2005:71-88. 121

Mansury, Y., Kimura, M., Lobo, J., and Deisboeck, T. S. (2002). Emerging patterns in tumor systems: simulating the dynamics of multicellular clusters with an agent-based spatial agglomeration model. Journal of Theoretical Biology, 219(3):343-370. 111

Marusyk, A., Almendro, V., and Polyak, K. (2012). Intra-tumour heterogeneity: a looking glass for cancer? Nature Reviews Cancer, 12(5):323-334. 89

Mateo, F., Meca-Cortés, Ó., Celià-Terrassa, T., Fernández, Y., Abasolo, I., Sánchez-Cid, L., Bermudo, R., Sagasta, A., Rodríguez-Carunchio, L., and Pons, M. (2014). SPARC mediates metastatic cooperation between CSC and non-CSC prostate cancer cell subpopulations. Molecular Cancer, 13(1). paper nr. 237. 90

Maynard Smith, J. (1974). The theory of games and the evolution of animal conflicts. Journal of Theoretical Biology, 47(1):209-221. 2, 8

Maynard Smith, J. (1982). Evolution and the Theory of Games. Cambridge University Press. 13

Maynard Smith, J. and Price, G. R. (1973). The logic of animal conflict. Nature, 246(5427):15-18. 6, 7

McGill, B. J., Etienne, R. S., Gray, J. S., Alonso, D., Anderson, M. J., Benecha, H. K., Dornelas, M., Enquist, B. J., Green, J. L., and He, F. (2007). Species abundance distributions: moving beyond single prediction theories to integration within an ecological framework. Ecology Letters, 10(10):995-1015. 84 
McKay, B. D. and Piperno, A. (2014). Practical graph isomorphism II. Journal of Symbolic Computation, 60:94-112. 77

McNamara, J. (2001). Optimality models in behavioral biology. SIAM Review, 43(3):413466. 43

McNamara, J. and Houston, A. I. (1996). State-dependent life histories. Nature, $380(6571): 215-221.43$

Melikyan, A. A. (1994). Necessary optimality conditions for a singular surface in the form of synthesis. Journal of Optimization Theory and Applications, 82(2):203-217. 29, 30

Melikyan, A. A. (1998). Generalized Characteristics of First Order PDEs: Applications in Optimal Control and Differential Games. Birkhäuser. 26, 30, 34, 37

Melikyan, A. A. and Olsder, G. J. (2010). Boundary singularities and characteristics of Hamilton-Jacobi equation. Journal of Dynamical and Control Systems, 16(1):77-99. 30

Merlo, L. M., Pepper, J. W., Reid, B. J., and Maley, C. C. (2006). Cancer as an evolutionary and ecological process. Nature Reviews Cancer, 6(12):924-935. 89

Metz, J., Staňková, K., and Johansson, J. (2016). The adaptive dynamics of life histories: From fitness-returns to selection gradients and pontryagin's maximum principle. Journal of Mathematical Biology, 72(4):1125-1152. 29

Meyer, W. B. and II, B. L. T. (1992). Human population growth and global land-use/cover change. Annual Review of Ecology and Systematics, 23(1):39-61. 121

Miller, M. B. and Bassler, B. L. (2001). Quorum sensing in bacteria. Annual Reviews in Microbiology, 55(1):165-199. 75

Montoya, J. M., Pimm, S. L., and Solé, R. V. (2006). Ecological networks and their fragility. Nature, 442(7100):259-264. 73

Mostafalou, S. and Abdollahi, M. (2013). Pesticides and human chronic diseases: evidences, mechanisms and perspectives. Toxicology and Applied Pharmacology, 268(2):157-177. 125

Mostaghel, E. A., Marck, B. T., Plymate, S. R., Vessella, R. L., Balk, S., Matsumoto, A. M., Nelson, P. S., and Montgomery, R. B. (2011). Resistance to CYP17A1 inhibition with abiraterone in castration-resistant prostate cancer: induction of steroidogenesis and androgen receptor splice variants. Clinical Cancer Research, 17(18):5913-5925. 91

Müller Klieser, W., Schreiber Klais, S., Walenta, S., and Kreuter, M. (2002). Bioactivity of well-defined green tea extracts in multicellular tumor spheroids. International Journal of Oncology, 21(6):1307-1315. 2

Nakamaru, M., Matsuda, H., and Iwasa, Y. (1997). The evolution of cooperation in a lattice-structured population. Journal of Theoretical Biology, 184(1):65-81. 17, 47, 48 
Nash, J. F. (1950). Equilibrium points in n-person games. Proceedings of the National Academy of Sciences, 36(1):48-49. 4, 5, 8

Nathan, C. (2006). Neutrophils and immunity: challenges and opportunities. Nature Reviews Immunology, 6(3):173-182. 110

Nefedova, T., Pokrovskii, N., and Treivish, A. (2016). Urbanization, counterurbanization, and rural-urban communities facing growing horizontal mobility. Sociological Research, 55(3):195-210. 121

Noailly, J., van den Bergh, J. C., and Withagen, C. A. (2009). Local and global interactions in an evolutionary resource game. Computational Economics, 33(2):155-173. 47, 48, 49

Nowak, M. A. (2006). Five rules for the evolution of cooperation. Science, 314(5805):15601563. 49, 90, 117

Nowak, M. A. and May, R. M. (1992). Evolutionary games and spatial chaos. Nature, 359(6398):826-829. 16, 17, 18, 47, 48

Nowak, M. A. and May, R. M. (1993). The spatial dilemmas of evolution. International Journal of Bifurcation and Chaos, 3(1):35-78. 48, 112

Ohtsuki, H., Hauert, C., Lieberman, E., and Nowak, M. A. (2006). A simple rule for the evolution of cooperation on graphs and social networks. Nature, 441(7092):502-505. 90, 111

Ohtsuki, H. and Nowak, M. A. (2006). The replicator equation on graphs. Journal of Theoretical Biology, 243(1):86-97. 90, 117

Ohtsuki, H., Pacheco, J. M., and Nowak, M. A. (2007). Evolutionary graph theory: breaking the symmetry between interaction and replacement. Journal of Theoretical Biology, 246(4):681-694. 17, 48, 116

Okabe, A., Boots, B., Sugihara, K., and Chiu, S. N. (2009). Spatial tessellations: concepts and applications of Voronoi diagrams, volume 501. John Wiley \& Sons. 47

Olsder, G. J. (1997). Mathematical Systems Theory. Delft University Press. 10, 11, 41

Onnela, J.-P., Arbesman, S., González, M. C., Barabási, A.-L., and Christakis, N. A. (2011). Geographic constraints on social network groups. PLoS One, 6(4). paper nr. e16939. 16

Orlando, P., Gatenby, R., and Brown, J. (2012). Cancer treatment as a game: integrating evolutionary game theory into the optimal control of chemotherapy. Physical Biology, 9(6). paper nr. 065007. 89

Osipenko, G. (2007). Dynamical Systems, Graphs, and Algorithms. Lecture Notes in Mathematics. Springer-Verlag Berlin Heidelberg. 11 
O'sullivan, D. and Torrens, P. M. (2001). Cellular models of urban systems. In Bandini, S. and Worsch, T., editors, Theory and Practical Issues on Cellular Automata, pages 108-116. Springer. 121

Parsons, S. and Wooldridge, M. (2002). Game theory and decision theory in multi-agent systems. Autonomous Agents and Multi-Agent Systems, 5(3):243-254. 10

Patel, A. P., Tirosh, I., Trombetta, J. J., Shalek, A. K., Gillespie, S. M., Wakimoto, H., Cahill, D. P., Nahed, B. V., Curry, W. T., and Martuza, R. L. (2014). Single-cell RNA-seq highlights intratumoral heterogeneity in primary glioblastoma. Science, 344(6190):13961401. 89

Perfahl, H., Byrne, H. M., Chen, T., Estrella, V., Alarcón, T., Lapin, A., Gatenby, R. A., Gillies, R. J., Lloyd, M. C., and Maini, P. K. (2011). Multiscale modelling of vascular tumour growth in 3D: the roles of domain size and boundary conditions. PloS One, 6(4). paper nr. e14790. 89

Pfeiffer, T., Schuster, S., and Bonhoeffer, S. (2001). Cooperation and competition in the evolution of ATP-producing pathways. Science, 292(5516):504-507. 9

Rand, D. G. and Nowak, M. A. (2013). Human cooperation. Trends in Cognitive Sciences, 17(8):413-425. 49

Reichenbach, T., Mobilia, M., and Frey, E. (2007). Mobility promotes and jeopardizes biodiversity in rock-paper-scissors games. Nature, 448(7157):1046-1049. 18, 47, 48, 52, $85,112,119$

Ren, D., Madsen, J. S., Sørensen, S. J., and Burmølle, M. (2015). High prevalence of biofilm synergy among bacterial soil isolates in cocultures indicates bacterial interspecific cooperation. The ISME Journal, 9(1):81-89. 84

Richards, F. (1959). A flexible growth function for empirical use. Journal of Experimental Botany, 10(2):290-301. 7

Riley, M. A. and Gordon, D. M. (1999). The ecological role of bacteriocins in bacterial competition. Trends in Microbiology, 7(3):129-133. 74

Roca, C. P., Cuesta, J. A., and Sánchez, A. (2009). Evolutionary game theory: Temporal and spatial effects beyond replicator dynamics. Physics of Life Reviews, 6(4):208-249. 15, 17,116

Ryan, C. J., Smith, M. R., De Bono, J. S., Molina, A., Logothetis, C. J., De Souza, P., Fizazi, K., Mainwaring, P., Piulats, J. M., and Ng, S. (2013). Abiraterone in metastatic prostate cancer without previous chemotherapy. New England Journal of Medicine, 368(2):138-148. 91

Sabelis, M. (1991). Life-history evolution of spider mites. In Schuster, R. and Murphy, P. W., editors, The Acari, chapter 2, pages 23-49. Springer, Dordrecht. 36 
Sabelis, M. W. and Janssen, A. (1994). Evolution of life-history patterns in the phytoseiidae. In Houck, M. A., editor, Mites, chapter 4, pages 70-98. Springer. 36

Sabelis, M. W. and Overmeer, W. (1987). The laboratory observations on the predatory mites and fruit-tree red spider mites. Unpublished data. 39

Santos, F. C. and Pacheco, J. M. (2005). Scale-free networks provide a unifying framework for the emergence of cooperation. Physical Review Letters, 95(9):098104. 15

Santos, M. D., Pinheiro, F. L., Santos, F. C., and Pacheco, J. M. (2012). Dynamics of nperson snowdrift games in structured populations. Journal of Theoretical Biology, 315:8186. 47

Schweitzer, F., Behera, L., and Mühlenbein, H. (2002). Evolution of cooperation in a spatial prisoner's dilemma. Advances in Complex Systems, 5(02n03):269-299. 17

Simpson, M. J., Merrifield, A., Landman, K. A., and Hughes, B. D. (2007). Simulating invasion with cellular automata: connecting cell-scale and population-scale properties. Physical Review E, 76(2). paper nr. 021918. 18, 112

Singh, J. and Marx, M. (2013). Geographic constraints on knowledge spillovers: Political borders vs. spatial proximity. Management Science, 59(9):2056-2078. 16

Sonnenberg, G. F., Monticelli, L. A., Alenghat, T., Fung, T. C., Hutnick, N. A., Kunisawa, J., Shibata, N., Grunberg, S., Sinha, R., and Zahm, A. M. (2012). Innate lymphoid cells promote anatomical containment of lymphoid-resident commensal bacteria. Science, 336(6086):1321-1325. 73

Sottoriva, A., Spiteri, I., Piccirillo, S. G., Touloumis, A., Collins, V. P., Marioni, J. C., Curtis, C., Watts, C., and Tavaré, S. (2013). Intratumor heterogeneity in human glioblastoma reflects cancer evolutionary dynamics. Proceedings of the National Academy of Sciences, 110(10):4009-4014. 89

Staňková, K., Abate, A., and Sabelis, M. W. (2013a). Intra-seasonal strategies based on energy budgets in a dynamic predator-prey game. In Křivan, V. and Zaccour, G., editors, Advances in Dynamic Games, pages 205-222. Springer. 19, 39, 41, 42, 116, 123

Staňková, K., Abate, A., and Sabelis, M. W. (2013b). Irreversible prey diapause as an optimal strategy of a physiologically extended Lotka-Volterra model. Journal of Mathematical Biology, 66(4):767-794. 41, 42

Swanton, C. (2012). Intratumor heterogeneity: evolution through space and time. Cancer Research, 72(19):4875-4882. 89

Sysi-Aho, M., Saramäki, J., Kertész, J., and Kaski, K. (2005). Spatial snowdrift game with myopic agents. The European Physical Journal B-Condensed Matter and Complex Systems, 44(1):129-135. 17, 47 
Szabó, G. and Tőke, C. (1998). Evolutionary prisoner's dilemma game on a square lattice. Physical Review E, 58(1). paper nr. 69. 17, 47, 48

Számadó, S., Szalai, F., and Scheuring, I. (2008). The effect of dispersal and neighbourhood in games of cooperation. Journal of Theoretical Biology, 253(2):221-227. 90

Tajfel, H. (2010). Social Identity and Intergroup Relations. Cambridge University Press. 16

Tauber, M. J., Tauber, C. A., and Masaki, S. (1986). Seasonal Adaptations of Insects. Oxford University Press. 25, 26

Taylor, P. D. and Jonker, L. B. (1978). Evolutionary stable strategies and game dynamics. Mathematical Biosciences, 40(1-2):145-156. 12

Thalhauser, C. J., Lowengrub, J. S., Stupack, D., and Komarova, N. L. (2010). Selection in spatial stochastic models of cancer: migration as a key modulator of fitness. Biology Direct, 5(1):11-21. 89

Thoemmes, M. S., Fergus, D. J., Urban, J., Trautwein, M., and Dunn, R. R. (2014). Ubiquity and diversity of human-associated demodex mites. PloS One, 9(8). paper nr. e106265. 73

Tilman, D. and Kareiva, P. M. (1997). Spatial Ecology: the Role of Space in Population Dynamics and Interspecific Interactions. Princeton University Press. 102

Tomasetti, C., Vogelstein, B., and Parmigiani, G. (2013). Half or more of the somatic mutations in cancers of self-renewing tissues originate prior to tumor initiation. Proceedings of the National Academy of Sciences, 110(6):1999-2004. 89

Tomlinson, I. (1997). Game-theory models of interactions between tumour cells. European Journal of Cancer, 33(9):1495-1500. 90

Traulsen, A., Claussen, J. C., and Hauert, C. (2005). Coevolutionary dynamics: from finite to infinite populations. Physical Review Letters, 95(23). paper nr. 238701. 15

Tsai, H., Penson, D. F., Makambi, K. H., Lynch, J. H., Van Den Eeden, S. K., and Potosky, A. L. (2013). Efficacy of intermittent androgen deprivation therapy vs conventional continuous androgen deprivation therapy for advanced prostate cancer: a meta-analysis. Urology, 82(2):327-334. 91

Uboh, F., Asuquo, E., Eteng, M., and Akpanyung, E. (2011). Endosulfan-induces renal toxicity independent of the route of exposure in rats. American Journal of Biochemistry and Molecular Biology, 1(4):359-367. 125

Upton, G. and Cook, I. (2014). A Dictionary of Statistics. Oxford University Press. 102

Uyttendaele, P. and Thuijsman, F. (2015). Evolutionary games and local dynamics. International Game Theory Review, 17(02). paper nr. 1540016. 47, 48, 69, 90 
Uyttendaele, P., Thuijsman, F., Collins, P., Peeters, R., Schoenmakers, G., and Westra, R. (2012). Evolutionary games and periodic fitness. Dynamic Games and Applications, $2(3): 335-345.90$

Veerman, A. (1985). Diapause. In Helle, W. and Sabelis, M. W., editors, Spider Mites: Their Biology, Natural Enemies and Control, volume 1A of World Crop Pests, pages 279-316. Elsevier Science Publishers, Amsterdam, The Netherlands. 25, 26

Veerman, A. (1992). Diapause in phytoseiid mites: A review. Experimental and Applied Acarology, 14(1):1-60. 25, 26

Veerman, A. (1994). Photoperiodic and thermoperiodic control of diapause in plantinhabiting mites: A Review. Netherlands Journal of Zoology, 44(1):139-155. 25, 26

Verhoef, H. A. and Morin, P. J. (2010). Community Ecology: Processes, Models, and Applications. Oxford University Press. 73

Vetsigian, K., Jajoo, R., and Kishony, R. (2011). Structure and evolution of streptomyces interaction networks in soil and in silico. PLoS Biology, 9(10). paper nr. e1001184. 74

Volterra, V. (1927). Variazioni e fluttuazioni del numero d'individui in specie animali conviventi. Accademia dei Lincei. 14, 25

Von Neumann, J. and Morgenstern, O. (1944). Theory of Games and Economic Behavior. Princeton University Press. 1

Waclaw, B., Bozic, I., Pittman, M. E., Hruban, R. H., Vogelstein, B., and Nowak, M. A. (2015). A spatial model predicts that dispersal and cell turnover limit intratumour heterogeneity. Nature, 525(7568):261-264. 89

Wakano, J. Y. and Hauert, C. (2011). Pattern formation and chaos in spatial ecological public goodsgames. Journal of Theoretical Biology, 268(1):30-38. 16

Wakano, J. Y., Nowak, M. A., and Hauert, C. (2009). Spatial dynamics of ecological public goods. Proceedings of the National Academy of Sciences, 106(19):7910-7914. 15

Wang, M., Huang, Y., and Wu, Z. (2016). Simulation of yeast cooperation in 2D. Bulletin of Mathematical Biology, 78(3):531-555. 49

Wang, W.-X., Lai, Y.-C., and Grebogi, C. (2010). Effect of epidemic spreading on species coexistence in spatial rock-paper-scissors games. Physical Review E, 81(4). paper nr. 046113. 16

Waters, C. M. and Bassler, B. L. (2005). Quorum sensing: cell-to-cell communication in bacteria. Annual Review of Cell and Developmental Biology, 21:319-346. 75

Weissing, F. J. (1991). Evolutionary stability and dynamic stability in a class of evolutionary normal form games. In Selten, R., editor, Game Equilibrium Models I, pages 29-97. Springer. 52, 53 
Werner, B., Lutz, D., Brümmendorf, T. H., Traulsen, A., and Balabanov, S. (2011). Dynamics of resistance development to Imatinib under increasing selection pressure: a combination of mathematical models and in vitro data. PloS One, 6(12). paper nr. e28955. 89

Williams, P., Winzer, K., Chan, W. C., and Camara, M. (2007). Look who's talking: communication and quorum sensing in the bacterial world. Philosophical Transactions of the Royal Society B: Biological Sciences, 362(1483):1119-1134. 75

Williamson, J. G. (1988). Migration and urbanization. Handbook of Development Economics, 1:425-465. 120

World Health Organization (2016). Global Report on Urban Health: Equitable Healthier Cities for Sustainable Development. World Health Organization. 120

World Health Organization (2017). Antibacterial Agents in Clinical Development: Analysis of the Antibacterial Clinical Development Pipeline including Tuberculosis. World Health Organization. 126

Yao, G. L., Albertsen, P. C., Moore, D. F., Shih, W., Lin, Y., DiPaola, R. S., and Yao, S. L. (2008). Survival following primary androgen deprivation therapy among men with localized prostate cancer. JAMA, 300(2):173-181. 91

Yearsley, J. M. (2004). Transient population dynamics and short-term sensitivity analysis of matrix population models. Ecological Modeling, 177(3):245-258. 49

You, L., Brown, J. S., Thuijsman, F., Cunningham, J. J., Gatenby, R. A., Zhang, J., and Staňková, K. (2017). Spatial vs. non-spatial eco-evolutionary dynamics in a tumor growth model. Journal of Theoretical Biology, 435:78-97. 15, 49

Zeeman, E. C. (1980). Population dynamics from game theory. In Nitecki, Z. and Robinson, C., editors, Global Theory of Dynamical Systems, pages 471-497. Springer. 13, 50, 52

Zhang, J., Cunningham, J. J., Brown, J. S., and Gatenby, R. A. (2017). Integrating evolutionary dynamics into treatment of metastatic castrate-resistant prostate cancer. Nature Communications, 8(1). paper nr. 1816. 109, 153, 154, 155

Zhang, J., Fujimoto, J., Zhang, J., Wedge, D. C., Song, X., Zhang, J., Seth, S., Chow, C. W., Cao, Y., and Gumbs, C. (2014). Intratumor heterogeneity in localized lung adenocarcinomas delineated by multiregion sequencing. Science, 346(6206):256-259. 89

Zhang, K. H. and Shunfeng, S. (2003). Rural-urban migration and urbanization in china: Evidence from time-series and cross-section analyses. China Economic Review, 14(4):386400. 120

Zietsch, B. P., Kuja-Halkola, R., Walum, H., and Verweij, K. J. (2014). Perfect genetic correlation between number of offspring and grandoffspring in an industrialized human population. Proceedings of the National Academy of Sciences, 111(3):1032-1036. 7, 48 


\section{Supplement of Chapter 1}

This appendix shows the mathematical equivalence between replicator dynamics over $n$ types and Lotka-Volterra dynamics over $n-1$ types. Here our calculations are based on Hofbauer (1981).

Lotka-Volterra dynamics $\Rightarrow$ replicator dynamics The general form of the LotkaVolterra dynamics over $n-1$ types is given as

$$
\dot{y}_{i}=y_{i}\left(r_{i}+\sum_{j=1}^{n-1} a_{i j} y_{j}\right), \quad i=1, \ldots, n-1,
$$

where $r_{i}$ denotes the intrinsic growth rate (a constant) of type $i, y(t)=\left(y_{1}(t), \ldots, y_{n-1}(t)\right) \in$ $\mathbb{R}^{n-1}$ denotes the vector of population sizes of types at a given time $t \in[0,+\infty)$. We write $y=\left(y_{1}, \ldots, y_{n-1}\right)$ instead by omitting writing time variable $t$ in the equations to follow. Every entry of $A=\left(a_{i j}\right)$ represents the effect of type $j \in\{1,2, \ldots, n-1\}$ on type $i$ (i.e., type $j$ is enhancing, neutral to, or inhibiting the density growth of type $i$ if $a_{i j}$ is positive, zero, or negative, respectively). Here every $a_{i j}$ is a constant.

If we agree to increase the dimension of matrix $A$ to $n \times n$ by including the intrinsic growth rate $r_{1}, \ldots, r_{n-1}$, we obtain the matrix

$$
A^{\prime}=\left(\begin{array}{cccc}
a_{11} & \cdots & a_{1(n-1)} & r_{1} \\
\vdots & \ddots & \vdots & \vdots \\
a_{(n-1) 1} & \cdots & a_{(n-1)(n-1)} & r_{n-1} \\
0 & \cdots & 0 & 0
\end{array}\right) .
$$


Notice that matrix $A$ is part of $A^{\prime}$, i.e., $a_{i j}=a_{i j}^{\prime}$ for every $i, j \in\{1, \ldots, n-1\}$, and $a_{n i}=0$ for $i \in\{1, \ldots, n\}$.

Let us compactify the space of (A.1) - We introduce homogeneous coordinates by setting $y_{n}=1$ (the same approach as Hofbauer (1981)). Now we can write equation (A.1) as

$$
\begin{aligned}
\dot{y}_{i} & =y_{i}\left(a_{i n}^{\prime}+\sum_{j=1}^{n-1} a_{i j} y_{j}\right), \\
& =y_{i} \sum_{j=1}^{n} a_{i j}^{\prime} y_{j}, \quad i=1, \ldots, n .
\end{aligned}
$$

To obtain replicator dynamics we need to convert population sizes of types into frequencies of types. Let us define the frequency distribution of types as $x(t)=\left(x_{1}(t), \ldots, x_{n}(t)\right) \in \mathbb{R}^{n}$ where $x_{i}(t)$ denotes the frequency of type $i$ within the total population (of all types) at time $t$ and where $\sum_{i=1}^{n} x_{i}(t)=1$. We will write $x=\left(x_{1}, \ldots, x_{n}\right) \in \mathbb{R}^{n}$ instead. We move to

$$
x_{i}=\frac{y_{i}}{\sum_{j=1}^{n} y_{j}}, \quad i=1, \ldots, n,
$$

and here we assume the total population of all types is non-zero at any given moment, i.e., $\sum_{j=1}^{n} y_{j}>0$ for $t \in[0,+\infty)$.

Further, equation (A.3) indicates

$$
x_{n}=\frac{1}{\sum_{j=1}^{n} y_{j}},
$$

and

$$
y_{i}=\frac{x_{i}}{x_{n}}, \quad i=1, \ldots, n .
$$

We write the derivative of $x_{i}$ from equation (A.3) as follows:

$$
\begin{array}{r}
\dot{x}_{i}=\frac{\dot{y}_{i} \sum_{j=1}^{n} y_{j}-y_{i} \sum_{j=1}^{n} \dot{y}_{j}}{\left(\sum_{j=1}^{n} y_{j}\right)^{2}}, \\
=\frac{\dot{y}_{i}}{\sum_{j=1}^{n} y_{j}}-\frac{y_{i} \sum_{j=1}^{n} \dot{y}_{j}}{\left(\sum_{j=1}^{n} y_{j}\right)^{2}} .
\end{array}
$$


Using equations (A.4) and (A.2), we continue calculating the derivative of $x_{i}$ :

$$
\begin{aligned}
\dot{x}_{i} & =x_{n} \dot{y}_{i}-x_{n}^{2} y_{i} \sum_{j=1}^{n} \dot{y}_{j}, \\
& =x_{n} y_{i} \sum_{j=1}^{n} a_{i j}^{\prime} y_{j}-x_{n}^{2} y_{i} \sum_{j=1}^{n} \sum_{k=1}^{n} y_{j} a_{j k}^{\prime} y_{k}, \\
& =x_{n} y_{i} \sum_{j=1}^{n} a_{i j}^{\prime} \frac{x_{j}}{x_{n}}-x_{n}^{2} y_{i} \sum_{j=1}^{n} \sum_{k=1}^{n} \frac{x_{j}}{x_{n}} a_{j k}^{\prime} \frac{x_{k}}{x_{n}}, \\
& =\frac{x_{i}}{x_{n}}\left(\sum_{j=1}^{n} a_{i j}^{\prime} x_{j}-\sum_{j=1}^{n} \sum_{k=1}^{n} x_{j} a_{j k}^{\prime} x_{k}\right), \\
& =\frac{x_{i}}{x_{n}}\left(e_{i} A^{\prime} x^{\top}-x A^{\prime} x^{\top}\right), \quad \text { for } i=1, \ldots, n,
\end{aligned}
$$

where $e_{i}$ is the $i$-th row of an $n \times n$ identity matrix. Notice that in (A.7) the term $\frac{1}{x_{n}}$ is positive (i.e., $\frac{1}{x_{n}}=\sum_{j=1}^{n} y_{j}$ while we assume total population of all types is always positive). If we assume the positive factor $\frac{1}{x_{n}}$ means a change in velocity with which the solution of the system (A.7) moves, we can remove the term $\frac{1}{x_{n}}$. In other words the trajectory in $x$ is given by

$$
\dot{x}_{i}=x_{i}\left(e_{i} A^{\prime} x^{\top}-x A^{\prime} x^{\top}\right), \quad \text { for } i=1, \ldots, n
$$

Hence, we have converted the Lotka-Volterra model (1.7) over $n-1$ types into the replicator dynamics (1.4) over $n$ types.

Replicator dynamics $\Rightarrow$ Lotka-Volterra dynamics Suppose a population is composed of $n$ types. The general form of replicator dynamics is given by

$$
\dot{x}_{i}=x_{i}\left(f_{i}(x)-\bar{f}(x)\right) \quad i=1, \ldots, n,
$$

where $x=\left(x_{1}, \ldots, x_{n}\right) \in \mathbb{R}^{n}$ with $x_{i} \geq 0$ is the frequency of type $i$ and $\sum_{i=1}^{n} x_{i}=1$, the fitness of type $i$ is $f_{i}(x)$ and the average fitness per type is $\bar{f}(x)=\sum_{i=1}^{n} x_{i} f_{i}(x)$.

Next we introduce two preliminaries that are useful for our converting calculations:

- If we add a function $h=h\left(x_{i}\right)$ to all the $f_{i}(x)$ 's, this has the effect of replacing $\bar{f}(x)$ by $\bar{f}(x)+h$ since $h=\sum_{i=1}^{n} x_{i} h$. Thus the right-hand side of equation (A.8) does not change, i.e., $\dot{x}_{i}=x_{i}\left(f_{i}(x)+h-\bar{f}(x)-h\right)=x_{i}\left(f_{i}(x)-\bar{f}(x)\right)$ for $i=1, \ldots, n$. Now assume the game is described by an $n \times n$ fitness matrix $A$, then

$$
f_{i}=e_{i} A x^{\top}, \quad \text { for } i=1, \ldots, n .
$$

If we add a constant $c$ to all the entries in the $j$-th column of $A$ (where $j=1, \ldots, n$ ), this has the effect of adding the function $c x_{j}$ to all the $f_{i}$ 's, so replicator dynamics (A.8) do not change in this way. In particular, this means that, by subtracting the entry in the last row ( $n$-th row) from each column, we can assume that the payoff matrix $A$ has all entries in the bottom row as 0's, without changing the replicator dynamics (A.8). 
- Equations (A.8) can be used for calculating $\frac{d}{d t}\left(\frac{x_{i}}{x_{j}}\right)$ :

$$
\begin{aligned}
\frac{d}{d t}\left(\frac{x_{i}}{x_{j}}\right) & =\frac{\dot{x}_{i} x_{j}-x_{i} \dot{x}_{j}}{x_{j}^{2}}, \\
& =\frac{x_{i}\left(f_{i}(x)-\bar{f}(x)\right) x_{j}-x_{i} x_{j}\left(f_{j}(x)-\bar{f}(x)\right)}{x_{j}^{2}}, \\
& =\frac{x_{i}}{x_{j}}\left(f_{i}(x)-f_{j}(x)\right), \quad \text { for } i, j=1, \ldots, n .
\end{aligned}
$$

To obtain Lotka-Volterra dynamics we need population sizes of types: Let $y=\left(y_{1}, \ldots, y_{n}\right) \in \mathbb{R}^{n}$ denote the vector of population sizes of types and $y_{i}$ the population size of type $i$ (and it can either be a non-negative ratio or number), then

$$
x_{i}=\frac{y_{i}}{\sum_{j=1}^{n} y_{j}} .
$$

where we assume the total population size including all types is always positive.

Let $y_{n}=1$ then

$$
x_{n}=\frac{1}{\sum_{j=1}^{n} y_{j}}
$$

and

$$
y_{i}=x_{i} \sum_{j=1}^{n} y_{j}=\frac{x_{i}}{x_{n}} .
$$

Lending this equality (A.10) we can write equation (A.12) as

$$
\dot{y}_{i}=y_{i}\left(f_{i}(x)-f_{n}(x)\right) .
$$

Suppose further we have chosen $A$ to have its bottom row all 0's (i.e., which we can do without changing the equations). Then $f_{n} \equiv 0$. Equation (A.13) becomes

$$
\begin{aligned}
\dot{y}_{i} & =y_{i} f_{i}(x), \\
& =y_{i} e_{i} A x^{\top}, \\
& =y_{i} \sum_{j=1}^{n} a_{i j} x_{j}, \\
& =y_{i}\left(\sum_{j=1}^{n-1} a_{i j} x_{j}+a_{i n} x_{n}\right), \\
& =y_{i}\left(\sum_{j=1}^{n-1} a_{i j} y_{j} x_{n}+a_{i n} x_{n}\right), \\
& =x_{n} y_{i}\left(a_{i n}+\sum_{j=1}^{n-1} a_{i j} y_{j}\right) .
\end{aligned}
$$

If we assume the positive factor $x_{n}$ means a change in velocity with which the solution of the system (A.14) moves, we can remove the term $x_{n}$. In other words, the trajectory in $y$ is given by

$$
\dot{y}_{i}=y_{i}\left(r_{i}+\sum_{j=1}^{n-1} a_{i j} y_{j}\right), \quad \text { for } i=1, \ldots, n-1,
$$


where $r_{i}=a_{i n}$. Hence, we have converted the replicator dynamics (1.4) over $n$ types into the Lotka-Volterra dynamics (1.7) over $n-1$ types. 


\section{APPENDIX}

\section{Supplement of Chapter 3}

In this appendix we show plots of frequency dynamics, produced by continuous-space and discrete-space models in two different case studies.

\section{B.1 Results with respect to varying radii}

This section relates to Chapter 3 and Case study 2, where we examined the impact of varying interaction radius on frequency dynamics, which are achieved in the following spatial models: the continuous-space model and the discrete-space models (with the "no placement", "stochastic placement" and "deterministic placement" scenarios, respectively).

Figures B.1, B.2 and B.3 show the frequency dynamics of matrices $A_{1}^{+}, A_{0}^{+}$and $A_{-1}^{+}$, respectively. Each subfigure shows the frequency dynamics for a different spatial game and a different interaction radius $(1,2,10$ and 100). For this case study, individuals have a lifespan of one generation.

\section{B.2 Results with respect to increased lifespan}

This section relates to Chapter 3 and Case study 3, where we examined the impact of increased lifespan on frequency dynamics, which are achieved in the following spatial models: the continuous-space model and the discrete-space models (with the "no placement", "stochastic placement" and "deterministic placement" scenarios, respectively).

Figures B.4, B.5 and B.6 show the frequency dynamics of matrices $A_{1}^{+}, A_{0}^{+}$and $A_{-1}^{+}$, 


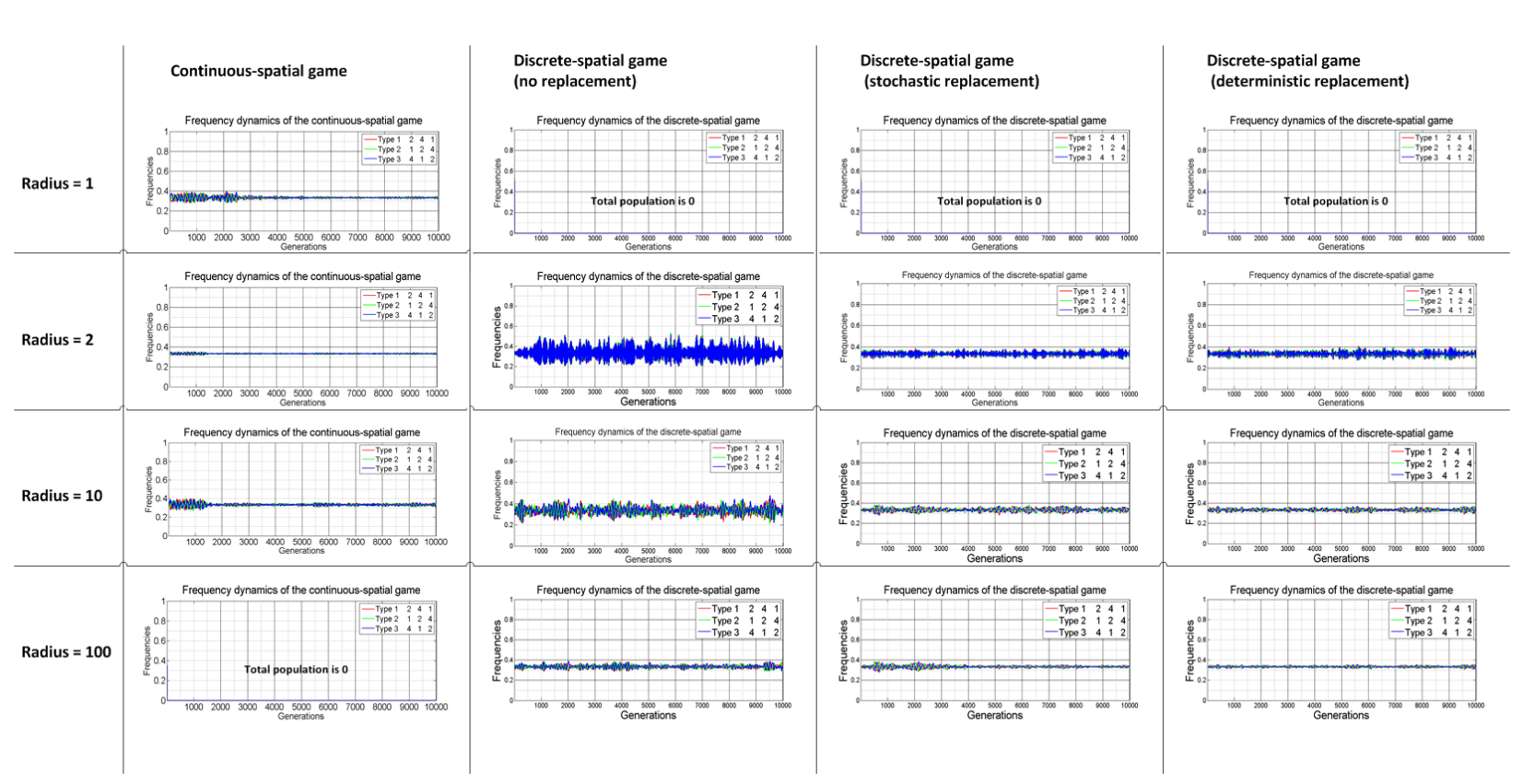

Figure B.1: The frequency dynamics for the game with matrix $A_{1}^{+}$for different interaction radii (rows) and different spatial models (columns). Each subfigure shows the frequency dynamics averaged over 15 runs while the frequency dynamics were achieved after 10000 generations per run. Here lifespan is 1 generation.

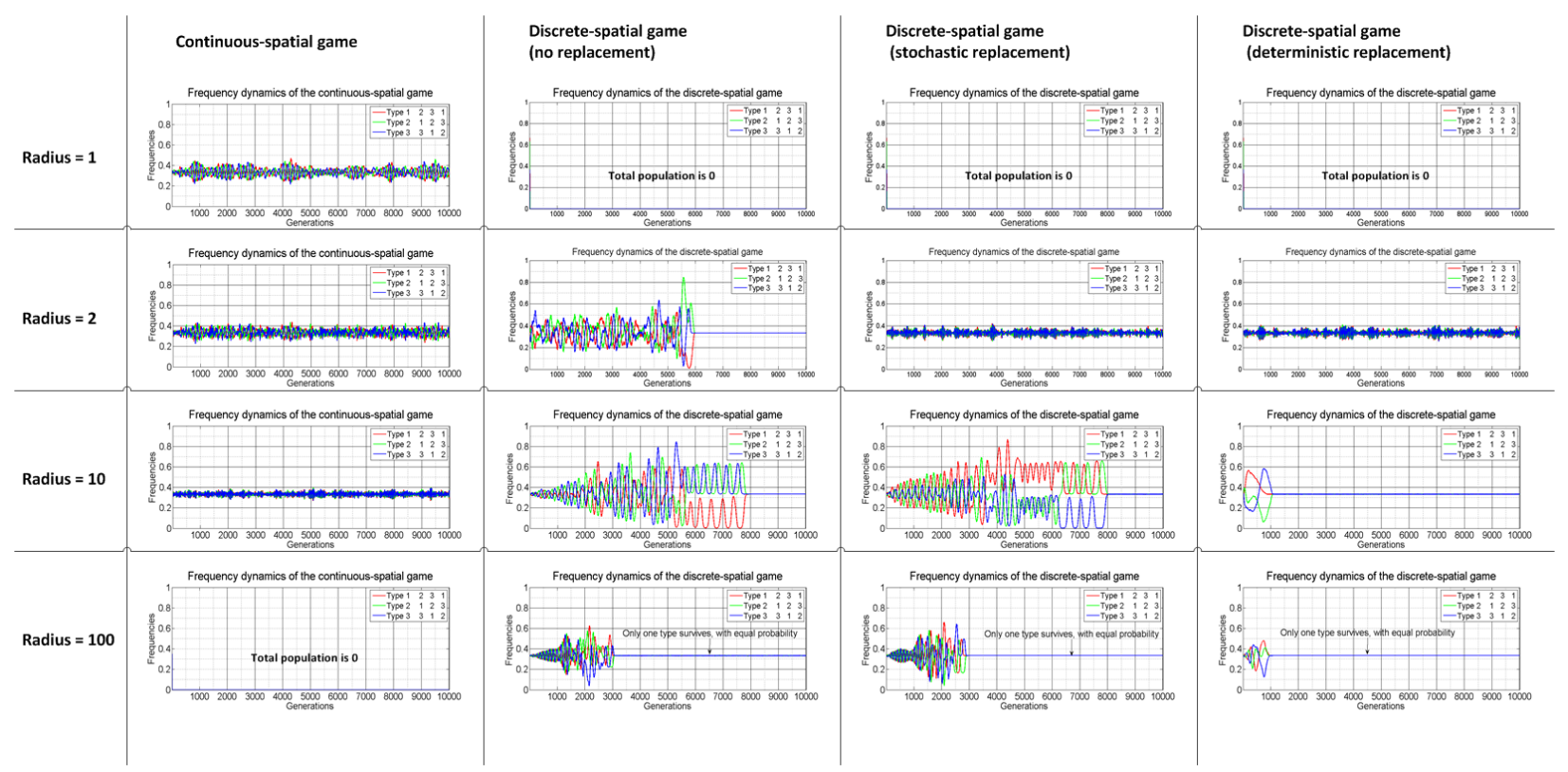

Figure B.2: The frequency dynamics for the game with matrix $A_{0}^{+}$for different interaction radii (rows) and different spatial models (columns). Each subfigure shows the frequency dynamics averaged over 15 runs while the frequency dynamics were achieved after 10000 generations per run. Here lifespan is 1 generation.

respectively. Each subfigure shows the frequency dynamics for a different spatial game and a different interaction radius $(1,2,10$ and 100). For this case study, individuals have a lifespan of five generations. 


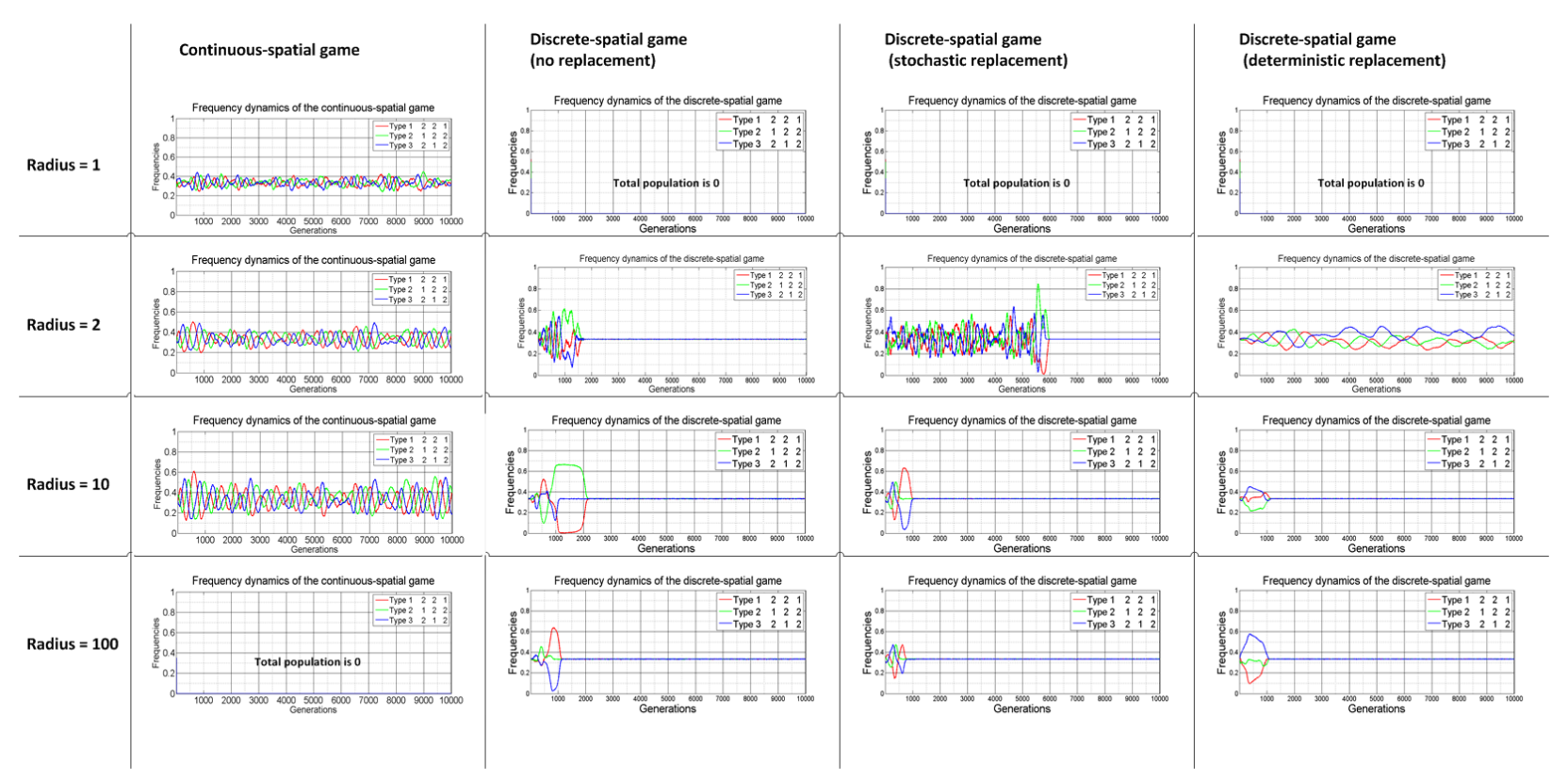

Figure B.3: The frequency dynamics for the game with matrix $A_{-1}^{+}$for different interaction radii (rows) and different spatial models (columns). Each subfigure shows the frequency dynamics averaged over 15 runs while the frequency dynamics were achieved after 10000 generations per run. Here lifespan is 1 generation.

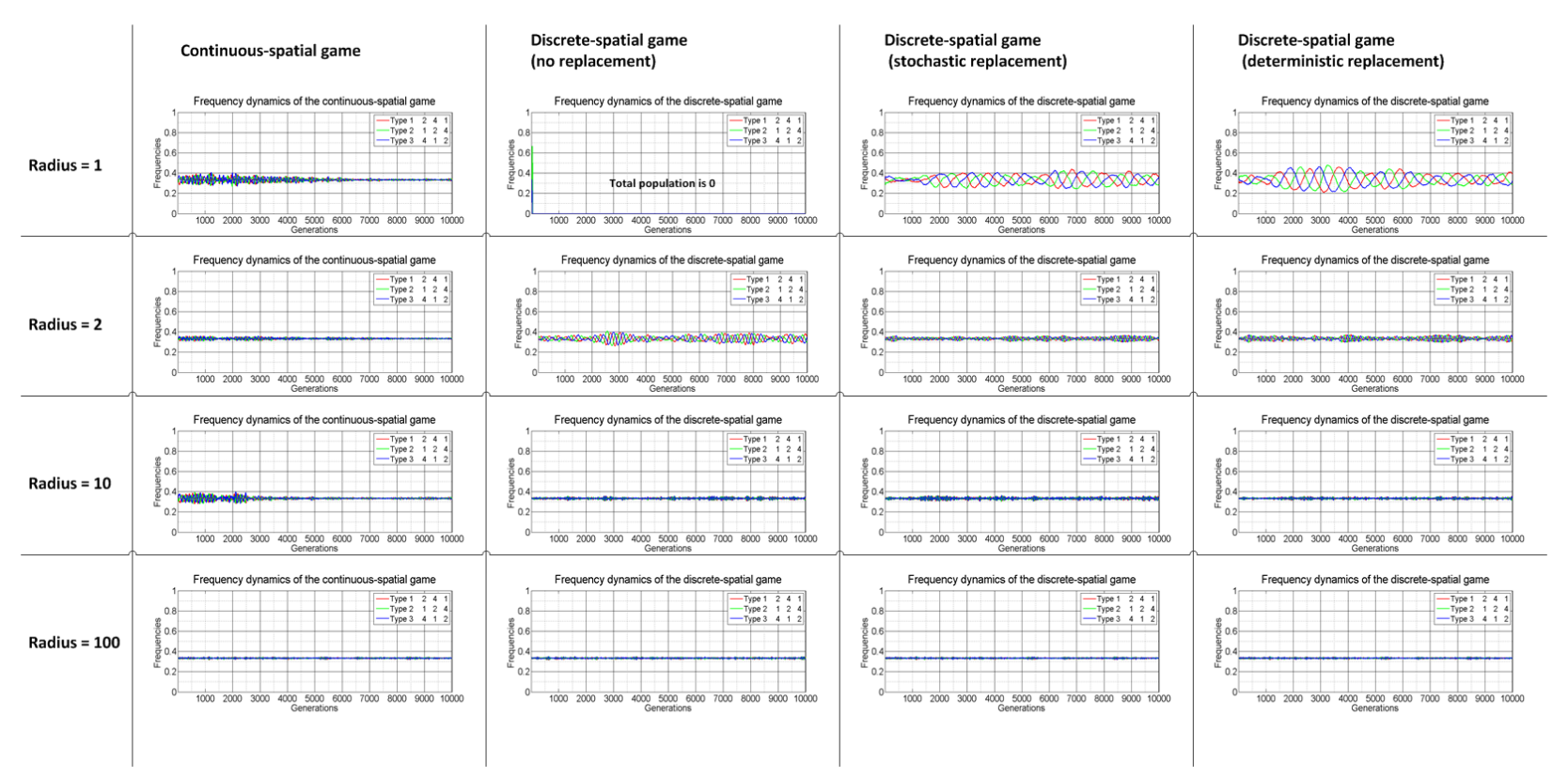

Figure B.4: The frequency dynamics for the game with matrix $A_{1}^{+}$for different interaction radii (rows) and different spatial models (columns). Each subfigure shows the frequency dynamics averaged over 15 runs while the frequency dynamics were achieved after 10000 generations per run. Here lifespan is 5 generations. 


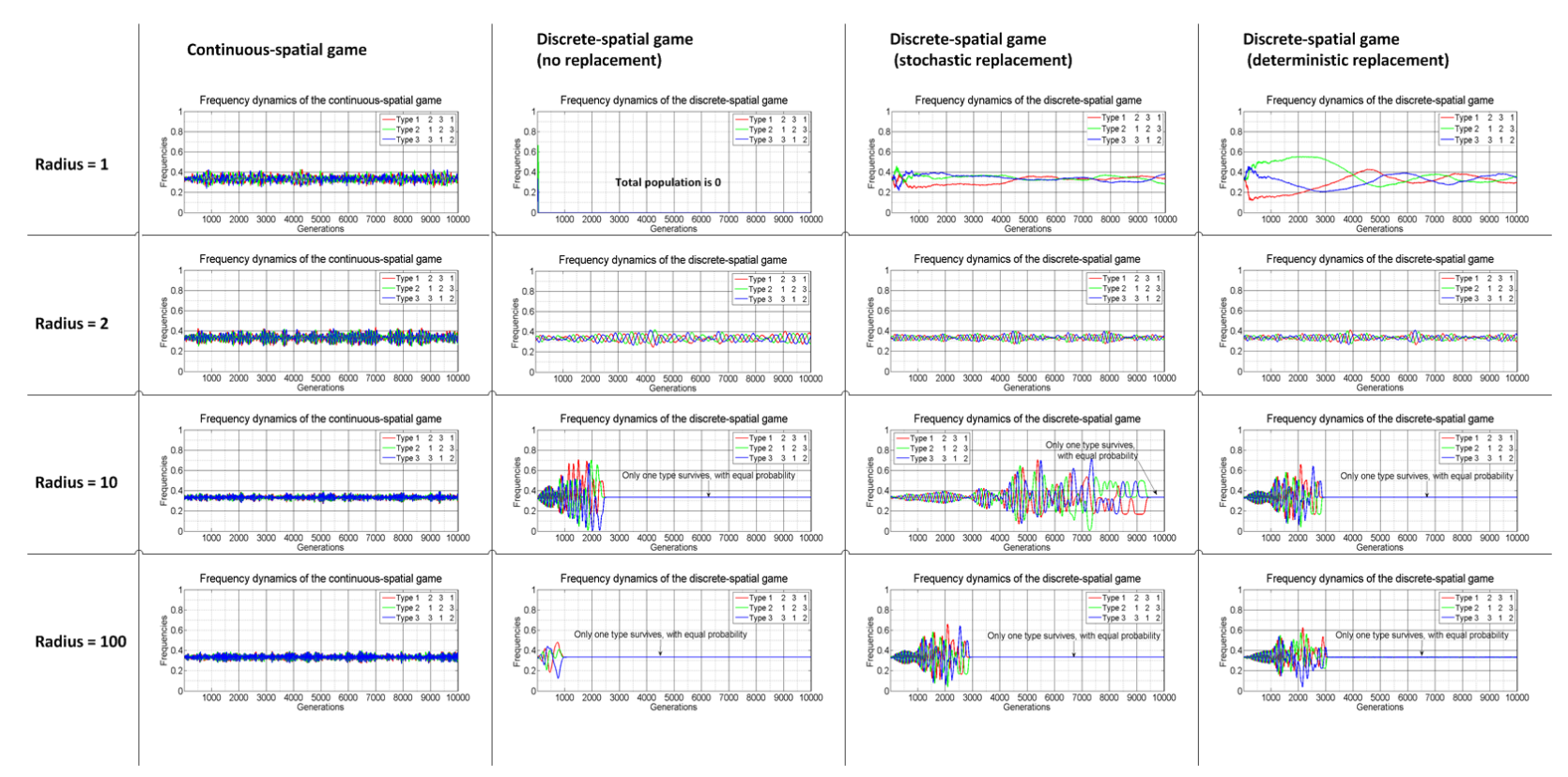

Figure B.5: The frequency dynamics for the game with matrix $A_{0}^{+}$for different interaction radii (rows) and different spatial models (columns). Each subfigure shows the frequency dynamics averaged over 15 runs while the frequency dynamics were achieved after 10000 generations per run. Here lifespan is 5 generations.

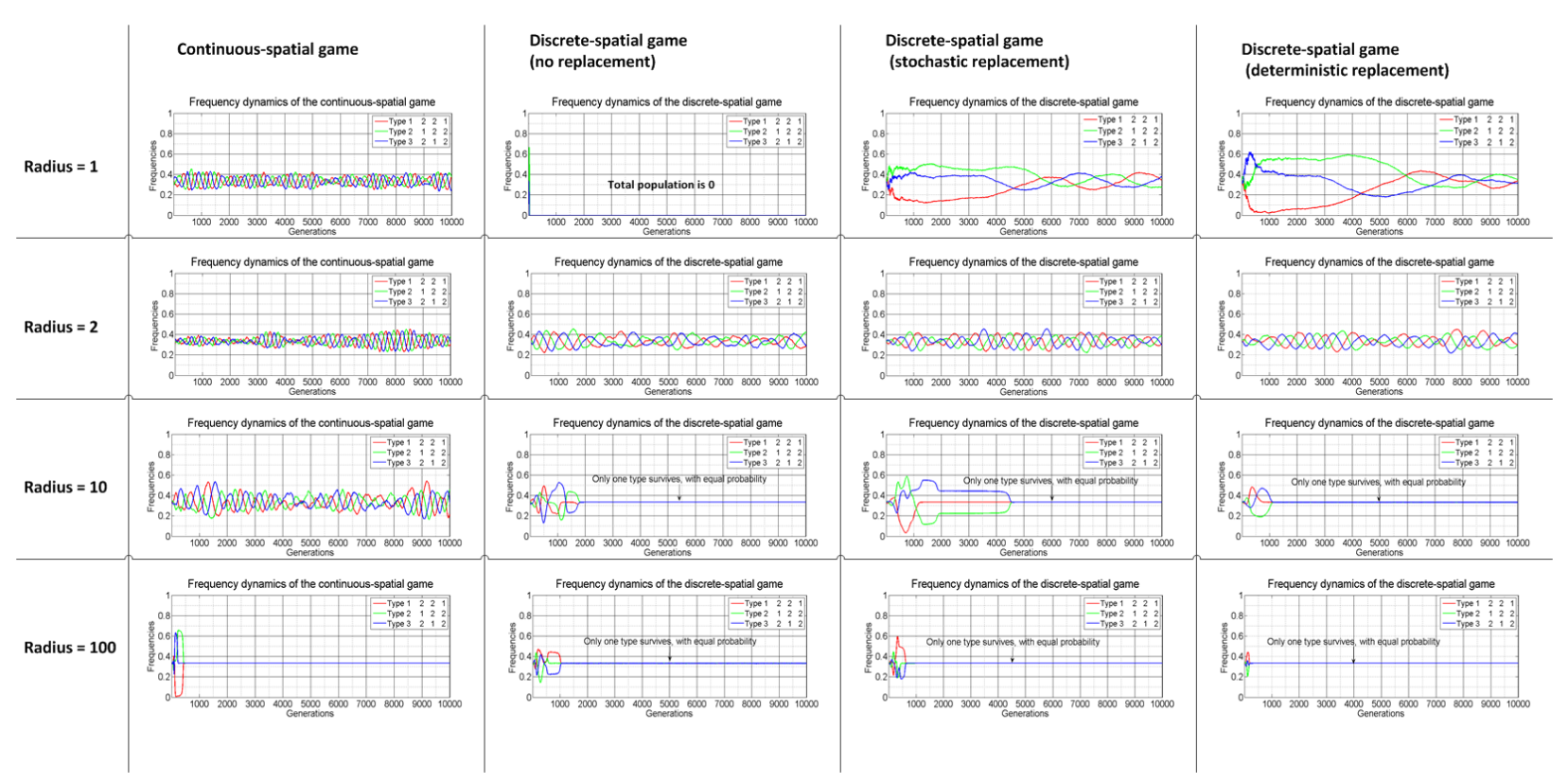

Figure B.6: The frequency dynamics for the game with matrix $A_{-1}^{+}$for different interaction radii (rows) and different spatial models (columns). Each subfigure shows the frequency dynamics averaged over 15 runs while the frequency dynamics were achieved after 10000 generations per run. Here lifespan is 5 generations. 


\section{Supplement of Chapter 5}

This appendix provides supplementary materials for Chapter 5 listed as follows.

\section{C.1 The nonspatial model}

In this section we introduce a nonspatial model of metastatic castrate-resistant prostate cancer (mCRPC) from Zhang et al. (2017) that we compare our spatial model to. When individuals interact randomly with others over the entire field, the saturated equilibrium strategy frequencies of our spatial game match those of the nonspatial ESS. In general, the replicator dynamics (Hofbauer and Sigmund, 1998) represent one of the dynamics which, under certain conditions, converge to the ESS.

For each cell type $i \in T$, the replicator dynamics define the time change $\dot{x}_{i}$ of cell frequency $x_{i}$ :

$$
\dot{x}_{i}=x_{i}\left(e_{i} A x^{\top}-x A x^{\top}\right), \quad i \in T
$$

with $x(0)=(1 / 3,1 / 3,1 / 3)$. Here matrix $A$ is the fitness (or payoff) matrix, $x=\left(x_{T^{+}}, x_{T^{P}}, x_{T^{-}}\right)$ is a vector of cell type frequencies, and $e_{i}$ is the $i$-th row of a $3 \times 3$ identity matrix. Each element $a_{i j}$ of the fitness matrix $A=\left(a_{i j}\right)$ defines a probability that a cell of type $i$ will produce a daughter cell of the same type when interacting with a cell of type $j \in T$. The nonspatial model (C.1) assumes that the population of cells is well mixed and, therefore, a probability that a cell of type $i$ meets a cell of type $j$ at time $t$ is given by the frequency of the $j$ cells in the entire population at time $t$. 
We assume that each cell type competes most with its own type. Intra-type interactions do not increase proliferation rates. For this reason, we have set the diagonal elements of $A$ equal to 0 to reflect this lack of effect. The off-diagonal elements are positive (but less than 1) to reflect the lower competition between cell types and the gains that can accrue to a cell type from interactions with an alternative cell type. Standardizing the elements so that the off-diagonal elements are 0 may introduce a possible artifact. In the spatial model these elements are the probability of proliferating when two cells interact, and hence no proliferation can occur when two cells of the same type interact. Thus, at least two cell types must be present for the tumor to grow. Frequency-dependent processes favor the coexistence of the diverse cell types even as density-dependence limits the overall population size of cancer cells within the space. In Section C.4 we show that having 0 elements on the diagonal of the matrix does not influence any of the conclusions qualitatively.

Therefore, $A$ has the form

\begin{tabular}{l|lll} 
& $T^{+}$ & $T^{P}$ & $T^{-}$ \\
\hline$T^{+}$ & 0 & $a$ & $b$ \\
$T^{P}$ & $c$ & 0 & $d$ \\
$T^{-}$ & $e$ & $f$ & 0
\end{tabular}.

The rest of the elements of matrix $A$ are assumed to be distinct from each other and from the interval $(0,1)$.

From pairwise experiments in vitro and from the properties of the individual types of cancer cells we derive the following inequalities regarding the coefficients $a-f$ (Zhang et al., 2017): $a>f, c>e, b<d, a>b, c>d$, and $e>f$.

The first three inequalities are based on observations regarding which cell type receives the greater benefit when interacting with a particular cell type:

- $a>f: \mathrm{A} T^{+}$cell profits more than a $T^{-}$cell from interacting with a $T^{P}$ cell, because a $T^{P}$ cell produces a systematic testosterone that a $T^{+}$cell needs for proliferation. When testosterone is available a $T^{+}$cell is expected to have a higher proliferation rate than a $T^{-}$cell.

- $c>e: \mathrm{A} T^{+}$cell creates a cellular infrastructure that a $T^{P}$ cell benefits from. Therefore, we expect that a $T^{P}$ cell is more fit than a $T^{-}$cell when interacting with a $T^{+}$cell.

- $b<d$ : When interacting with a $T^{-}$cell, a $T^{+}$cell receives no testosterone to proliferate, while a $T^{P}$ cell may profit from less competition for resources.

The rest of the inequalities are based on observations regarding which cancer cell type provides more benefit to a particular cell type:

- $a>b$ : A $T^{+}$cell has a higher chance to proliferate when interacting with a $T^{P}$ cell than when interacting with a $T^{-}$cell, because a $T^{P}$ cell produces testosterone which a $T^{+}$cell needs. 
- $c>d$ : A $T^{P}$ cell will be more fit when interacting with a $T^{+}$cell than compared to its interaction with a $T^{-}$cell, as a $T^{P}$ cell gains extra resources from cellular infrastructure that a $T^{+}$cell builds.

- $e>f:$ A $T^{-}$cell profits more from interacting with a $T^{+}$cell than from interacting with a $T^{P}$ cell, because a $T^{-}$cell may profit from the cellular infrastructure the $T^{+}$ cell produces, while it cannot utilize a systematic testosterone produced by a $T^{P}$ cell.

While we know well which of the coefficients in the fitness matrix $A$ are bigger than others, it is currently impossible to measure their precise values. Therefore, as it was done in Zhang et al. (2017), we assume that parameters $a-f$ have distinct values from the set $\{0.1,0.2,0.3,0.4,0.5,0.6\}$. There are 22 different orderings of such coefficients $a-f$ satisfying the six inequalities (Table 5.2), defining 22 distinct payoff matrices. We show how the nonspatial model varies with respect to different $a-f$ matrix coefficients. We provide a sensitivity analysis of the nonspatial model regarding the matrix coefficients in Section C.2.

\section{C.2 Sensitivity analysis}

This section provides sensitivity analysis on nonspatial metastatic castrate-resistant prostate cancer (mCRPC) model with respect to the individual matrix coefficient $a-f$. The protocol is as follows: For each of the values from the set $\{0.1,0.2,0.3,0.4,0.5,0.6\}$, vary the element of matrix $A$ corresponding to this value by adding and subtracting $0.01,0.02, \ldots, 0.09$, for all 22 matrices from Table 5.2, while keeping all other values the same. Note that the position of the element with a particular value in matrix $A$ may vary. For each of these 18 variations, we report how many matrices belong to each of the groups, i.e., group I (positive), group II (neutral) and group III (negative), and mean and variance of the difference of the ESS frequencies obtained with respect to the base case with original values of the parameter. Positive, neutral and negative refer to therapeutic prognosis based on the frequency of Tcells that are unresponsive to abiraterone. The results are summarized in Figure C.1. For all parameter variations the 3 groups of matrices are maintained, which means that our observations are not sensitive to the magnitude of the matrix elements but rather their rank ordering.

\section{C.3 Tables of transient and saturated equilibria}

This section includes tables C.1-C.3 with detailed information regarding the transient and saturated equilibria for all scenarios introduced in Chapter 5.

\section{C.4 Effects of zero diagonal}

Here, we explore the consequences of having 0's along the diagonal of our matrix model. To do so we added 0.2 to all elements of payoff matrix $A$. We shall refer to this matrix with 


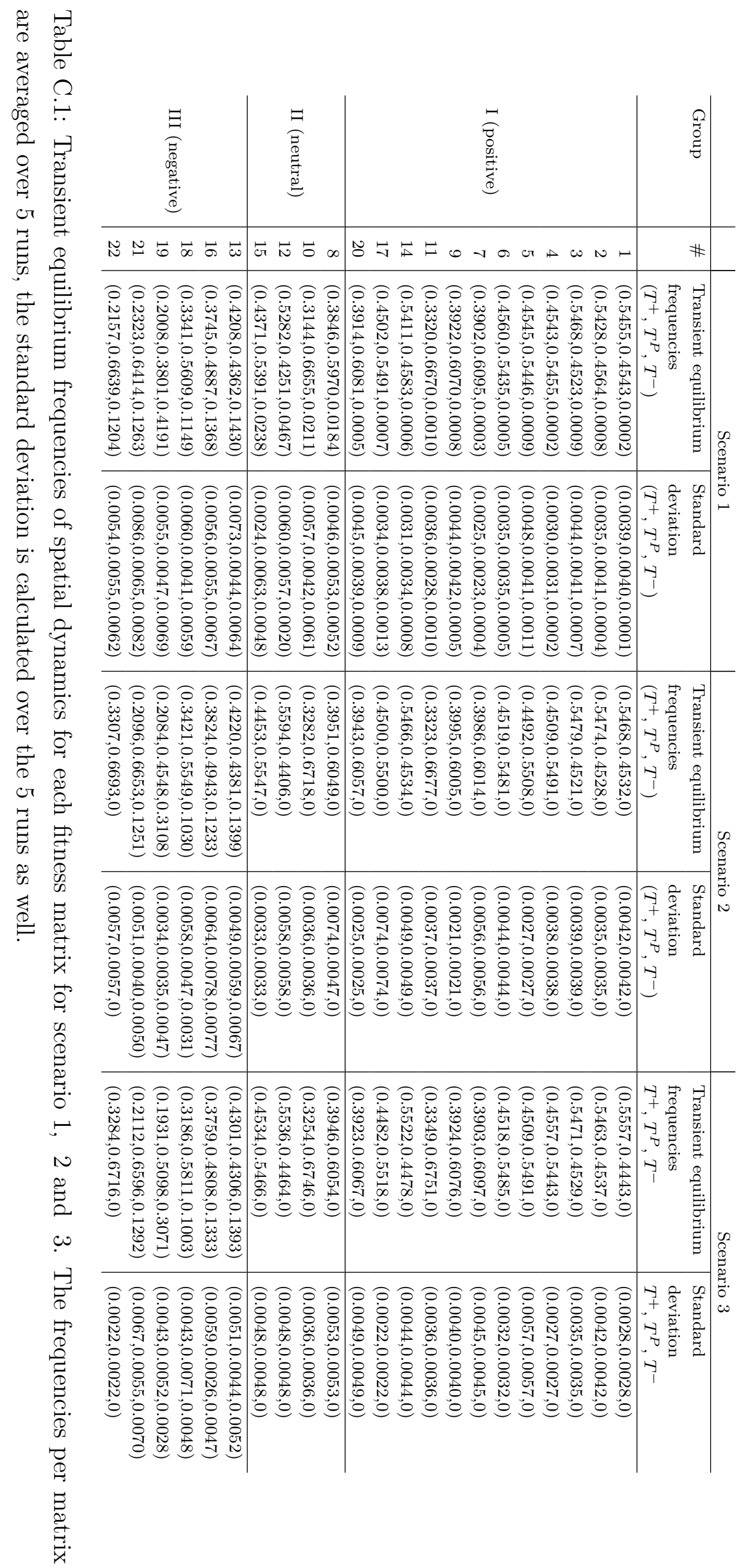




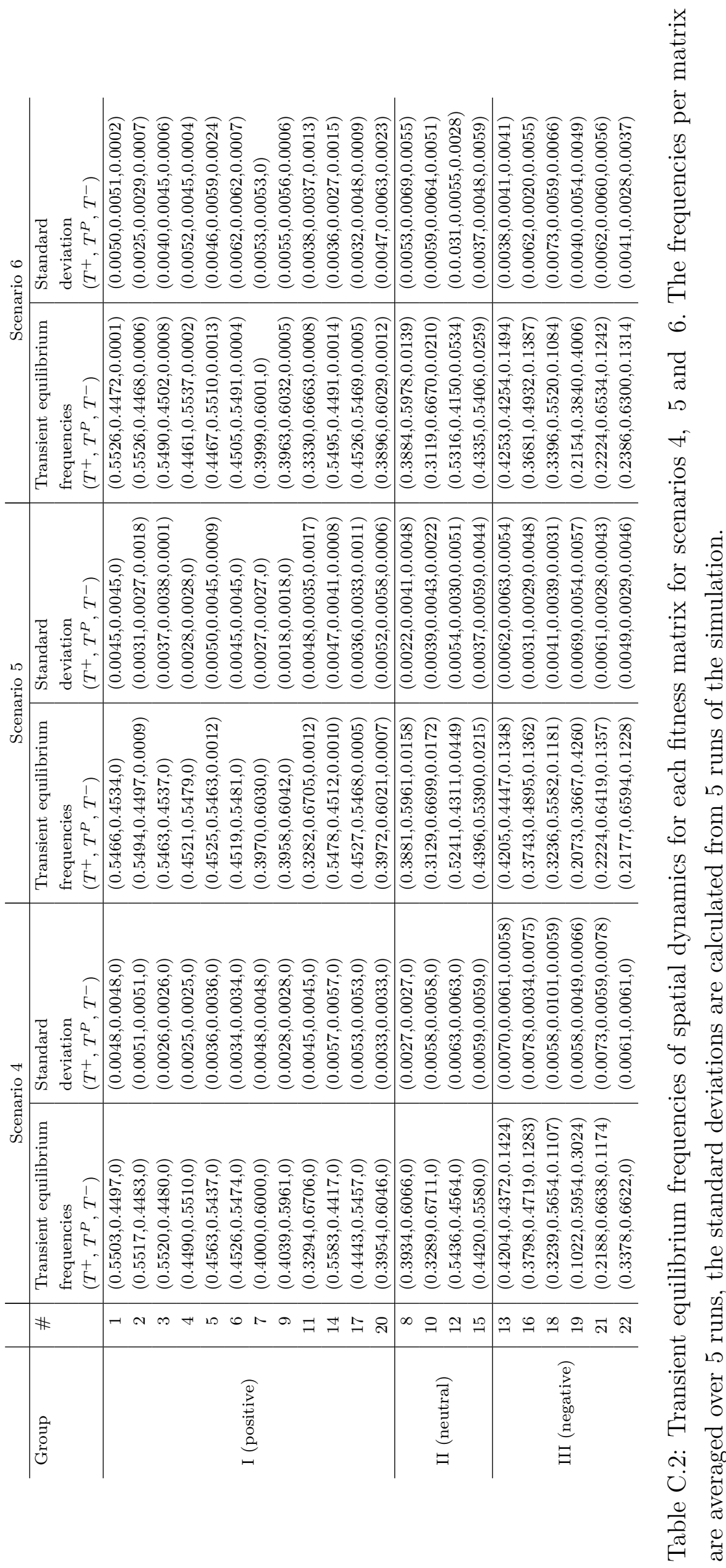




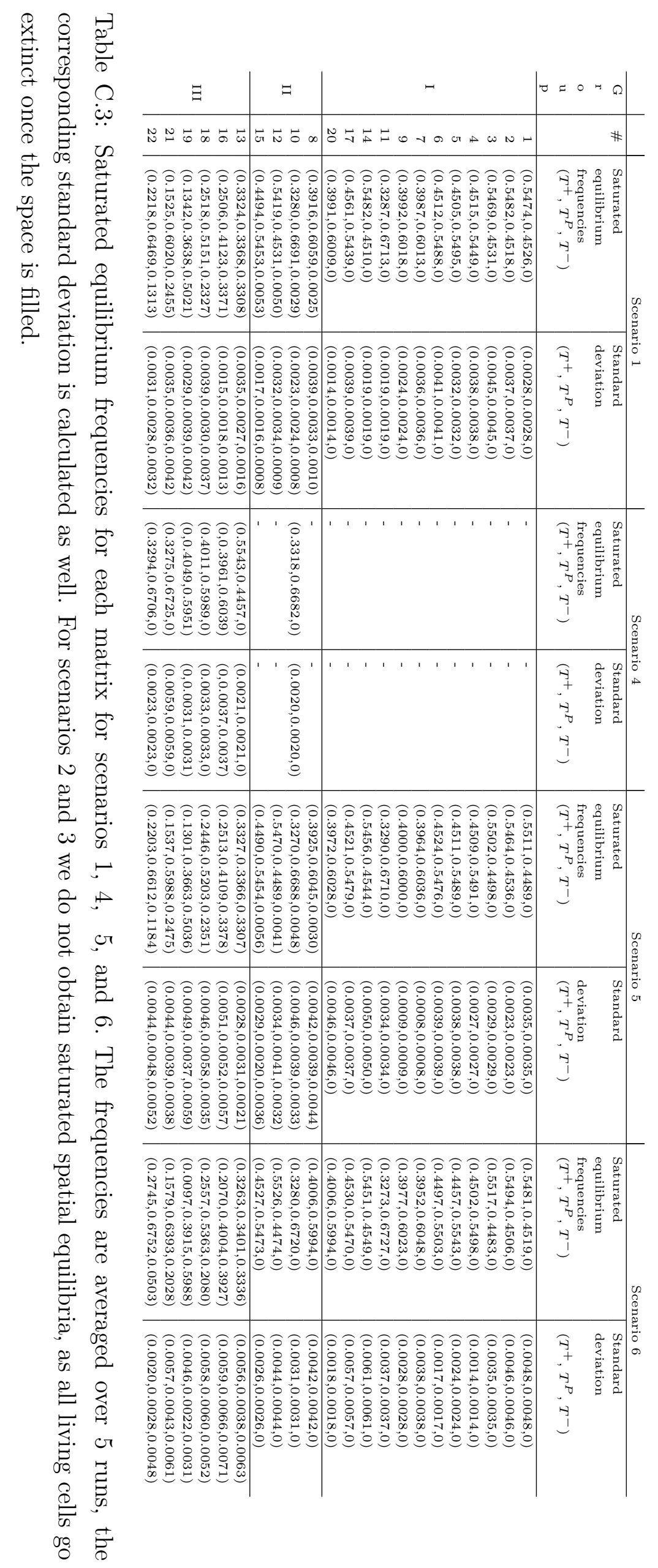




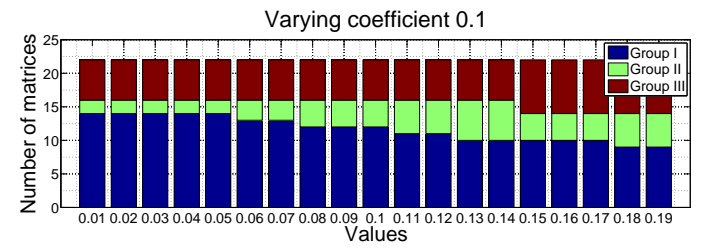

(a) Mean distance is 0.0211 and variance is 0.0012

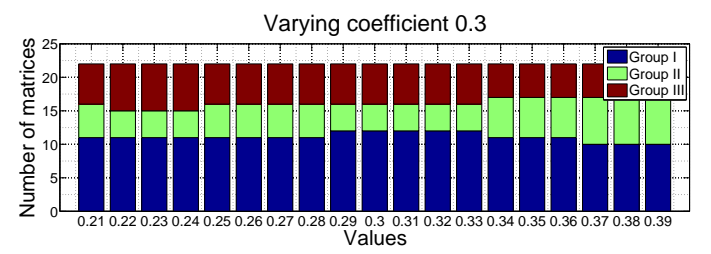

(c) Mean distance is 0.0274 and variance is 0.0018

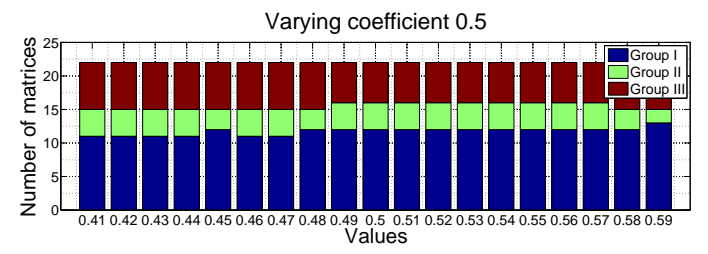

(e) Mean distance is 0.0336 and variance is 0.0009

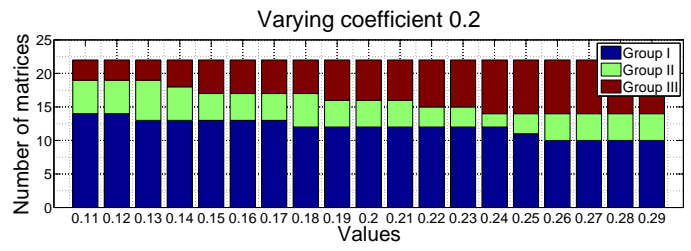

(b) Mean distance is 0.0304 and variance is 0.0023

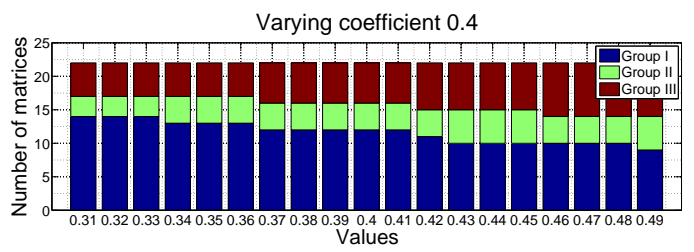

(d) Mean distance is 0.0320 and variance is 0.0016

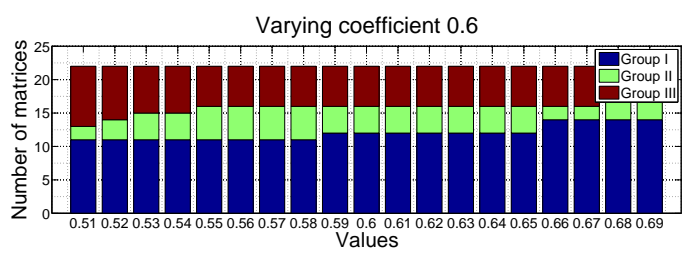

(f) Mean distance is 0.0320 and variance is 0.0005

Figure C.1: Division of the matrices into groups per variation of the value from the set $\{0.1,0.2,0.3,0.4,0.5,0.6\}$

increased elements as the enhanced matrix as opposed to the original matrix. By adding a constant amount to each element, the nonspatial ESSs remain the same (Table 5.2).

We focused on Scenario 1 (mortality regime: $5 \%$ stochastic death rate and removal of dead cells after 5 generations). We examined three representative matrices, enhanced matrices \#7, \#8 and \#22 from groups I, II and III, respectively, under all combinations of the three frequency-dependence radii $(=1,10,50)$, the three density-dependence radii $(=1,10,50)$, and the three dispersal radii $(=1,10,50)$. In total there are 21 matrix and parameter combinations. We ran simulations for 2000 generations and ran five replicates for each parameter combination. The five replicates exhibited very small standard deviations in terms of equilibrium frequencies and variance-to-mean ratios of cell types. The following tables show the average outcome for each enhanced matrix.

In terms of conclusions, there were no qualitative differences between the results from the enhanced and original matrices (Tables C.5, C.9, C.10 and C.11 and Figures C.2 and C.3). When the dispersal, frequency-dependence and density-dependence radii are small, the enhanced matrices produce greater within cell-type clumping (higher variance-to-mean ratios). This is to be expected. When the density-dependence limit permits, a cell that interacts with its own type now has some probability of proliferating which adds another like-type cell to the clump. When the enhanced matrix has no $T^{-}$at the nonspatial ESS, both the enhanced and original matrices for the spatial game result in the ESS frequencies of $T^{+}$and $T^{P}$ cells. When the nonspatial ESS has a very small frequency of $T^{-}$, the resulting spatial game (for enhanced matrix) results in a near absence of $T^{-}$. When the nonspatial 
ESS has a sizable frequency of $T^{-}$, the resulting spatial game (for enhanced matrix) stabilizes on frequencies of $T^{-}$that are below the nonspatial ESS. However, the discrepancy between $T^{-}$in the spatial game (for enhanced matrix) and the nonspatial game becomes smaller.

In exploring the consequences of increasing either the frequency-dependence, dispersal, or density-dependence radii in the spatial games, the games with enhanced matrices yield higher variance-to-mean ratios for the increased frequency-dependence radii (Tables C.6 and C.9), but there is little change when the other 2 radii are increased as well (Tables C.7, C.10, C.8 and C.11). When increasing these particular radii, the resulting spatial equilibrium frequencies of the original and enhanced matrices are almost identical. Visually, there is no difference regarding the dispersion of cells when the density-dependence radius is 10 or 50 , in comparison to the original matrices. For both, a density-dependence radius of 10 still yields numerous small clumps of cells across the space (Figure C.2) and a radius of 50 yields a single large cluster in the middle of the space (Figure C.3).

\begin{tabular}{l|l|l|l}
$\#$ & $\begin{array}{l}\text { ESS } \\
\left(T^{+}, T^{P}, T^{-}\right)\end{array}$ & $\begin{array}{l}\text { Saturated equilibrium frequencies } \\
\left(T^{+}, T^{P}, T^{-}\right)\end{array}$ & $\begin{array}{l}\text { Variance-to-mean ratio } \\
\left(T^{+}, T^{P}, T^{-}\right)\end{array}$ \\
\hline 7 & $(0.4000,0.6000,0)$ & $(0.3979,0.6021,0)$ & $(2.2267,1.5470,-)$ \\
\hline 8 & $(0.3659,0.5659,0.0682)$ & $(0.3752,0.6133,0.0115)$ & $(2.1368,1.3431,10.2605)$ \\
\hline 22 & $(0.2016,0.6285,0.1699)$ & $(0.2214,0.6217,0.1569)$ & $(5.0451,1.4141,6.7156)$
\end{tabular}

Table C.4: The frequencies and variance-to-mean ratios achieved at the saturated spatial equilibrium for the enhanced matrices, when dispersal, frequency-dependence and densitydependence radii are all equal to 1 . The standard deviation of spatial equilibrium frequencies and variance-to-mean ratios over 5 runs are reported in Table C.5. Table 5.4 shows saturated equilibrium frequencies and variance-to-mean ratios of the original matrices.

\begin{tabular}{l|l|l}
$\#$ & $\begin{array}{l}\text { Std of spatial equilibrium frequencies } \\
\left(T^{+}, T^{P}, T^{-}\right)\end{array}$ & $\begin{array}{l}\text { Std of variance-to-mean ratio } \\
\left(T^{+}, T^{P}, T^{-}\right)\end{array}$ \\
\hline 7 & $(0.0031,0.0031,0)$ & $(0.0223,0.0291,0)$ \\
\hline 8 & $(0.0044,0.0037,0.0013)$ & $(0.0300,0.0158,0.0397)$ \\
\hline 22 & $(0.0042,0.0034,0.0029)$ & $(0.0413,0.0207,0.0138)$
\end{tabular}

Table C.5: The standard deviation of spatial equilibrium frequencies and variance-to-mean ratios over 5 runs 


\begin{tabular}{l|l|l|l}
$\#$ & $\begin{array}{l}\text { ESS } \\
\left(T^{+}, T^{P}, T^{-}\right)\end{array}$ & $\begin{array}{l}\text { Saturated equilibrium frequencies } \\
\left(T^{+}, T^{P}, T^{-}\right)\end{array}$ & $\begin{array}{l}\text { Variance-to-mean ratio } \\
\left(T^{+}, T^{P}, T^{-}\right)\end{array}$ \\
\hline 7 & $(0.4000,0.6000,0)$ & $(0.3970,0.6030,0)$ & $(17.9747,15.2977,0)$ \\
\hline 8 & $(0.3659,0.5659,0.0682)$ & $(0.3757,0.5686,0.0557)$ & $(16.0118,11.0791,18.6105)$ \\
\hline 22 & $(0.2016,0.6285,0.1699)$ & $(0.2138,0.6231,0.1631)$ & $(11.0032,7.1923,13.8021)$
\end{tabular}

Table C.6: The frequencies and variance-to-mean ratios achieved at the saturated spatial equilibrium for enhanced matrices, when the dispersal and density-dependence radii are both set to 1 , while the frequency-dependence radius is 10 . The standard deviations of the equilibrium frequencies and variance-to-mean ratios are very small and comparable to those in Table C.5. For comparison, Figure 5.7 shows the saturated equilibrium frequencies and variance-to-mean ratios of the original matrices.

\begin{tabular}{l|l|l|l}
$\#$ & $\begin{array}{l}\text { ESS } \\
\left(T^{+}, T^{P}, T^{-}\right)\end{array}$ & $\begin{array}{l}\text { Saturated equilibrium frequencies } \\
\left(T^{+}, T^{P}, T^{-}\right)\end{array}$ & $\begin{array}{l}\text { Variance-to-mean ratio } \\
\left(T^{+}, T^{P}, T^{-}\right)\end{array}$ \\
\hline 7 & $(0.4000,0.6000,0)$ & $(0.3966,0.6034,0)$ & $(2.6659,3.7850,-)$ \\
\hline 8 & $(0.3659,0.5659,0.0682)$ & $(0.3682,0.5603,0.0715)$ & $(2.5072,3.5029,2.9234)$ \\
\hline 22 & $(0.2016,0.6285,0.1699)$ & $(0.2003,0.6309,0.1688)$ & $(2.1402,3.5098,1.9857)$
\end{tabular}

Table C.7: The frequencies and variance-to-mean ratios achieved at the saturated spatial equilibrium for enhanced matrices, when the frequency-dependence and density-dependence radii are set to 1 , while the dispersal radius is 10 . The standard deviations of the equilibrium frequencies and variance-to-mean ratios are very small and comparable to those in Table C.5. For comparison, Figure 5.8 shows the saturated equilibrium frequencies and variance-to-mean ratios of the original matrices.

\begin{tabular}{l|l|l|l}
$\#$ & $\begin{array}{l}\text { ESS } \\
\left(T^{+}, T^{P}, T^{-}\right)\end{array}$ & $\begin{array}{l}\text { Saturated equilibrium frequencies } \\
\left(T^{+}, T^{P}, T^{-}\right)\end{array}$ & $\begin{array}{l}\text { Variance-to-mean ratio } \\
\left(T^{+}, T^{P}, T^{-}\right)\end{array}$ \\
\hline 7 & $(0.4000,0.6000,0)$ & $(0.3970,0.6030,0)$ & $(59.2311,41.9001,-)$ \\
\hline 8 & $(0.3659,0.5659,0.0682)$ & $(0.3468,0.6131,0.0401)$ & $(40.7464,39.8969,51.0012)$ \\
\hline 22 & $(0.2016,0.6285,0.1699)$ & $(0.1898,0.6902,0.1201)$ & $(20.9789,21.1066,28.6123)$
\end{tabular}

Table C.8: The frequencies and variance-to-mean ratios of types achieved at the spatial equilibrium for enhanced matrices, when frequency-dependence and dispersal radii are all equal to 1 , while density-dependence radius is 10 . The standard deviation of the equilibrium frequencies and variance-to-mean ratios are very small and comparable to those in Table C.5. Also see Figure 5.9 for saturated equilibrium frequencies and variance-to-mean ratios of the original matrices. 


\begin{tabular}{l|l|l|l}
$\#$ & $\begin{array}{l}\text { ESS } \\
\left(T^{+}, T^{P}, T^{-}\right)\end{array}$ & $\begin{array}{l}\text { Saturated equilibrium frequencies } \\
\left(T^{+}, T^{P}, T^{-}\right)\end{array}$ & $\begin{array}{l}\text { Variance-to-mean ratio } \\
\left(T^{+}, T^{P}, T^{-}\right)\end{array}$ \\
\hline 7 & $(0.4000,0.6000,0)$ & $(0.4015,0.5985,0)$ & $(27.3108,21.9151,-)$ \\
\hline 8 & $(0.3659,0.5659,0.0682)$ & $(0.3648,0.5653,0.0700)$ & $(26.7213,24.5489,28.6670)$ \\
\hline 22 & $(0.2016,0.6285,0.1699)$ & $(0.2012,0.6300,0.1688)$ & $(33.3553,13.5125,23.4429)$
\end{tabular}

Table C.9: The frequencies and variance-to-mean ratios of types achieved at the spatial equilibrium for enhanced matrices, when dispersal and density-dependence radii are all equal to 1 , while frequency-dependence radius is 50 . The standard deviation of the equilibrium frequencies and variance-to-mean ratios are very small and comparable to those in Table C.5. Also see Figure 5.7 for saturated equilibrium frequencies and variance-to-mean ratios of the original matrices.

\begin{tabular}{l|l|l|l}
$\#$ & $\begin{array}{l}\text { ESS } \\
\left(T^{+}, T^{P}, T^{-}\right)\end{array}$ & $\begin{array}{l}\text { Saturated equilibrium frequencies } \\
\left(T^{+}, T^{P}, T^{-}\right)\end{array}$ & $\begin{array}{l}\text { Variance-to-mean ratio } \\
\left(T^{+}, T^{P}, T^{-}\right)\end{array}$ \\
\hline 7 & $(0.4000,0.6000,0)$ & $(0.4014,0.5986,0)$ & $(1.0855,1.0912,-)$ \\
\hline 8 & $(0.3659,0.5659,0.0682)$ & $(0.3642,0.5667,0.0691)$ & $(1.1300,1.0619,1.0503)$ \\
\hline 22 & $(0.2016,0.6285,0.1699)$ & $(0.1990,0.6304,0.1706)$ & $(0.9689,1.1189,0.9624)$
\end{tabular}

Table C.10: The frequencies and variance-to-mean ratios of types achieved at the spatial equilibrium for enhanced matrices, when frequency-dependence and density-dependence radii are all equal to 1 , while dispersal radius is 50 . The standard deviation of the equilibrium frequencies and variance-to-mean ratios are very small and comparable to those in Table C.5. Also see Figure 5.8 for saturated equilibrium frequencies and variance-to-mean ratios of the original matrices.

\begin{tabular}{l|l|l|l}
$\#$ & $\begin{array}{l}\text { ESS } \\
\left(T^{+}, T^{P}, T^{-}\right)\end{array}$ & $\begin{array}{l}\text { Saturated equilibrium frequencies } \\
\left(T^{+}, T^{P}, T^{-}\right)\end{array}$ & $\begin{array}{l}\text { Variance-to-mean ratio } \\
\left(T^{+}, T^{P}, T^{-}\right)\end{array}$ \\
\hline 7 & $(0.4000,0.6000,0)$ & $(0.4005,0.5995,0)$ & $(1.1327,1.0842,-)$ \\
\hline 8 & $(0.3659,0.5659,0.0682)$ & $(0.3686,0.5669,0.0644)$ & $(1.0648,0.9689,1.0774)$ \\
\hline 22 & $(0.2016,0.6285,0.1699)$ & $(0.1979,0.6312,0.1709)$ & $(1.0221,1.1004,1.0951)$
\end{tabular}

Table C.11: The frequencies and variance-to-mean ratios of types (only the central zone i.e., sub-square whose number of total cells is above 200, is measured) achieved at the spatial equilibrium, for enhanced matrices, when frequency-dependence and dispersal radii are all equal to 1 , while density-dependence radius is 50 . The standard deviation of the equilibrium frequencies and variance-to-mean ratios are very small and comparable to those in Table C.5. Also see Figure 5.9 for saturated equilibrium frequencies and variance-to-mean ratios of the original matrices. 


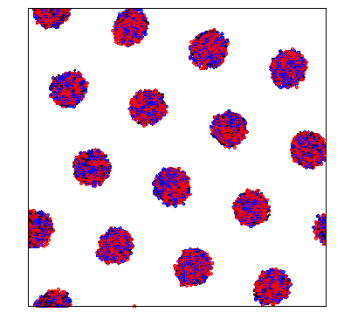

(a)

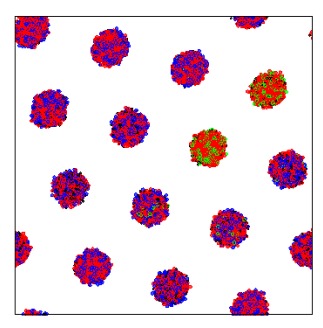

(b)

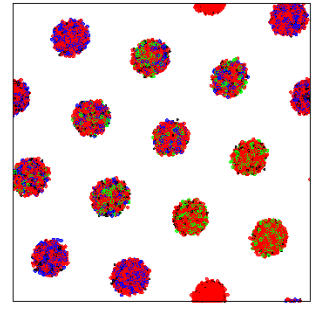

(c)

Figure C.2: The appearance of the simulated tumors for enhanced matrices (a) \#7, (b) \#8 and (c) \#22, when the density-dependence radius is set to 10 while the other two radii are held at 1. Each figure is at saturated equilibrium following 2000 generations.

Frequency-dependence radius $=\mathbf{5 0}$

Dispersal radius $=1$

Density-dependence radius $=1$

Enhanced Matrix \#7

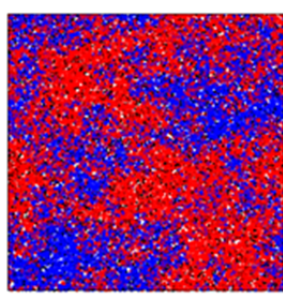

Frequency-dependence radius $=1$

Dispersal radius $=\mathbf{5 0}$

Density-dependence radius $=1$

Frequency-dependence radius $=1$

Dispersal radius $=1$

Density-dependence radius $=\mathbf{5 0}$

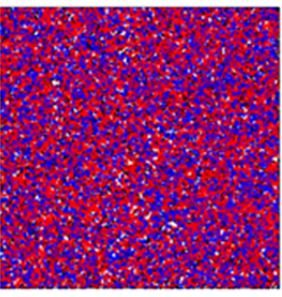

Enhanced Matrix \#8
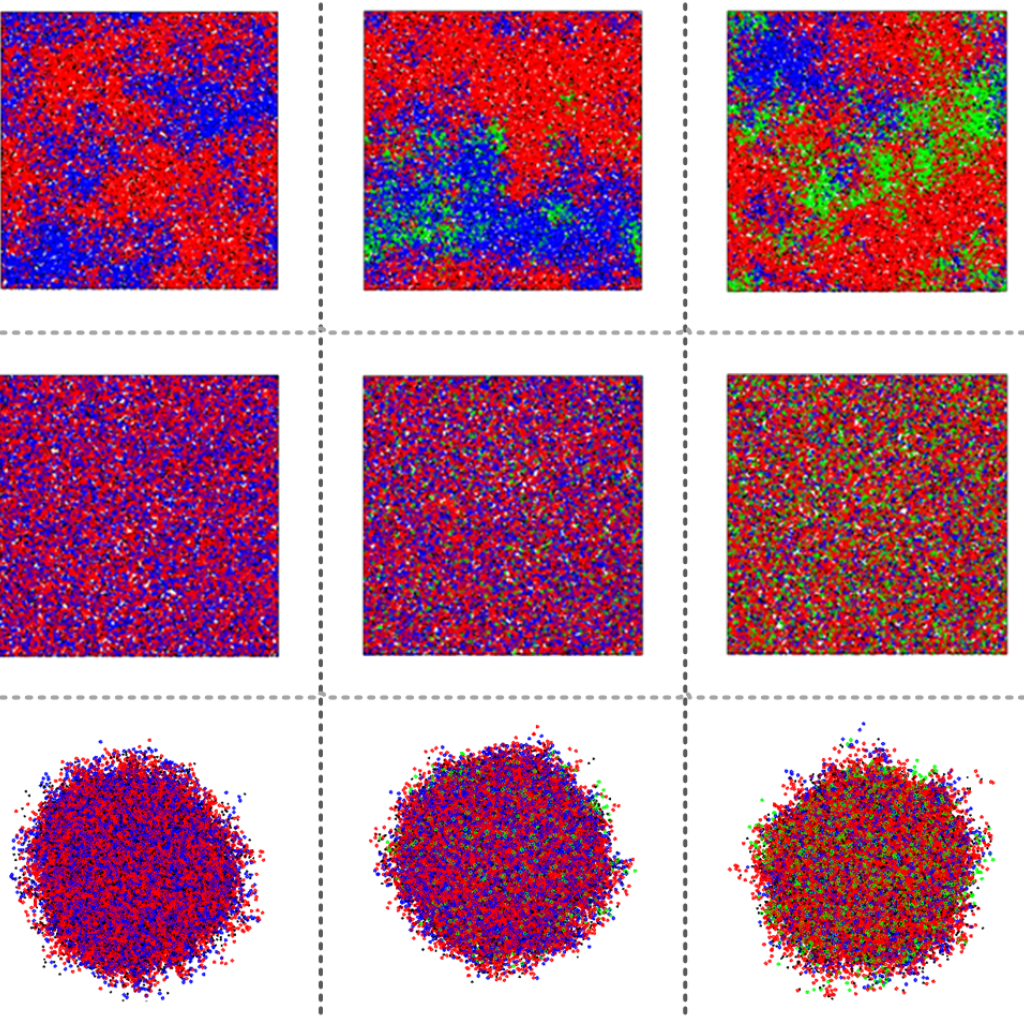

Figure C.3: Simulated tumors for enhanced matrices resulting from setting either the frequency-dependence, dispersal, or density-dependence radius to the highest level (shown as the rows) while holding the other two at 1: The columns represent different enhanced matrices, and the simulations were run for 2000 generations insuring a saturated equilibrium. 


\section{Acknowledgements}

There are a number of people and institutions behind this thesis that deserve to be acknowledged.

First and foremost, I would like to express my sincere appreciation to my supervisors. Informally, my PhD journey started with my very first Skype call with Frank (in March, 2012), who, half a year later, provided me the chance to work on this nice research topic at the the Department of Data Science and Knowledge Engineering (DKE) in Maastricht. As my daily supervisor, Frank has always enlightened me with his immense knowledge of evolutionary game theory. He has given me his continuous support throughout this research, and I will always appreciate the time and effort that he has put in. Next, I would like to thank my co-supervisor Katerina, who has been acting as my daily supervisor, for her enormous help and advice in all the time of research. She has been my role model for a woman in science, which will remain having a profound impact on me, no matter if my future career is in academia or in industry. I also want to thank Ralf for sharing his brilliant ideas with me. Our discussion in the Tribunal will be one of the most interesting seminars in my life. Thank you, Frank, Kateřina and Ralf, for your support, useful feedback and patience.

I would like to thank the collaborators of my research. Outside DKE, Monica from Imperial College London, Joel and Jessica from the Moffitt Cancer Center and Fran from University of Seville; within DKE, Philippe and Katharina are those that I have had a great pleasure of working with. Thank you all for being so kind of sharing your knowledge with me.

I would also like to express my gratitude to the members of the assessment committee. I genuinely appreciate their time for reading my thesis, and giving me the important and critical comments for improving the quality of my thesis.

There are two institutions that I would like to mention here. First, I am very much grateful to the China Scholarship Council (CSC) for providing me with the financial support of this research. Second, I thank DKE which gave me the opportunity to participate in a variety of academic activities. Also, I enjoyed our celebration and day-out activities. 
During the past years I have met lots of nice colleagues and friends. Many thanks go to my officemates Philippe, Bijan and Katharina, with whom I have had nice academic and non-academic talks. They gave me wise suggestions and helped me to evolve in learning. I thank Gijs and Rachel for being reviewers of (parts of) my thesis, and for having drinks together. I also thank Haitham, Firat, Nasser and Chiara for the off-work time which were full of fun. During my secondment in the US, I thank Joel for providing me with interesting as well as enlightening talks. With Peter and Jessica, the one-month work in the US was not only efficient but also full of enjoyment. Thank you for sharing your life stories with me. I want to thank all my Chinese friends who have made my time in Maastricht much greatly enjoyable: Wei, Mengxing, Shuang, Hua, Zhenglong, Wenqing, Wenzhao, Siqi, Jie, Yuan, Mengmeng, Cui, Yu, Tian, Tianxiang, Xiahong and Haiyan. I wish you all the best wherever you are. Further, I would like to mention Tom and Michelle, with whom I have had a lot of funny talks and activities together in the weekend.

My special thank goes to Rick, for his unremitting care and encouragement. Also thank you for introducing me to your lovely family.

My deepest gratitude goes to my loving parents. During my life abroad, they have always been understanding and supportive. Thank you, mom and dad!

Li You

December, 2017

Geleen 


\section{About the author}

Li You was born on July 14, 1988 in Sichuan, China. She graduated from Xihua University, China, with a B.Sc. in Computer Science (2010), and she obtained an M.Sc. in Computer Science and Information Security (2013) from the University of Electronic Science and Technology of China. On the 1st of September 2012, she started her PhD at the Department of Data Science and Knowledge Engineering (DKE), Maastricht University. Since then she has been a member of the group Networks and Strategic Optimization (NSO). Combining her computer science skills with game theoretical knowledge obtained at DKE, Li You has carried out

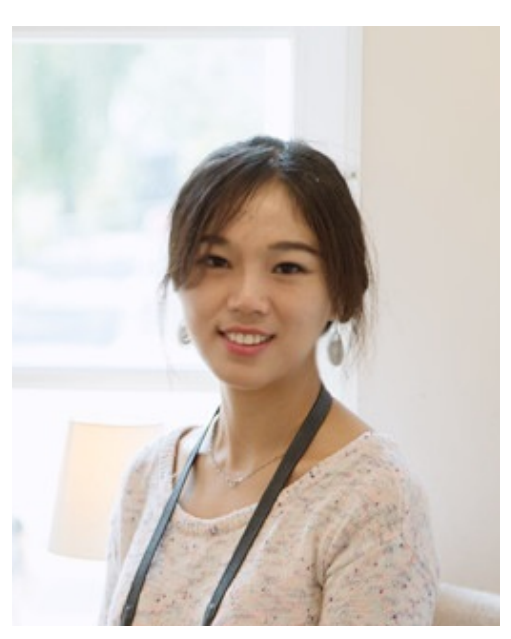
her research focusing on Evolutionary Game Theory and its applications. Her research was jointly founded by the China Scholarship Council, the European Union's Horizon 2020 research and innovation program and the Department of Data Science and Knowledge Engineering, Maastricht University.

During her PhD studies, Li established various international collaborations with researchers from other universities including University of Illinois at Chicago and University of Seville. In 2016, she joined the European Union's Horizon 2020 research and innovation program, and focused on modeling tumorigenesis through a game-theoretical approach. She paid a one-month visit to the University of Illinois at Chicago within this project, and since then she has cooperated with evolutionary biologists and cancer specialists from the University of Illinois at Chicago and from the Moffitt Cancer Center, Tampa, Florida. Her scientific research contributions have led to publications in international journals, books and conference proceedings. 


\section{Publications}

\section{Journal articles}

You Li, Brown Joel. S., Thuijsman Frank, Cunningham Jessica. J., Gatenby Robert. A., Zhang Jingsong, Staňková Kateřina, 2017. Spatial vs. non-spatial eco-evolutionary dynamics in a tumor growth model. Journal of Theoretical Biology, 435: 78-97.

You Li, Thuijsman Frank, Schüller Katharina, Uyttendaele Philippe, Staňková Kateřina. Discrete-space vs. continuous-space games. In preparation.

Staňková Kateřina, Abate Alessandro, Sabelis Maurice W., Buša Ján, You Li, 2013. Joining or opting out of a Lotka-Volterra game between predators and prey: Does the best strategy depend on modeling energy lost and gained? Interface Focus (Theme Issue of the Royal Society Interface), 3(6), paper nr. 20130034.

\section{Book chapter}

Abrudan Monica, You Li, Staňková Kateřina, Thuijsman Frank, 2016. A game theoretical approach to microbial coexistence. In: Thuijsman Frank and Wagener Florian, editors, Advances in Dynamic and Evolutionary Games. Annals of the International Society of Dynamic Games, volume 14, chapter 13, pages 267-282. Springer.

\section{Conference proceedings}

Muros Francisco J., Maestre José M., You Li, Staňková Kateřina, 2017. Model predictive control for optimal treatment in a spatial cancer game. The 56th IEEE Conference on Decision and Control. Accepted.

\section{Presentations}

"Numerical solution of a Stackelberg game between predatory and prey mites", talk at the 16th International Symposium on Dynamic Games and Applications, July 2014, Amsterdam, the Netherlands.

"Modeling prostate cancer as a spatial game", poster presentation at the workshop Game Theory and Evolutionary Biology: Exploring Novel Links, April 2016, Lorentz Center, Leiden, the Netherlands.

"Local evolutionary dynamics in a continuous space", poster presentation at 5th World Congress of the Game Theory Society, July 2016, Maastricht, the Netherlands.

"Modeling tumor growth as a spatial game", talk at the workshop Conflict, Competition, 
Cooperation and Complexity: Using Evolutionary Game Theory to Model Realistic Populations, July 2017, City University, London, United Kingdom.

"Spatial game of metastatic-castrate resistant prostate cancer", talk at the conference Mathematical Models in Ecology and Evolution, July 2017, City University, London, United Kingdom. 